\title{
Strength and Dynamics of Multivalent Complexes at Surfaces
}

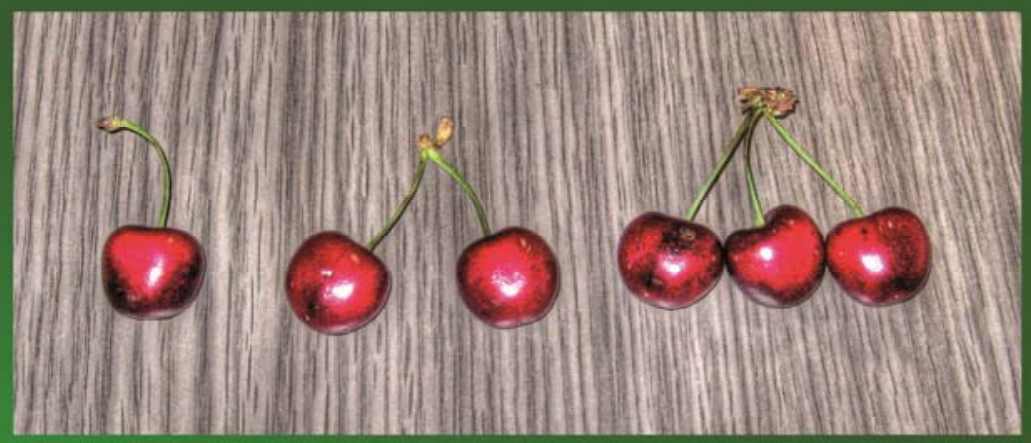

Alberto Gomez-Casado 
Strength and Dynamics of Multivalent Complexes at Surfaces 
The research described in this thesis was performed at the Molecular Nanofabrication group within the MESA+ Institute for Nanotechnology and Department of Science and Technology of the University of Twente. This research was supported by NanoNed, a national nanotechnology program coordinated by the Dutch Ministry of Economic Affairs.

\section{Committee members:}

Chairman: Prof. Dr. G. van der Steenhoven University of Twente Promotor: $\quad$ Prof. Dr. Ir. J. Huskens University of Twente Assistant Promotor: Dr. Ir. P. Jonkheijm University of Twente Members: $\quad$ Prof. Dr. W. J. Briels University of Twente Prof Dr. Ir. D. N. Reinhoudt University of Twente Prof. Dr. Ir. H. Zandvliet University of Twente Prof. Dr. B. J. Ravoo University of Münster Prof. Dr. H. Schönherr University of Siegen

Title: $\quad$ Strength and Dynamics of Multivalent Complexes at Surfaces Author: $\quad$ Alberto Gomez-Casado

ISBN: $\quad 978-90-365-3212-9$

DOI: $\quad 10.3990 / 1.9789036532129$

Copyright (C) 2011 by Alberto Gomez-Casado, Enschede, the Netherlands. All rights reserved. 


\title{
STRENGTH AND DYNAMICS OF MULTIVALENT COMPLEXES AT
} SURFACES

\section{PROEFSCHRIFT}

\author{
ter verkrijging van \\ de graad van doctor aan de Universiteit Twente, \\ op gezag van de rector magnificus, \\ Prof. Dr. H. Brinksma, \\ volgens besluit van het College voor Promoties \\ in het openbaar te verdedigen \\ op vrijdag 15 juli 2011 om 12.45 uur \\ door \\ Alberto Gomez-Casado
}

geboren op 27 oktober 1981

te Salamanca, Spanje 
Dit proefschrift is goedgekeurd door:

Promotor: Prof. Dr. Ir. J. Huskens

Assistent-promotor: Dr. Ir. P. Jonkheijm 
To my family and my girlfriend 



\section{Table of Contents}

\section{Chapter 1}

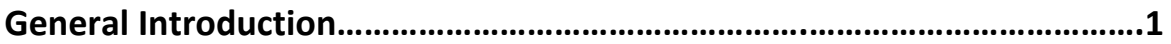

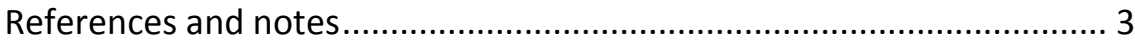

\section{Chapter 2}

Thermodynamics and kinetics of multivalent complexes addressed by dynamic force spectroscopy...............................................................................

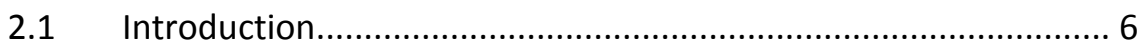

2.2 Theoretical model for multivalent interactions ........................... 9

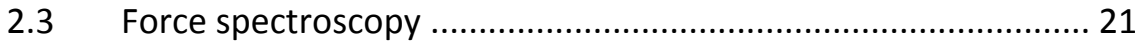

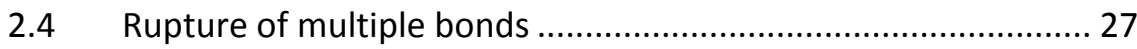

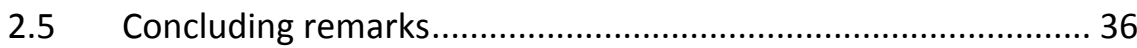

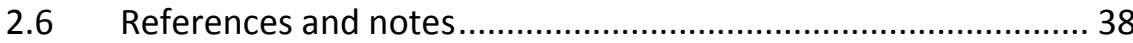

\section{Chapter 3}

Charge-transfer complexes studied by dynamic force spectroscopy........43

3.1 Introduction ....................................................................... 44

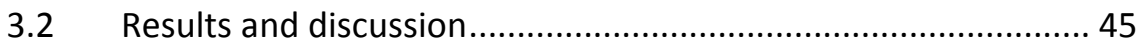

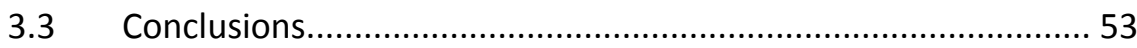

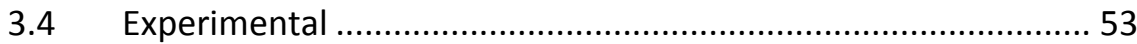

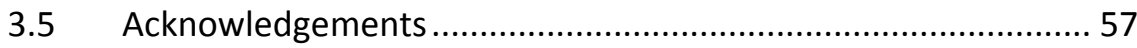

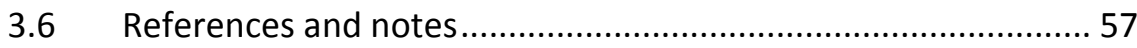

\section{Chapter 4}

Recognition properties of cucurbit[7]uril self-assembled monolayers studied with force spectroscopy

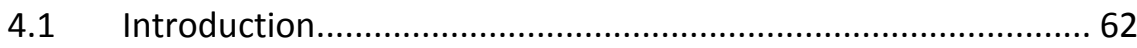

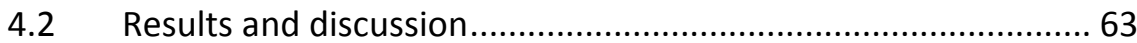

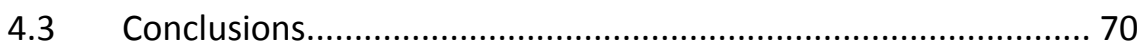




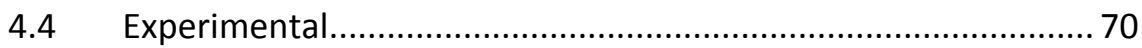

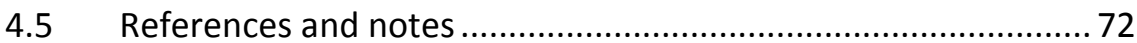

\section{Chapter 5}

Probing multivalent interactions in a synthetic host-guest complex by dynamic force spectroscopy...........................................................................75

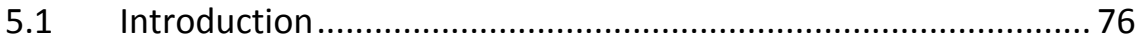

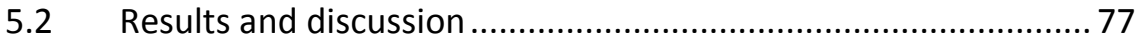

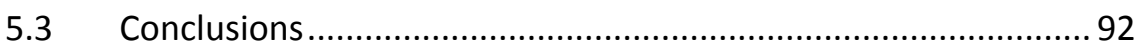

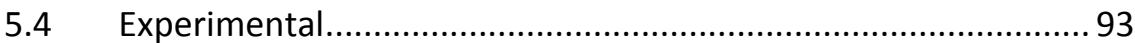

5.5 Appendix A: Thermodynamic equilibrium of monovalent complexes 107

5.6 Appendix B: Effect of the linkers on the stability of the fully-bound

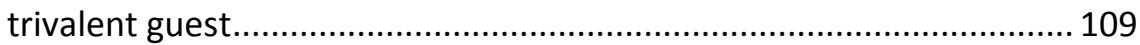

5.7 Acknowledgements ................................................................ 110

$5.8 \quad$ References and notes ............................................................ 111

\section{Chapter 6}

Monte Carlo simulations of the spreading of divalent molecules on a receptor surface .115

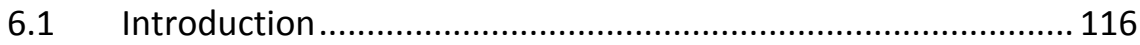

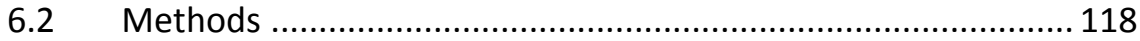

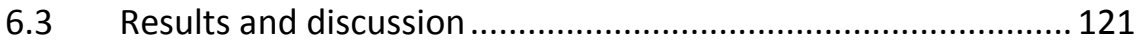

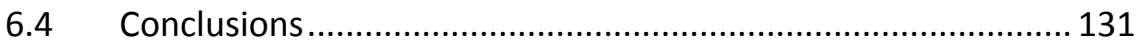

6.5 Appendix A: Mathematical derivations for diffusion constant fitting

6.6 Appendix B: Estimation of spreading through the bulk of solution (flying)

6.7 Appendix C: Estimation of "hop" length versus free host concentration

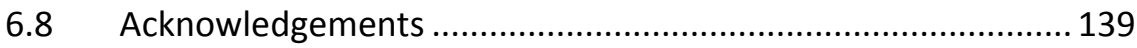

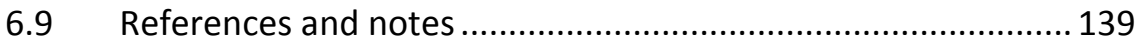


Summary

Sammenvatting .145

List of publications 149

Acknowledgements .151

About the author 157 



\section{Chapter 1}

\section{General introduction}

The purpose of nanotechnology is to control matter at the nanometer level. The fabrication of structures in the 10-100 $\mathrm{nm}$ range enables several applications such as electronics, ${ }^{1}$ optics, ${ }^{2}$ analytics ${ }^{3}$ and medicine. ${ }^{4}$ Two classes of fabrication methods, top-down and bottom-up, have been developed to achieve the required control at the nanoscale. In part to overcome the diffraction limit typically associated with photolithographic tools, and in part to provide cheaper and chemically more versatile processes, alternative lithography techniques have been developed over the past years, such as microcontact printing, ${ }^{5}$ nanoimprint lithography, ${ }^{6}$ e-/X-ray beam lithography and dip-pen nanolithography. ${ }^{7}$ On the other hand, following a bottom-up approach, complex nanostructures can be fabricated from simpler molecular building blocks that self-assemble into the desired conformation. Although these two approaches are entirely different they can be applied in concert. For example, using microcontact printing or dip-pen nanolithography predetermined areas of a surface can be covered with functional molecules. While the positioning of these molecules on the surface results from a topdown strategy, their adhesion, packing and orientation, which often determine the functionality of the structure, result from a self-assembly process.

The spatial distribution of building blocks is, however, not the sole determining factor in controlling function. The evolution in time of the assemblies is also a parameter for the successful design and application of new devices, regardless whether the intended aim is to obtain long-term stability or fast switching. Any dynamic behavior usually originates from the molecular structure of the building blocks and the connections between them. On the other hand, almost every system in Nature is an example of self-assembly and dynamics combined with the reversibility and specificity of supramolecular (non-covalent) bonds in order to build functional structures at the nanometer scale. ${ }^{8}$ Although a single supramolecular bond usually exhibits very fast dissociation kinetics, the stability requirements can be fulfilled by using several of these bonds simultaneously, in a multivalent fashion. ${ }^{9}$ Moreover, the individual dynamics of various processes 
are commonly intertwined in large complex dynamic networks that result in biological function. Therefore, the use of supramolecular chemistry in combination with multivalent strategies appears as a promising design principle in nanotechnology. ${ }^{10}$ Thus, understanding and controlling the dynamics of processes at the nanometer scale is key to fabricating new devices with enhanced stability, response and ultimately function. ${ }^{11}$

The research described in this thesis aims to understand the kinetics of supramolecular complexes and the effects of multivalency on the dynamic behavior of these complexes. Both experimental (dynamic force spectroscopy, DFS) $)^{12}$ and theoretical (Monte Carlo simulations) ${ }^{13}$ methods were employed to this end.

Chapter 2 presents an overview of multivalency and provides a theoretical background on the thermodynamics and kinetics of multivalent assemblies. The effects of multivalency on the stability of a complex are explained as contributions of different types of cooperativity. The use of DFS as a promising technique to characterize this type of bonds and the existing models predicting the rupture behavior of multiple bonds under stress are introduced as well.

In Chapter 3 two different charge-transfer complexes, pyrene-methylviologen and naphthol-methylviologen are measured at the single molecule level using DFS to compare their kinetic behavior.

Chapter 4 shows how DFS can be used to discriminate between specific and non-specific interactions of an adamantyl guest with a self-assembled monolayer of cucurbit[7]uril hosts.

Chapter 5 presents DFS experiments at the single molecule level to test the thermodynamic and kinetic multivalent models discussed in Chapter 2 . Cooperativity contributions are identified in the results from di- and trivalent assemblies of adamantyl guests binding to $\beta$-cyclodextrin molecular printboards.

Finally, in Chapter 6 the diffusion of divalent molecules over a host covered surface is simulated in order to explain the experimental results obtained using divalent adamantyl guests and $\beta$-cyclodextrin-functionalized surfaces. 


\section{References and notes}

1. Martin, C. R.; Baker, L. A., Science 2005, 309, 67-8.

2. (a) Pita, M.; Krämer, M.; Zhou, J.; Poghossian, A.; Schöning, M. J.; Fernández, V. c. M.; Katz, E., ACS Nano 2008, 2, 2160-6; (b) Shipway, A. N.; Katz, E.; Willner, I., ChemPhysChem 2000, 1, 18-52; (c) Kawata, S.; Inouye, Y.; Verma, P., Nat. Photon. 2009, 3, 388-94.

3. Hillie, T.; Hlophe, M., Nat. Nanotechnol. 2007, 2, 663-4.

4. (a) Wagner, V.; Dullaart, A.; Bock, A.-K.; Zweck, A., Nat. Biotech. 2006, 24, 1211-7; (b) Saunders, N. A., Nanomedicine 2011, 6, 271-80; (c) He, J.; Qi, X.; Miao, Y.; Wu, H.-L.; He, N.; Zhu, J.-J., Nanomedicine 2010, 5, 1129-38.

5. (a) Xia, Y.; Whitesides, G. M., Ann. Rev. Mat. Sci. 1998, 28, 153-84; (b) Perl, A.; Reinhoudt, D. N.; Huskens, J., Adv. Mater. 2009, 21, 2257-68.

6. Chou, S. Y.; Krauss, P. R.; Renstrom, P. J., Science 1996, 272, 85-7.

7. (a) Piner, R. D.; Zhu, J.; Xu, F.; Hong, S.; Mirkin, C. A., Science 1999, 283, 661-3; (b) Salaita, K.; Wang, Y.; Mirkin, C. A., Nat. Nanotechnol. 2007, 2, 145-55.

8. (a) Parsons, J. T.; Horwitz, A. R.; Schwartz, M. A., Nat. Rev. Mol. Cell. Biol. 2010, 11, 633-43; (b) Hirokawa, N.; Noda, Y.; Tanaka, Y.; Niwa, S., Nat. Rev. Mol. Cell. Biol. 2009, 10, 682-96; (c) Errington, J., Nat. Cell Biol. 2003, 5, 175-8; (d) Azar, G. A.; Lemaître, F.; Robey, E. A.; Bousso, P., Proc. Natl. Acad. Sci. U.S.A. 2010, 107, 3675-80; (e) Schmidt, B. J.; Papin, J. A.; Lawrence, M. B., PLoS Comput. Biol. 2009, 5, e1000612; (f) Govern, C. C.; Paczosa, M. K.; Chakraborty, A. K.; Huseby, E. S., Proc. Natl. Acad. Sci. U.S.A. 2010, 107, 8724-9.

9. (a) Rankl, C.; Kienberger, F.; Wildling, L.; Wruss, J.; Gruber, H. J.; Blaas, D.; Hinterdorfer, P., Proc. Natl. Acad. Sci. U.S.A. 2008, 105, 17778-83;

(b) Wu, Y.; Jin, X.; Harrison, O.; Shapiro, L.; Honig, B. H.; Ben-Shaul, A., Proc. Natl. Acad. Sci. U.S.A. 2010, 107, 17592-7; (c) Arranz-Plaza, E.; Tracy, A. S.; Siriwardena, A.; Pierce, J. M.; Boons, G. J., J. Am. Chem. Soc. 2002, 124, 13035-46; (d) Mammen, M.; Choi, S.-K.; Whitesides, G. M., Angew. Chem. Int. Ed. 1998, 37, 2754-94; (e) Santoro, S. A.; Cunningham, L. W., J. Clin. Invest. 1977, 60, 1054-60; (f) Pantoliano, M. W.; Horlick, R. A.; Springer, B. A.; Van Dyk, D. E.; Tobery, T.; Wetmore, D. R.; Lear, J. D.; Nahapetian, A. T.; Bradley, J. D.; Sisk, W. P., Biochemistry 1994, 33, 10229-48; (g) Sulzer, B.; Perelson, A. S., Math. Biosci. 1996, 135, 147-85; (h) Kiessling, L. L.; Lamanna, A. C., Multivalency in Biological Systems. In Chemical Probes in Biology, Schneider, M. P., Ed. Kluwer Academic Publishers: Dordrecht, 2003; (i) Sriram, S. M.; Banerjee, R.; Kane, R. S.; Kwon, Y. T., Chem. Biol. 2009, $16,121-31$. 
10. (a) Mulder, A.; Huskens, J.; Reinhoudt, D. N., Org. Biomol. Chem. 2004, 2, 3409-24; (b) Badjic, J. D.; Nelson, A.; Cantrill, S. J.; Turnbull, W. B.; Stoddart, J. F., Acc. Chem. Res. 2005, 38, 723-32.

11. (a) Ling, X. Y.; Phang, I. Y.; Schönherr, H.; Reinhoudt, D. N.; Vancso, G. J.; Huskens, J., Small 2009, 5, 1428-35; (b) Banerjee, D.; Liu, A. P.; Voss, N. R.; Schmid, S. L.; Finn, M. G., ChemBioChem 2010, 11, 1273-9; (c) Vico, R. V.; Voskuhl, J.; Ravoo, B. J., Langmuir 2010, 27, 1391-7; (d) Perumal, S.; Hofmann, A.; Scholz, N.; Rühl, E.; Graf, C., Langmuir 2011, DOl: 10.1021/la105134m; (e) Wickham, S. F. J.; Endo, M.; Katsuda, Y.; Hidaka, K.; Bath, J.; Sugiyama, H.; Turberfield, A. J., Nat. Nanotechnol. 2011, 6, 166-9; (f) Liao, X.; Petty, R. T.; Mrksich, M., Angew. Chem. Int. Ed. 2010, 123, 732-4.

12. (a) Evans, E.; Ritchie, K., Biophys. J. 1997, 72, 1541-55; (b) Neuman, K. C.; Nagy, A., Nat. Methods 2008, 5, 491-505; (c) Hugel, T.; Seitz, M., Macromol. Rapid Commun. 2001, 22, 989-1016.

13. (a) Gillespie, D. T., J. Phys. Chem. 1977, 81, 2340-61; (b) Bernstein, D., Phys. Rev. E Stat. Nonlin. Soft Matter Phys. 2005, 71. 


\section{Chapter 2}

\section{Thermodynamics and kinetics of multivalent complexes addressed by dynamic force spectroscopy}

In this chapter an overview of the concepts relevant for the subject of this thesis is presented. The definition and theoretical understanding of the stability of multivalent complexes is introduced, followed by a review of the most important aspects of force spectroscopy. Finally, the potential of this technique for the study of multivalency is discussed. 


\subsection{Introduction}

Multivalent complexes occur when two molecules are connected via more than one simultaneous interaction. They are the key to many biological interactions and self-assembly strategies. ${ }^{1}$ The combination of several supramolecular interactions yields stable and robust bonds while retaining interesting characteristics of supramolecular complexes such as reversibility and dynamic behavior. The thermodynamic behavior of multivalent interactions is well described, ${ }^{2}$ however the kinetics of these complexes is more challenging to address in a quantitative manner. Force spectroscopy appears to be a technique able to explore the stability of complexes and to evaluate the multivalent character of the interaction.

\subsubsection{Multivalency, definition and importance}

The valency of a molecule is the number of groups present in its molecular structure which are potentially able to bind to a complementary group. In the case of an interaction its valency is the number of simultaneous individual connections between the two or more interacting molecules (see Figure 2.1). Thus, an interaction between two complementary trivalent molecules is not necessarily trivalent as well, the actual valency of the interaction will depend on the orientation of the complementary groups and other factors such as steric hindrance. For the common case of two molecules each is referred to as host and guest or ligand and receptor. 


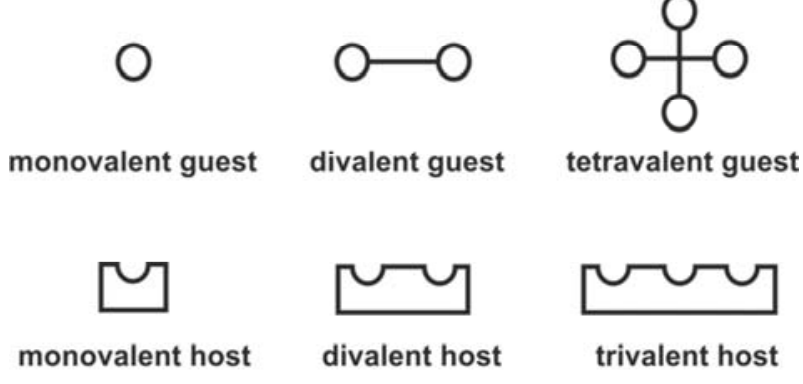

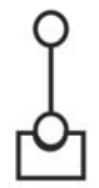

monovalent complex

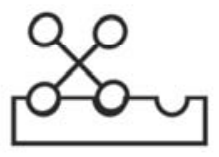

divalent complex

Figure 2.1 Nomenclature of valencies.

\subsubsection{Multivalency in nature}

Multivalent binding is observed very often in natural systems, and plays a fundamental role in several intra- and intercellular processes. ${ }^{10,3}$ The reason for this prevalence of supramolecular multivalent bonds in nature are the stability, reversibility and specificity that can be achieved through this type of interaction. Regarding the stability aspect, covalent bonds are in general much more stable than supramolecular bonds. However, despite recent progress in using covalent bonds for systems chemistry, ${ }^{4}$ covalent links are in general irreversible and thus impractical for dynamic processes. Cells in a tissue need to keep strong connections among them, as well as to the extracellular matrix, but at the same time they must be able to remove and reform these connections during growing and healing processes. Multivalent bonds fulfill both requirements, which explains their presence in many protein-protein interactions and almost any cell-to-cell and cell-to-matrix connections. For example, in adherens junctions several molecules of the transmembrane protein cadherin intercalate to form a very strong adhesion patch between the membranes of two cells. ${ }^{5}$ This keeps the cells together, although the basic interaction between two cadherins is relatively weak and can be modulated by the presence of calcium ions. ${ }^{6}$ Non-covalent interactions such as carbohydratecarbohydrate and carbohydrate-protein interactions can be very specific and 
mediate recognition processes, immune response and cell differentiation. ${ }^{7}$ These interactions are typically too weak to be effective when binding only monovalently, however multivalency can enhance the affinity of the overall interaction. ${ }^{8}$ One example of this is the human $A B O$ blood group system. Each individual expresses some antibodies (anti-A, anti-B) in the serum and a carbohydrate chain linked to the membrane of the red blood cells. Transfusing blood of A or B type into an individual expressing anti-A or anti-B will result in an adverse hemolytic reaction. The only difference between the group carbohydrates is the absence (group $\mathrm{O}$ ) or presence of either one $\mathrm{N}$ acetylgalactosamine (group A) or one galactose (group B). Removal of this antigens from red cells is being explored in order to obtain so-called universal blood. ${ }^{9}$ The recognition is enhanced by the display of many carbohydrates in the cell membrane as well as the fact that anti-A/B antibodies are usually IgM, a pentameric antibody presenting 10 binding sites. This multiplicity of sites is a general feature of all antibodies (IgD, IgE, IgG are divalent, IgA tetravalent and IgM decavalent), and allows effective binding at nanomolar concentrations. Often several binding sites of the antibodies remain free and exposed to the medium, inducing the formation of pathogen aggregates that can be fagocited.

Multivalency appears also in many signal transduction mechanisms. Some membrane receptors, such as protein kinases, can be held in close proximity to other transmembrane proteins by a multivalent ligand, promoting the formation of clusters and the phosphorylation of cytosolic fragments of substrate proteins, providing a way to propagate the signal through the cell membrane. $^{10}$

\subsubsection{Multivalency in nanotechnology}

There is a considerable interest in the application of multivalent strategies in (bio)nanotechnology. ${ }^{1 \mathrm{~b}, \mathrm{c}}$ Antigen binding fragments (Fab areas of antibodies) can be used to target specifically viruses, bacteria or cells. However, monovalent binding is often too weak for a successful application of such fragments. The design of artificial multivalent antibodies appears to be a promising strategy to address this problem and has been explored in the context of cancer therapy, ${ }^{11}$ and the study of signal transduction. ${ }^{12}$ 


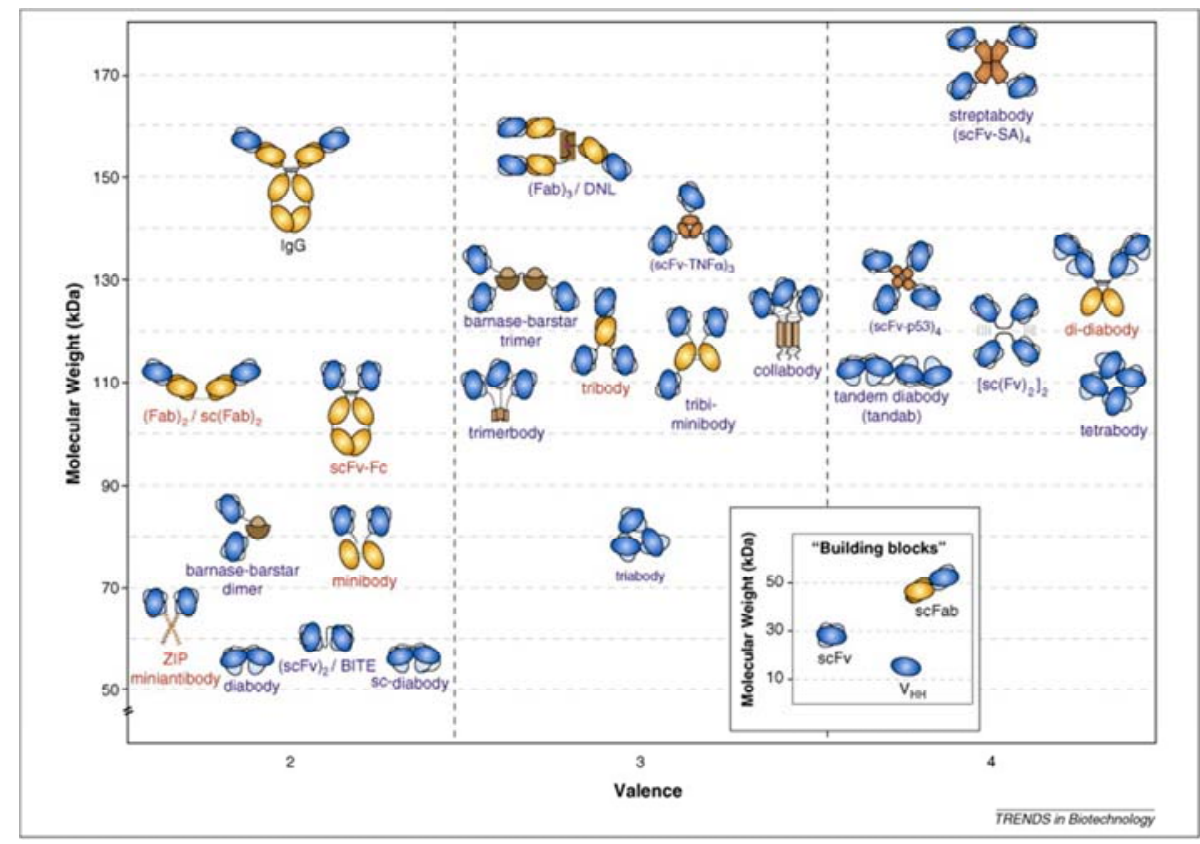

Figure 2.2 Designed multivalent antibodies. (from Cuesta et al, 2010, Reproduced with permission from Elsevier)

Multivalency has been also applied to immobilize nanotubes ${ }^{13}$ and proteins ${ }^{14}$ on a substrate and release them upon applying a stimulus such as light. Furthermore, aggregation of vesicles displaying multiple receptors can be induced by multivalent ligands. ${ }^{15}$ Multivalent dendrimeric connecting units have been used to crosslink virus-like particles ${ }^{16}$ and host-functionalized nanoparticles, which in the latter case could be used to construct macroscopically robust three dimensional structures. ${ }^{17}$

\subsection{Theoretical model for multivalent interactions}

\subsubsection{Cooperativity}

In the past years, several models have been proposed to interpret thermodynamic data obtained from multivalent systems. One of the key points of these models is deciding whether (allosteric) cooperativity is present or not in the binding process. In other words, if the enhanced stability of a particular multivalent complex originates from a change of the intrinsic binding properties 
or just arises from the increased number of interactions between the complementary molecules. An interaction is considered cooperative if the binding of a ligand to one receptor site affects the binding of a second ligand to a neighboring receptor. The classical example of this effect is found in the binding of oxygen molecules by hemoglobin. ${ }^{18}$ After the first oxygen molecule is bound, the full protein structure (a tetramer in the human case) undergoes a conformational change that makes successive bindings more favorable. This effect is called allosterism. In general, if the second binding site can be modified by the first binding event in its conformation, charge distribution or any property relevant for the second host-guest interaction, the process will be cooperative. If this change leads to a decreased binding affinity for the second guest the cooperativity is negative, and if the case of increased binding affinity the cooperativity is positive.

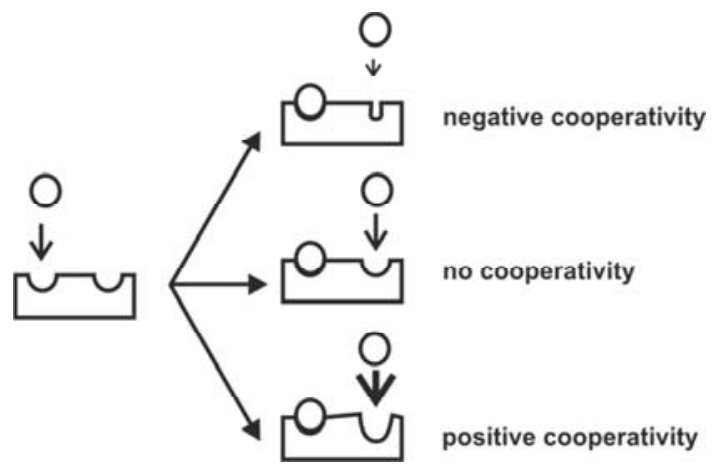

Figure 2.3 Cooperativity in the classical sense (allosterism).

\subsubsection{Inter- and intramolecular binding}

Although at first sight the concept of cooperativity presented above seems clear, in the context of multivalent interactions the precise definition of cooperativity is still a matter of debate. There are two distinct types of binding in the formation of a multivalent complex. The binding of the first interacting pair is always intermolecular and can be fully understood by studying an equivalent monovalent complex. However, successive bindings can be intramolecular, between the already bound pair, or intermolecular, involving other surrounding molecules thus often leading to large aggregates. These two ways of interaction can be prevented or favored depending on the binding sites orientation and steric barriers imposed by the first interaction. 


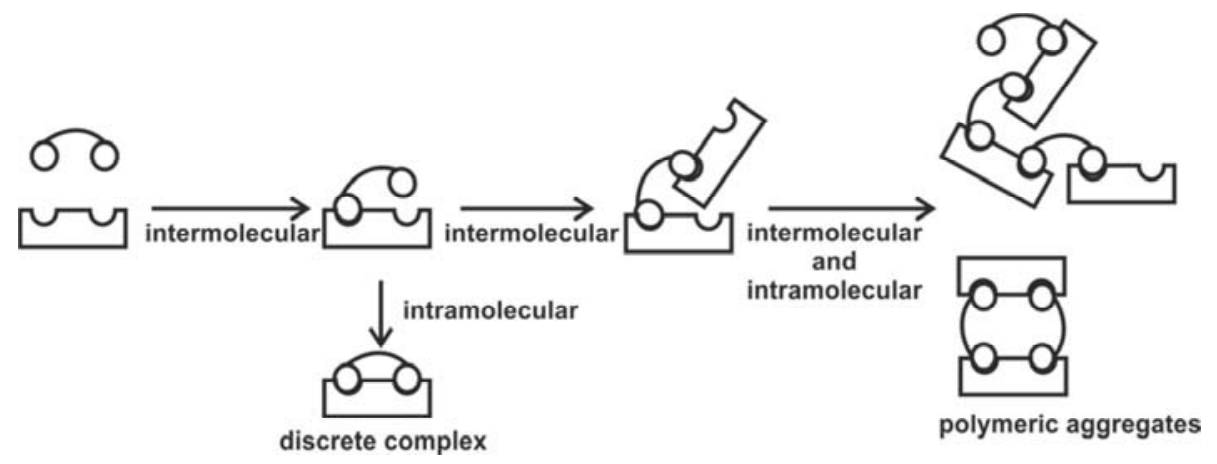

Figure 2.4 Inter and intramolecular binding.

When intramolecular binding occurs, the second and successive binding events may display apparent changes of affinity while the intrinsic host-guest interaction remains unaltered. Let's consider the non-cooperative divalent host of the previous example, and its interaction with a set of divalent guests consisting of identical monovalent guests connected by linkers of different nature. The first binding is similar to the monovalent guest case. Although the interaction between the binding site and the guest moieties is identical regardless of the occupation of the other site, the affinity for the second binding is altered by several factors with opposing effects. On the one hand, the guest is pre-arranged in the vicinity of the binding site, which increases the affinity since part of the loss of entropy associated to the binding process has already been paid. On the other hand, the linker has to adopt a conformation to allow binding of the second guest, which in our example imposes a free energy contribution (in the example elastic potential energy but this could be as well attributed to an entropy loss in the case of polymeric linkers), which lowers the affinity. The final value of the affinity for the second binding will be determined by the interplay between these effects. This has led to claims of negative and positive cooperativity in systems where the intrinsic interaction between each host-guest pair is not expected to change. ${ }^{19}$ 

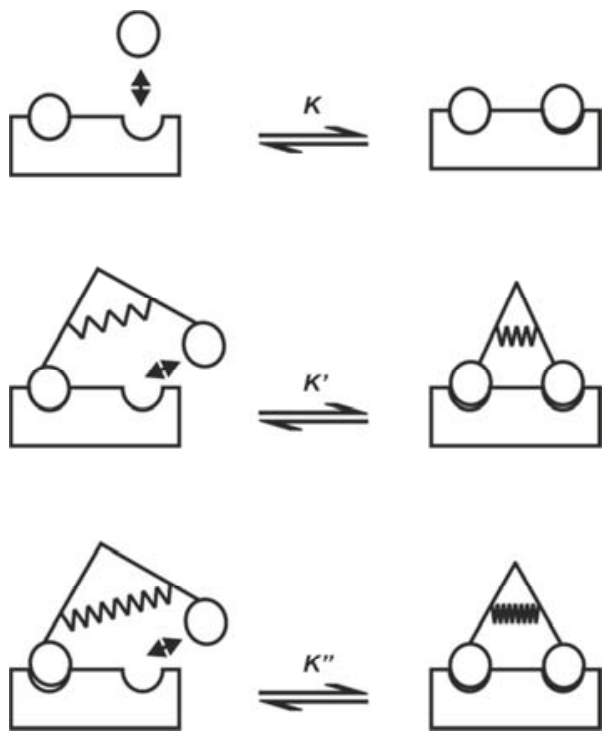

Figure 2.5 Apparent cooperativity with intramolecular binding. Although all the intrinsic interactions are identical, the prearrangement of the second guest in the vicinity of the binding pocket and the hindrance imposed by the molecular structure connecting the moieties modify the affinity of the second binding.

\subsubsection{Cooperativity in multivalent complexes}

In a very recent essay Ercolani and Schiaffino have defined three types of cooperativity; i.e. allosteric, chelate and interannular cooperativity; which can be present alone or combined in a multivalent assembly. ${ }^{20}$ The first one, allosteric cooperativity, implies an alteration of the intrinsic binding properties for the second and/or successive bonds (the cause of cooperativity defined in a classical way). The second case, chelate cooperativity, accounts for the effect of the molecular structure of the complex in the intramolecular binding. And finally, the third, interannullar cooperativity, can only be present when more than one intramolecular binding occurs, the first preorganizing the binding sites in a way that enhances or prevents posterior bindings.

These three types of interaction or a combination of them can be used to describe any multivalent interaction. The equilibrium constant of a multivalent assembly can be predicted by the equation:

$K_{m}=\alpha \gamma K_{\sigma} K^{b} E M^{c}$ 
The factors $\alpha$ and $\gamma$ describe the allosteric and interannular cooperativity of the interaction, respectively. A value different than unity for any of these parameters indicates negative (below one) or positive (over one) cooperativity of the corresponding type. The parameter $\mathrm{K}_{\sigma}$ is an statistical factor modeling the possible permutations of the building blocks that lead to equivalent assemblies. The number of binding interactions in the complex, $b$, and the number of building blocks that conform it, i, determine the degree of cyclicity of the assembly $c=b-i+1$. A non-zero value of $c$ determines the appearance of chelate cooperativity. Finally, $\mathrm{K}$ is the intrinsic equilibrium constant of the monovalent complex and EM is the effective molarity. Note that the equilibrium constant of the multivalent assembly is proportional to $\mathrm{K}^{\mathrm{b}}$.

The values of the factors determining the cooperativity character of a particular system can be obtained from experimental thermodynamic data, as it will be briefly discussed in the next sections.

\subsubsection{Allosteric cooperativity}

The allosteric cooperativity can be evaluated from the microscopic binding constants for the first and second binding events, $K_{1}$ and $K_{2}$, of a monovalent guest to the divalent host compared to the intrinsic binding constant, $K$. Thus, $\alpha$ is defined as $\alpha=K_{1} K_{2} / K^{2}$. Note that to compare the observed equilibrium populations of the different species with the constants $K_{1}$ and $K_{2}$ the statistical factors 2 and $1 / 2$ must be introduced.

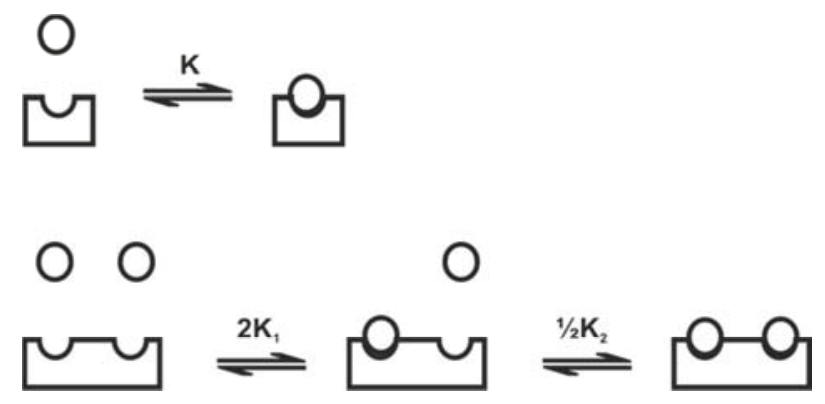

Figure 2.6 Equilibrium constants required to evaluate the allosteric character of a divalent host. 


\subsubsection{Chelate cooperativity, effective molarity and effective concentration}

Chelate cooperativity can only appear when intramolecular binding occurs. The simple case of a divalent host and divalent guest without allosteric cooperativity can be studied as an example. The guest is supposed to be in large excess to the host.

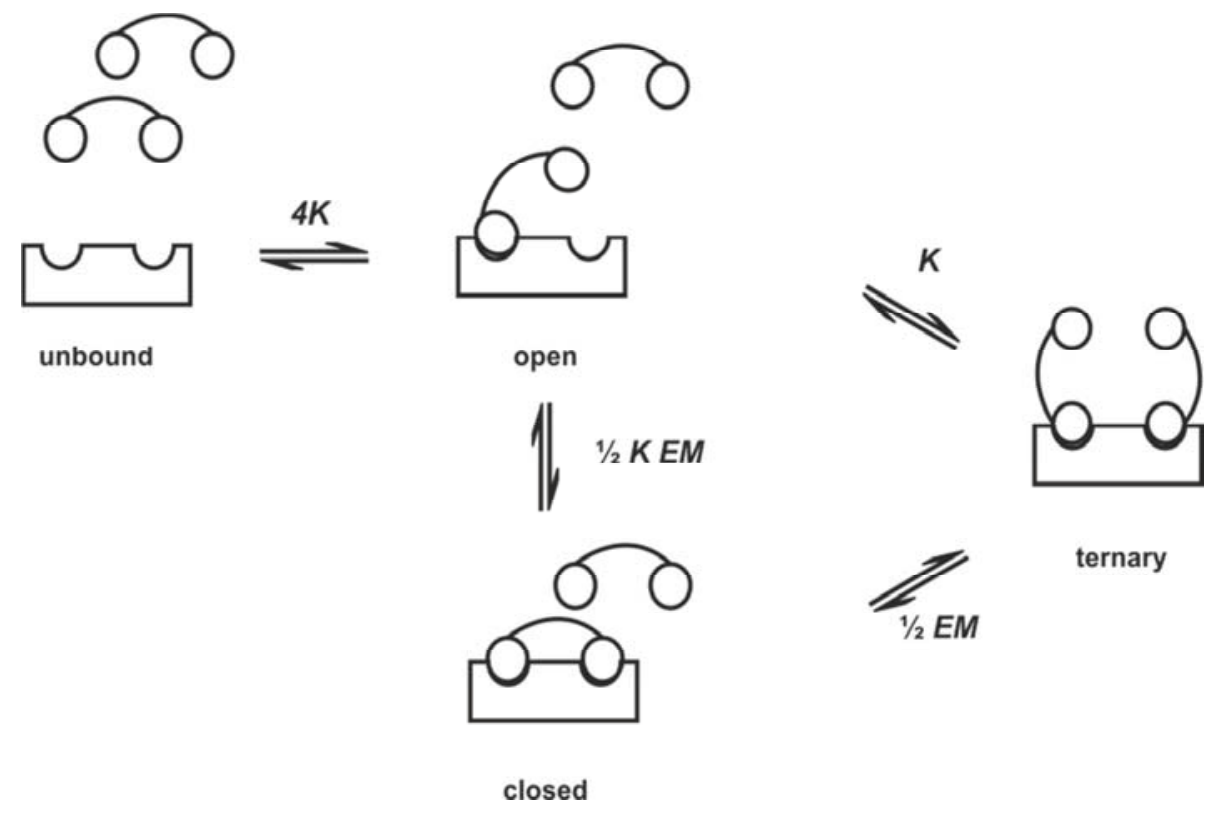

Figure 2.7 Divalent binding equilibria in absence of allosteric cooperativity and excess of guest.

The effective molarity, $E M$, can be obtained studying the speciation of this system at different concentrations of divalent guest. The concentration of divalent guest at which the concentrations of closed and ternary complexes are equal will be $E M / 2$. Concentrations of guest lower than this value will lead to the formation of closed complexes, whereas higher concentrations will lead to the formation of ternary complexes. It is noteworthy that the occurrence of intramolecular binding (and the associated chelate cooperativity) will depend on the concentration of the guest. The value of EM alone cannot be used to assess the presence of chelate cooperativity, it needs to be compared to the molarity of guest. Thus, the factor $\beta=\frac{E M}{2[G G]}$ offers the appropriate measure of 
this type of cooperativity in a system. Deviations from unity will indicate positive $(\beta>1)$ or negative $(\beta<1)$ chelate cooperativity.

Another popular concept characterizing intramolecular binding is the effective concentration, $C_{\text {eff. }}$ It represents the probability that two complementary moieties physically connected to the same assembly will be close enough to bind. ${ }^{21}$ The molecular structure of the multivalent complex is the main factor here, ${ }^{22}$ determining the typical distance between the interacting moieties and the enthalpic and entropic cost of creating a new bond. ${ }^{2 c}$ For example, in the case of a random coil linker the effective concentration will be given by the following expression, in which $r$ is the root-mean-square end-to-end distance, $d$ the distance between binding sites, $N_{A}$ Avogadro's number and $p$ the fraction of the sphere that the second moiety can probe (the presence of the host makes this parameter smaller than unity). ${ }^{23}$

$C_{e f f}=\frac{p\left(\frac{3}{2 \pi}\right)^{\frac{3}{2}}}{N_{A} r^{3}} \exp \left(-\frac{3}{2}\left(\frac{d}{r}\right)^{2}\right)$

A plot based on this expression can be seen in Figure 2.8. Increasing the length of the linker will change the value of the effective concentration from close to zero (no binding sites in the accessible volume), to a maximum value (optimum length of the linker) and then decrease (bigger accessible volume with a fixed number of binding sites).

The effective concentration results from theoretical considerations, thus allows to predict the chelate cooperativity a priori. On the other hand EM results from empirical observations. These two values should be similar for a system where no allosteric cooperativity is present. ${ }^{1 b, 24}$ Furthermore, they offer an estimate for the concentration of competing monovalent guest required to disrupt the multivalent assembly. However, sometimes it is not possible to create solutions of guest at concentrations similar to $E M$ or $C_{\text {eff }}$ because of solubility problems. 

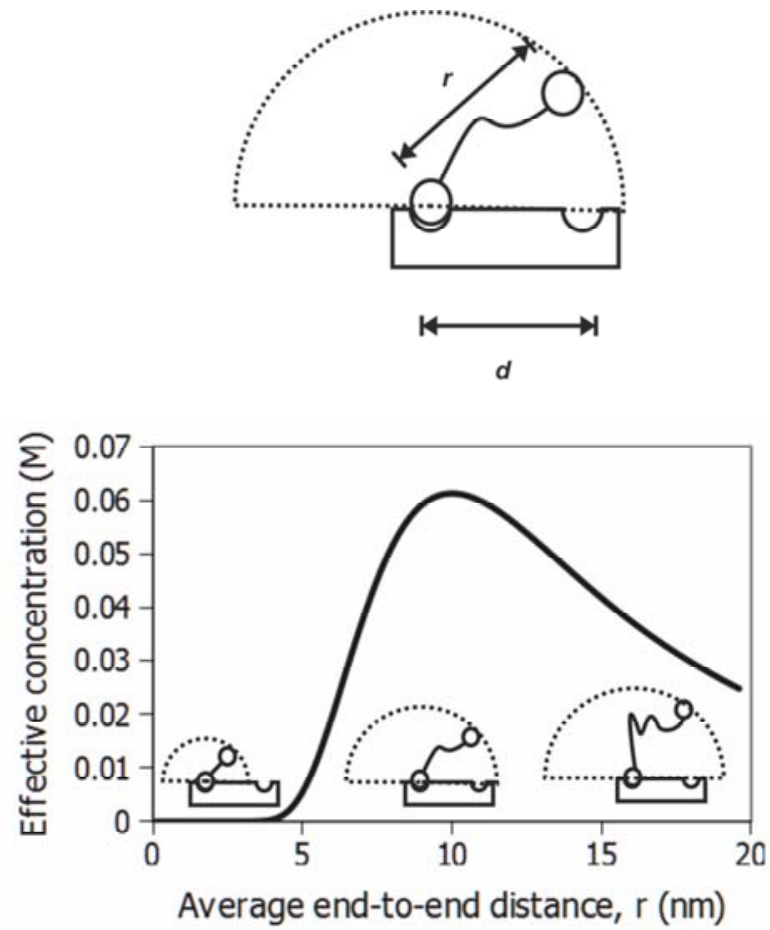

Figure 2.8 The effective concentration of host sites experienced by the unbound guest moiety depends on the properties and length, $r$, of the linker between the moieties, as well as the distance between binding sites $d$ (arbitrarily set to $10 \mathrm{~nm}$ ). In this example the host is supposed to restrict the probing volume to a half-sphere $(p=1 / 2)$.

\subsubsection{Interannular cooperativity}

Interannular cooperativity occurs when the first intramolecular binding has an effect in successive intramolecular events, as depicted in Figure 2.9. Thus, this type of cooperativity can only appear when two or more rings are formed as a consequence of the binding process. 


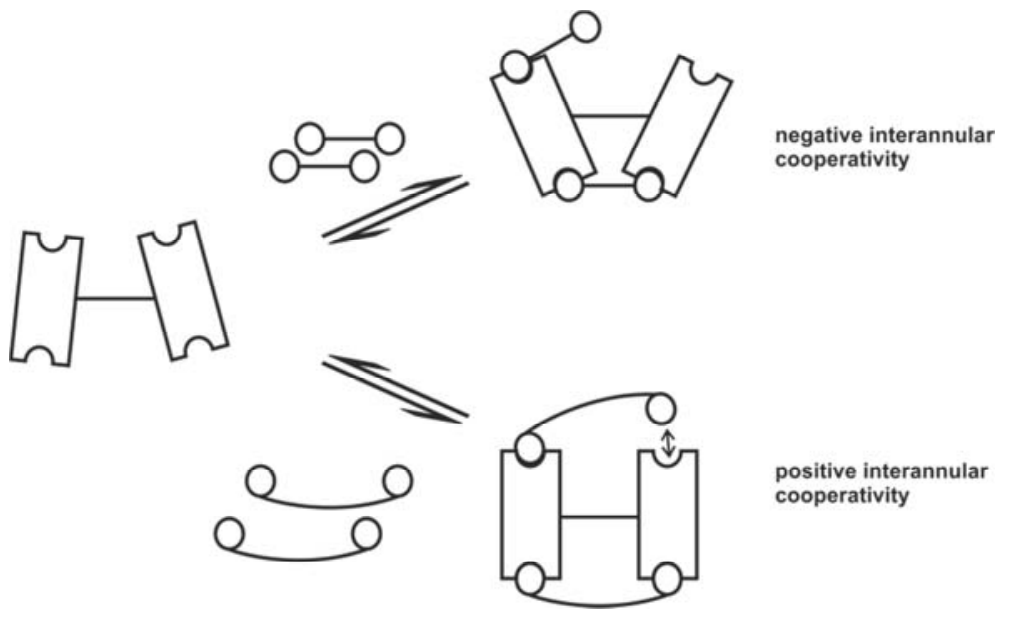

Figure 2.9 Interannular cooperativity.

In the case of the complex presented in Figure 2.10, the effect of this type of cooperativity can be assessed by determining the effective molarity for the different intramolecular events, $E M_{1}$ and $E M_{2}$, and comparing it to a reference effective molarity, $E M$. Thus, deviations from unity of the ratio $\gamma=$ $E M_{1} E M_{2} / E M$ indicate negative $(\gamma<1)$ or positive $(\gamma>1)$ interannular cooperativity.
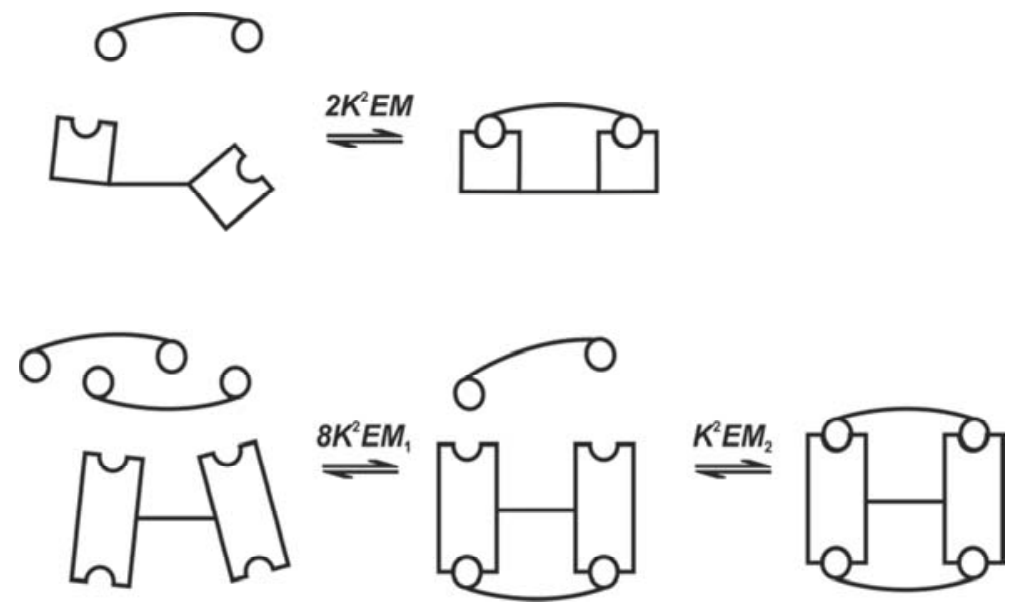

Figure 2.10 Determination of the effective molarities that characterize the presence of interannular cooperativity. 


\subsubsection{Other models for multivalent complexes}

The presented expression $K_{m}=\alpha \gamma K_{\sigma} K^{b} E M^{c}$ for the equilibrium constant generalizes many previously reported models of multivalency. ${ }^{2 a, 25}$ The product can be converted into an addition of contributions to the free energy of the complex:

$$
\begin{aligned}
& \Delta G_{m}^{o}=-R T \ln \left(K_{m}\right)=-R T(\underbrace{\ln (\alpha)}_{\text {allost }}+\underbrace{\ln (\gamma)}_{\text {iann }}+\underbrace{\ln \left(K_{\sigma}\right)}_{\text {deg }}+\underbrace{\ln (K)}_{\text {inter }}+ \\
& \underbrace{\ln \left(K^{b-1} E M^{c}\right)}_{\text {chel }}) \quad \text { (Eq. 2.3) }
\end{aligned}
$$

Then identical contributions in the expression can be identified with the ones reported in the work of Kitov and Bundle: ${ }^{25 e}$

$\Delta G_{b}^{o}=\underbrace{\Delta G_{\text {inter }}^{o}}_{\text {inter }}+\underbrace{(b-1) \Delta G_{\text {intra }}^{o}}_{\text {chel }}-\underbrace{R T \ln \left(\Omega_{b}\right)}_{\text {deg }}$

In this work independent binding sites were assumed, so the contributions from allosteric and interannular cooperativities were not considered. The identification of the intermolecular contribution is trivial. The complex was supposed to be bimolecular in this model ( $i=2$ in the generalized model), substituting the value of $c$ :

$(b-1) \ln (K E M)=(b-1) \Delta G_{\text {intra }}^{o}$

And the degeneracy term $\Omega_{b}$, which represents the multiplicity of possible microscopic arrangements leading to the same indistinguishable assembly, clearly plays the same role as $K_{\sigma}$ in the generalized model. In the paper of Kitov and Bundle an explicit expression for this degeneracy is only given for the most common binding geometries, a general method for calculating this number was reported later by Ercolani et al. ${ }^{25 d}$ This contribution is purely entropic and always enhances the binding affinity even in the case of only one possible bond. This effect has been also acknowledged in the work by Kiessling et al, ${ }^{12 a}$ where it is denominated as statistical rebinding, although the enhancement does not require fast rebinding kinetics as the word rebinding may suggest.

The thermodynamic model presented by Hamacek et al. ${ }^{25 b, c}$ can be compared in the same way. In this case two extra contributions to the free energy appear to describe the electrostatic interactions between adjacent ligands and/or 
metal ions in the complex. These contributions will modify the binding affinities of successive attachments and as such can be interpreted as an allosteric cooperativity phenomenon. The concept of interannular cooperativity is very recent and was not present in any other previous model, although enhanced binding due to this effect has been previously observed experimentally and however incorrectly interpreted as allosteric cooperativity. ${ }^{19,26}$

\subsubsection{Kinetics of multivalent complexes}

As it was discussed in the previous sections, multivalency can greatly enhance the thermodynamic stability of a complex. Moreover, a similar effect takes place regarding the kinetic stability. The association and dissociation rate constants of a multivalent complex can be estimated based on the kinetics of the monovalent interaction and the equilibrium constant of the multivalent assembly. ${ }^{2 b}$ For the case of a bimolecular complex it is considered to be bound when at least one of the guest moieties remains attached to a host site (see Figure 2.11). The equilibrium constant, $K^{\prime}$, of such subspecies (Figure $2.11 \mathrm{~B}$ ) can be easily determined using the previously presented model, since there is only one interaction no cooperativity effects are present and only the statistical factor must be taken into account, $K^{\prime}=K_{\sigma} K$. The off-rate of such complex will be identical to the off-rate of the intrinsic interaction, $k_{\text {off }}$, thus the enhanced stability can only be attributed to an increased on-rate, $k_{\text {on }}^{\prime}=K_{\sigma} k_{o n}$.

For the case of the multivalent complex the initial binding step (the intermolecular reaction) is identical to the previous case and the rate of association will be enhanced by the same factor. Since the stability of the multivalent complex can be known, an estimation for the observed off-rate can be obtained from these two quantities, $k_{o f f-m}=\frac{k_{o n}^{\prime}}{K_{m}}=k_{o n}\left(\alpha \gamma K^{b} E M^{c}\right)^{-1}$. 
A

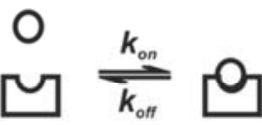

$K=k_{\text {on }} / k_{\text {ort }}$
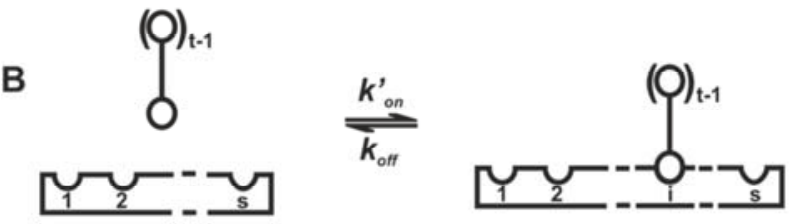

$K^{\prime}=s \cdot t \cdot K=k_{o n}^{\prime} / k_{o t \prime}$

C

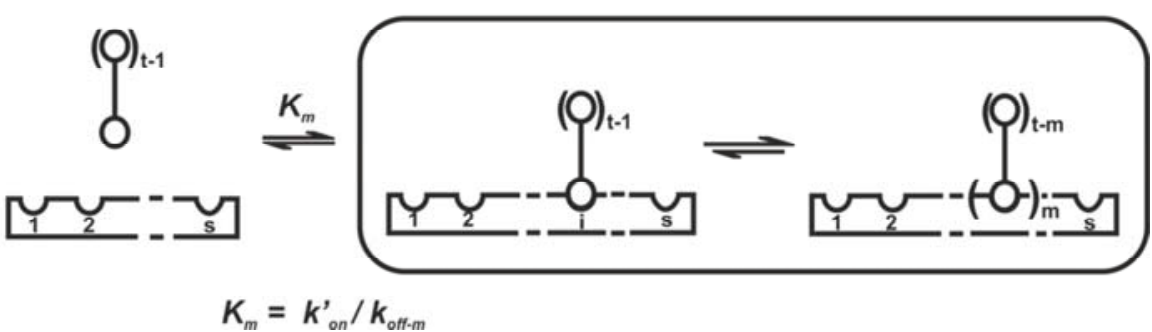

Figure 2.11 Estimating the kinetics of a multivalent complex. A) Intrinsic rates are determined from a monovalent model system. B) The equilibrium of a monovalently interacting multivalent pair allows the calculation of the on-rate. C) The off-rate for a multivalent complex is determined combining the two previous cases.

\subsubsection{Multivalency on surfaces}

The discussion so far has been focused on multivalent assemblies in solution, however, only few considerations are required in order to extend these models to the case where one of the species is attached to a surface (we will assume it is the host). The attachment can be accomplished by several strategies, from physisorption of unmodified host molecules to chemical linkage to a substrate or a monolayer, which often requires the introduction of reactive groups in the structure of the studied molecule. Two distinct situations can be described based on the surface density of the immobilized host compared to the size of a multivalent free guest. On the one hand, if the surface is sparsely covered by 
host molecules, the guest will only be able to complex to a single host, and the binding properties will resemble the binding of equivalent molecules both free in solution, which have been already discussed above. On the other hand, if the surface is densely covered by hosts, the guest molecule can complex binding sites from two or more different hosts. In this case the whole surface acts as a multivalent receptor, and the average distance between binding sites is more relevant to the binding properties than the structure of the host backbone. ${ }^{2 b, 27}$ Following this strategy, surfaces have been functionalized by densely packed monovalent host to construct multivalent receptors. ${ }^{28}$ The structure of the guest is usually the limiting factor for the maximum valency these surfaces can accommodate. $^{29}$

\subsection{Force spectroscopy}

The most studied aspect of multivalent systems has been so far their thermodynamic behavior. However, several biological processes such as endocytosis ${ }^{30}$ and the potential applications of multivalent building blocks rely on the kinetics of the interaction. ${ }^{15 b, 31}$ One of the biggest challenges to study the dynamics of multivalent assemblies is the fact that each additional bond can increase the lifetime of the complex by several orders of magnitude. This prevents the systematic application of usual techniques (NMR, FRET), since only one valency falls within the experimentally accessible range, lower order valencies being too fast and higher order valencies too slow to be measured. Additionally, not all the complexes are necessarily in the same binding state, that is, the signal obtained from ensemble measurements is likely a convolution of the signals corresponding to unbound, partially bound and totally bound complexes, weighted depending on their relative population in the sample. This latter problem is obviously not an issue when measuring at the single molecule level. This section will show how force spectroscopy (FS) is a technique able to measure properties of single bonds with stabilities spanning over several orders of magnitude. 


\subsubsection{Forced dissociation of supramolecular complexes}

The dissociation of a complex can be induced by applying a load to it, with its rate of dissociation (the inverse of the lifetime) increasing exponentially with the applied force, $f$, as predicted by Bell. ${ }^{32}$

$$
k_{o f f}(f)=k_{o f f}^{*} \exp \left[\frac{f \cdot x_{\beta}}{k_{B} T}\right]=k_{o f f}^{*} \exp \left[\frac{f}{f_{\beta}}\right]
$$

In this expression $k^{*}{ }_{\text {off }}$ is the intrinsic dissociation rate constant and $x_{6}$ the width of the energy barrier between bound and unbound state. Each interaction is usually characterized by a force $f_{b}$ and loading rate $\rho_{b}$ defined as:

$f_{\beta}=\frac{k_{B} T}{x_{\beta}}$

$\rho_{\beta}=f_{\beta} k_{o f f}^{*}$

This marked decrease of the lifetime with external applied force (see Figure 2.12) allows the study of several valences, since this effect can accelerate the unbinding of complexes that would take hours or months to spontaneously dissociate, making instead such events happen in fractions of a second. This inspired the development of force spectroscopy (FS), where mechanical stress is applied to a complex in a controlled manner in order to examine the properties of the bond. FS comprises several techniques, biomembrane force probe (BFP), magnetic (MT) or laser optical tweezers (LOT) and atomic force microscopy (AFM). All of them allow experimentalists to control and measure small forces in the range of $\mathrm{pN}$, as well as the loading rate, that is, how fast the forces are applied. Each technique offers different ranges of applicability, both in force (MT can measure forces as small as $\mathrm{FN}$, LOT and BFP can measure fractions of $\mathrm{pN}$, while $A F M$ is limited to forces over $10 \mathrm{pN}$ ) and loading rate (BFP $10^{1-4}$, LOT $10^{2-3}$ and $\left.A F M \sim 10^{2-8} \mathrm{pN} / \mathrm{s}\right){ }^{33}$ The relationship of the rupture forces with the loading rate was first studied by Evans and Ritchie, ${ }^{34}$ and provides information such as the kinetic off-rate of the complex and the width of the potential. The most probable rupture force, $f^{*}$, scales logarithmically with the loading rate, $\rho$.

$f^{*}(\rho)=\frac{k_{B} T}{x_{\beta}} \ln \left(\frac{\rho x_{\beta}}{k_{B} T k_{o f f}^{*}}\right)=f_{\beta} \ln \left(\frac{\rho}{\rho_{\beta}}\right)$ 
The rest of this discussion will be focused on AFM-based $\mathrm{FS}^{35}$ but all considerations are general and valid for the other mentioned FS techniques as well.

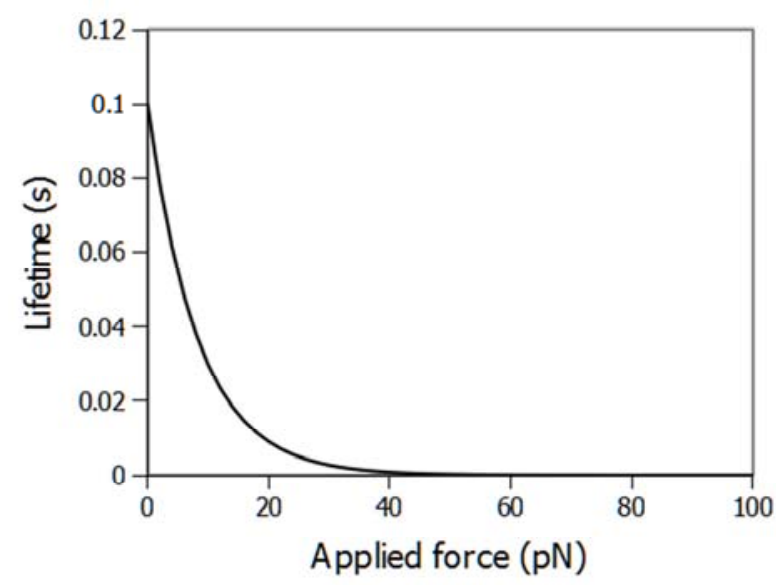

Figure 2.12 Lifetime of a complex versus applied force. The parameters describing the complex of the plot are $k^{*}{ }_{\text {off }}=10 \mathrm{~s}^{-1}$ and $x_{\beta}=0.5 \mathrm{~nm}$. The same values will be used in the following figures unless stated otherwise.

\subsubsection{AFM dynamic force spectroscopy}

To study a host-guest complex using FS both the host and the guest moieties have to be secured each to a different surface, either to a supporting flat substrate or to the surface of the tip of an AFM cantilever. The distance between these two functionalized surfaces is controlled by means of a piezoelectric crystal. A laser beam is used to detect the deflection of the cantilever with $\mathrm{nm}$ precision. Usually the measurements are performed in a liquid medium to avoid capillary forces between the tip and the substrate, in many cases aqueous solutions but also organic solvents such as ethanol, ${ }^{36}$ or $\mathrm{DMF}^{37}$ may be used. The host- and guest-functionalized surfaces are brought into contact and then the separation distance between them is gradually increased at a determined speed $v$. The interaction between the two surfaces is detected as a negative cantilever deflection until the energy accumulated in the cantilever is enough to break the attractive interaction. This rupture is indicated by a sudden change in the deflection signal. Since the spring constant of the 
cantilever $(K c)$ can be calibrated using diverse methods, ${ }^{38}$ the deflection values can be translated into force values. The data obtained from this procedure is usually plotted as a force-distance curve (see Figure 2.13).

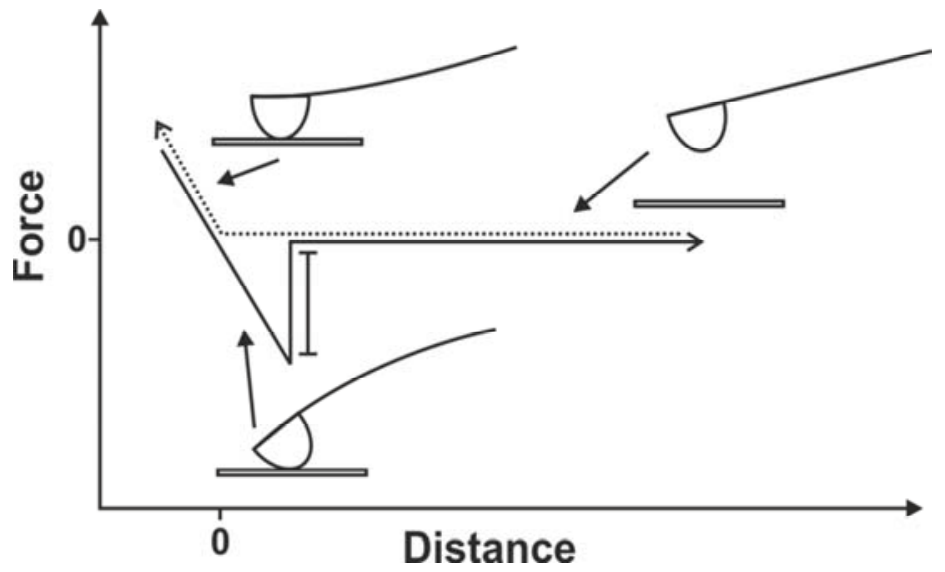

Figure 2.13 Ideal force-distance curve, showing the approach and retract cycle.

However, if the host and guest moieties are directly linked to the surfaces several problems can arise. Firstly, the number of host-guest pairs in the contact area is not determined and depending on the radius of the tip and the density of functionalization can be up to several tens of complexes, which makes the study of the interaction at the single molecule level difficult if not impossible. The usage of mixed monolayers, ${ }^{39}$ careful control of functionalization times or thoroughly testing the dependence of the functionalization density on the employed chemical functionalization route ${ }^{40}$ enables to dilute the presence of the studied molecules in the contact patch. Secondly, the measured rupture force is likely to be affected by non-specific factors such as Van der Waals and hydration forces between the tip and the substrate when they are in close proximity. Finally, some host-guest species, particularly biomolecules, require a certain degree of freedom to be able to form a complex, which may not be provided by a direct attachment to the surface. These problems have motivated the introduction of long polymeric tethers between the studied moieties and the tip surface and/or the substrate. ${ }^{41}$ Such linkers provide enough freedom of rotation so complexes will be formed and effectively resolve non-specific ruptures from specific host-guest interactions, which occur at distances from the surface comparable to the 
length of the employed polymer chain (see Figure 2.14). Moreover, the characteristic non-linear elasticity of a polymer chain enables the discrimination of single and multiple simultaneous ruptures by fitting the non-linear region of the force-distance curve to an appropriate model (worm-like chain, freelyjointed chain) describing the chosen polymer. ${ }^{42}$ However, this non-linear behavior influences the effective loading rate that the bond feels, so using the externally imposed loading rate $\left(\rho=K_{c} v\right)$ will yield erroneous results. For this reason the slope of the force-distance curve just before the rupture is measured and used to calculate the instantaneous loading rate $\left(\rho_{\text {inst }}\right)$ that was exerted on the bond at that moment.

Since thermal energy is typically comparable to the binding energy of supramolecular complexes, hundredths or thousands of these force-distance curves must be measured in order to determine a reliable value for the rupture force. The curves are usually selected to discard unbinding events originating from non-specific interactions or from several multiple interactions. Different attempts have been made to automate this task by using computer algorithms. ${ }^{43}$ However, the uniqueness of each studied system and often the differences in data formats between commercial AFM setups have prevented the acceptance of a universal solution to this aspect. ${ }^{44}$ Once a significant amount of adequate curves has been selected, a statistical analysis of the rupture force values (and in the case of polymeric linkers, instantaneous loading rate values) is performed, typically by constructing a histogram and fitting a Gaussian distribution to its shape. Alternative treatments, such as the use of kernel density estimations (KDE), have been proposed and offer improved results for low number of measurements. ${ }^{45}$ In any case, these procedures enable the determination of the most-probable rupture force $f$ and loading rate $\rho$. 


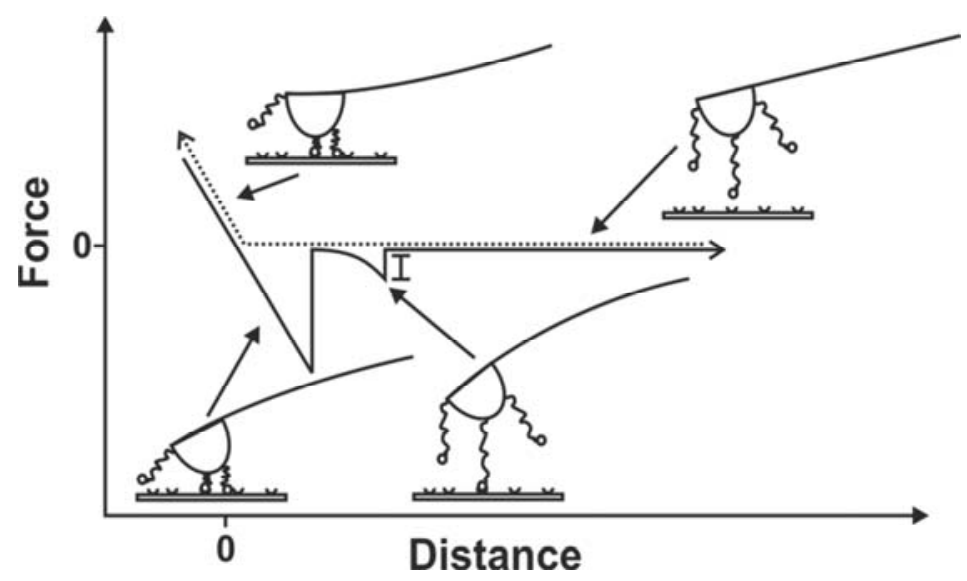

Figure 2.14 Ideal force-distance curve using a long linker to anchor the guest moiety. A secondary rupture is observed at a distance from the surface similar to the length of the employed linker.

\subsubsection{Beyond conventional force spectroscopy}

In the past years several theoretical developments and experiments that extend the potential applications of force spectroscopy techniques have been reported. As was discussed above, the usual information obtained from a DFS experiment is the dissociation rate constant. However, the standard Evans model does not provide other interesting quantities such as the free energy of activation $\left(\Delta G^{\ddagger}\right)$. Revisions to the theory of forced dissociation that offer an estimate for this energy have been proposed. ${ }^{46}$. The use of Jarzynski's equality, ${ }^{47}$ which allows to extract thermodynamic parameters from nonequilibrium measurements, has also been explored in connection with $\mathrm{FS}^{48}$ Furthermore, the association rate constant can be estimated from FS experiments, ${ }^{49}$ as well as the refolding pathways of proteins. ${ }^{50}$ Optimizing the conditions for successful binding ${ }^{51}$ enables the construction of forcerecognition maps, which can be combined with traditional AFM topography to locate the position of receptors on cell membranes. ${ }^{52}$ The combination of FS with other techniques, such as fluorescence, offers the possibility not only of overlay recognition maps with fluorescence images, ${ }^{53}$ but also of investigating the effects of mechanical stress in fluorescence intensity. ${ }^{54}$ Moreover, the application of force has been found to activate chemical reactions. ${ }^{55}$ 


\subsection{Rupture of multiple bonds}

Up to this point the presented discussion was aimed at the study of monovalent binding. Several approaches have been made to extend the EvansRitchie model to the rupture of multiple bonds and will be briefly discussed here. In all cases the monovalent system will be the same, characterized by the constants $\mathrm{k}_{\text {off }}^{*}=10 \mathrm{~s}^{-1}$ and $\mathrm{x}_{\beta}=0.5 \mathrm{~nm}$.

\subsubsection{Poisson model}

The simplest model, Poisson analysis, ${ }^{56}$ assumes a linear scaling of the unbinding force (see Figure 2.15).

$f^{*}(N)=N f_{\text {mono }}^{*}$

\section{Poisson scaling}

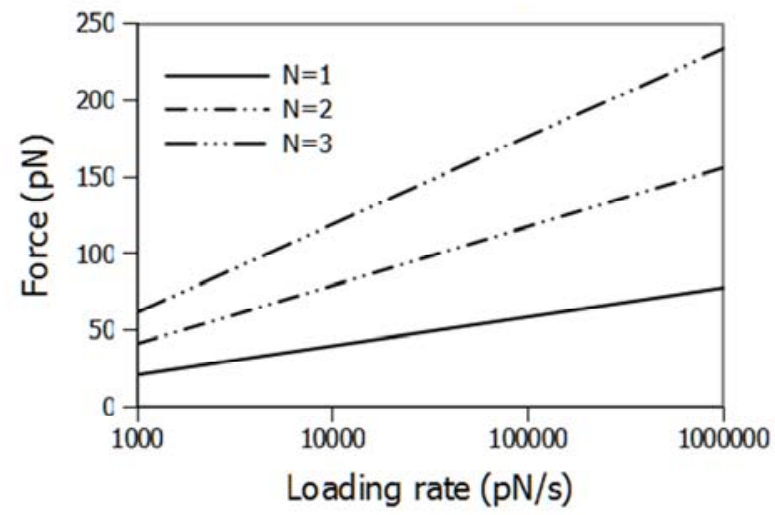

Figure 2.15 Scaling of rupture forces according to Poisson analysis for $\mathrm{N}=1,2,3$ parallel bonds.

However, this model implies that all bonds are in the same energy state and subject to the same pulling force and loading rate, hence they break at the exact same instant. ${ }^{57}$ This is the typical situation for macroscopic ruptures where the width of the rupture force distribution is small compared with the rupture force of the complexes. However, that is certainly not the case for most supramolecular complexes, and even the previously mentioned results showing linear scaling have been more recently reinterpreted as originating from differences in instantaneous loading rate. ${ }^{58}$ 


\subsubsection{Seifert model}

A more detailed paper by Seifert ${ }^{57 a}$ studies several possible experimental situations considering the combined stiffness of the cantilever and the linker molecules $\left(K_{t}\right)$, as well as the possibility of reforming broken bonds (reversibility). Different regimes are identified by comparison with the quantity $\mu$, defined as:

$\mu=\frac{K_{t} v x_{\beta}}{k_{B} T k_{o f f}^{*}}=\frac{\rho}{\rho_{\beta}}$

The rupture force for $\mathrm{N}$ irreversible bonds would be:

$f_{\text {soft-irrev }}^{*}(N) \sim\left\{\begin{array}{cc}f_{\beta} \mu \ln (N) & \text { for } \mu<1 \\ f_{\beta} \mu \ln \left(\frac{N}{\mu}\right) & \text { for } 1 \leq \mu \leq N \\ f_{\beta} N \ln \left(\frac{\mu}{N}\right) & \text { for } N<\mu\end{array}\right.$

$f_{\text {stiff-irrev }}^{*}(N) \sim \begin{cases}f_{\beta} \mu N & \text { for } \mu \leq 1 \\ f_{\beta} N \ln (\mu) & \text { for } \mu>1\end{cases}$

And in the case of reversible bonds (where $\mathrm{N}$ is the number of bonds in equilibrium without loading):

$f_{\text {soft-rev }}^{*}(N) \sim \begin{cases}f_{\beta}(\mu N)^{\frac{1}{2}} & \text { for } \mu \leq N \\ f_{\beta} N \ln \left(\frac{\mu}{N}\right) & \text { for } N<\mu\end{cases}$

$f_{\text {stiff-rev }}^{*}(N) \sim \begin{cases}f_{\beta} \mu^{\frac{1}{2}} N & \text { for } \mu \leq 1 \\ f_{\beta} N \ln (\mu) & \text { for } \mu>1\end{cases}$

Notice that reversibility only plays a role when a bond is loaded at rates lower than the characteristic loading rate $\left(\rho / N \leq \rho_{\beta}\right)$. If this condition does not hold, and for the case of a stiff cantilever, the results of Poisson analysis are recovered. Thus, only the case of an irreversible bond connected to a soft cantilever is shown in Figure 2.16. 


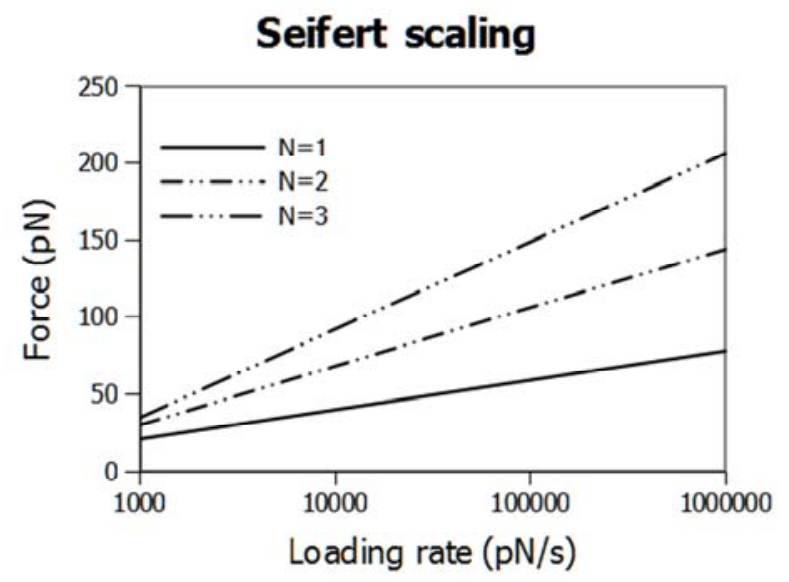

Figure 2.16 Scaling of rupture force according to Seifert model for an irreversible bond connected to a soft cantilever for $\mathrm{N}=1,2,3$ parallel bonds.

\subsubsection{Reliability model}

Alternatively, Tees' model ${ }^{59}$ considers all the bonds to be loaded with the same force, but each one breaks following Bell's model. The model is based in reliability theory, ${ }^{60}$ a method usually applied to calculate failure probabilities of electronic components and networks. The derived expression predicts that the rupture force of a multivalent complex should scale harmonically with the number of bonds.

$f^{*}(N)=f_{\text {mono }}^{*}\left(1+\frac{1}{2}+\cdots+\frac{1}{N}\right)$ 


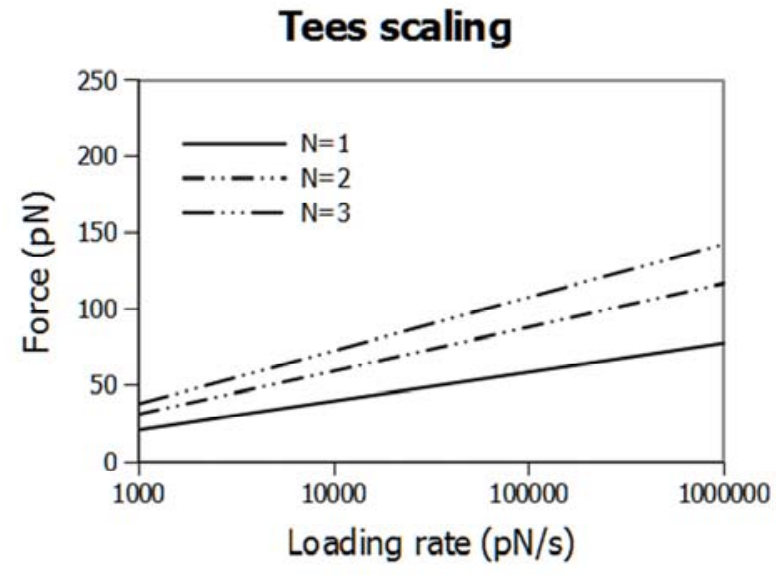

Figure 2.17 Scaling of rupture force according to Tees model for $\mathrm{N}=1,2,3$ parallel bonds.

\subsubsection{Williams parallel and zipper models}

Finally, Williams has proposed two models to describe two different geometries and rupture mechanisms of the complex. ${ }^{57 b}$ The zipper model considers the case where the force is mainly exerted over one of the bonds until it breaks and then the next bond receives the load. For this type of binding the force required to break the compound bond is only slightly higher than the one required to break a single bond.

$f_{\text {zipper }}^{*}(N)=f_{\text {mono }}^{*}+f_{\beta} \ln (N)$

On the other hand, the parallel model, presented in the same article, assumes the load is distributed equally among all bonds. In this case the expression for the rupture force needs to be solved numerically.

$\frac{\rho_{\beta}}{\rho}=\sum_{i=1}^{N} \frac{1}{i^{2}} \exp \left(-\frac{f_{\text {parallel }}^{*}}{i f_{\beta}}\right)$ 


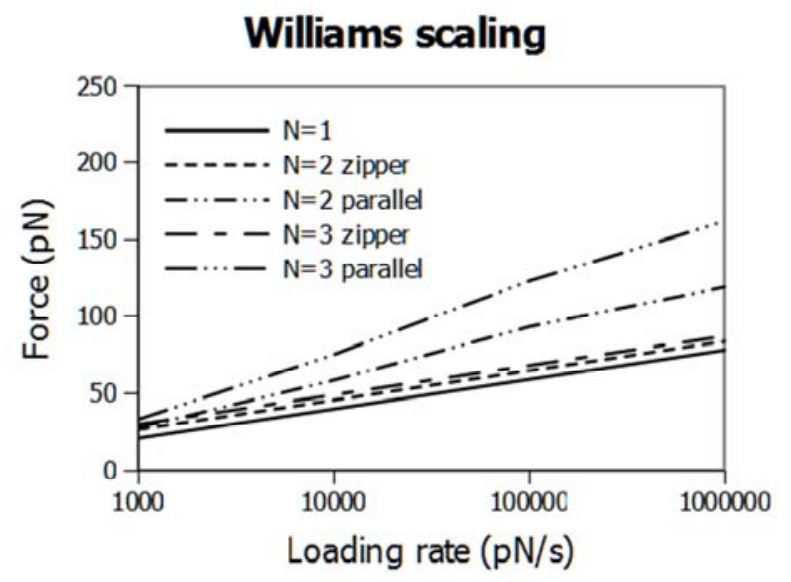

Figure 2.18 Scaling of rupture forces according to Williams zipper (loading in series) and parallel models for $\mathrm{N}=1,2,3$ parallel bonds.

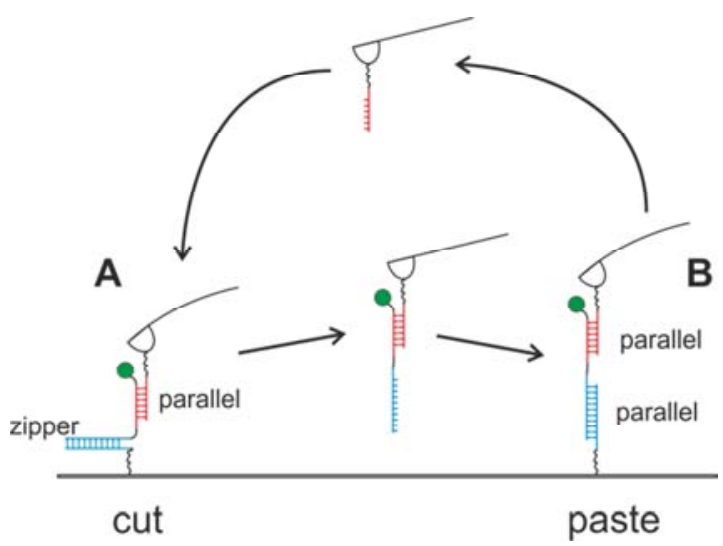

Figure 2.19 Cut\&paste of DNA decorated particles. A) "Cut": although the blue DNA double strand has more base pairs (bigger $N$ ) than the red strand, they are loaded in different configuration, zipper and parallel, respectively. Thus the blue connection ruptures at lower force than the red one. B) "Paste": In this case both double strands are loaded in the parallel configuration, so the shorter one (red) is ruptured first. 
The contrast between the rupture force of these two different geometries is clear (see Figure 2.18) and can be useful for experimental applications. The group of Gaub has been able for example to "cut" nanoparticles from an area of a substrate and then "paste" them into another area by using DNA handles that were loaded in zipper and parallel configurations (see Figure 2.19). ${ }^{61}$

\subsubsection{Comparing theoretical models of multiple ruptures}

From the different expressions presented above it seems clear that the predicted value for the rupture force of a multivalent bond will not be the same depending on the chosen model. A comparison between the predicted rupture forces is presented in Figure 2.20.

The validity of some of these models is seemingly ambiguous when fitting or modeling the experimentally measured values, also because the intrinsic uncertainty of the most probable rupture force that is characteristic of performing these experiments. In the case of $\mathrm{N}=2$ Seifert, Tees and Williams parallel models predicted rupture forces would differ by ca. $10 \mathrm{pN}$, which is often less than the standard deviation of the measured most probable rupture force using AFM. However, Poisson and Williams zipper predictions differ enough to be measurable. Moreover, there are additional differences that can be assessed from the trend of rupture forces versus loading rate and that are not apparent from this figure. If parallel ruptures were to be measured experimentally and analyzed using Evans model, different values of the width of the potential $x_{B-N}$ and the dissociation constant $k_{\text {off- } N}^{*}$ would be obtained from fits to the data. The expected values for these quantities assuming each model is correct are presented in Figure 2.21 and Figure 2.22. 


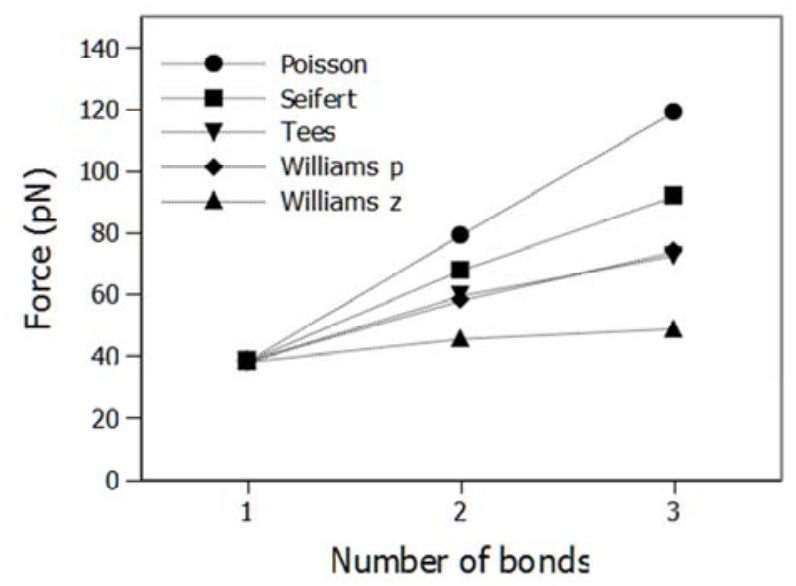

Figure 2.20 Comparison of the predicted rupture force for $\mathrm{N}=\mathbf{2}$ and $\mathrm{N}=\mathbf{3}$ according to the different models. All the values are for a loading rate of $10000 \mathrm{pN} / \mathrm{s}$. Tees and Williams parallel symbols are overlapping.

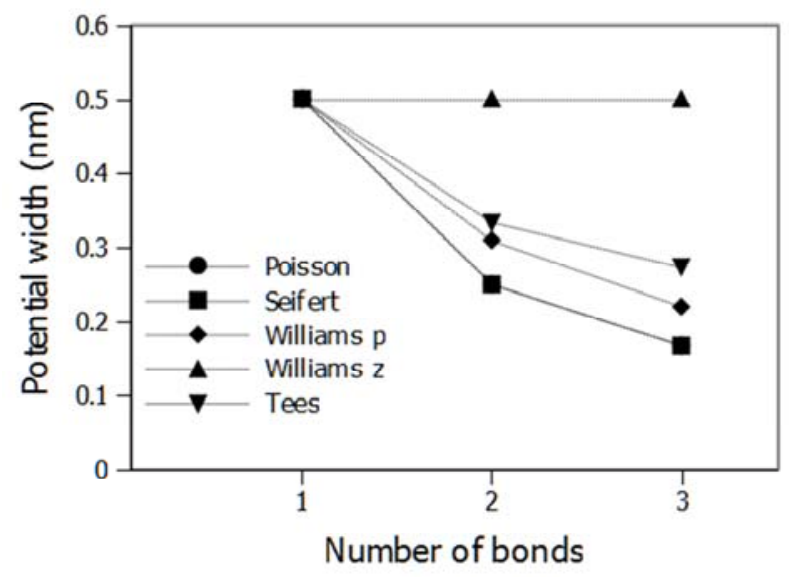

Figure 2.21 Width of the potential $\left(x_{6-N}\right)$ obtained from fits of Evans model to the predicted rupture force - loading rate trend of each model. Poisson and Seifert symbols are overlapping.

It is found that Williams zipper model predicts a potential width independent of the number of bonds, which is reasonable since the main assumption of the model was that each bond is broken successively. On the other hand, the rest 
of the models predict a decrease of the fitted $\mathrm{x}_{\beta}$, scaling as $1 / \mathrm{N}$ for Poisson and Seifert models, and more weakly in the case of Tees and Williams parallel models. Considering the unforced dissociation rates more differences can be found. The Seifert model shows an independent dissociation rate with the number of bonds. Williams zipper and parallel, Tees and Poisson models predict an increased stability with increasing number of bonds, which, at least qualitatively, is the expected behavior.

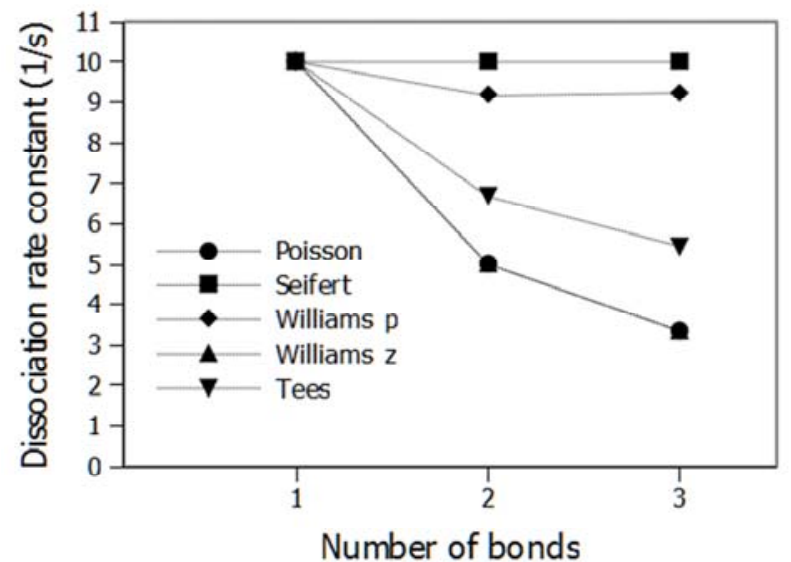

Figure 2.22 Dissociation rate at zero force $\left(k^{*}{ }_{\text {off-N }}\right)$ obtained from fits of Evans model to the predicted rupture force - loading rate trend of each model. Poisson and Williams zipper symbols are overlapping.

Unfortunately, experimental studies of multiple ruptures are scarce. An extensive search of literature was performed to find examples of multiple ruptures studied by force spectroscopy in order to compare them to the presented theories. In many cases multiple peaks were interpreted as multiple unbinding, but no further analysis was performed on them. ${ }^{62}$ Several papers report a linear scaling of the rupture force with the number of bonds, ${ }^{39 a, 63}$ when not directly assuming that a single force peak originates from multiple bonds that scale linearly. ${ }^{64}$

On the other hand, sublinear scaling of the rupture force with the number of bonds has been reported too. In the case of Ru coordination complexes force peaks were found at 99, 165 and $228 \mathrm{pN} .{ }^{65}$ The authors found these values to compare well to Monte Carlo simulations of the rupture, although none of the models discussed here describe properly this force sequence. Another example 
is the study of concanavalin A - mannose, where ruptures of 46, 68, and $85 \mathrm{pN}$ were detected. ${ }^{66}$ A satisfactory comparison with Williams zipper and Tees models is reported in the same paper. However, measurements of MUC1 antigen-antibody complexes in parallel appear to scale according to Williams parallel model. ${ }^{58,67}$ Finally, the interaction between $\mathrm{C}_{60}$ and porphyrin tweezers has been reported to be over two times stronger than the interaction between $\mathrm{C}_{60}$ and porphyrin, ${ }^{68}$ which is to our knowledge the only existing report of overlinear scaling of forces, and is clearly not incorporated by any of the proposed models.

As it was discussed above, examining the trends of the rupture forces versus loading rates could help to decide about the applicability of each model, by comparing the fitted dissociation rate constant and potential width. Unfortunately, only two experimental studies reporting the force rupture values at more than one loading rate were found. ${ }^{67-68}$ Considering the completely different behavior of the double bond force reported therein (sublinear and over-linear, respectively) the need of more studies addressing this question is evident.

\subsubsection{Additional effects on multiple ruptures, linkers and fast rebinding}

Some authors have pointed out that the models described above assume equal loading for all the bonds, which in turn means all the connecting linkers are of the same length. ${ }^{69}$ A rupture force for a double bond of less than two times the rupture force of a single interaction is predicted by a model taking in account the polydispersity of the linkers. It is important to remark, though, that the precise force value of this double bond rupture will shift towards lower forces with increasing differences of length between the two loaded linkers. An equivalent effect can be also introduced by microscopic irregularities on the tip and/or substrate surfaces, which can cause differences in the load even in the case of pulling from linkers of identical length. Experiments where this model is successfully applied have been recently reported. ${ }^{70}$

Moreover, in all cases these models assume that the monovalent interaction is being loaded out of equilibrium, that is, Evans model is applicable in the studied range of loading rate. While this is the case for most part of the biological complexes studied in the past, some weak supramolecular interactions exhibit very fast kinetics which cannot be described in this framework. ${ }^{39 a}{ }^{71}$ In such 
situations the complex is loaded in a quasi-equilibrium state, which will be affected by the linkage of the moieties, since it determines the effective concentration of binding sites that the linked moieties experience (see Figure 2.23). Thus, in experiments where fast rebinding takes place the choice of an attachment strategy can dramatically change the results of FS studies.
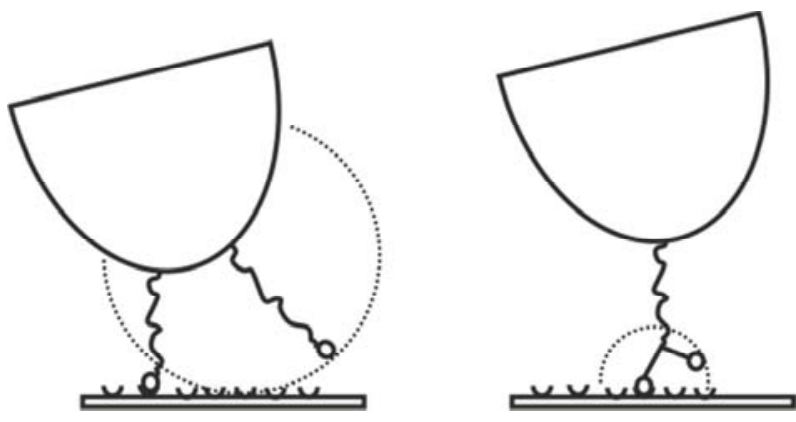

Figure 2.23 In a divalent complex, the effective concentration experienced by the second guest moiety while the first remains attached is determined by the type, length and geometry of the linkers employed to connect the moieties to the tip.

\subsection{Concluding remarks}

The thermodynamic behavior of multivalent assemblies in solution and surfaces seems a well-established matter, apart from minor discrepancies mostly motivated by the precise definition of the concept of cooperativity. On the other hand, the kinetics of such assemblies have not received much attention. It has been shown how force spectroscopy is a potentially useful tool to investigate this aspect. However, few studies addressing this phenomenon have been reported, and many lack enough data to validate or discriminate between the several existing theoretical models. 


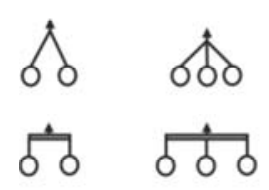

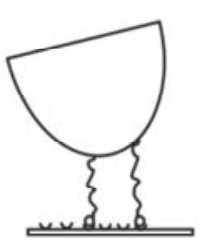

A

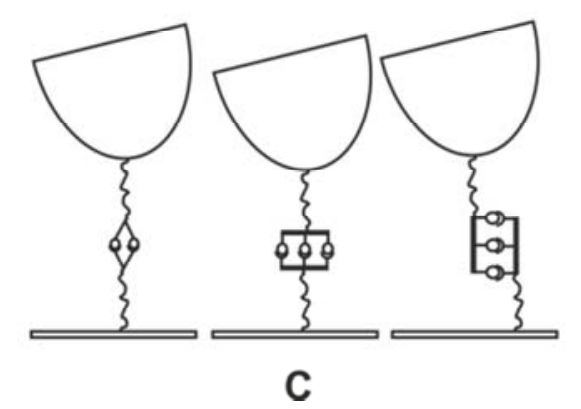

Figure 2.24 Possible attachment strategies for the study of multivalent complexes. A) parallel pulling may introduce undesired effects due to differences in linker length or anchoring point, as well as effective concentration for interactions where rebinding occurs B) loading the complex using a single linker eliminates or minimizes these effects C) double tether approach can be used, providing more solution-like conditions and an effectively longer linker.

We propose some guidelines that should provide optimal conditions for this kind of experiments (see Figure 2.24). (i) Long polymeric linkers should be used, as the relatively short linkers (or even direct attachment) employed in the past made the quantification of the number of bonds difficult. Additionally, they do not allow an estimation of the instantaneous loading rate that is applied to the bond, which may be affected even by the elasticity of the investigated protein in the case of biological samples. (ii) Experiments where the complex is ruptured while pulling from a single linker should be pursued, as the differences in linker length due to the usage of polydisperse linkers can affect the rupture force. Very low polydispersity index linkers are difficult and expensive to obtain, especially if they present reactive end-groups to connect them to the substrates and studied molecules. Even if such linkers were available, the attachment point in the tip and the differences in elongation caused by irregularities of the tip and the supporting substrate are unavoidable. The construction of a molecular scaffold which presents the desired number of moieties and one anchoring point to the linker can prevent such problems. (iii) 
Finally, in the case of fast rebinding complexes the dissociation rate is expected to depend on the equilibrium constant of the whole complex, which in turn depends on the chelate and interannular cooperativity effects. The use of similar molecular scaffolds when comparing multivalent complexes in solution and in a FS experiment is in this case is the only way to achieve a situation where the cooperativity of the complex is properly defined.

\subsection{References and notes}

1. (a) Kiessling, L. L.; Lamanna, A. C., Multivalency in Biological Systems. In Chemical Probes in Biology, Schneider, M. P., Ed. Kluwer Academic Publishers: Dordrecht, 2003; (b) Mulder, A.; Huskens, J.; Reinhoudt, D. N., Org. Biomol. Chem. 2004, 2, 3409-24; (c) Badjic, J. D.; Nelson, A.; Cantrill, S. J.; Turnbull, W. B.; Stoddart, J. F., Acc. Chem. Res. 2005, 38, 723-32.

2. (a) Ercolani, G., J. Am. Chem. Soc. 2003, 125, 16097-103; (b) Huskens, J.; Mulder, A.; Auletta, T.; Nijhuis, C. A.; Ludden, M. J.; Reinhoudt, D. N., J. Am. Chem. Soc. 2004, 126, 6784-97; (c) Kane, R. S., Langmuir 2010, 26, 8636-40.

3. Mammen, M.; Choi, S.-K.; Whitesides, G. M., Angew. Chem. Int. Ed. 1998, 37, 2754-94.

4. Rowan, S. J.; Cantrill, S. J.; Cousins, G. R. L.; Sanders, J. K. M.; Stoddart, J. F., Angew. Chem. Int. Ed. 2002, 41, 898-952.

5. Yamada, S.; Pokutta, S.; Drees, F.; Weis, W. I.; Nelson, W. J., Cell 2005, 123, 889-901.

6. Gumbiner, B. M., Cell 1996, 84, 345-57.

7. Lundquist, J. J.; Debenham, S. D.; Toone, E. J., J. Org. Chem. 2000, 65, 8245-50.

8. Pincet, F.; Le Bouar, T.; Zhang, Y.; Esnault, J.; Mallet, J. M.; Perez, E.; Sinay, P., Biophys. J. 2001, 80, 1354-8.

9. Liu, Q. P.; Sulzenbacher, G.; Yuan, H.; Bennett, E. P.; Pietz, G.; Saunders, K.; Spence, J.; Nudelman, E.; Levery, S. B.; White, T.; Neveu, J. M.; Lane, W. S.; Bourne, Y.; Olsson, M. L.; Henrissat, B.; Clausen, H., Nat. Biotechnol. 2007, 25, 454-64.

10. Heldin, C.-H., Cell 1995, 80, 213-23.

11. (a) Cuesta, Á. M.; Sainz-Pastor, N.; Bonet, J.; Oliva, B.; Álvarez-Vallina, L., Trends Biotechnol. 2010, 28, 355-62; (b) Thakur, A.; Lum, L., Curr. Opin. Mol. Ther. 2010, 12, 340 - 9. 
12. (a) Kiessling, L. L.; Gestwicki, J. E.; Strong, L. E., Angew. Chem. Int. Ed. 2006, 45, 2348-68; (b) Gestwicki, J. E.; Strong, L. E.; Kiessling, L. L., Chem. Biol. 2000, 7, 583-91.

13. Banerjee, I. A.; Yu, L.; Matsui, H., J. Am. Chem. Soc. 2003, 125, 9542-3.

14. Bhagawati, M.; Lata, S.; Tampé, R.; Piehler, J., J. Am. Chem. Soc. 2010, 132, 5932-3.

15. (a) Wang, X.; Mart, R. J.; Webb, S. J., Org. Biomol. Chem. 2007, 5, 2498505; (b) Vico, R. V.; Voskuhl, J.; Ravoo, B. J., Langmuir 2010, 27, 1391-7.

16. Kostiainen, M. A.; Kasyutich, O.; Cornelissen, J. J. L. M.; Nolte, R. J. M., Nat. Chem. 2010, 2, 394-9.

17. Ling, X. Y.; Phang, I. Y.; Schönherr, H.; Reinhoudt, D. N.; Vancso, G. J.; Huskens, J., Small 2009, 5, 1428-35.

18. Yonetani, T.; Laberge, M., Biochim. Biophys. Acta 2008, 1784, 1146-58.

19. Shinkai, S.; Ikeda, M.; Sugasaki, A.; Takeuchi, M., Acc. Chem. Res. 2001, 34, 494-503.

20. Ercolani, G.; Schiaffino, L., Angew. Chem. Int. Ed. 2011, 50, 1762-8.

21. Jacobson, H.; Beckmann, C. O.; Stockmayer, W. H., J. Chem. Phys. 1950, $18,1607-12$.

22. Winnik, M. A., Chem. Rev. 1981, 81, 491-524.

23. Gargano, J. M.; Ngo, T.; Kim, J. Y.; Acheson, D. W. K.; Lees, W. J., J. Am. Chem. Soc. 2001, 123, 12909-10.

24. Krishnamurthy, V. M.; Semetey, V.; Bracher, P. J.; Shen, N.; Whitesides, G. M., J. Am. Chem. Soc. 2007, 129, 1312-20.

25. (a) Zeckert, K.; Hamacek, J.; Rivera, J. P.; Floquet, S.; Pinto, A.; Borkovec, M.; Piguet, C., J. Am. Chem. Soc. 2004, 126, 11589-601; (b) Hamacek, J.; Borkovec, M.; Piguet, C., Chemistry 2005, 11, 5217-26; (c) Hamacek, J.; Borkovec, M.; Piguet, C., Chemistry 2005, 11, 5227-37; (d) Ercolani, G.; Piguet, C.; Borkovec, M.; Hamacek, J., J. Phys. Chem. B 2007, 111, 12195-203; (e) Kitov, P. I.; Bundle, D. R., J. Am. Chem. Soc. 2003, 125, 16271-84.

26. Ayabe, M.; Ikeda, A.; Kubo, Y.; Takeuchi, M.; Shinkai, S., Angew. Chem. Int. Ed. 2002, 41, 2790-2.

27. Voskuhl, J.; Stuart, M. C. A.; Ravoo, B. J., Chem-Eur. J. 2010, 16, 2790-6.

28. Ludden, M. J. W.; Reinhoudt, D. N.; Huskens, J., Chem. Soc. Rev. 2006, 35, $1122-34$.

29. (a) Thompson, D., Langmuir 2007, 23, 8441-51; (b) Nijhuis, C. A.; Yu, F.; Knoll, W.; Huskens, J.; Reinhoudt, D. N., Langmuir 2005, 21, 7866-76; (c) Nijhuis, C. A.; Huskens, J.; Reinhoudt, D. N., J. Am. Chem. Soc. 2004, 126, 12266-7.

30. (a) Miliaras, N.; Wendland, B., Cell Biochem. Biophys. 2004, 41, 295318; (b) Bache, K. G.; Raiborg, C.; Mehlum, A.; Stenmark, H., J. Biol. Chem. 2003, 278, 12513-21. 
31. Banerjee, D.; Liu, A. P.; Voss, N. R.; Schmid, S. L.; Finn, M. G., ChemBioChem 2010, 11, 1273-9.

32. Bell, G. I., Science 1978, 200, 618-27.

33. In the case of AFM, the accesible loading rates depend on the stiffness of the cantilever and the linker. For a given cantilever the loading rate typically spans 3 orders of magnitude, limited by viscous drag of the medium. The usage of soft polymeric linkers limits the loading rate to values under $1000 \mathrm{nN} / \mathrm{s}$ independently of the stiffness of the cantilever.

34. Evans, E.; Ritchie, K., Biophys. J. 1997, 72, 1541-55.

35. Hugel, T.; Seitz, M., Macromol. Rapid Commun. 2001, 22, 989-1016.

36. (a) Eckel, R.; Ros, R.; Decker, B.; Mattay, J.; Anselmetti, D., Angew. Chem. Int. Ed. 2005, 44, 484-8; (b) Walther, K. A.; Gräter, F.; Dougan, L.; Badilla, C. L.; Berne, B. J.; Fernandez, J. M., Proc. Natl. Acad. Sci. U.S.A. 2007, 104, 7916-21.

37. Cuenot, S.; Gabriel, S.; Jérôme, R.; Jérôme, C.; Fustin, C.-A.; Jonas, A. M.; Duwez, A.-S., Macromolecules 2006, 39, 8428-33.

38. (a) Hutter, J. L.; Bechhoefer, J., Rev. Sci. Instrum. 1993, 64, 1868-73; (b) Sader, J. E.; Chon, J. W. M.; Mulvaney, P., Rev. Sci. Instrum. 1999, 70, 3967-9; (c) Gibson, C.; Watson, G.; Myhra, S., Nanotechnology 1996, 7, 259-62.

39. (a) Auletta, T.; de Jong, M. R.; Mulder, A.; van Veggel, F. C.; Huskens, J.; Reinhoudt, D. N.; Zou, S.; Zapotoczny, S.; Schönherr, H.; Vancso, G. J.; Kuipers, L., J. Am. Chem. Soc. 2004, 126, 1577-84; (b) Gilbert, Y.; Deghorain, M.; Wang, L.; Xu, B.; Pollheimer, P. D.; Gruber, H. J.; Errington, J.; Hallet, B.; Haulot, X.; Verbelen, C.; Hols, P.; Dufrene, Y. F., Nano Lett. 2007, 7, 796-801.

40. Hinterdorfer, P.; Schilcher, K.; Baumgartner, W.; Gruber, H. J.; Schindler, H., Nanobiol. 1998, 4, 177-88.

41. Hinterdorfer, P.; Kienberger, F.; Raab, A.; Gruber, H. J.; Baumgartner, W.; Kada, G.; Riener, C.; Wielert-Badt, S.; Borken, C.; Schindler, H., Single Mol. 2000, 1, 99-103.

42. Giannotti, M. I.; Vancso, G. J., ChemPhysChem 2007.

43. (a) Baumgartner, W.; Hinterdorfer, P.; Schindler, H., Ultramicroscopy 2000, 82, 85-95; (b) Lévy, R.; Maaloum, M., Ultramicroscopy 2005, 102, 311-5; (c) Odorico, M.; Teulon, J. M.; Berthoumieu, O.; Chen, S. w. W.; Parot, P.; Pellequer, J. L., Ultramicroscopy 2007, 107, 887-94; (d) Marsico, A.; Labudde, D.; Sapra, T.; Muller, D. J.; Schroeder, M., Bioinformatics 2007, 23, e231-e6; (e) Fuhrmann, A.; Anselmetti, D.; Ros, R.; Getfert, S.; Reimann, P., Phys. Rev. E Stat. Nonlin. Soft Matter Phys. 2008, 77, 031912.

44. Sandal, M.; Benedetti, F.; Brucale, M.; Gomez-Casado, A.; Samori, B., Bioinformatics 2009, 25, 1428-30.

45. Bjornham, O.; Schedin, S., Eur Biophys J 2009, 38, 911-22. 
46. (a) Hummer, G.; Szabo, A., Biophys. J. 2003, 85, 5-15; (b) Dudko, O. K.; Hummer, G.; Szabo, A., Phys. Rev. Lett. 2006, 96, 108101.

47. Jarzynski, C., Phys. Rev. Lett. 1997, 78, 2690.

48. (a) Liphardt, J.; Dumont, S.; Smith, S. B.; Tinoco, I.; Bustamante, C., Science 2002, 296, 1832-5; (b) Harris, N. C.; Song, Y.; Kiang, C. H., Phys. Rev. Lett. 2007, 99, 068101; (c) Bizzarri, A. R.; Cannistraro, S., Phys. Chem. Chem. Phys. 2011, 13, 2738-43.

49. (a) Favre, M.; Sekatskii, S. K.; Dietler, G., Ultramicroscopy 2008, 108, 1135-9; (b) Guo, S.; Lad, N.; Ray, C.; Akhremitchev, B. B., Biophys. J. 2009, 96, 3412-22.

50. Fernandez, J. M.; Li, H., Science 2004, 303, 1674-8.

51. (a) Hinterdorfer, P.; Baumgartner, W.; Gruber, H. J.; Schilcher, K.; Schindler, H., Proc. Natl. Acad. Sci. U.S.A. 1996, 93, 3477-81; (b) Hinterdorfer, P.; Dufrene, Y. F., Nat Methods 2006, 3, 347-55.

52. (a) Raab, A.; Han, W.; Badt, D.; Smith-Gill, S. J.; Lindsay, S. M.; Schindler, H.; Hinterdorfer, P., Nat. Biotechnol. 1999, 17, 901-5; (b) Stroh, C. M.; Ebner, A.; Geretschlager, M.; Freudenthaler, G.; Kienberger, F.; Kamruzzahan, A. S.; Smith-Gill, S. J.; Gruber, H. J.; Hinterdorfer, P., Biophys. J. 2004, 87, 1981-90; (c) Bonanni, B.; Kamruzzahan, A. S.; Bizzarri, A. R.; Rankl, C.; Gruber, H. J.; Hinterdorfer, P.; Cannistraro, S., Biophys. J. 2005, 89, 2783-91; (d) Zhu, R.; Howorka, S.; Proll, J.; Kienberger, F.; Preiner, J.; Hesse, J.; Ebner, A.; Pastushenko, V. P.; Gruber, H. J.; Hinterdorfer, P., Nat. Nanotechnol. 2010, 5, 788-91.

53. Duman, M.; Pfleger, M.; Zhu, R.; Rankl, C.; Chtcheglova, L. A.; Neundlinger, I.; Bozna, B. L.; Mayer, B.; Salio, M.; Shepherd, D.; Polzella, P.; Moertelmaier, M.; Kada, G.; Ebner, A.; Dieudonne, M.; Schutz, G. J.; Cerundolo, V.; Kienberger, F.; Hinterdorfer, P., Nanotechnology 2010, 21, 115504.

54. (a) Hohng, S.; Zhou, R.; Nahas, M. K.; Yu, J.; Schulten, K.; Lilley, D. M. J.; Ha, T., Science 2007, 318, 279-83; (b) Murade, C. U.; Subramaniam, V.; Otto, C.; Bennink, M. L., Nucleic Acids Res. 2010, 38, 3423-31.

55. (a) Wiita, A. P.; Ainavarapu, S. R. K.; Huang, H. H.; Fernandez, J. M., Proc. Natl. Acad. Sci. U.S.A. 2006, 103, 7222-7; (b) Koti Ainavarapu, S. R.; Wiita, A. P.; Dougan, L.; Uggerud, E.; Fernandez, J. M., J. Am. Chem. Soc. 2008, 130, 6479-87; (c) Garcia-Manyes, S.; Liang, J.; Szoszkiewicz, R.; Kuo, T.-L.; Fernández, J. M., Nat. Chem. 2009, 1, 236-42.

56. Williams, J. M.; Han, T.; Beebe, T. P., Langmuir 1996, 12, 1291-5.

57. (a) Seifert, U., Phys. Rev. Lett. 2000, 84, 2750-3; (b) Williams, P. M., Anal. Chim. Acta 2003, 479, 107-15.

58. Sulchek, T.; Friddle, R. W.; Noy, A., Biophys. J. 2006, 90, 4686-91.

59. Tees, D. F. J.; Woodward, J. T.; Hammer, D. A., J. Chem. Phys. 2001, 114, 7483-96.

60. Ushakov, I. A.; Harrison, R. A., Wiley 1994. 
61. (a) Puchner, E. M.; Kufer, S. K.; Strackharn, M.; Stahl, S. W.; Gaub, H. E., Nano Lett. 2008, 8, 3692-5; (b) Kufer, S. K.; Puchner, E. M.; Gumpp, H.; Liedl, T.; Gaub, H. E., Science 2008, 319, 594-6.

62. (a) Carvalho, F. A.; Connell, S.; Miltenberger-Miltenyi, G.; Pereira, S. V.; Tavares, A.; Ariëns, R. A. S.; Santos, N. C., ACS Nano 2010, 4, 4609-20; (b) Maki, T.; Kidoaki, S.; Usui, K.; Suzuki, H.; Ito, M.; Ito, F.; Hayashizaki, Y.; Matsuda, T., Langmuir 2007, 23, 2668-73.

63. (a) Sarkar, A.; Caamano, S.; Fernandez, J. M., Biophys. J. 2007, 92, L368; (b) Verbelen, C.; Gruber, H. J.; Dufrene, Y. F., J. Mol. Recognit. 2007;

(c) Florin, E. L.; Moy, V. T.; Gaub, H. E., Science 1994, 264, 415-7; (d) Handa, H.; Gurczynski, S.; Jackson, M. P.; Mao, G., Langmuir 2010, 26, 12095-103.

64. Lo, Y. S.; Huefner, N. D.; Chan, W. S.; Stevens, F.; Harris, J. M.; Beebe, T. P., Langmuir 1999, 15, 1373-82.

65. Kudera, M.; Eschbaumer, C.; Gaub, H. E.; Schubert, U. S., Adv. Funct. Mater. 2003, 13, 615-20.

66. Ratto, T. V.; Rudd, R. E.; Langry, K. C.; Balhorn, R. L.; McElfresh, M. W., Langmuir 2006, 22, 1749-57.

67. Sulchek, T. A.; Friddle, R. W.; Langry, K.; Lau, E. Y.; Albrecht, H.; Ratto, T. V.; DeNardo, S. J.; Colvin, M. E.; Noy, A., Proc. Natl. Acad. Sci. U.S.A. 2005, 102, 16638-43.

68. Zhang, Y.; Yu, Y.; Jiang, Z.; Xu, H.; Wang, Z.; Zhang, X.; Oda, M.; Ishizuka, T.; Jiang, D.; Chi, L.; Fuchs, H., Langmuir 2009, 25, 6627-32.

69. Gu, C.; Kirkpatrick, A.; Ray, C.; Guo, S.; Akhremitchev, B. B., J. Phys. Chem. C 2008, 112, 5085-92.

70. (a) Guo, S.; Li, N.; Lad, N.; Desai, S.; Akhremitchev, B. B., J. Phys. Chem. C 2010, 114, 8755-65; (b) Guo, S.; Ray, C.; Kirkpatrick, A.; Lad, N.; Akhremitchev, B. B., Biophys. J. 2008, 95, 3964-76.

71. (a) Zou, S.; Schönherr, H.; Vancso, G. J., J. Am. Chem. Soc. 2005, 127, 11230-1; (b) Friddle, R. W.; Podsiadlo, P.; Artyukhin, A. B.; Noy, A., J. Phys. Chem. C 2008, 112, 4986-90. 


\section{Chapter 3}

\section{Charge-transfer complexes studied by dynamic force spectroscopy}

In this chapter the strength and kinetics of two charge-transfer complexes, naphtholmethylviologen and pyrene-methylviologen, are studied using dynamic force spectroscopy. The dissociation rates indicate an enhanced stability of the pyrenemethylviologen complex, which agrees with its higher thermodynamic stability compared to naphthol-methylviologen complex. 


\subsection{Introduction}

Non-covalent interactions, such as hydrogen bonds, $\pi-\pi$ stacking, electrostatic and hydrophobic interactions, are ubiquitous in biological systems. Their inherent reversibility makes them also interesting for technological applications such as stimulus-responsive surfaces and molecular recognition. One of these non-covalent interactions is the charge-transfer (CT) interaction. A CT complex is formed when a partial transfer of charge occurs between two molecules. The molecule providing the electron (donor) and the receiver of the electron (acceptor) experience an electrostatic attraction that holds them together. The electron responsible of the $\mathrm{CT}$ interaction occupies an excited state, which is often evidenced by the appearance of characteristic absorption bands in the visible spectra. The CT interaction appears in many biological systems, ${ }^{1}$ in particular it stabilizes molecules that intercalate between DNA bases, which can be used for cancer therapy. ${ }^{2} \mathrm{CT}$ interactions have also been exploited in the fabrication of layer-by-layer films. ${ }^{3}$ The acceptor, methylviologen (mv, N,N'dimethyl-4,4'-bipyridinium), is a dication and has been extensively used in the study of CT complexes. ${ }^{4}$ The complexation of $\mathrm{mv}$ with molecules containing donor moieties has been applied to create supramolecular crystals ${ }^{5}$ and amphiphiles $^{6}$ as well as in phototherapy. ${ }^{7}$ In particular, host-stabilized CT complexes of $\mathrm{mv}$ have been used as building blocks of redox-switchable supramolecular surfaces ${ }^{8}$ and polymers. ${ }^{9}$

Single-molecule force spectroscopy (SMFS) has been used to study weak interactions quantitatively, both of biological and synthetic origin. ${ }^{10}$ In particular, the atomic force microscope (AFM) can be easily adapted to perform this type of measurements. The kinetic properties of the studied complexes reveal themselves as a dependence of the force required to rupture the complex with the speed at which the complex is being pulled at. ${ }^{11}$ Thus, force spectroscopy experiments are often performed covering a range of pulling speeds in what has been named dynamic force spectroscopy (DFS). The strength of CT interactions has been examined using force spectroscopy before. ${ }^{12}$ The interaction between tetramethylphenylenediamine (TMPD) and tetracyanoquinodimethane (TCNQ) was reported to rupture at $70 \mathrm{pN},{ }^{12 a}$ and polymers decorated with poly(9-vinylcarbazole) (PVK) were found to desorb from monolayers presenting dinitrobenzamide (DBA) and nitrobenzamide (NBA) groups at forces of 28 and $18 \mathrm{pN}$, respectively. ${ }^{12 c}$ However, many of the 
experiments were not conducted at the single molecule level, ${ }^{12 a, b}$ or did not study the interaction over a dynamic range. ${ }^{12 a}$

Here, the kinetic properties of two CT complexes, naphthol-methylviologen (nph-mv, see Scheme 3.1) and pyrene- methylviologen (pyr-mv) are studied using DFS.

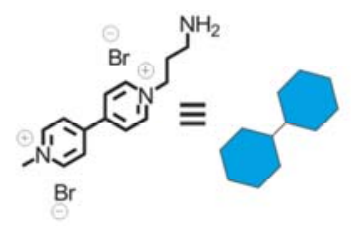

$m v$
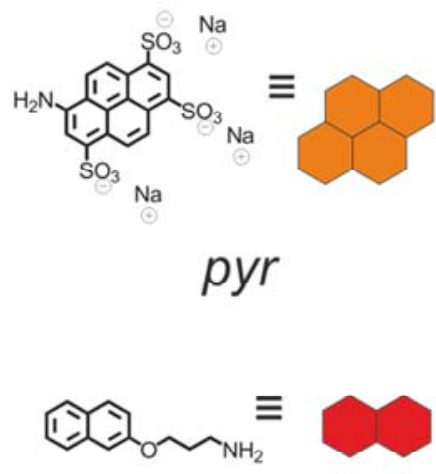

nph

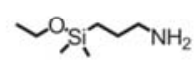

APDES

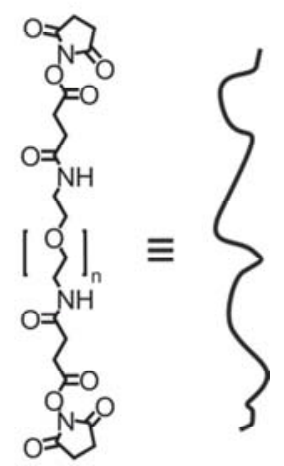

PEG 3000

Scheme 3.1 Compounds used in this chapter.

\subsection{Results and discussion}

\subsubsection{Double-tethered DFS}

In order to perform a DFS experiment the studied complex must be ruptured repeatedly in a controlled manner. ${ }^{10 a}$ The complementary moieties are secured to the surface of an AFM tip and a supporting substrate. The distance between the base of the AFM cantilever and the substrate is varied by means of a piezoelectric crystal. In the starting point the two surfaces are away from each other at a distance on the order of hundreds to thousands of $n m$, so the AFM 
cantilever is relaxed. This distance is reduced until the tip makes contact to the surface, and further reduced until a predetermined deflection, which is measured using a laser beam reflecting on the cantilever, is achieved. This part of the cycle is called the approach. The system is kept in this situation for a certain amount of time (dwell time, usually 0.1-1 s) to allow the formation of the complexes. Then, the distance between the base of the cantilever and the substrate is increased at a constant speed, the so-called retraction part of the cycle. In the initial phase of the retraction the cantilever will relax, followed by a deflection on the opposite direction if an interaction occurs between the tip surface and the substrate. When the energy stored in the negatively deflected cantilever is enough to break the interaction a sudden relaxation will be detected and the cantilever will travel undeflected until the starting point. The cantilever spring constant can be calibrated using several methods to translate these deflection values into force values. ${ }^{13}$ A plot of the measured force versus the imposed distance (a force-distance curve) can be constructed using this information. However, it is unlikely that the interaction between the tip surface and the substrate measured in such way originates exclusively from the interaction between the studied moieties. The strongest interaction when doing this experiment in ambient conditions is the capillary force produced by the water meniscus that forms at the point of contact, which is the reason why DFS experiments are carried out inside a liquid medium in most occasions. Several other interactions, such as Van der Waals, electrostatic or hydrophobic forces can contribute to the attractive interaction between the tip and the substrate. Long polymeric linkers, in particular polyethylene glycol (PEG), ${ }^{14}$ are usually employed to connect the tip surface and one of the moieties in order to separate unspecific contributions from the studied interaction. ${ }^{15}$ Thus, the typically short-ranged unspecific interactions are broken before any stress is exerted on the complex (Figure 3.1). Moreover, in many cases the formation of the complex requires a certain degree of freedom that can only be achieved when introducing a flexible linker instead of a direct attachment to the surface. The characteristic non-linear elasticity of polymeric linkers appears on the force-distance curve as a curved line, compared to the constant slope preceding the rupture of non-specific surface interactions. The slope of the points just prior to the rupture (in $\mathrm{pN} / \mathrm{nm}$ ) can be multiplied by the externally imposed retraction speed (in $\mathrm{nm} / \mathrm{s}$ ) in order to obtain the instantaneous loading rate (how fast the force was applied) that was exerted on the complex (in $\mathrm{pN} / \mathrm{s}$ ). 


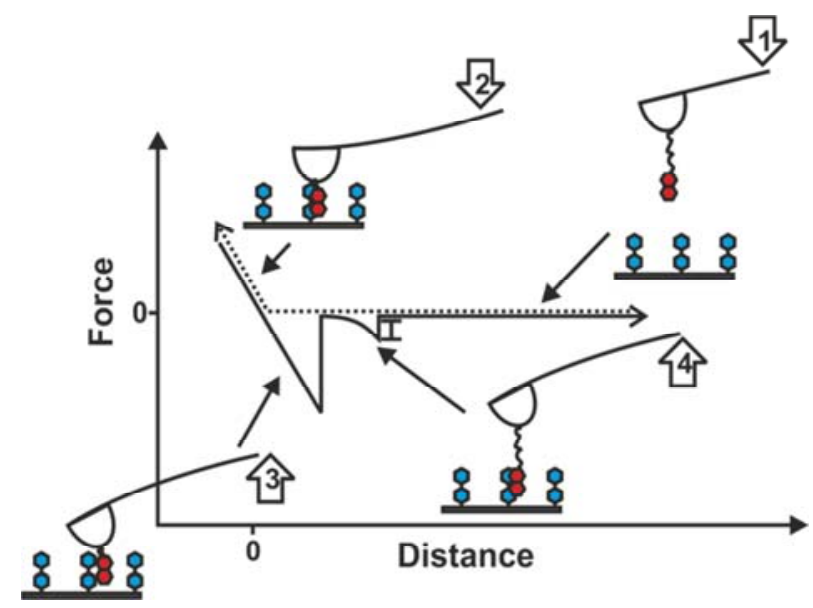

Figure 3.1 Force-distance curve using a tethered moiety. During the approach phase the cantilever is relaxed (1) until the tip snaps into contact with the substrate and is deflected (2). During the retraction a negative force (unspecific interaction) is detected with a constant slope while the tip and substrate are in contact (3). After this interaction is broken, a non-linear region appears as the polymer chain is stretched (4).

It has been shown for several systems that the force required to break a bond depends on the loading rate, which is the reason why the rupture force should be measured at different retraction speeds. ${ }^{11,16}$ Occasionally, saw-tooth patterns are observed when multiple complexes tethered by chains of different length are successively ruptured. In our study we only analyzed the last rupture of each force-distance curve to ensure that the observed instantaneous loading rate is not being shared with other complexes. Polymer models such as the worm-like chain (WLC) or the freely-jointed chain (FJC) can be fitted to the nonlinear segments to discriminate whether more than one chain or a single chain was being pulled. This ensures that the measured force corresponds to the rupture force of a single complex, and not to two or more complexes being ruptured at the same instant. Since the thermal energy at room temperature is comparable to the energy of CT interactions, the rupture will be a thermally activated process. Thus, the rupture force will vary several $\mathrm{pN}$ depending on the microscopic fluctuations of the system, and several ruptures must be measured in order to obtain a reliable most probable rupture force. 
A
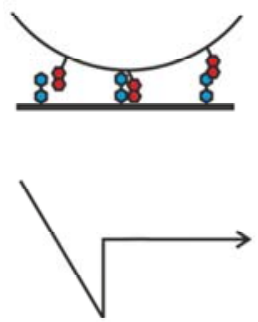

B
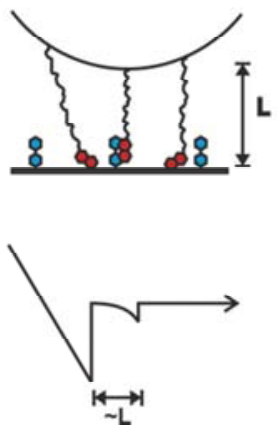
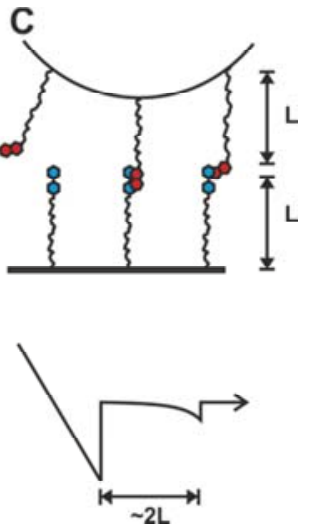

Figure 3.2 Attachment strategies and associated characteristic forcedistance curves. A) Direct linking does not allow the estimation of the number of complexes and the rupture force can be affected by unspecific contributions. B) Tethering one of the moieties resolves the rupture from the unspecific tip-substrate interaction, but substratemoiety interactions are still possibly present. C) Double-tethering ensures that ruptures occurring when the tip-substrate distance is approx. two tether lengths originate from specific interactions.

However this scheme does not rule out possible interactions between the tethered moiety and the opposite substrate. The use of the so-called doubletether approach (Figure 3.2), in which a linker is introduced to connect each of the complementary moieties to their corresponding surface, is used to solve this problem. ${ }^{17}$ Only ruptures that occur when the tip and the substrate are at distances approximately two times the length of the employed linker are in this approach regarded as specific.

\subsubsection{Naphthol - methylviologen complex}

Following the double-tether strategy, we used a commercially available bis- $\mathrm{N}$ hydroxysuccinimide (NHS) polyethylene glycol crosslinker (PEG, $M_{w} 3000$ ) to connect amine-functionalized $\mathrm{nph}$ and $\mathrm{mv}$ derivatives to amine-functionalized AFM cantilevers and silicon substrates, respectively. Several hundreds of approach-retraction cycles were performed at different retraction speeds. The resulting force-distance curves for each retraction speed were examined using a force spectroscopy analysis tool, ${ }^{18}$ and only the ones showing non-linear features corresponding to a single polymer chain were selected. Each selected 
curve produced a set of data consisting of values for the rupture force, the instantaneous loading rate and the contour length of the stretched polymer. This contour length is the combined length of two polymer chains in the case of a double-tethered rupture, or just one chain in the case of an unspecific rupture. Thus, the contour lengths were used to select forces originating from ruptures of the CT complex. The average contour length of a PEG chain of 3000 $\mathrm{g} / \mathrm{mol}$ is $25 \mathrm{~nm}$, however, the compound is polydisperse and, when looking at the last rupture event of the curves, these events originate from the longer chains on the tip. We have used the same compound in other studies where only one of the moieties forming the complex was tethered, the other being directly attached to a substrate. In all of these experiments we observed that more than $95 \%$ of the chains corresponding to the last rupture were shorter than $60 \mathrm{~nm}$ (see experimental section of Chapter 5). Based on this observation, we only considered for further analysis the ruptures corresponding to chains of larger contour lengths, which we attribute to double-tethered interacting pairs.

The resulting data after this filtering procedure was used to construct kernel density estimations of rupture force and instantaneous loading rate at each retracting speed. The most probable force, loading rate and their uncertainties were extracted from these density plots. In some cases a long tail of the distribution towards higher forces was observed (Figure 3.3). This tail has been attributed to diverse causes such as heterogeneity of chemical bonds ${ }^{19}$ or simultaneous ruptures of more than one complex. ${ }^{20}$

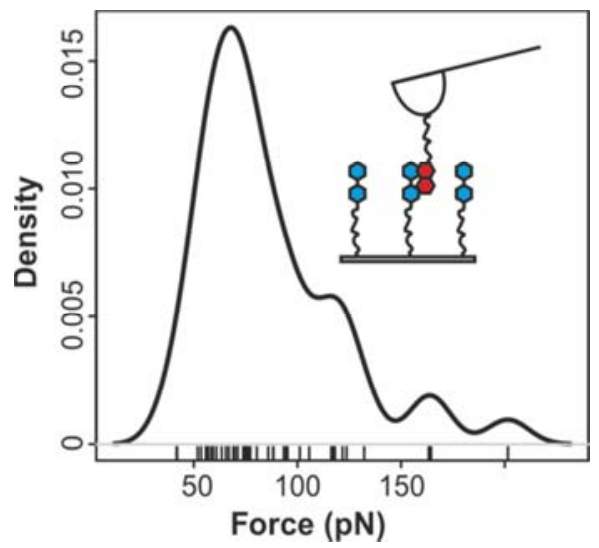

Figure 3.3 Density of probability for the rupture force of nph-mv probed at $598 \mathrm{~nm} / \mathrm{s}$. The ticks on the horizontal axis indicate individual measurements. 
A plot of the most probable rupture force versus the most probable loading rate at each retracting speed is shown in Figure 3.4. The most probable rupture force, $f^{*}$, increases with the loading rate, $\rho$. Such behavior is characteristic of complexes being pulled out of equilibrium. The relationship between these two quantities was first described in the work of Evans and Ritchie. ${ }^{11}$

$f^{*}(\rho)=\frac{k_{B} T}{x_{\beta}} \ln \left(\frac{\rho x_{\beta}}{k_{B} T k_{o f f}^{*}}\right)$

In this expression $k_{B} T$ is the thermal energy, $x_{B}$ is the width of the potential barrier of the interaction and $k^{*}$ off is the dissociation rate at zero force (the intrinsic off-rate of the complex). The solution of this equation appears as a straight line in a semilogarithmic plot. Although we could fit the data to this model (dashed line in Figure 3.4), we found that a better fit was obtained ignoring the point at the lowest loading rate (solid line in Figure 3.4). The results from the fit of the high loading rate regime suggest a potential width $x_{6}$ of $0.16 \pm 0.03 \mathrm{~nm}$ and a dissociation rate constant $k_{\text {off }}^{*}$ of $14 \mathrm{~s}^{-1}$ (confidence interval 8-23 $\left.\mathrm{s}^{-1}\right)$.

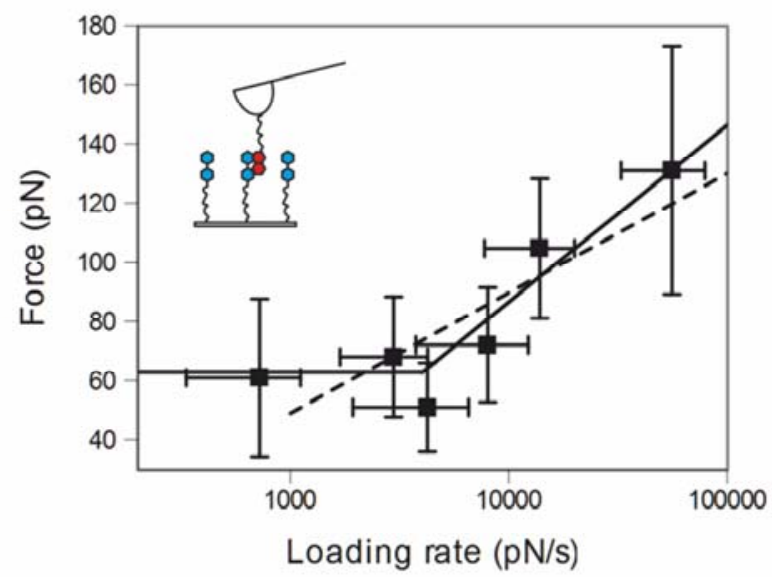

Figure 3.4 Results from DFS performed on the nph-mv CT complex. The lines are fits to Evans model using all points (dashed) or all but the first point and a constant force value at low loading rates (solid). 


\subsubsection{Pyrene-methylviologen complex}

A similar surface functionalization scheme was used for substituting the aminofunctionalized nph derivative by amino-functionalized pyrene, (pyr). The resulting force-distance curves were analyzed and filtered as described above. A plot showing the density of probability for the rupture force of pyr-mv is shown in Figure 3.5.

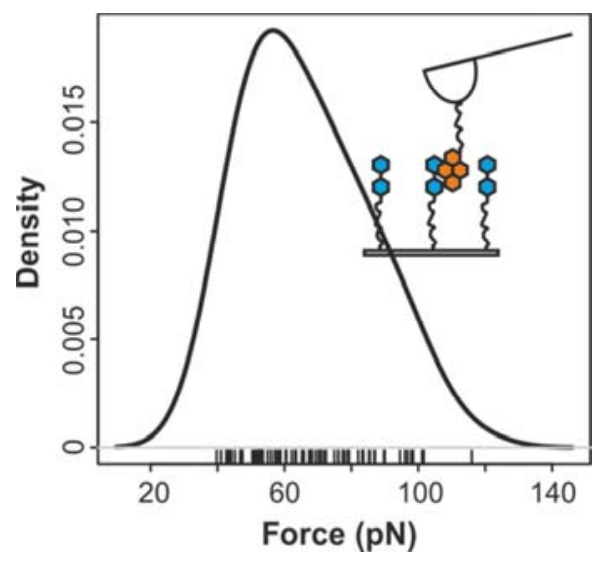

Figure 3.5 Density of probability for the rupture force of pyr-mv probed at $581 \mathrm{~nm} / \mathrm{s}$. The ticks in the horizontal axis indicate individual measurements.

The most probable rupture force versus loading rate values is presented in Figure 3.6. In this case ignoring the lowest loading rate point, did not improve the fit. The fit to the Evans model of all data points suggests a potential width $x_{6}$ of $0.4 \pm 0.1 \mathrm{~nm}$ and a dissociation rate constant $k^{*}$ off of $2 \mathrm{~s}^{-1}$ (confidence interval $\left.0.3-13 s^{-1}\right)$. 


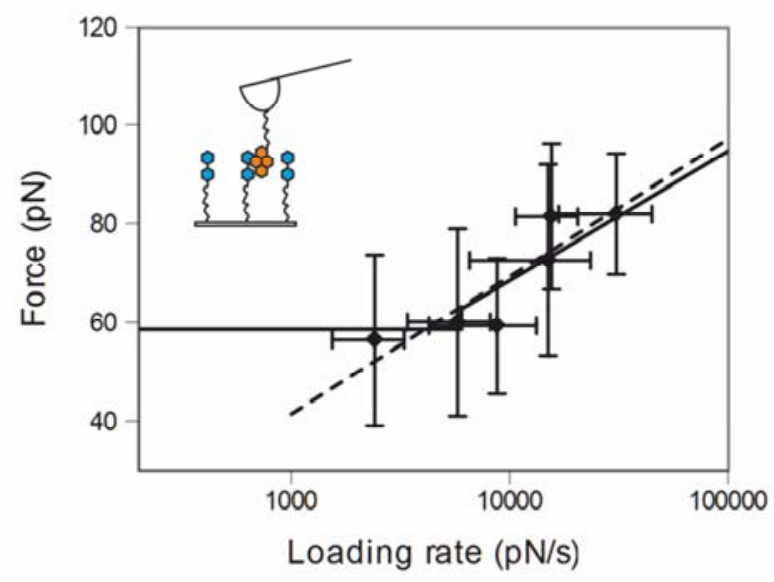

Figure 3.6 Results from DFS performed on the pyr-mv CT complex. The lines are fits to Evans model using all points (dashed) or all but the first point and a constant force value at low loading rates (solid).

\subsubsection{Comparing nph-mv and pyr-mv complexes}

Comparing the characteristic potential widths and dissociation rate constants of the two CT complexes studied here we observe differences that agree qualitatively with our expectations. First, the potential width is appreciably larger in the case of the pyr-mv complex $(0.4 \pm 0.1 \mathrm{~nm})$ when compared to the nph-mv complex $(0.16 \pm 0.03 \mathrm{~nm})$. This can be correlated to the molecular structure of pyrene and naphthol, the larger size of the surface of the pyrene molecule will allow a bigger relative displacement of the two molecules until the interaction is broken. Secondly, the lower dissociation rate constant of the pyr-mv complex $\left(2 \mathrm{~s}^{-1}\right)$ indicates that this complex is more stable than nph-mv $\left(14 \mathrm{~s}^{-1}\right)$, which is compatible with the higher equilibrium constant of the pyr-mv complex. ${ }^{6,21}$ Moreover, the force versus loading rate data of the nph-mv system are preferentially fitted to two different straight lines. The occurrence of different regimes of force versus loading rate has been reported before for other supramolecular complexes and interpreted as the emergence of inner barriers of the potential. ${ }^{22}$ However, at low loading rates the dependence of the rupture force on the loading rate seems to be null. This behavior has also been reported for fast rebinding $\pi-\pi$ complexes probed at near-equilibrium, such as the interaction between pyrene and graphite. ${ }^{23} \mathrm{~A}$ similar transition has been observed by increasing the temperature in DFS experiments using quadruple hydrogen-bonded dimers. ${ }^{24}$ This suggests that the nph-mv complex 
is being pulled close to thermodynamic equilibrium at low loading rates (under approx. $\left.4000 \mathrm{pNs}^{-1}\right)$. The equilibrium rupture force for mv-nph ruptures $(63 \pm$ $20 \mathrm{pN}$ ) is much larger than the equilibrium rupture force observed for PVK-NBA $(18 \mathrm{pN})$ and PVK-DBA (28 pN) complexes at loading rates ranging from 15000 to $75000 \mathrm{pNs}^{-1}{ }^{12 \mathrm{c}}$ However, it compares well with the TMPD-TCNQ rupture force $(70 \pm 15 \mathrm{pN})$ at a comparable loading rate $\left(4500 \mathrm{pNs}^{-1}\right){ }^{12 a}$ Unfortunately, the TMPD-TCNQ complex was not studied at different loading rates, which would allow a comparison of the possible dynamic behavior. On the other hand, the pyr-mv complex appear to be pulled out-of-equilibrium at all explored loading rates, which agrees with the slower dissociation kinetics observed in this case.

\subsection{Conclusions}

The rupture forces of two different CT complexes were measured at the single molecule level over a range of retraction speeds. In the case of the nph-mv complex, a transition from near-equilibrium to a loading-rate dependent behavior was observed with increasing loading rate, while the pyr-mv complex was probed far from equilibrium at all the applied loading rates. The dissociation rate constants obtained from fits of a theoretical model to the data confirm an enhanced stability of the pyr-mv complex when compared to the nph-mv complex. The differences in potential width and dissociation rate constant appear to be qualitatively in concert with the differences in molecular structure of the donors. This study adds to the understanding of the kinetic behavior of CT interactions, which can be useful for the design of DNA intercalating drugs with improved stability and presumably will influence the reversibility of structures relying on this type of interaction such as layer-bylayer assemblies.

\subsection{Experimental}

\section{Materials}

All solvents were purchased from commercial sources and used as received unless explicitly noted. Aminopropyldimethylethoxysilane (APDES), PEG 3000 (succinimidyl-[N-methyl]- (NHS)-terminated homobifunctional PEG, $M_{W}$ 3000) 
and 8-aminopyrene-1,3,6-trisulfonic acid trisodium salt (pyr) were purchased from $A B C R$ and Sigma-Aldrich. Water was purified and deionized (MQ water, resistivity of $18.2 \mathrm{M} \Omega \mathrm{cm}^{-1}$ ) .

\section{3-(2-Naphthyl)-oxy-aminopropane (nph)}

A mixture of naphthol (1) (1.00 g, $6.93 \mathrm{mmol}), \mathrm{N}$-(3-bromopropyl)-phthalimide (2) (1.85 g, $6.93 \mathrm{mmol})$, dry potassium carbonate $(1.15 \mathrm{~g}, 6.93 \mathrm{mmol})$ and 18 crown- 6 ether in $60 \mathrm{ml}$ of acetone was refluxed for $12 \mathrm{~h}$. After cooling the mixture to room temperature, the volatiles were removed under reduced pressure. The reaction mixture was extracted with $\mathrm{CH}_{2} \mathrm{Cl}_{2}$. The organic layer was washed with water and dried over anhydrous $\mathrm{MgSO}_{4}$. The solvent was evaporated to obtain $\mathbf{3}$ as a white solid.

Then, a mixture of $3(0.50 \mathrm{~g}, 1.51 \mathrm{mmol})$, hydrazine $(0.22 \mathrm{ml}, 1.51 \mathrm{mmol})$ in 20 $\mathrm{ml}$ of ethanol was refluxed for $2 \mathrm{~h}$. The mixture was then acidified to $\mathrm{pH} 1$ with a solution of $1 \mathrm{M} \mathrm{HCl}$ and refluxed for $1.5 \mathrm{~h}$. After this time, the $\mathrm{pH}$ was adjusted with a solution of $1 \mathrm{M} \mathrm{NaOH}$ and the mixture was extracted with $\mathrm{CHCl}_{3}$ and dried over anhydrous $\mathrm{MgSO}_{4}$. The volatiles were removed under reduced pressure and a brownish solid was obtained. To this brownish solid $\mathrm{CHCl}_{3}$ was added and the white precipitated formed was filtered through celite. After removal of the solvent, 4 was obtained as a brownish solid. Yield: $0.15 \mathrm{~g}, 49 \%$. 1H NMR (ppm): $\delta=7.71-7.64(\mathrm{~m}, \mathrm{CH}, 3 \mathrm{H}), 7.39-7.26(\mathrm{~m}, \mathrm{CH}, 2 \mathrm{H}), 7.09-7.05(\mathrm{~m}$, $\mathrm{CH}, 2 \mathrm{H}), 4.11\left(\mathrm{t}, \mathrm{CH}_{2},{ }^{3} \mathrm{~J}(\mathrm{H}, \mathrm{H})=6.0 \mathrm{~Hz}, 2 \mathrm{H}\right), 3.62(\mathrm{br} \mathrm{s}), 2.90\left(\mathrm{t}, \mathrm{CH}_{2},{ }^{3} \mathrm{~J}(\mathrm{H}, \mathrm{H})=6.0\right.$ $\mathrm{Hz}, 2 \mathrm{H}), 1.94\left(\mathrm{~m}, \mathrm{CH}_{2},{ }^{3} \mathrm{~J}(\mathrm{H}, \mathrm{H})=6.0 \mathrm{~Hz}, 2 \mathrm{H}\right)$

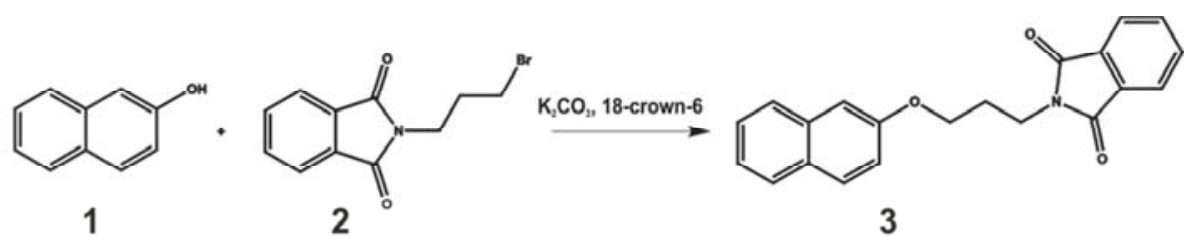

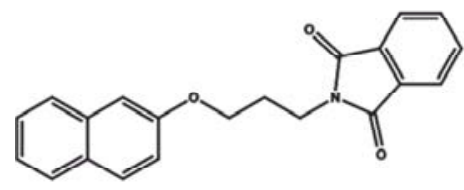

3

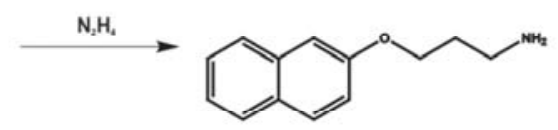

4

Scheme 3.2 (Note that compound 4 refers to $\mathrm{nph}$ in Scheme 3.1). 


\section{Substrate and AFM cantilever functionalization}

Silicon substrates (about $1 \mathrm{~cm}^{2}$ ) were repeatedly washed in dichloromethane (DCM) and methanol. The substrates were oxidized in oxygen plasma for $20 \mathrm{~s}$ and then immersed into a (1:200) solution of APDES in distilled dry toluene for $1 \mathrm{~min}$. Then, they were rinsed with copious amounts of DCM. The amineterminated substrates were incubated for $2 \mathrm{~h}$ in a 200:1 DCM/ N,Ndiisopropylethylamine (DIPEA) solution with PEG 3000 crosslinker (NHS-PEGNHS, $5 \mathrm{mg} / \mathrm{ml}, \sim 1 \mathrm{mM}$ ) and rinsed with DCM. The AFM cantilevers (MLCT, Bruker) were treated following the same procedure. The mv-terminated substrates were prepared incubating the substrates for $2 \mathrm{~h}$ in a solution of amino-terminated $\mathrm{mv}(1 \mathrm{mM})$ in 200:1 MQ water/DIPEA and rinsed with water. Finally nph- or pyr-terminated tips were obtained by immersing the AFM cantilevers for $2 \mathrm{~h}$ in a solution of amino-terminated $\mathrm{nph}$ or pyr (1 mM) in 100:1 DCM/DIPEA or 200:1 MQ water/DIPEA, respectively, and rinsing with the corresponding solvent. The substrates and tips were kept in ultrapure water until they were used.

\section{AFM force spectroscopy measurements}

All force measurements were performed with a commercial Multimode Picoforce SPM (Veeco, Digital Instruments, USA) using a liquid cell (Veeco) and saline aqueous solutions ( $10 \mathrm{mM} \mathrm{NaCl}$ in $\mathrm{MQ}$ water). The spring constants of the cantilevers were calibrated using the built-in thermal tuning software. Force-distance curves were acquired by approaching and retracting the tip at speeds ranging $500-3000 \mathrm{~nm} / \mathrm{s}$ and a dwell time of $0.1 \mathrm{~s}$. The tip was laterally displaced over the substrate between approach-retraction cycles, covering 1 $\mu \mathrm{m}^{2}$, and the maximum force applied to the surface was kept under $500 \mathrm{pN}$. Approx. 5000 curves per cantilever could be obtained, limited by the appearance of high adhesion between the tip and surface and a large decrease in the chance of observing polymer stretching. This we attribute to deterioration of the monolayers due to the repeated contact or adsorption of contaminants on the tip.

\section{Data analysis}

We selected relevant force curves using our own plug-in script developed for Hooke. ${ }^{18}$ Each curve was examined for sudden changes in force (rupture events), then the data prior to the last rupture (the unbinding event happening farthest away from the surface) were fitted using a modified FJC model with a 
fixed Kuhn length of $7 \AA^{25}$ and the contour length was the fit parameter, and a WLC model where both the persistence length and the contour length were fit parameters. Rupture events were rejected or kept for further analysis based on the quality of the fit, which was assessed visually by comparing both fits, and numerically by comparing the fitted persistence length to the expected value of 3.5-3.8 $\AA$, in the case of WLC fits. In the case of the FJC fits, the averaged force difference from each data point to the corresponding fitted force was compared with the standard deviation of the measured force in the noncontact area. If the ratio of these two parameters is close to 1 , the difference between the fit and data can be explained by thermal noise. Pairs of rupture force and instantaneous loading rate were obtained from the valid events and an estimation of the rupture force and loading rate probability densities was obtained with the help of the statistical package $\mathrm{R}^{26}$ by using kernel density estimation with Epanechnikov kernels and a fixed bandwidth of $10 \mathrm{pN}$ in the case of force or an automatically selected bandwidth (Sheather and Jones algorithm $)^{27}$ in the case of loading rate. The resulting densities are shown in Figure 3.7 and Figure 3.8.
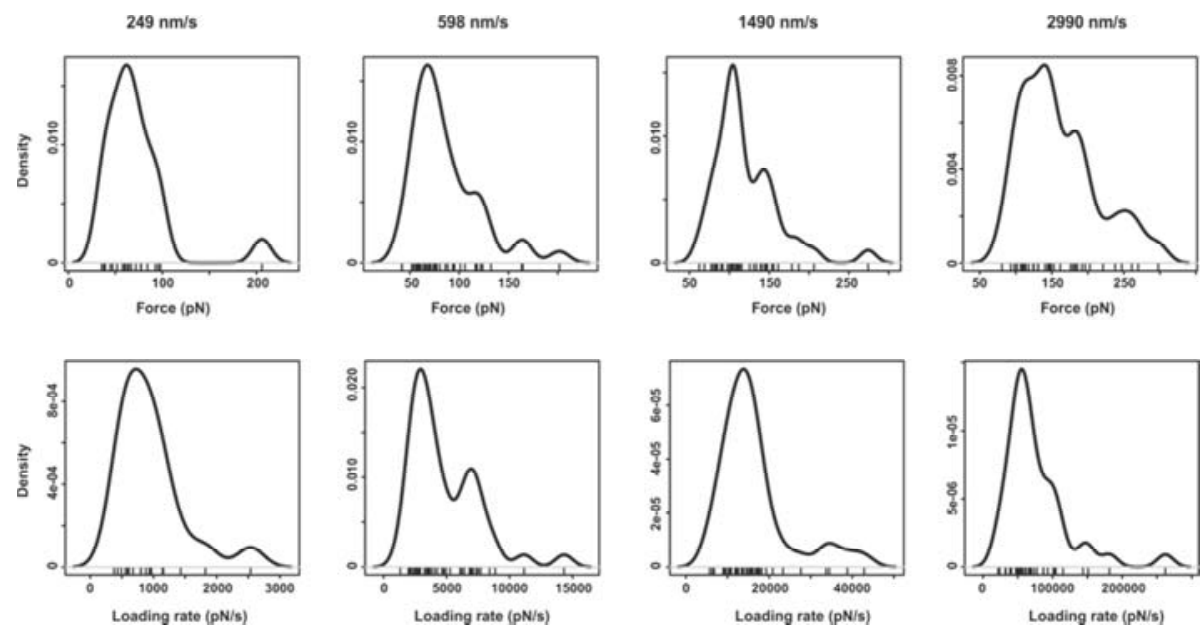

Figure 3.7 DFS results of the nph-mv CT complex. Density of probability for rupture force (top row) and instantaneous loading rate (bottom row) at different retraction speeds (columns). The ticks at the horizontal axis indicate individual measurements. 

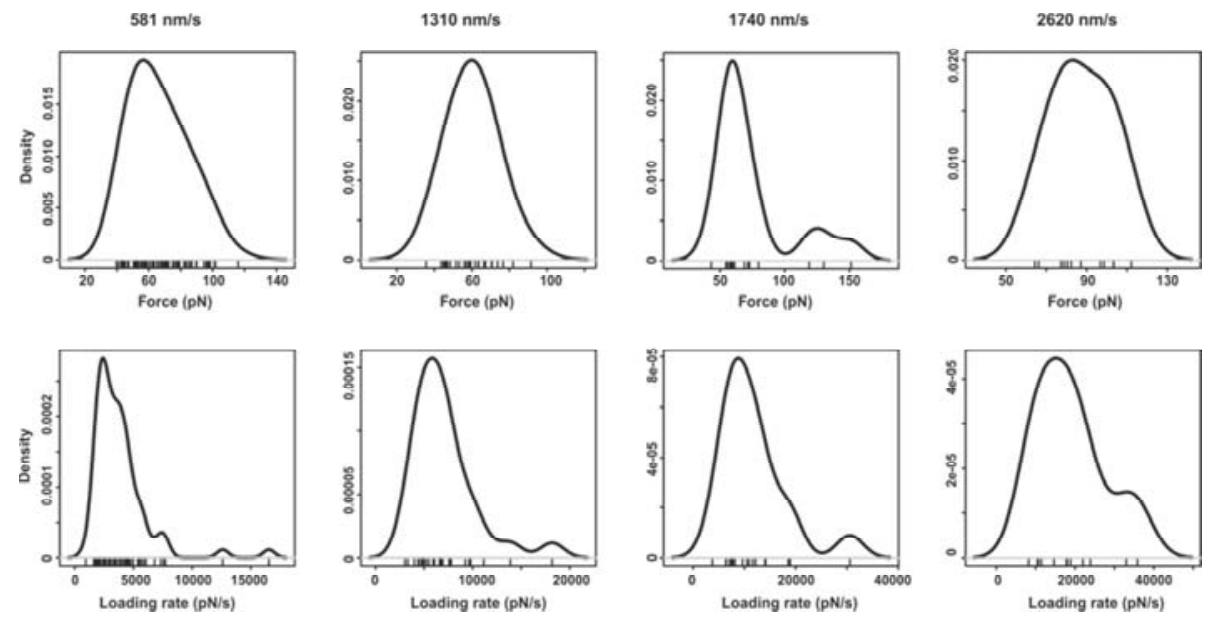

Figure 3.8 DFS results of the pyr-mv CT complex. Density of probability for rupture force (top row) and instantaneous loading rate (bottom row) at different retraction speeds (columns). The ticks at the horizontal axis indicate individual measurements.

\subsection{Acknowledgements}

We thank Dr. Arántzazu González Campo for the synthesis of the nph compound used in this study. Prof. Xi Zhang and Dr. Yiheng Zhang are acknowledged for fruitful discussions about the force spectroscopy experiments.

\subsection{References and notes}

1. (a) Shifrin, S., Ann. NY Acad. Sci. 1969, 158, 148-60; (b) Gutmann, F.; Johnson, C.; Keyzer, H.; Molnar, J., Charge Transfer Complexes in Biological Systems. CRC Press: 1997.

2. Liao, L. B.; Zhou, H. Y.; Xiao, X. M., J. Mol. Struct. 2005, 749, 108-13.

3. Shimazaki, Y.; Mitsuishi, M.; Ito, S.; Yamamoto, M., Langmuir 1997, 13, 1385-7.

4. (a) Ju, Z.-F.; Yao, Q.-X.; Wu, W.; Zhang, J., Dalton Trans. 2008, 355-62;

(b) Prasad, D. R.; Hoffman, M. Z., J. Phys. Chem. 1984, 88, 5660-5; (c) Nakahara, A.; Wang, J. H., J. Phys. Chem. 1963, 67, 496-8; (d) Monk, P. M. S.; Hodgkinson, N. M., Electrochim. Acta 1998, 43, 245-55; (e) Atherton, S. J.; Hubig, S. M.; Callan, T. J.; Duncanson, J. A.; Snowden, P. T.; Rodgers, M. A. J., J. Phys. Chem. 1987, 91, 3137-40. 
5. Kinuta, T.; Kise, Y.; Kamon, K.; Tajima, N.; Sato, T.; Kuroda, R.; Matsubara, Y.; Imai, Y., Tetrahedron Lett. 2009, 50, 5786-9.

6. Wang, C.; Guo, Y.; Wang, Y.; Xu, H.; Wang, R.; Zhang, X., Angew. Chem. Int. Ed. 2009, 48, 8962-5.

7. Hariharan, M.; Joseph, J.; Ramaiah, D., J. Phys. Chem. B 2006, 110, 24678-86.

8. Kang, J.-K.; Hwang, I.; Ko, Y. H.; Jeon, W. S.; Kim, H.-J.; Kim, K., Supramol. Chem. 2008, 20, 149 - 55.

9. Rauwald, U.; Scherman, O. A., Angew. Chem. Int. Ed. 2008, 47, 3950-3.

10. (a) Bizzarri, A. R.; Cannistraro, S., Chem. Soc. Rev. 2010, 39, 734-49; (b) Carvalho, F. A.; Connell, S.; Miltenberger-Miltenyi, G.; Pereira, S. V.; Tavares, A.; Ariëns, R. A. S.; Santos, N. C., ACS Nano 2010, 4, 4609-20; (c) Embrechts, A.; Schönherr, H.; Vancso, G. J., J. Phys. Chem. B 2008, 112, 7359-62.

11. Evans, E.; Ritchie, K., Biophys. J. 1997, 72, 1541-55.

12. (a) Skulason, H.; Frisbie, C. D., J. Am. Chem. Soc. 2002, 124, 15125-33;

(b) Gil, R.; Guillerez, M. G.; Poulin, J. C.; Schulz, E., Langmuir 2007, 23, 542-8; (c) Yu, Y.; Yao, Y.; Wang, L.; Li, Z., Langmuir 2010, 26, 3275-9.

13. (a) Hutter, J. L.; Bechhoefer, J., Rev. Sci. Instrum. 1993, 64, 1868-73; (b) Sader, J. E.; Chon, J. W. M.; Mulvaney, P., Rev. Sci. Instrum. 1999, 70, 3967-9.

14. Hinterdorfer, P.; Kienberger, F.; Raab, A.; Gruber, H. J.; Baumgartner, W.; Kada, G.; Riener, C.; Wielert-Badt, S.; Borken, C.; Schindler, H., Single Mol. 2000, 1, 99-103.

15. Christian, K. R.; Borken, C.; Kienberger, K.; Schindler, H.; Hinterdorfer, P.; Gruber, H. J., Single Mol. 2001, 2, 127-8.

16. Evans, E., Annu. Rev. Biophys. Biomol. Struct. 2001, 30, 105-28.

17. Ratto, T. V.; Langry, K. C.; Rudd, R. E.; Balhorn, R. L.; Allen, M. J.; McElfresh, M. W., Biophys. J. 2004, 86, 2430-7.

18. Sandal, M.; Benedetti, F.; Brucale, M.; Gomez-Casado, A.; Samori, B., Bioinformatics 2009, 25, 1428-30.

19. Raible, M.; Evstigneev, M.; Bartels, F. W.; Eckel, R.; Nguyen-Duong, M.; Merkel, R.; Ros, R.; Anselmetti, D.; Reimann, P., Biophys. J. 2006, 90, 3851-64.

20. (a) Gu, C.; Kirkpatrick, A.; Ray, C.; Guo, S.; Akhremitchev, B. B., J. Phys. Chem. C 2008, 112, 5085-92; (b) Guo, S.; Ray, C.; Kirkpatrick, A.; Lad, N.; Akhremitchev, B. B., Biophys. J. 2008, 95, 3964-76.

21. (a) Hsiao, J.-S.; Eckert, A. R.; Webber, S. E., J. Phys. Chem. 1994, 98, 12032-9; (b) Jung Hwang, H.; Kyoung Lee, S.; Lee, S.; Woo Park, J., J. Chem. Soc. Perkin Trans. 2 1999, 1081-6.

22. (a) Evans, E., Biophys. Chem. 1999, 82, 83-97; (b) Odorico, M.; Teulon, J. M.; Bessou, T.; Vidaud, C.; Bellanger, L.; Chen, S. W.; Quemeneur, E.; 
Parot, P.; Pellequer, J. L., Biophys. J. 2007, 93, 645-54; (c) Merkel, R.; Nassoy, P.; Leung, A.; Ritchie, K.; Evans, E., Nature 1999, 397, 50-3.

23. Zhang, Y.; Liu, C.; Shi, W.; Wang, Z.; Dai, L.; Zhang, X., Langmuir 2007, 23, 7911-5.

24. Zou, S.; Schönherr, H.; Vancso, G. J., J. Am. Chem. Soc. 2005, 127, 11230-1.

25. Oesterhelt, F.; Rief, M.; Gaub, H. E., New J. Phys. 1999, 1, 6.1-11.

26. RDC-Team, http://www.R-project.org 2008.

27. Sheather, S. J.; Jones, M. C., J. Roy. Stat. Soc. B Met. 1991, 683-90. 



\section{Chapter 4}

\section{Recognition properties of cucurbit[7]uril self-assembled monolayers studied with force spectroscopy}

In this chapter the recognition properties of self-assembled monolayers of cucurbit[7]uril on gold are studied using dynamic force spectroscopy. These monolayers are not optimally packed, as exposed areas of the underlying substrate were found interacting unspecifically with guest molecules. Single molecule force spectroscopy was used to discriminate and quantify specific and unspecific interactions of adamantane guests with this type of monolayers. 


\subsection{Introduction}

Cucurbit[n]urils ( $\mathrm{CB}[\mathrm{n}])$ are a family of barrel-shaped molecules that received their name for their resemblance to a pumpkin. ${ }^{1}$ They consist of $n$ repeating glycoluril units forming a macrocycle with a cavity that can be accessed via two identical openings each presenting $n$ carbonyl groups. These molecules can act as a host for a wide variety of molecules due to a combination of the hydrophobic character of the CB cavity with the ionic-dipolar interactions that can be established between the guests and the carbonyl groups at the portals. Hence, $C B[n]$ can interact with metal ions, ${ }^{2}$ organic guests both neutral and charged, ${ }^{3}$ and in the case of $\mathrm{CB}[8]$, even form ternary complexes, hosting two guests simultaneously. ${ }^{4}$ These rich binding properties have been successfully exploited in drug delivery, ${ }^{5}$ switchable systems, ${ }^{6}$ and to create supramolecular polymers ${ }^{7}$ and networks. ${ }^{8}$ In addition, the portals can interact with substrates, in particular with gold and palladium surfaces. ${ }^{9}$ This can be used to induce $\mathrm{pH}$ and/or ion-responsive aggregation of nanoparticles, ${ }^{10}$ as well as enabling the fabrication of self-assembled monolayers (SAMs) of unmodified CB[n] on gold, ${ }^{11}$ both with potential applications for biosensing. Monolayers of $\mathrm{CB}$ [7] on gold surfaces have been used to reversibly immobilize biomolecules. ${ }^{12}$ However, these layers do not cover completely the gold surface, as was concluded from electrochemistry experiments. ${ }^{11}$ The exposed areas can potentially interact with other molecules in solution, introducing a non-specific contribution to the binding that may affect the performance of the SAMs.

Force spectroscopy (FS) has been used to study weak interactions quantitatively. ${ }^{13}$ The rupture force for the interaction between $\mathrm{CB}[6]$ and spermine has been reported to be $120 \mathrm{pN}$, although the kinetic behavior of this complex was not explored. ${ }^{14}$ Very recently computational studies have been performed in which the forced dissociation of several guests with $C B[n]$ molecules was modeled. ${ }^{15}$ However, experimental studies at the single molecule level over a dynamic range have not been reported. The study of interactions at the single molecule level allows to discriminate between different binding modes, in contrast with the results of ensemble techniques, which are frequently an average of all contributions. In this chapter the ability of self-assembled monolayers of $\mathrm{CB}$ [7] on gold to recognize a neutral hydrophobic guest, adamantane (Ad, see Scheme 4.1), is investigated using dynamic force spectroscopy (DFS) in order to discriminate between specific and unspecific interactions. 

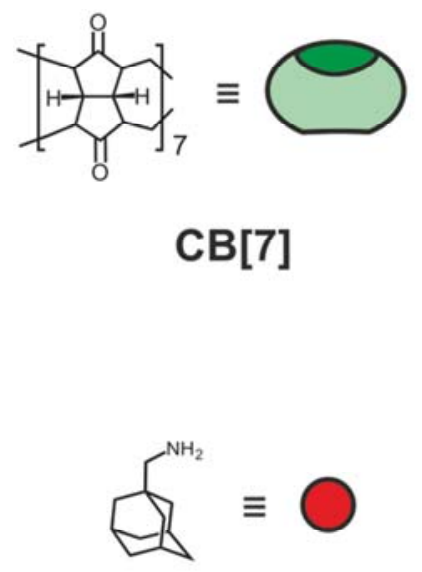

Ad

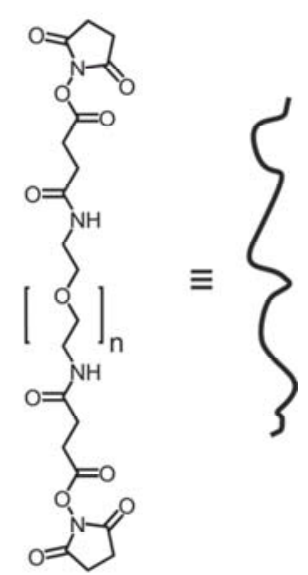

PEG 3000

Scheme 4.1 Compounds used in this chapter.

\subsection{Results and discussion}

A DFS experiment consists of repeatedly rupturing the studied complex in order to obtain statistical information about the strength of the interaction. ${ }^{13 b}$ To do so, one of the moieties (in this case the guest) is attached to the surface of an AFM cantilever, while the complementary moiety is attached to a supporting substrate. The experiments are carried out in liquid media to avoid the appearance of capillary forces between these two surfaces. The distance between the surfaces is controlled using a piezoelectric crystal. Starting from a distance of several hundreds of $\mathrm{nm}$, the distance is reduced until the tip contacts the substrate and exerts a certain amount of force on it (approach, see Figure 4.1). The force can be calculated by measuring the deflection of the AFM cantilever with a laser beam and calibrating its elastic properties. ${ }^{16}$ Once a preset positive force is achieved the system is maintained in this situation for a predetermined amount of time in order to allow the formation of complexes (dwell time). After that the distance is increased at a constant speed (retraction). During this retraction phase the cantilever initially relaxes towards its equilibrium position, however, if an interaction exists between the tip surface and the substrate a deflection in the opposite direction will be observed. When the elastic energy stored in the cantilever is big enough to 
break this interaction a sudden relaxation will be observed and the cantilever will travel undeflected until the initial point, from where the whole process can be repeated. Each of these approach-retract cycles is performed in a few seconds, and is usually plotted as a force-distance curve. However, if the moieties are attached directly to the surface the measured interaction will not originate exclusively from the studied complexes, but also from other contributions such as Van der Waals, electrostatic or hydrophobic forces between the tip and the substrate. For this reason a polymer chain is frequently used to connect the moieties to the surface. ${ }^{17}$ The advantages of this strategy are many. Firstly, any short-ranged unspecific interaction will be broken before the studied complex. Secondly, the characteristic non-linear elasticity of the polymer helps to discriminate ruptures originating from a single complex from others originating from several complexes in parallel. Finally, in some cases the formation of a complex requires a certain degree of freedom, which cannot be achieved using direct attachment to the surface. On the other hand, the loading rate (how fast the force is applied over the bond) is heavily influenced by the non-linear elasticity of the polymer, instead of being determined from just the retraction speed and the cantilever spring constant. ${ }^{18}$ This quantity is important since the rupture force depends on the loading rate. ${ }^{19}$ For this reason the slope of the force-curve in the points just prior to the rupture is used to calculate the instantaneous loading rate exerted on the complex at the moment of rupture.

The approach-retract cycles are acquired at different retraction speeds to examine the dependence of the rupture force with the loading rate. Polymer models such as the worm-like chain (WLC) or the freely-jointed chain (FJC) can be fitted to the non-linear region of each force-distance curve to discriminate the cases where only a single chain was being pulled. In some of the curves sawtooth patterns are observed when several complexes tethered by chains of different length are ruptured in series. In these cases, only the last rupture is analyzed to ensure that the observed instantaneous loading rate is only being exerted over one complex. Following the previously discussed strategy, a commercially available PEG crosslinker (PEG 3000, see Scheme 4.1) was used to connect an adamantanemethylamine guest (Ad) to amine functionalized AFM cantilevers. The host molecules (CB[7]) were immobilized on a clean gold substrate. ${ }^{11}$ In short, pre-cleaned gold substrates were immersed in a CB[7] aqueous solution ( $1 \mathrm{mM}$ ) for $24 \mathrm{~h}$ at room temperature. Since the dissociation of these complexes is a thermally activated process, microscopic fluctuations 
on the system affect the measurements. ${ }^{20}$ Thus, several hundreds of forcedistance curves need to be collected at each retraction speed to obtain statistically reliable values of the most probable rupture force and instantaneous loading rate. These most probable values were obtained from kernel density estimations constructed using the resulting data from these experiments. A well resolved two-peak force distribution was observed instead of the expected single peak (see Figure 4.2). Lateral tails of the distribution towards higher forces have been reported in other studies and attributed to diverse causes such as heterogeneity of chemical bonds ${ }^{21}$ or simultaneous ruptures of more than one complex. ${ }^{22}$ However, in the case of the $\mathrm{Ad}-\mathrm{CB}[7]$ complex the two peaks were more resolved than in those studies.

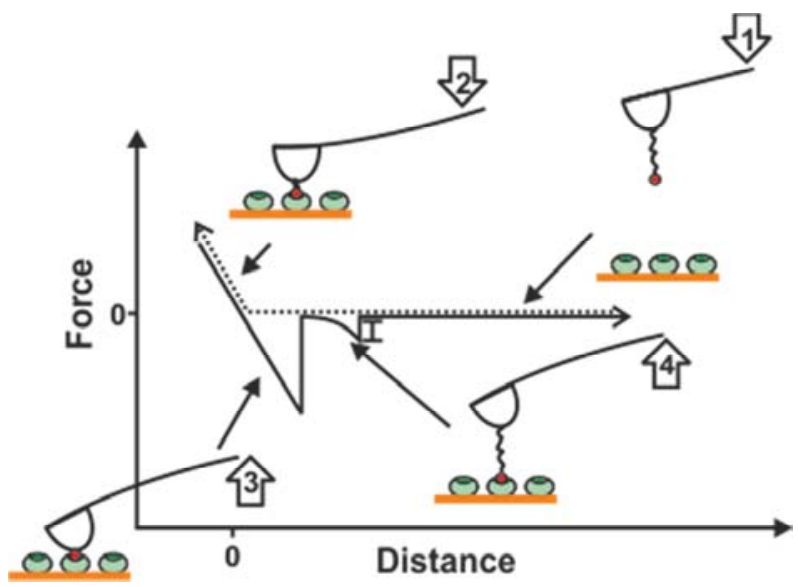

Figure 4.1 Force-distance curve using a tethered moiety. During the approach phase the cantilever is relaxed (1) until the tip contacts the substrate and is deflected (2). During the retraction a negative force (unspecific interaction) is detected with a constant slope while the tip and substrate are in contact (3). After this interaction is broken, a nonlinear region appears as the polymer chain is stretched (4). 

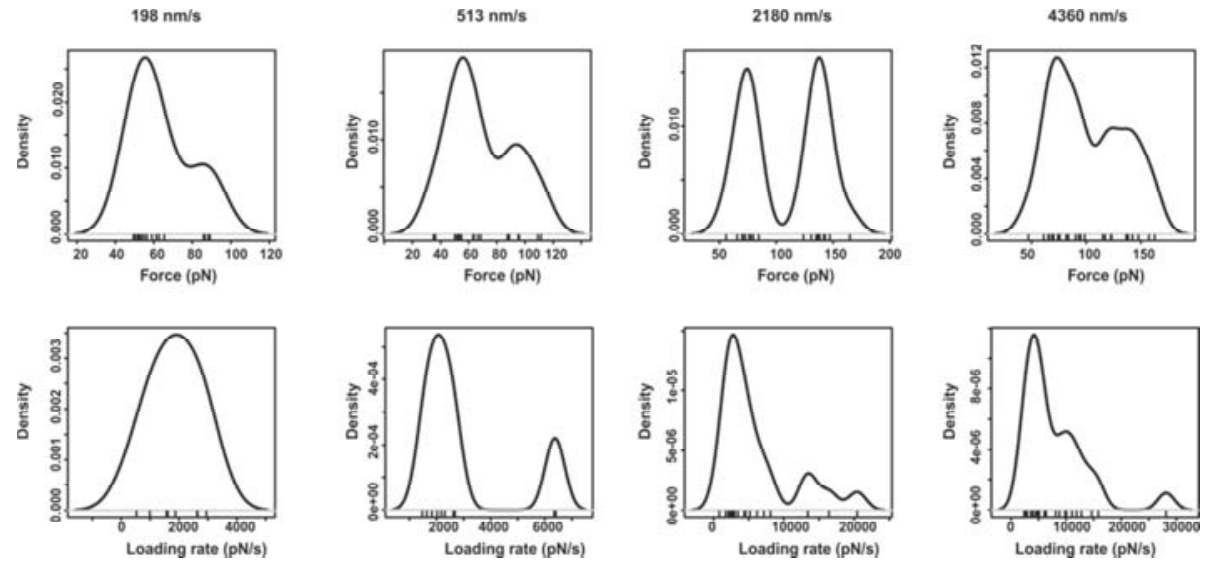

Figure 4.2 DFS results of the Ad-CB[7] complex. Density of probability for rupture force (top row) and instantaneous loading rate (bottom row) at different retraction speeds (columns). The ticks in the horizontal axis indicate individual measurements.
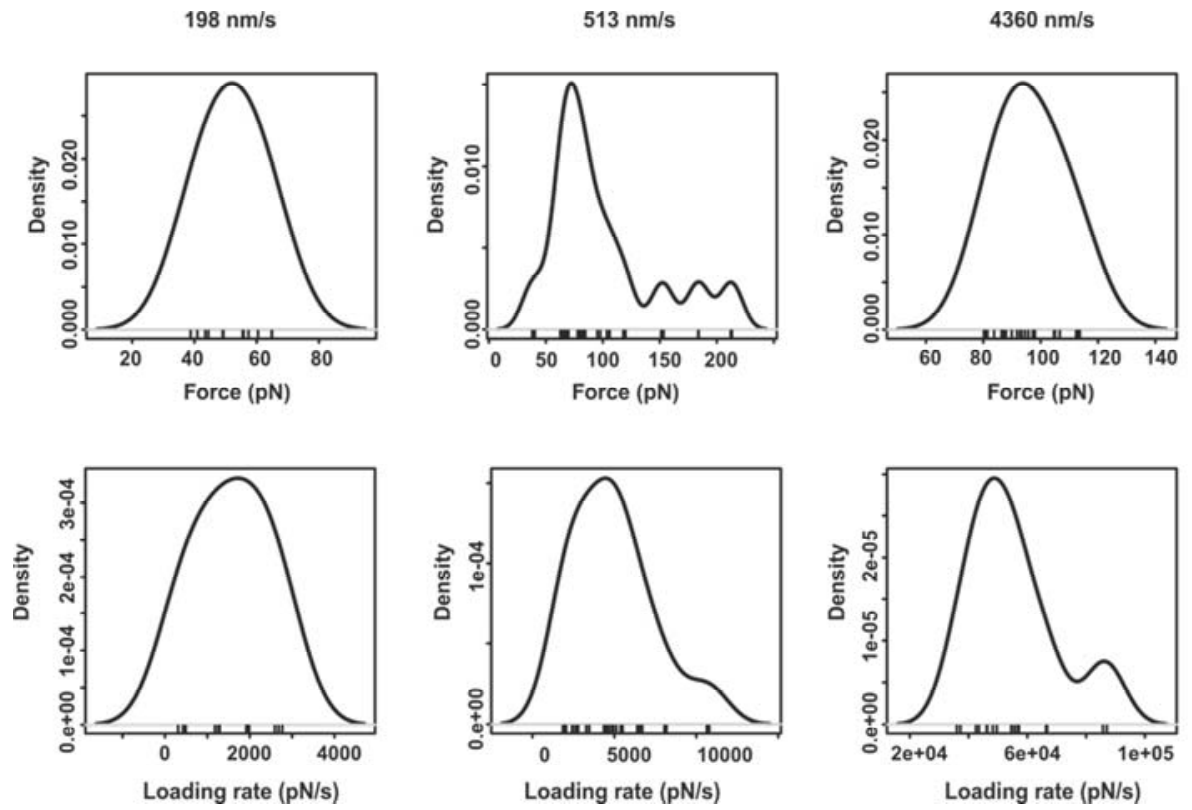

Figure 4.3 DFS results of the Ad-gold interaction. Density of probability for rupture force (top row) and instantaneous loading rate (bottom row) at different retraction speeds (columns). The ticks in the horizontal axis indicate individual measurements. 
Additional experiments were required in order to determine the origin of these two forces. Gold substrates were treated following the same procedure as for the formation of the $\mathrm{CB}$ [7] monolayer, but in this case the host molecules were not added to the solution. DFS experiments using Ad-functionalized tips and these gold substrates were performed to study the interaction of Ad with the gold surface exclusively. In this case only a single peak was observed in the kernel density plots of rupture force (see Figure 4.3).

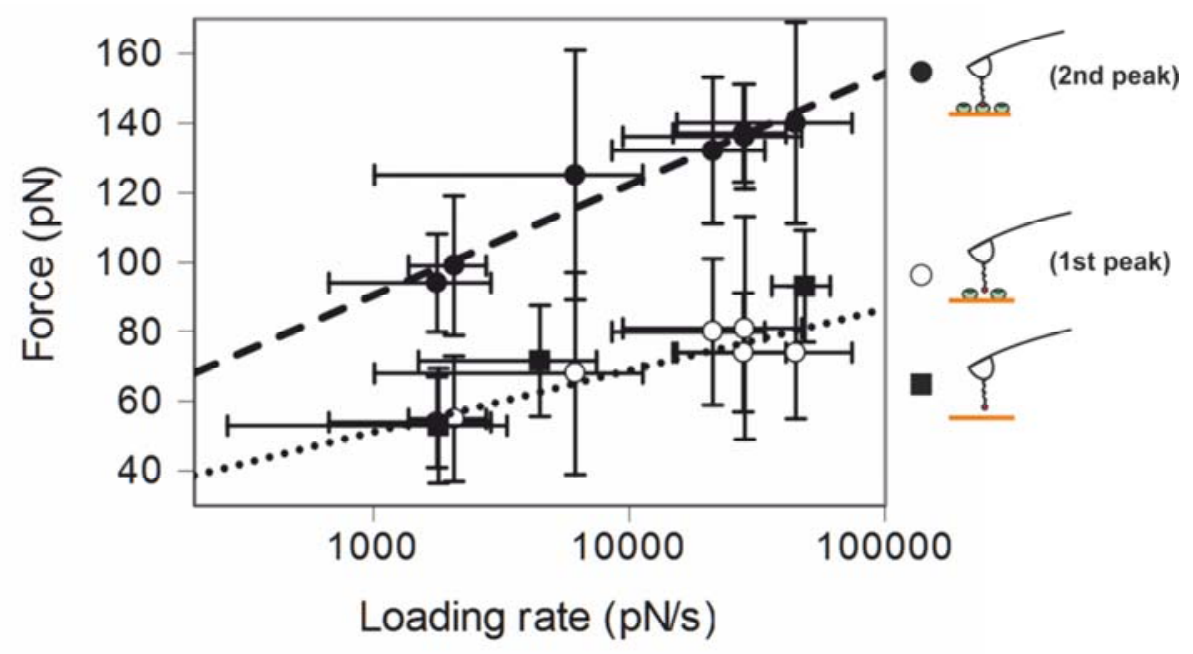

Figure 4.4 Results from DFS experiments using Ad-CB[7] and Ad-Au. The low (open circles) and high (filled circles) force peaks are plotted against the logarithm of the instantaneous loading rate. The data from Ad-Au (filled squares) is consistent with the low force peak observed in AdCB[7]. The lines are fits to Evans model.

A semilogarithmic plot of the most probable rupture forces versus the instantaneous loading rate showed a consistent trend for the two most probable rupture forces observed in Ad-CB[7] experiments (see Figure 4.4). When plotting the most probable rupture forces for the Ad-Au ruptures against the loading rates, the data points coincide with those points from the low force peak observed in the Ad-CB[7] experiments. Thus, the high force values can be attributed to ruptures of the host-guest interaction between $\mathrm{Ad}$ and $\mathrm{CB}$ [7]. This suggests that monolayers of $\mathrm{CB}$ [7] on gold are not well-packed and the empty $\mathrm{Au}$ substrate is accessible to the liquid medium. AFM images of $C B$ [7] monolayers were acquired to confirm this aspect (see Figure 4.5). The images 
show a patchy surface, where the elevated areas can be interpreted as regions covered with host molecules and the depressed areas as the bare gold substrate. The surface coverage of $\mathrm{CB}$ [7] has been previously estimated to be $48 \%$ using electrochemistry by others, ${ }^{11}$ suggesting an incomplete coverage of the gold substrate. This is in agreement with the interpretation of the DFS data and the AFM images of $\mathrm{CB}$ [7] monolayers on gold presented here.

The plot in Figure 4.4 shows a clear dependence of the rupture force with the loading rate for the interactions of $A d$ with gold and $C B$ [7]. This is characteristic of interactions probed out-of-equilibrium. A model describing this situation was developed by Evans and Ritchie. ${ }^{19}$ The most probable rupture force, $f^{*}$, increases with the loading rate, $\rho$.

$f^{*}(\rho)=\frac{k_{B} T}{x_{\beta}} \ln \left(\frac{\rho x_{\beta}}{k_{B} T k_{o f f}^{*}}\right)$

In this expression $k_{B} T$ is the thermal energy, $x_{b}$ is the width of the potential barrier of the interaction and $k_{\text {off }}^{*}$ is the dissociation rate constant at zero force (the intrinsic off-rate constant of the interaction). The lines in Figure 4.4 are fits of the model to the DFS data. In the case of the Ad-Au interaction a potential width $x_{B-A d-A u}$ of $0.48 \pm 0.08 \mathrm{~nm}$ and an off-rate $k_{\text {off-Ad-Au }}^{*} 0.3 \mathrm{~s}^{-1}$ (confidence interval $0.1-1.1 \mathrm{~s}^{-1}$ ) were estimated, whereas a potential width $x_{b-A d-C B[7]}$ of 0.34 $\pm 0.04 \mathrm{~nm}$ and off-rate constant $k^{*}{ }_{\text {off-Ad-CB[7] }}$ of $0.03 \mathrm{~s}^{-1}$ (confidence interval 0.008 $0.1 \mathrm{~s}^{-1}$ ) was found for the Ad-CB[7] interaction. Thus, the dissociation of the host-guest interaction is approx. 10 times slower that the dissociation of the guest-surface interaction. This suggests that the $\mathrm{Ad}-\mathrm{CB}[7]$ complex is more stable than the Ad-Au interaction, which presumably explains why other experiments using this type of monolayers did not observe the latter interaction. $^{11-12}$ Moreover, the guest in this study is not cationic, while the ferrocenium guests used in the cited studies are positively charged, making the differences in stability between the specific and unspecific interactions even larger since $\mathrm{CB}[\mathrm{n}]$ has been shown to bind preferably positively charged guests. ${ }^{3}$ The dwell time (100 ms) compared with the characteristic lifetimes of the Ad$\mathrm{Au}(3 \mathrm{~s}$ ) and $\mathrm{Ad}-\mathrm{CB}[7]$ (30 s) complexes suggests that once the Ad moiety binds either specifically or unspecifically it remains in that state until the interaction is mechanically broken. Assuming that the chance of association is similar in both situations, the average of the ratios between the number of specific Ad$\mathrm{CB}[7]$ ruptures and the total number of ruptures $(40 \pm 9 \%)$ and compares favorably to the reported $\mathrm{CB}$ [7] monolayer coverage of $48 \%{ }^{11}$ 

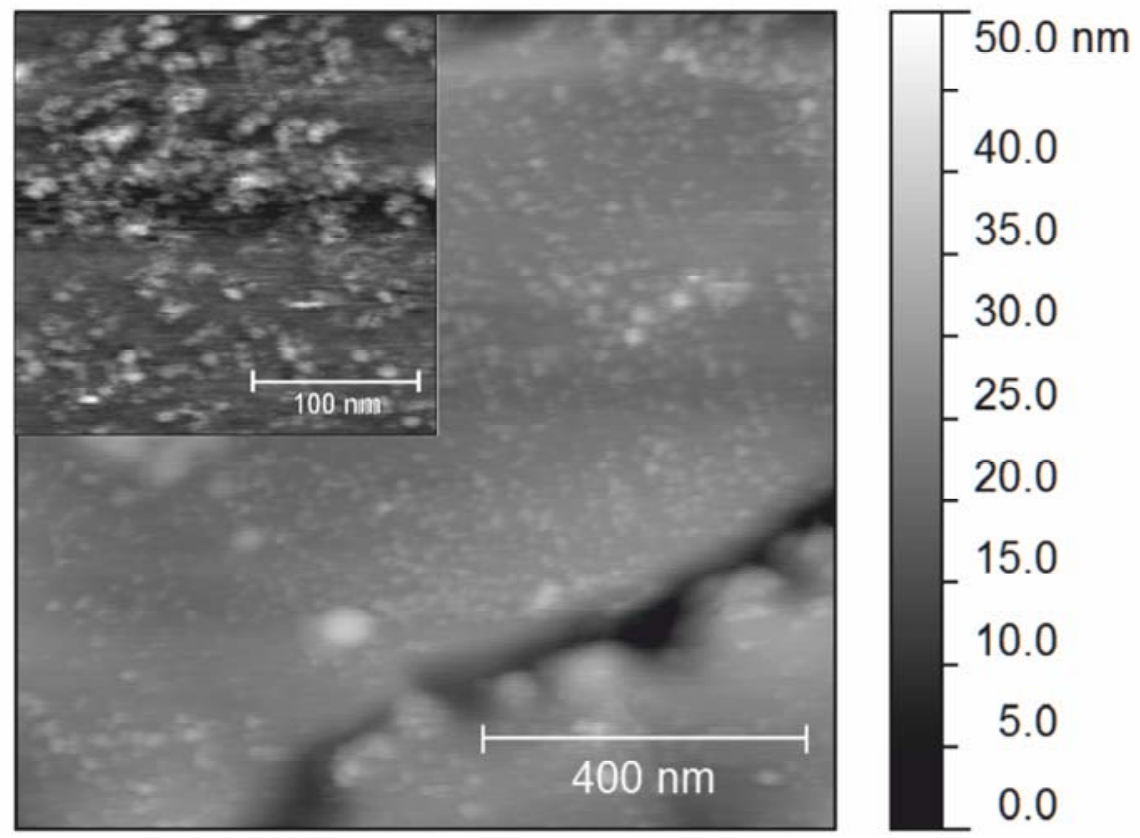

Figure 4.5 Tapping mode AFM images of $\mathrm{CB}[7]$ monolayers on gold acquired in liquid. $\mathrm{CB}$ [7] covered areas can be seen as white patches over the gold surface. The height scale for the inset is $0-10 \mathrm{~nm}$.

Finally, the equilibrium constant of the Ad-CB[7] binding could be calculated if the association rate constant were known. Unfortunately, few studies of the kinetics of $\mathrm{CB}[\mathrm{n}]$ systems have been reported, ${ }^{23}$ and none of them addressed the binding of $A d$ to $C B$ [7]. We can compare this interaction with the binding of Ad to $\beta$-cyclodextrin (Ad- $\beta C D)$. The driving force of the interaction is hydrophobicity in both cases and the radius of the cavity is slightly smaller in the case of $\mathrm{CB}$ [7] (6.0 $\AA$ for $\beta C D$ and $5.4 \AA$ for $C B[7]$ ), thus an upper limit for the association rate constant for $\mathrm{Ad}-\mathrm{CB}$ [7] should be the association rate constant of $A d-\beta C D$ complexes, which is on the order of $10^{8} \mathrm{M}^{-1} \mathrm{~s}^{-1} .^{24}$ Therefore, the maximum possible value for the equilibrium constant of $\mathrm{Ad}-\mathrm{CB}$ [7] should be $3 \cdot 10^{8} \mathrm{M}^{-1}$. Compare this value to the equilibrium constant for the complexation in solution of $\mathrm{CB}$ [7] and adamantane-1-carboxylic acid $\left(3.2 \cdot 10^{8} \mathrm{M}^{-1}\right){ }^{3}$ or to the one of $\mathrm{CB}$ [7] and 1-adamantanol $\left(2.8 \cdot 10^{10} \mathrm{M}^{-1}\right),{ }^{25}$ which should be both very similar interactions where no further stabilization of the complex due to charge interactions is expected. On the other hand, the complexation of $\mathrm{CB}[7]$ and 
amantadine, which is stabilized by the interaction between the protonated amine and the rim of $\mathrm{CB}[7]$, has an equilibrium constant of $4 \cdot 10^{12} \mathrm{M}^{-1}$. ${ }^{23 a}$ The equilibrium constants of complexes of $\mathrm{CB}$ [7] with other $\mathrm{NH}_{3}{ }^{+}$-functionalized adamantane guests have been reported to be higher than $10^{14} \mathrm{M}^{-1}$, ${ }^{25}$ well over the maximum value for the complex studied here. Additional studies of the association rate constants of this type of complexes are still needed for more meaningful comparisons between kinetic and thermodynamic constants of $\mathrm{CB}[7]$ systems.

\subsection{Conclusions}

The rupture force of $\mathrm{Ad}-\mathrm{CB}[7]$ complexes when the $\mathrm{CB}$ [7] molecules were attached to a gold substrate was measured. An additional characteristic force was identified as the rupture of the interaction between $\mathrm{Ad}$ and the gold interface. The dissociation rate constants of both interactions were calculated using a theoretical model. The comparatively high stability of the Ad-CB[7] interaction explains the successful use of this type of monolayers in other studies, where the unspecific interaction of the guests with the gold surface is too weak to affect the results.

\subsection{Experimental}

\section{Materials}

All solvents were purchased from commercial sources and used as received unless explicitly noted. Aminopropyldimethylethoxy silane (APDES), succinimidyl-[N-methyl]- (NHS) terminated PEG 3000 (NHS-PEG-NHS, MW 3000), cucurbit[7]uril and adamantanemethylamine and were purchased from $A B C R$ and Sigma-Aldrich.

\section{$C B[7]$ monolayers}

Gold substrates (Arrandee) were flame annealed under a high purity hydrogen flame, quenched in ultrapure water and introduced in an aqueous solution of $\mathrm{CB}$ [7] $(0.1 \mathrm{mM})$ for $24 \mathrm{~h}$ and room temperature. The substrates were rinsed with water before use. 


\section{AFM cantilever functionalization}

The AFM cantilevers (MLCT, Bruker) were repeatedly washed in dichloromethane (DCM) and methanol. The cantilevers were oxidized in oxygen plasma for $20 \mathrm{~s}$ and then immersed into a (1:200) solution of APDES in distilled dry toluene for $1 \mathrm{~min}$. Then, they were rinsed with copious amounts of DCM. The APDES terminated cantilevers were incubated for $2 \mathrm{~h}$ in a 200:1 DCM/ N,Ndiisopropylethylamine (DIPEA) solution with PEG 3000 linker (NHS-PEG-NHS, 5 $\mathrm{mg} / \mathrm{ml}, 1 \mathrm{mM}$ ) and rinsed with DCM. Finally, they were incubated for $2 \mathrm{~h}$ in a 1 $\mathrm{mM}$ solution of adamantane-methylamine in DCM and rinsed with the same solvent. The cantilevers were kept in ultrapure water until they were used.

\section{AFM force spectroscopy measurements}

All force measurements were performed with a commercial Multimode Picoforce SPM (Veeco, Digital Instruments, USA) using a liquid cell (Veeco) and milliQ water. The spring constants of the cantilevers were calibrated using the built-in thermal tune software. Force-distance curves were acquired by approaching and retracting the tip at speeds ranging $200-5000 \mathrm{~nm} / \mathrm{s}$ and a dwell time of $0.1 \mathrm{~s}$. The tip was laterally displaced over the substrate between each approach-retraction cycle, covering $1 \mu^{2}$, and the maximum force applied to the surface was kept under $500 \mathrm{pN}$. We could obtain up to 5000 curves per cantilever, limited by the appearance of high adhesion between the tip and surface and a large decrease in the chance of observing polymer stretching. This we attribute to deterioration of the monolayers due to the repeated contact or adsorption of contamination on the tip.

\section{Data analysis}

We selected relevant force curves using our own plug-in script developed for Hooke. ${ }^{26}$ Each curve was examined for sudden changes in force (rupture events), then the data prior to the last rupture (the unbinding event happening farthest away from the surface) were fitted using a modified freely-jointed chain (FJC) model with fixed Kuhn length of $7 \AA^{27}$ and the contour length was the fit parameter, and a worm-like chain (WLC) model where both the persistence length and the contour length were fit parameters. Rupture events were rejected or kept for further analysis based on the quality of the fit, which was assessed visually by comparing both fits, and numerically by comparing the fitted persistence length to the expected value of 3.5-3.8 $\AA$, in the case of WLC 
fits. In the case of the FJC fits, the averaged force difference from each data point to the corresponding fitted force was compared with the standard deviation of the measured force in the non-contact area. If the ratio of these two parameters is close to one, the difference between the fit and data can be explained by thermal noise. Pairs of rupture force and instantaneous loading rate were obtained from the valid events and an estimation of the rupture force and loading rate probability densities was obtained with the help of the statistical package $\mathrm{R}^{28}$ by using kernel density estimation with Epanechnikov kernels and a fixed bandwidth of $10 \mathrm{pN}$ in the case of force or an automatically selected bandwidth (Sheather and Jones algorithm) ${ }^{29}$ in the case of loading rate and contour length. The resulting densities are shown in Figure 4.2 and Figure 4.3.

\section{AFM imaging}

Topographic images were acquired using an AFM Nanoscope III, (Bruker) in tapping mode equipped with a $\mathrm{Si}_{3} \mathrm{~N}_{4}$ sharp tip (MSCT, Bruker) using an Escanner. The measurements were performed in milliQ water.

\subsection{References and notes}

1. (a) Lagona, J.; Mukhopadhyay, P.; Chakrabarti, S.; Isaacs, L., Angew. Chem. Int. Ed. 2005, 44, 4844-70; (b) Lee, J. W.; Samal, S.; Selvapalam, N.; Kim, H.-J.; Kim, K., Acc. Chem. Res. 2003, 36, 621-30.

2. (a) Jeon, Y.-M.; Kim, J.; Whang, D.; Kim, K., J. Am. Chem. Soc. 1996, 118, 9790-1; (b) Whang, D.; Heo, J.; Park, J. H.; Kim, K., Angew. Chem. Int. Ed. 1998, 37, 78-80.

3. Liu, S.; Ruspic, C.; Mukhopadhyay, P.; Chakrabarti, S.; Zavalij, P. Y.; Isaacs, L., J. Am. Chem. Soc. 2005, 127, 15959-67.

4. (a) Kim, H.-J.; Heo, J.; Jeon, W. S.; Lee, E.; Kim, J.; Sakamoto, S.; Yamaguchi, K.; Kim, K., Angew. Chem. Int. Ed. 2001, 40, 1526-9; (b) Sun, S.; Gao, W.; Liu, F.; Li, F.; Fan, J.; Peng, X., Phys. Chem. Chem. Phys. 2011, 13, 570-5.

5. Walker, S.; Kaur, R.; Mclnnes, F. J.; Wheate, N. J., Mol. Pharmaceutics 2010, 7, 2166-72.

6. Choudhury, S. D.; Mohanty, J.; Pal, H.; Bhasikuttan, A. C., J. Am. Chem. Soc. 2010, 132, 1395-401. 
7. (a) Liu, Y.; Shi, J.; Chen, Y.; Ke, C.-F., Angew. Chem. Int. Ed. 2008, 47, 7293-6; (b) Liu, Y.; Yu, Y.; Gao, J.; Wang, Z.; Zhang, X., Angew. Chem. Int. Ed. 2010, 49, 6576-9.

8. Appel, E. A.; Biedermann, F.; Rauwald, U.; Jones, S. T.; Zayed, J. M.; Scherman, O. A., J. Am. Chem. Soc. 2010, 132, 14251-60.

9. (a) Lee, T.-C.; Scherman, O. A., Chem. Commun. 2010, 46, 2438-40; (b) Cao, M.; Lin, J.; Yang, H.; Cao, R., Chem. Commun. 2010, 46, 5088-90.

10. (a) de la Rica, R.; Velders, A. H., Small 2011, 7, 66-9; (b) Feng, D.; Zhang, Y.; Shi, W.; Li, X.; Ma, H., Chem. Commun. 2010, 46, 9203-5.

11. An, Q.; Li, G.; Tao, C.; Li, Y.; Wu, Y.; Zhang, W., Chem. Commun. 2008, 1989-91.

12. Young, J. F.; Nguyen, H. D.; Yang, L.; Huskens, J.; Jonkheijm, P.; Brunsveld , L., ChemBioChem 2010, 11, 180-3.

13. (a) Neuman, K. C.; Nagy, A., Nat. Methods 2008, 5, 491-505; (b) Bizzarri, A. R.; Cannistraro, S., Chem. Soc. Rev. 2010, 39, 734-49.

14. Kim, J.; Kim, Y.; Baek, K.; Ko, Y. H.; Kim, D.; Kim, K., Tetrahedron 2008, 64, 8389-93.

15. (a) Yu, J.-S.; Wu, F.-G.; Tao, L.-F.; Luo, J.-J.; Yu, Z.-W., Phys. Chem. Chem. Phys. 2011, 13, 3638-41; (b) Gilson, M. K., J. Chem. Theory Comput. 2010, 6, 637-46.

16. (a) Hutter, J. L.; Bechhoefer, J., Rev. Sci. Instrum. 1993, 64, 1868-73; (b) Sader, J. E.; Chon, J. W. M.; Mulvaney, P., Rev. Sci. Instrum. 1999, 70, 3967-9.

17. Hinterdorfer, P.; Kienberger, F.; Raab, A.; Gruber, H. J.; Baumgartner, W.; Kada, G.; Riener, C.; Wielert-Badt, S.; Borken, C.; Schindler, H., Single Mol. 2000, 1, 99-103.

18. Ray, C.; Brown, J. R.; Akhremitchev, B. B., J. Phys. Chem. B 2007, 111, 1963-74.

19. Evans, E.; Ritchie, K., Biophys. J. 1997, 72, 1541-55.

20. Bell, G. I., Science 1978, 200, 618-27.

21. Raible, M.; Evstigneev, M.; Bartels, F. W.; Eckel, R.; Nguyen-Duong, M.; Merkel, R.; Ros, R.; Anselmetti, D.; Reimann, P., Biophys. J. 2006, 90, 3851-64.

22. Gu, C.; Kirkpatrick, A.; Ray, C.; Guo, S.; Akhremitchev, B. B., J. Phys. Chem. C 2008, 112, 5085-92.

23. (a) Isaacs, L., Chem. Commun. 2009, 619-29; (b) Mock, W. L.; Shih, N. Y., J. Am. Chem. Soc. 1989, 111, 2697-9.

24. Novo, M.; Granadero, D.; Bordello, J.; Al-Soufi, W., J. Incl. Phenom. Macrocycl. Chem. 2010, 11, 173-88.

25. Moghaddam, S.; Yang, C.; Rekharsky, M.; Ko, Y. H.; Kim, K.; Inoue, Y.; Gilson, M. K., J. Am. Chem. Soc. 2011, 133, 3570-81. 
26. Sandal, M.; Benedetti, F.; Brucale, M.; Gomez-Casado, A.; Samori, B., Bioinformatics 2009, 25, 1428-30.

27. Oesterhelt, F.; Rief, M.; Gaub, H. E., New J. Phys. 1999, 1, 6.1-11.

28. RDC-Team, http://www.R-project.org 2008.

29. Sheather, S. J.; Jones, M. C., J. Roy. Stat. Soc. B Met. 1991, 683-90. 


\section{Chapter 5}

\section{Probing multivalent}

interactions in a synthetic host-
guest complex by dynamic
force spectroscopy

In this chapter the stability and strength of multivalent bonds is addressed using force spectroscopy techniques employing a synthetic adamantane/b-cyclodextrin model system. Comparing the experimental findings to theoretical predictions for the rupture force and the kinetic off-rate, when the valency of the complex is increased from monoto di- or trivalent, a transition occurred from quasi-equilibrium, with a constant rupture force of $99 \mathrm{pN}$, to a kinetically dependent state, with loading-rate-dependent rupture forces from 140 to $184 \mathrm{pN}$ (divalent) and 175 to $210 \mathrm{pN}$ (trivalent). Additional binding geometries, parallel monovalent ruptures, single-bound divalent ruptures and single and double-bound trivalent ruptures, have been identified. The experimental kinetic offrates of the multivalent complexes show that the stability of the complexes is greatly increased with the number of bonds, in agreement with the predictions of a multivalent model without allosteric cooperativity contributions. 


\subsection{Introduction}

Multivalency describes the interaction between multivalent receptors and ligands. It plays a pivotal role in biochemistry, governing many interactions between proteins and small molecules, between proteins or antibodies and cell membranes, between viruses and cells, etc. ${ }^{1}$ At interfaces in particular, multivalency is poorly understood in a quantitative sense. Understanding of multivalent interactions at interfaces thus constitutes a way to better understand and control biological recognition events ${ }^{2}$ and provides a tool to develop supramolecular nanomaterials. ${ }^{3}$

In principle, the usage of single-molecule techniques allows more detailed insight in the study of molecular interactions, in comparison with the traditional ensemble methods, such as calorimetry or NMR, on the macroscopic scale. Methods based on atomic force microscopy (AFM) and optical tweezers are more suited to register individual binding events and are employed to observe covalent and non-covalent bonds under controlled stress. ${ }^{4}$ In particular, dynamic force spectroscopy (DFS) enables the determination of kinetic dissociation rate constants by analyzing the studied bond rupture at different loading rates, i.e. how fast the bond is loaded with the external force. ${ }^{5}$ Lifetimes of molecular complexes and even a detailed description of the energy landscape for some biological systems have been deduced using this technique. ${ }^{6}$ Besides the study of complexes, the structure and unfolding pathways of proteins and mutant analogues such as $\operatorname{titin}^{7}$ or the folding of the Parkinson-involved protein $\alpha$-synuclein ${ }^{8}$ have been also examined using force spectroscopy. Moreover, modifications of this technique allow the construction of recognition maps on cellular membranes ${ }^{9}$ or study the effect of mechanical stress on chemical reactions. ${ }^{10}$

To our knowledge, only three studies of multivalency at the single molecule level by means of DFS have been reported before. ${ }^{11}$ The complexes used for these studies were biological (concanavalin A-mannose, ${ }^{11 a}$ MUC1 antigenantibody $\left.^{11 b}\right)$ and synthetic $\left(C_{60}\right.$-porphyrin tweezers $\left.{ }^{11 c}\right)$. Both studies using biomolecules concluded that the force of a multivalent bond scaled sublinearly with the number of bonds, whereas the $\mathrm{C}_{60}$-porphyrin system yielded a divalent rupture force stronger than two times the monovalent rupture force. Presumably, this discrepancy could originate from the fact that the binding of $\mathrm{C}_{60}$ to porphyrin tweezers is likely to be not only multivalent but also strongly 
allosteric, ${ }^{12}$ which would make the complex stronger than an independent binding site multivalent attachment. On the other hand, the experiments using MUC1 antigen-antibody studied ruptures of parallel bonds, which have been predicted to break at reduced forces if the linkers connecting them are of different length. ${ }^{13}$ In the case of the concanavalin A-mannose system conformational changes in the protein upon binding could change the affinity for successive bindings. In this study we use a synthetic host-guest system aiming to avoid the potential problems described above. The binding sites are physically different molecules, which ensures that the affinities for the second and successive bonds are unaffected by the first, and the geometry of the multivalent guests is such that only one linker is pulled.

In our laboratory, we use the adamantane/ $\beta$-cyclodextrin $(\beta C D)$ supramolecular complex as a model system to study multivalency. ${ }^{14} \beta C D$ is an oligosaccharide consisting of seven glucose units connected in a ring shape. The inner cavity of this ring is hydrophobic and can accommodate a wide variety of guest molecules. ${ }^{15}$ This host-guest couple has been used by us for fabricating various nanostructures, and detailed SPR work in combination with thermodynamic models has provided insight in the multivalent characteristics of this interaction. ${ }^{16}$ Previously, employing DFS we reported the rupture force between a series of monovalent guests bound to an AFM tip and surface-bound $\beta C D$. The notion of thermodynamic equilibrium allowed correlating quantitatively the measured pull-off force values with free binding energy of the complexes. ${ }^{17}$

Here we provide a thermodynamic and kinetic analysis of the binding of mono-, di- and trivalent guests to self-assembled monolayers of $\beta C D$ host. The interactions between single pairs of $\beta C D$ and adamantane as well as between divalent and trivalent assemblies of these molecules were studied using DFS to study their binding forces and kinetic off-rates. A theoretical model for the multivalent assembly is used to compare the measured multivalent off-rates with the expected monovalent off-rate.

\subsection{Results and discussion}

\subsubsection{Monovalent guest}

In a typical force spectroscopy (FS) experiment several force curves are acquired using a functionalized AFM tip and/or substrate. Thus, $\beta C D$ and 
adamantyl-functionalized molecules (Scheme 5.1) need to be immobilized in a convenient manner, one over the surface of the AFM tip and the other on a supporting substrate.

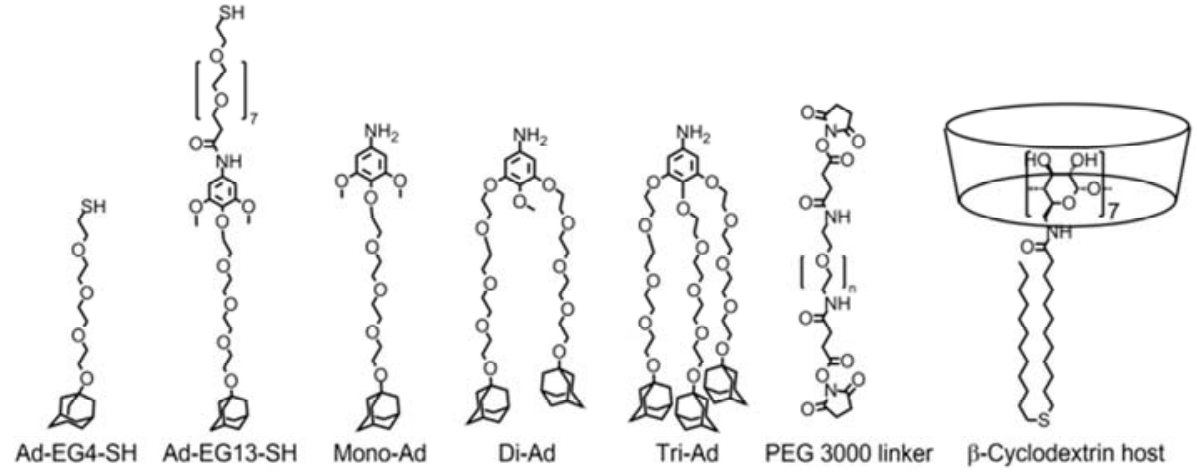

Scheme 5.1 Compounds used in this chapter.

We followed a well-known procedure to create a densely packed monolayer of $\beta C D$ over a gold substrate, also known as the molecular printboard. ${ }^{18}$ These printboards expose the hydrophobic cavities of $\beta C D$ in a hexagonal lattice with a lattice constant of ca. $2 \mathrm{~nm}$ to the medium, effectively acting as a surfacebound multivalent receptor. The adamantyl (Ad) guests are readily attached to gold-coated AFM tips using thiol groups, either in a single-step procedure, when using short linkers, or successively connecting amine-reactive linkers and amino-functionalized guest moieties to a cysteamine monolayer (Scheme 5.2 and see the experimental section for a detailed description of the procedures for functionalizing the tip and substrate).

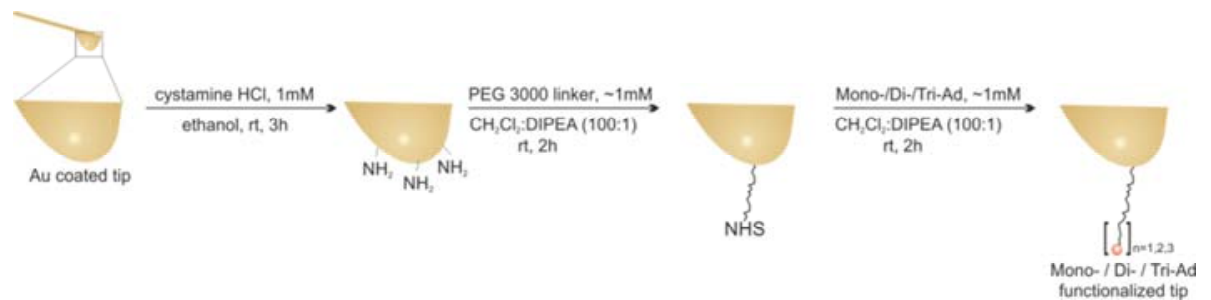

Scheme 5.2 Tip functionalization with Mono-Ad Di-Ad or Tri-Ad using PEG3000 linker. 
For the study of multivalent guests, the spacers between the two Ad moieties must be chosen to be long enough to allow for multivalent complexation on the molecular printboard. ${ }^{19}$ The functionalized tip and supporting substrate are installed in the AFM setup for the DFS experiment. A laser is employed to monitor the deflection of a soft cantilever connected to the tip. The distance between the base of this cantilever and the substrate is controlled by means of a piezoelectric crystal. Starting from a situation where the tip and substrate are not in contact, the distance between them is reduced (approach) until a positive deflection is detected. After a chosen delay during which tip and surface remain in contact, the separation between substrate and cantilever base is progressively increased (retract). During this step a negative deflection can be observed, which is related to an interaction between the tip and surface. When the energy accumulated in the bent cantilever is enough to break this interaction, the measured maximum deflection can be multiplied by the cantilever spring constant to give the unbinding force of the studied interaction. On a typical curve like the ones shown in Figure 5.1A the retraction starts at the far left, and interactions with the surface are being broken until achieving the flat zero-force region, representing a relaxed cantilever.

Typically in FS studies the moieties of interest are not directly connected to the AFM tip surface, instead a flexible linker is introduced between them to allow some translational and rotational freedom for complexation. In addition, this linker, if long enough, allows the rupture of the specific interaction pair to happen when the tip is several $\mathrm{nm}$ away from the surface and any non-specific interactions between tip and surface have been already overcome. Furthermore, the characteristic non-linear elasticity of the linker can be used as an internal check of the measurements. A force curve obtained using such linkers shows multiple ruptures. The first rupture event, a sudden change in the measured force, usually originates from unspecific short-range interactions between the tip and substrate. Subsequent events originate from the studied moieties being ruptured and should show a non-linear region, characteristic of the particular linker in use, prior to the rupture. The slope of this curve immediately before the rupture is the instantaneous loading rate that is being applied to the bond (Figure 5.1B). Thus, the choice of a linker between an AFM tip and the guest molecules largely influenced the availability of rate data in our previous DFS studies, ${ }^{17}$ where short linkers (alkyl chains of 7-18 carbons) were used. Moreover, achieving and proving that the ruptures originate from a single host-guest pair is much more difficult with such sort linkers. Therefore the use 
of longer tethers between the monovalent guest and the tip surface is explored here. We chose three different water soluble ethyleneglycol (EG) based linkers, i.e. a short tetraethyleneglycol (Scheme 5.1A, EG4) linker, an EG13 linker (Scheme 5.1A) of intermediate length and a long linker containing a polyethyleneglycol (PEG) chain of $M_{w} 3000 \mathrm{~g} / \mathrm{mol}$ (Scheme 5.1A, PEG 3000, approx. 68 ethyleneglycol units). Force-distance curves obtained in aqueous media $^{20}$ were examined selecting only those events that represent a polymer stretch region matching the expected single PEG chain behavior, which was used as an internal check of the measurements. A curve for each of these linkers is shown in Figure 5.1A, where it can be clearly seen how the separation between the first and final ruptures increases with the linker length. In addition, only in the case of the longest linker non-linear stretching behavior was unequivocally observed and could be reliably fitted to a worm-like chain (WLC) model ${ }^{21}$ to obtain instantaneous loading rates, whereas in the case of the shorter linkers the transition in stiffness occurs too fast yielding too few data points for a reliable fitting procedure. With these results in hand, the PEG $\mathbf{3 0 0 0}$ linker was employed in the remainder of this chapter.

The collected force-distance curves, obtained from experiments where the monovalent adamantyl guest was linked to the tip via the PEG 3000 linker, were fitted to a WLC polymer model, where the contour length was the fitting parameter and the persistence length was fixed at a value of $3.5 \AA$, corresponding to half of the Kuhn length of $7 \AA^{22}$ The fitted contour length (typically between $10-50 \mathrm{~nm}$ ) was reasonably close to the expected value for a PEG chain of $3000 \mathrm{~g} / \mathrm{mol}$, (average $25 \mathrm{~nm}$ ), considering the polydispersity of the linker and the fact that we selected the last rupture event, which relates to the longest available linker on the tip. Then, the instantaneous loading rate was calculated from the slope of the WLC fit at the rupture point (Figure 5.1B) to give pairs of force - loading rate values. For these forces and loading rates an estimated density of probability was constructed. Such density graphs (Figure $5.2 \mathrm{~B}, \mathrm{C})$ show what forces and loading rates were observed for each set of pulling conditions. On some of the force probability densities a long high-force tail or even a well-defined secondary peak was found (Figure 5.2C). Crossing these data with the loading rate data, where also a long tail was present, we found that data points can be grouped into two quadrants (Figure 5.2A). One group consists of low force/low rate data points, which relate to monovalent force, and another group consists of high force/high rate data points, which we tentatively attribute to the parallel rupture of two monovalent host-guest pairs. 
In this latter case the rupture force value has been predicted to vary with the difference in length of the chains that link the two guests, being lower when the two pairs are linked by chains of different lengths as compared to the situation when both chains are of identical length. ${ }^{13}$
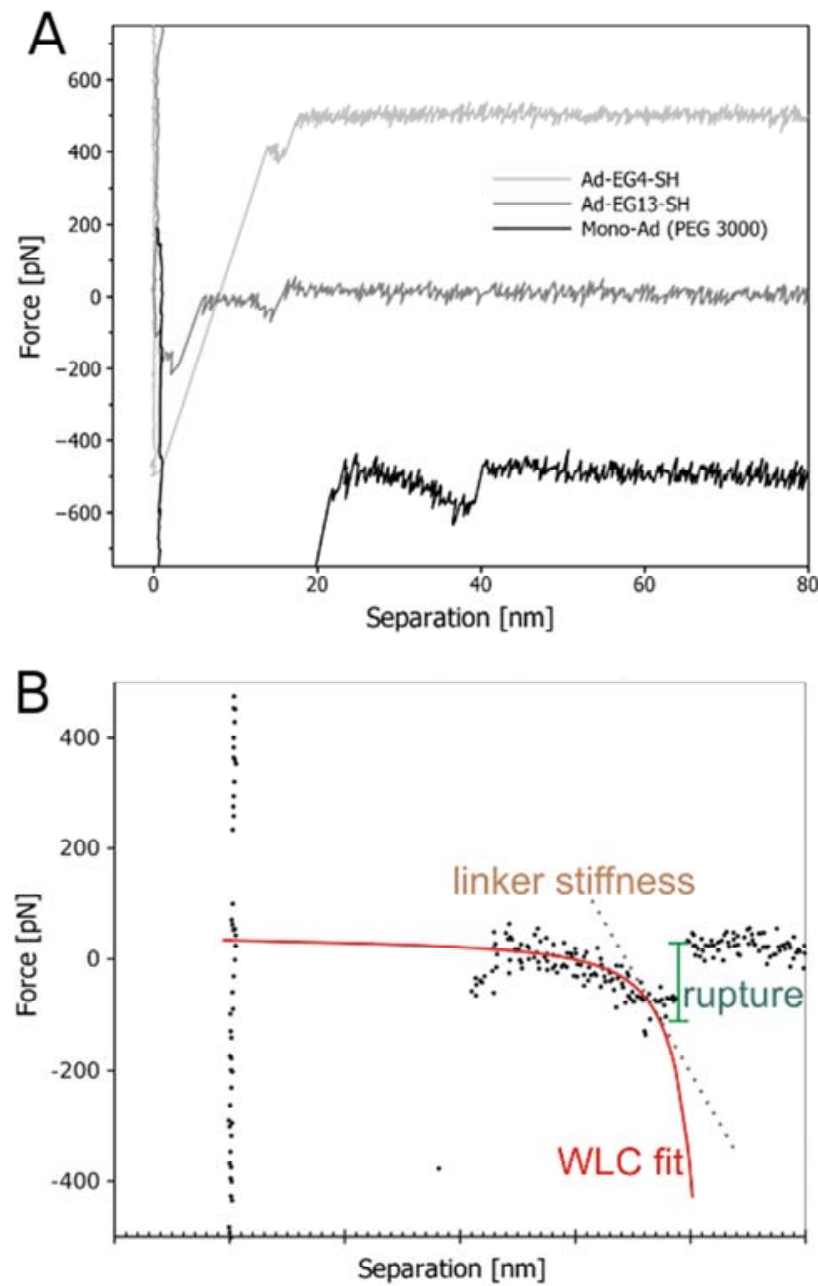

Figure 5.1 A) Characteristic force-separation curves (offset in force axis for clarity) for monovalent adamantyl guests linked to the AFM tip by a short tetra ethylene glycol linker (top), linker with 13 EG units (middle) or a long PEG linker of 3000 MW, EG 68 (bottom). B) Example of a curve with highlighted rupture force and WLC fit to the data. The slope of this fit at the rupture point quantifies the stiffness of the polymer, and together with the retraction speed allows the calculation of the instantaneous loading rate. 
To perform further analysis on the monovalent data we made use of a theory developed by Evans et al. ${ }^{5}$ based on previous work by Bell, ${ }^{23}$ which predicts linear scaling of the rupture force $f$ to the logarithm of the loading rate $r$, where $k_{B}$ is the Boltzmann constant, $T$ the temperature, $\Delta x$ the width of the energy barrier and $k_{\text {off }}$ the intrinsic dissociation rate (the dissociation rate constant when the bond is not loaded by an external force).

$$
f=\frac{k_{B} T}{\Delta x} \ln \left(\frac{r \Delta x}{k_{o f f} k_{B} T}\right)
$$

When the data of the monovalent guest were plotted in this way (open symbols in Figure 5.2D), a zero slope was observed in the explored loading rate range, indicating that the complex can be considered in thermodynamic equilibrium for our experimental conditions, in agreement with our previous studies on monovalent guests ${ }^{17,24}$ and other systems ${ }^{25}$. In addition, the most apparent rupture force in the case of the monovalent adamantane guest, $97 \pm 19 \mathrm{pN}$, closely matches our previous results obtained for short tethers $(102 \pm 15 \mathrm{pN}) \cdot{ }^{17}$ This loading rate independent behavior can be explained by the fact that the binding and unbinding rate constants for the cyclodextrin-adamantane system ( $k_{\text {on }}$ is diffusion limited, on the order of $10^{8} \mathrm{M}^{-1} \mathrm{~s}^{-1}$ for many $\beta C D$ guests ${ }^{26}$ and $k_{\text {off }}$ estimated to be $2 \cdot 10^{3} \mathrm{~s}^{-1}$ for a measured equilibrium constant ${ }^{16 a} K_{e q}=4.6$. $10^{4} \mathrm{M}^{-1}$ ) represent a very rapid equilibration compared to the timescale of the AFM measurements. The monovalent guest probed under these conditions can be considered at thermodynamic equilibrium and thus no kinetic effects such as the unbinding force dependence on loading rate are expected to show up in the measurements. This aspect could be confirmed by implementing a model ${ }^{27}$ which predicts the transition from equilibrium to a dynamic regime. The estimated potential of the adamantane-cyclodextrin interaction was introduced in such model (see Appendix A), obtaining an equilibrium rupture force of 90 $\mathrm{pN}$ and an unforced off-rate of $2 \cdot 10^{3} \mathrm{~s}^{-1}$, which are very close to the values presented above. The loading rate required to observe the characteristic logarithmic increase of rupture forces is estimated as $10^{7}-10^{8} \mathrm{pN} / \mathrm{s}$, well beyond range of AFM based DFS. Loading rate dependence could not be assessed for the parallel monovalent unbinding events (filled symbols in Figure 5.2D), since all ruptures occurred at similar loading rates. 
A

B
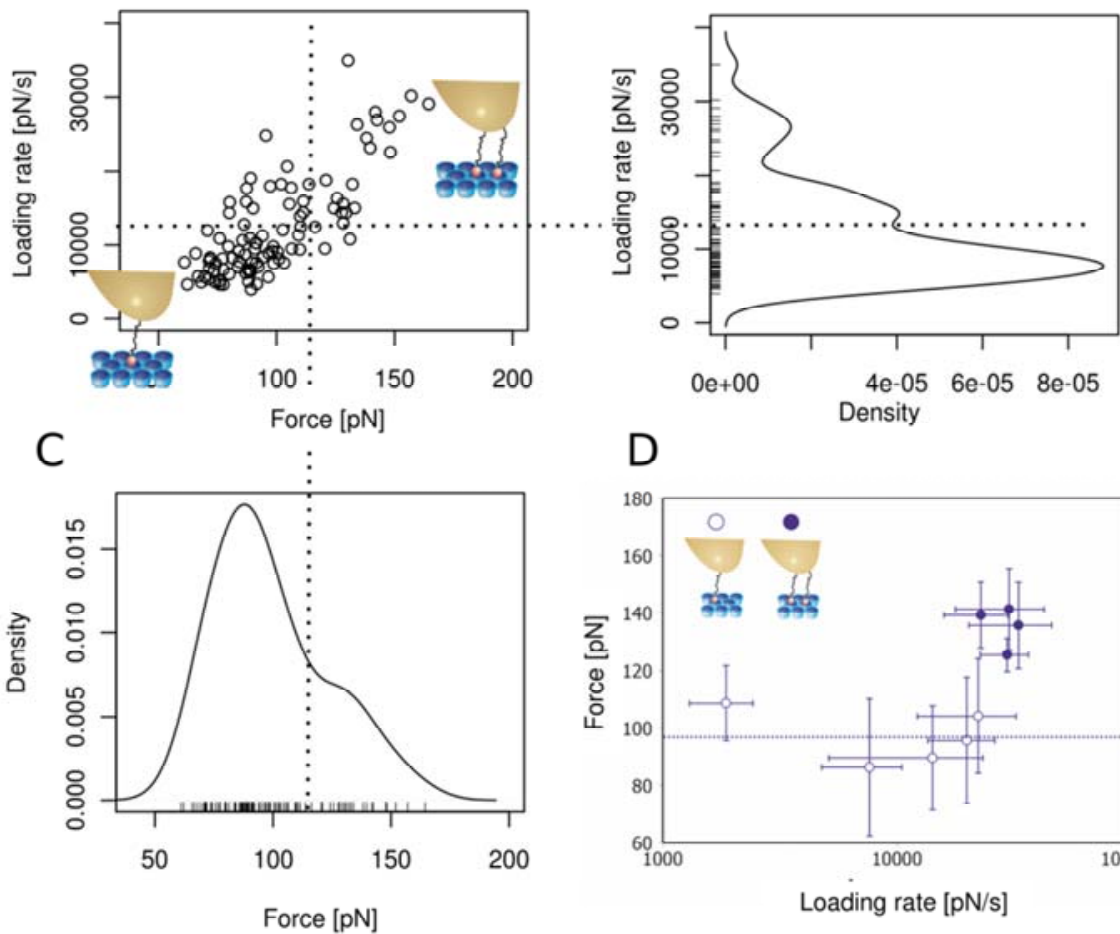

D

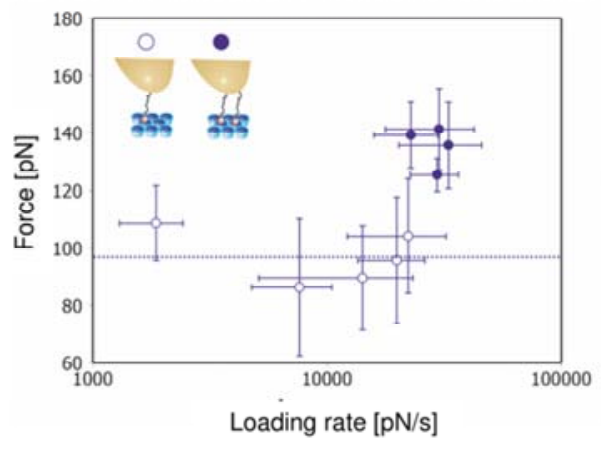

Figure 5.2 Analysis of monovalent guest DFS data obtained using PEG 3000 linker. A) Pairs of force and loading rate obtained from the collected curves and $B, C$ ) kernel smoothened densities of probability constructed from them (the ticks indicate individual occurrences). Data corresponding to the main peaks of force and loading rate are assigned to monovalent binding, and the data corresponding to the high-force, high-rate tails of the distributions to monovalent (parallel) binding. D) Combined results from different retracting speeds; the dotted line is the averaged rupture force of a monovalent complex. 
A
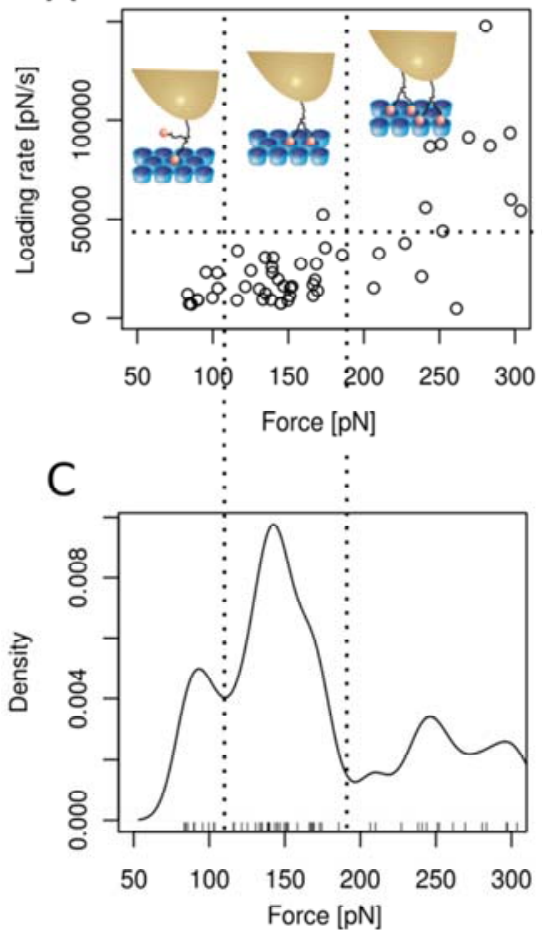

B

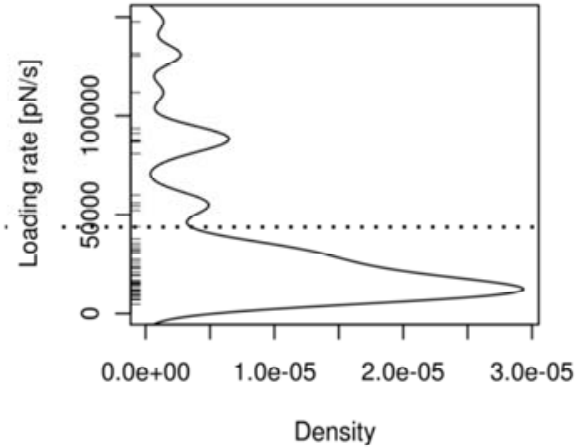

D

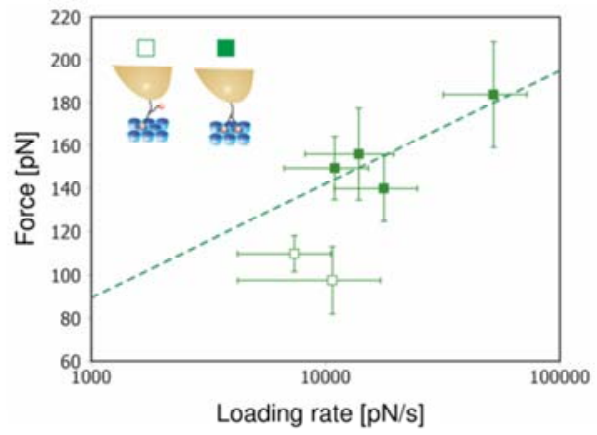

Figure 5.3 Analysis of divalent guest DFS data. A) Pairs of force and loading rate obtained from the collected curves. B,C) kernel smoothened densities of probability constructed from them (the ticks indicate individual occurrences). The main peak on the force distribution is assigned to divalent force (divalent-double, filled symbols), and the secondary peak at lower force (when present) to pulloffs were only a single adamantane was bound in the instant of rupture (divalent-single, open symbols). Data corresponding to the high-force, high-rate tails originates from several possible situations were more than one divalent host-guest complex was ruptured. Notice that since only one linker is involved in divalent-single and divalent-double ruptures, both fall in the same band of loading rates. D) Combined results from different retracting speeds; the dashed line is a logarithmic fit as a guide to the eye. 


\subsubsection{Divalent guest}

Following the same attachment procedure using the PEG 3000 linker to the AFM tip, force-distance curves for the divalent guest were collected and analyzed (Figure 5.3A). In strong contrast to the monovalent guest, the most probable rupture force was found to be dependent on the loading rate, ranging from $140 \pm 15 \mathrm{pN}$ (probe retraction speed $260 \mathrm{~nm} \mathrm{~s}^{-1}$ ) to $184 \pm 25 \mathrm{pN}$ (probe retraction speed $1160 \mathrm{~nm} \mathrm{~s}^{-1}$ ). The rupture forces for divalent guests were in all cases smaller than twice the rupture force for monovalent guests, in agreement with theoretical predictions ${ }^{28}$ and experimental studies. ${ }^{11 a, b, 13}$ However, when crossing the distributions of rupture force and loading rate (Figure 5.3) three regions can be clearly distinguished. The most probable loading rate comprised two characteristic rupture force peaks. The lower of these two peaks indicates a rupture force matching the values of the previously determined monovalent unbinding force, and can be interpreted as events where only one of the adamantyl moieties was bound at the moment of rupture (named as divalentsingle in the rest of this chapter). The higher and most probable force corresponds to divalent ruptures (divalent-double). Finally, a third region consists of high-force, high-loading rate events, which we attribute to parallel unbinding of two (or more) divalent guests. Since these data can originate from several binding situations (double+double, single+single and single+double bound for two parallel divalent guests) further analysis was not performed.

One important aspect revealed by these experiments is the difference between multivalent and parallel arrangements. It has been previously reported that the difference in length between two linkers in a parallel rupture will lead to lower rupture forces. ${ }^{13}$ In the case of probing two host-guest complexes the maximum rupture force will be measured when the linkers are loaded under identical conditions at the moment of the rupture. This implies that both chains are of the same length, which is not likely when using long polymeric linkers, and their attachments points are such that both distances to the surface are equal. The geometry of our divalent guest is the optimal approach to achieve simultaneous and equal loading, since the two host-guest pairs are connected by a single linker and branch out only at the very end. The measured higher rupture forces in the case of the divalent guest (approx. $170 \mathrm{pN}$ at $30 \mathrm{nN} / \mathrm{s}$ ) when compared to parallel monovalent guests (approx. $140 \mathrm{pN}$ at $30 \mathrm{nN} / \mathrm{s}$ ) confirms the prediction that linking the guest moieties through a single tether is the optimal approach to measure multivalent interactions. 
When more than one weak moiety is attached in a multivalent fashion the dissociation rate constant is expected to decrease several orders of magnitude, ${ }^{16 b}, 29$ opening the possibility to probe the complex in an out-ofequilibrium regime. This is confirmed here by measuring rupture forces for divalent-double cyclodextrin-adamantane complexes, which presented a defined loading rate dependency.

\subsubsection{Trivalent guest}

Experiments using the trivalent guest were performed in the same manner as described above. The density of probability of measured rupture forces showed multiple peaks over a large range of forces (see Experimental section). We discussed above that high force peaks originate from parallel ruptures. In the case of two trivalent guests rupturing simultaneously the forces can not only be relatively high (triple+triple, triple+double, double+double) but also appear in a range of forces very near divalent characteristic forces (single+single) and between divalent and trivalent forces (single+double). However, we have shown above that these parallel ruptures can be filtered out by selecting only the events corresponding to the main peak of the loading rate distribution (Figure 5.4).

After such a procedure the remaining events were plotted and three distinct force regions were identified (Figure 5.5). Comparing these forces and their instantaneous loading rates with the previous results from the mono- and divalent guests, (Figure 5.6), we assigned the lower, middle and higher force peaks to ruptures where one (trivalent-single), two (trivalent-double) or three (trivalent-triple) adamantyl moieties, respectively, were bound. The force for the ruptures of trivalent-single complexes were in good agreement with the measured unbinding force for a monovalent complex of ca. $100 \mathrm{pN}$, independent of the loading rate. Moreover, the ruptures of trivalent-double complex showed the same trend and values than the divalent-double data, which validates our interpretation of the force density graphs. The rupture force of a fully-bound trivalent complex depended on the loading rate, with values ranging from $175 \pm 18 \mathrm{pN}$ (probe retraction speed $581 \mathrm{~nm} \mathrm{~s}{ }^{-1}$ ) to $210 \pm$ $20 \mathrm{pN}$ (probe retraction speed $2620 \mathrm{~nm} \mathrm{~s}^{-1}$ ), in all cases stronger than the measured forces for the divalent complex at comparable loading rates. 

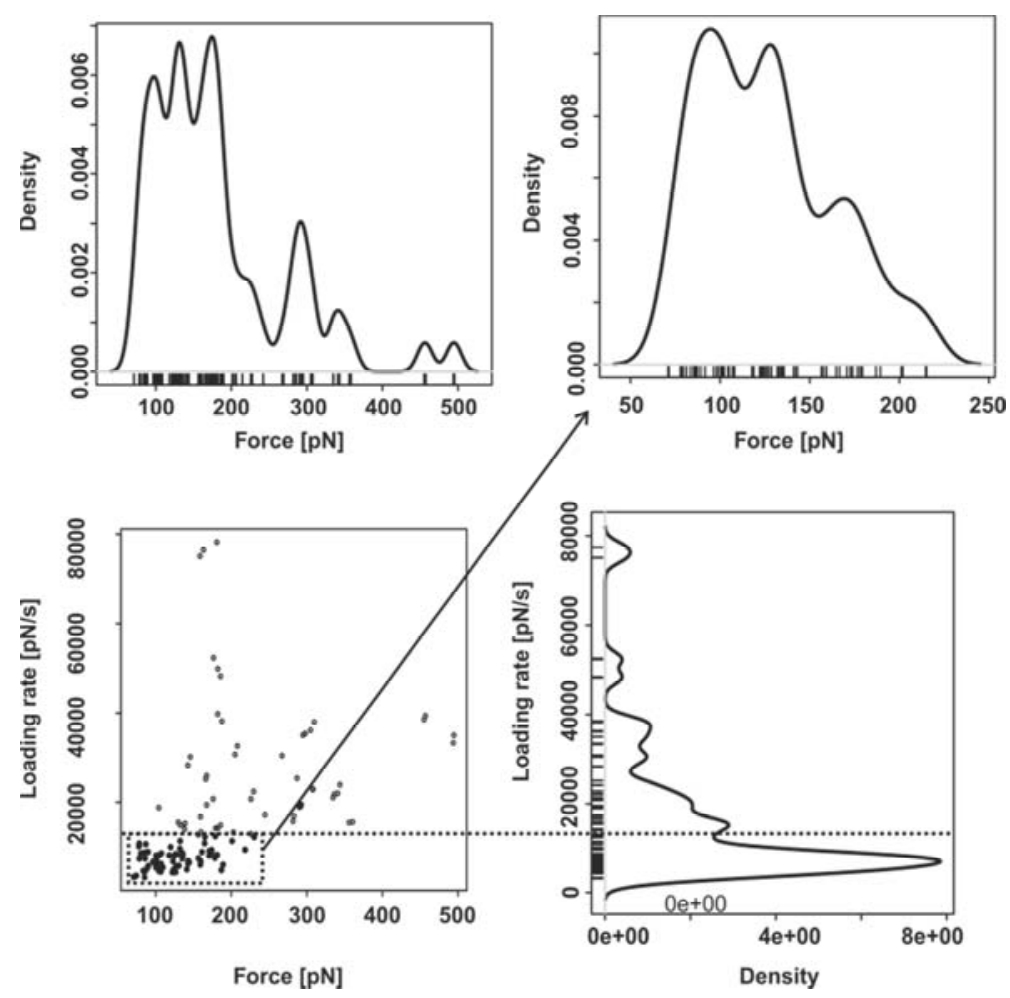

Figure 5.4 Example of the data analysis for the trivalent guest, PEG $\mathbf{3 0 0 0}$ linker. Only the forces corresponding to the main peak of loading rate are selected. A) Density of probability of rupture force, B) Density of probability of selected rupture forces, C) Force versus loading rate scatter plot and D) Density of probability of loading rate. 

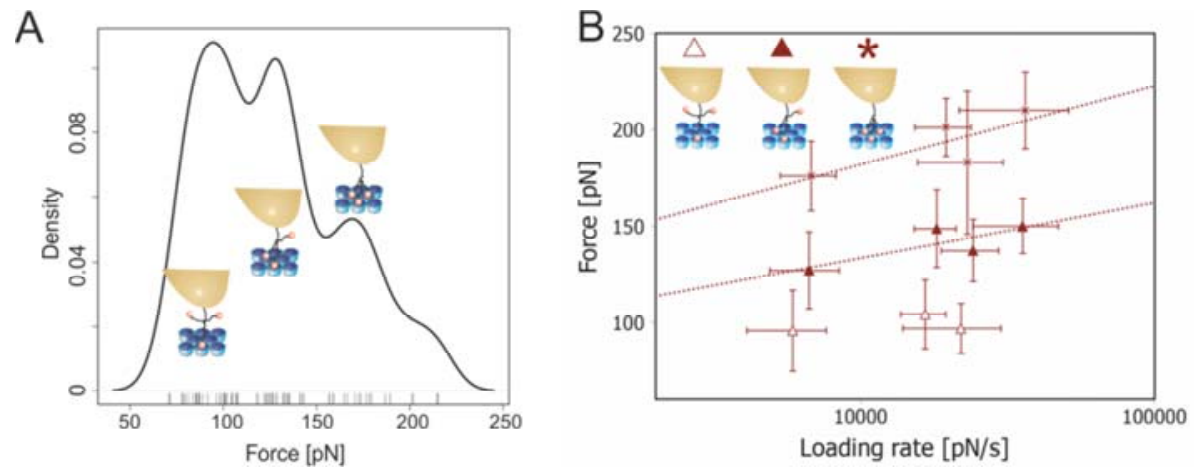

Figure 5.5 Results from DFS experiments using trivalent guest. A) Kernel smoothened density of probability of rupture forces corresponding to the main peak of the loading rate distribution (the ticks indicate individual occurrences). The three different peaks are assigned (from lower to higher force) to ruptures of trivalent- single (open symbols), double (filled symbols) and triple (stars) bound guest. B) Combined results from different retracting speeds; the dotted lines are logarithmic fits for the second and third peaks as a guide to the eye.

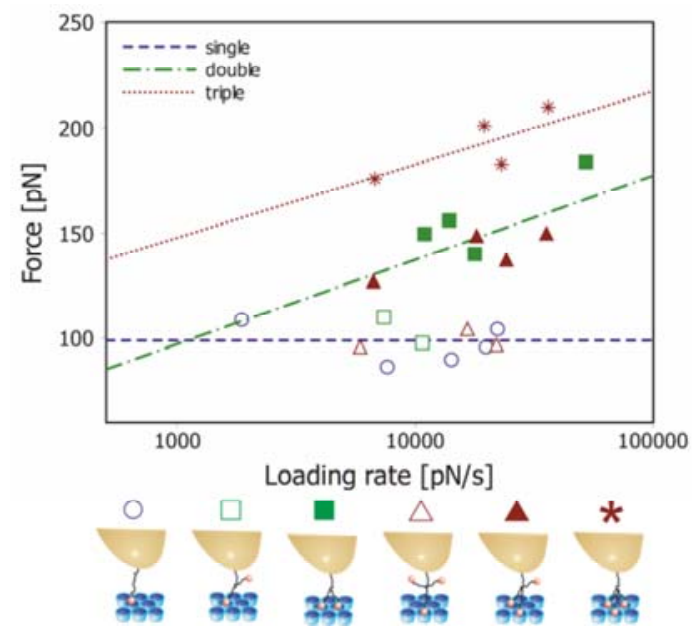

Figure 5.6 Comparison of AFM-DFS of mono- di- and tri- adamantyl guests. Different attachment geometries are assigned, single bound mono- di- and trivalent (open symbols), double bound di- and trivalent (filled symbols) and triple bound trivalent (stars). The lines are least squares fits to the Evans model for the double and triple bound data, and the average rupture force for the single bound data. 


\subsubsection{Kinetic parameters}

The monovalent adamantyl- $\beta C D$ system appears to be at equilibrium under the accessible conditions for loading rate and acquisition bandwidth. Therefore, the Bell-Evans model (Eq. 5.1), developed for dissociations occurring out-ofequilibrium, cannot be applied to our data in order to obtain a value for $k_{\text {off }}$ for the monovalent system based on force spectroscopy. However, the experimental data from divalent and trivalent guests shows a pronounced loading rate dependence of the rupture forces, making them suitable for a fit. The values of $\Delta x$ and $k_{\text {off }}$ for ruptures of double (from divalent-double and trivalent-double data) and triple (from trivalent-triple data) attachments are presented in Table 5.1. The increase in stability of the complex with increasing valency is clear, with lifetimes extended 100 - to 10000 -fold per each extra bond. Such enhanced stability is expected from multivalent complexes and it is one of the main reasons why multivalent strategies can be employed when designing molecules for medical applications. ${ }^{30}$

Table 5.1 Parameters obtained fitting FS data to Bell-Evans model. For $\boldsymbol{k}_{\text {off }}$ a confidence interval is given in parenthesis.

\begin{tabular}{|c|c|c|c|}
\hline Bond valency & $\begin{array}{c}\text { Monovalent } \\
\text { (estimated) }\end{array}$ & Double & Triple \\
\hline$\Delta \boldsymbol{x}(\mathrm{nm})$ & $0.2-0.3$ & $0.24 \pm 0.09$ & $0.27 \pm 0.10$ \\
\hline $\boldsymbol{k}_{\text {off }}\left(\mathbf{s}^{-1}\right)$ & $2000^{31}$ & 0.2 & 0.004 \\
& & $(0.01-3.5)$ & $(0.0003-0.05)$ \\
\hline
\end{tabular}

\subsubsection{Comparison of DFS results with theoretical model for multivalent dissociation}

A model predicting the thermodynamics and kinetics of multivalent assemblies has been proposed ${ }^{16 b}$ and validated for divalent host-guest systems like the one in our study. ${ }^{16 a}$ The multivalent binding/unbinding process can be described in a stepwise manner as depicted in Scheme 5.3, an intermolecular binding is followed by successive intramolecular steps where the intrinsic binding affinity 
is unchanged. This model explains the enhanced binding affinity by a high local effective concentration $\left(C_{\text {eff }}\right)$ of the host that is available for the divalent guest molecule when it is bound through only one of its guest moieties (divalentsingle). Thus no cooperativity in the sense of an increase of intrinsic binding affinity from the first to the second binding (allosteric cooperativity, see Chapter 2 of this thesis) is assumed. ${ }^{32}$ The expected equilibrium constant for a multivalent complex can be related to the corresponding monovalent constant according to this scheme. For clarity the trivalent rates and equilibrium constants are here given the subscript ' $T$ ' and their monovalent and divalent counterparts the subscript ' $M$ ' and ' $D$ ' respectively. In the case of a divalent complex we have:

$$
K_{e q-D}=\frac{2 k_{o n-M} \cdot k_{o n-M} C_{e f f}}{k_{o f f-M} \cdot 2 k_{o f f-M}}=K_{e q-M}^{2} C_{e f f}
$$

Since the association rate in this system is diffusion limited, we propose $\mathrm{k}_{\mathrm{on}}=$ $10^{8} \mathrm{M}^{-1} \mathrm{~s}^{-1}$ in the case of the monovalent adamantyl/ $\beta C D$ system. ${ }^{26}$ In the case of the divalent guest, a statistical factor 2 is introduced.

$$
\begin{gathered}
k_{o n-D}=2 k_{o n-M} \\
K_{e q-D}=\frac{k_{o n-D}}{k_{o f f-D}}
\end{gathered}
$$

We can combine Eqs. 5.2-5.4 to obtain a relationship between the monovalent and divalent off-rate constants

$$
\frac{k_{o f f-M}}{k_{o f f-D}}=\frac{1}{2} K_{e q-M} C_{e f f}
$$

The values for $K_{\text {eq-M }}\left(4.610^{4} \mathrm{M}^{-1}\right)$ and $C_{\text {eff }}(0.2 \mathrm{M})$ were determined in previous studies $^{16 a}$ using the same $\beta C D$ and adamantyl host-guest system. In particular, the value of $\mathrm{C}_{\text {eff }}$ indicates that the second guest moiety is only able to complex with the (approx. 6) host cavities directly near the already occupied cavity. Thus, we can estimate the value for the unforced off-rate of a monovalent guest; 


$$
k_{o f f-M(\text { estimated })}=\frac{k_{o n-M}}{K_{e q-M}}=2 \cdot 10^{3} s^{-1}
$$

Substituting this value in Eq. 5.5 for a model-based estimation of the unforced off-rate of a divalent guest gives:

$$
k_{\text {off }-D(\text { estimated })}=\frac{2 \cdot k_{\text {off }-M(\text { estimated })}}{K_{e q-M} C_{e f f}}=0.47 s^{-1}
$$

This estimated value for the divalent off-rate is remarkably close to the value $\left(0.2 \mathrm{~s}^{-1}\right)$ found after fitting the Evans model to our DFS data. The strong agreement between these two values for $k_{\text {off-D }}$ confirms that the enhanced unbinding force for a double-bound guest can be fully attributed to chelate cooperativity, which was our main aim when designing the structure of the guest molecules.

A similar derivation can be done for the trivalent guest, in this case we introduce in the last step (Scheme 5.3) the parameter $C^{\prime}{ }_{\text {eff }}=C_{\text {eff }} / 3$, since there are only two accessible cavities for the third guest moiety once a double bond has been established. Taking this into account we estimated the off-rate of a trivalent guest to be:

$$
k_{\text {off }-T(\text { estimated })}=\frac{9 \cdot k_{\text {off }-M(\text { estimated })}}{\left(K_{e q-M} C_{e f f}\right)^{2}}=2 \cdot 10^{-4} s^{-1}
$$

The difference between this value and the experimentally obtained $k_{\text {off- } T}\left(4 \cdot 10^{-3}\right.$ $\mathrm{s}^{-1}$ ) can presumably be explained taking in account the conformation of the ethyleneglycol linkers in a fully-bound trivalent complex (see Appendix B). The formation of the first cycle limits the available length of linker for the second intramolecular binding, which is an example of interannular cooperativity (see Chapter 2 of this thesis). Nevertheless, the introduction of an additional bond stabilizes the complex over a hundredth-fold compared to the fully bound divalent complex, as expected from the binding of a multivalent ligand on independent binding sites. 


$$
\begin{aligned}
& \stackrel{k_{o n-M}}{\stackrel{k_{o r r M}}{\rightleftarrows}} \\
& K_{\text {oq } M}=k_{o n-M} / k_{o t h M} \\
& K_{\text {op:D }}=k_{\text {on:D }} / k_{\text {oft. D }} \\
& K_{\text {eq-T }}=k_{\text {on } T} / k_{\text {olt } T}
\end{aligned}
$$
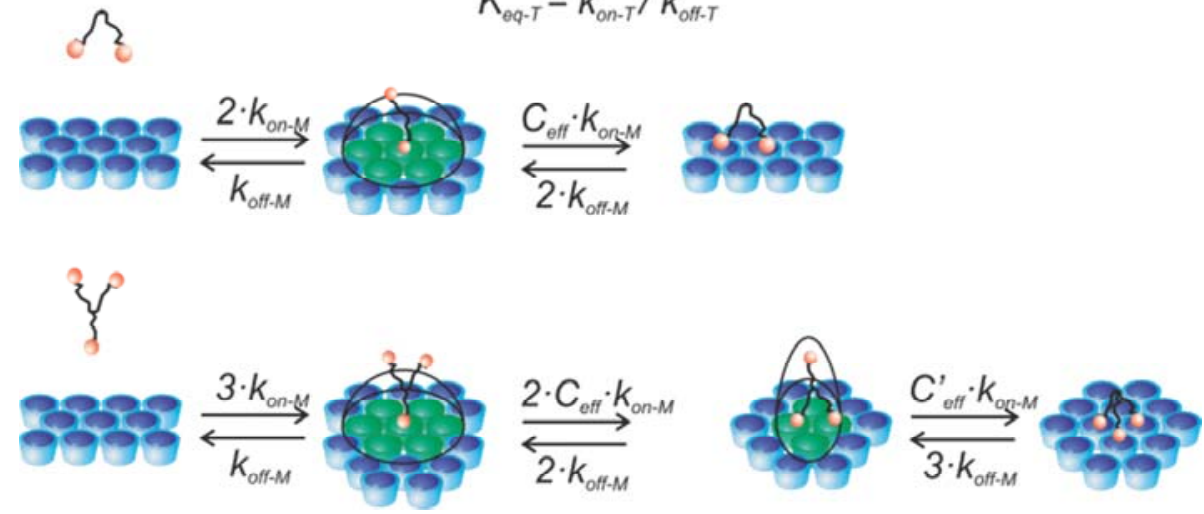

Scheme 5.3 Diagram of multivalent equilibrium and stepwise kinetics.

\subsection{Conclusions}

The rupture forces of mono- and di- and trivalent complexes of the $\beta$ cyclodextrin-adamantyl host-guest pair were measured. The monovalent complex was probed at equilibrium while the divalent and trivalent showed a significant loading rate dependency. The rupture forces of the fully bound divalent and trivalent complexes was found to be less than twice and thrice, respectively, the one of the monovalent complex. However, the transition between the equilibrium regime (monovalent) and the kinetic regime (divalent and trivalent) so far prevents a more meaningful comparison of these rupture forces to further confirm a particular scaling law.

Additionally, events where two parallel monovalent complexes were dissociating could be identified. Although the two single binding moieties are chemically identical to the case of a fully bound divalent complex, differences of length or load between the two parallel tethers lowered the measured rupture force with respect to the rupture force of a divalent complex. The geometry of the multivalent guests employed in this study, with only one tether pulling at the complex, allowed to achieve a situation equivalent to two or three equal parallel tethers, an optimal condition for obtaining more reliable results when studying multivalent interactions. Ruptures of lower force, 
matching the forces of monovalent or divalent ruptures, were observed in some of the experiments using multivalent guests. This suggests that at the moment of rupturing the multivalent guests were partially bound to the CD printboard.

Finally, the initial assumption for the studied system, non-allosteric multivalency, was confirmed by the kinetic off-rate values obtained from the analysis of the DFS experiments performed using multivalent adamantyl guests.

\subsection{Experimental}

\section{Materials}

All solvents were purchased from commercial sources and used as received. Cystamine hydrochloride, PEG 3000 (succinimidyl-[N-methyl]- (NHS)terminated homobifunctional PEG, $M_{W}$ 3000) and 2-mercaptoethanol were purchased from Sigma-Aldrich. $\beta C D$ adsorbate was synthesized as described previously $^{18}$. Water was purified and deionized (MQ water, resistivity of 18.2 $\left.\mathrm{M} \Omega \mathrm{cm}^{-1}\right)$.

All moisture-sensitive reactions were carried out under an argon atmosphere. ${ }^{1} \mathrm{H}$ NMR and ${ }^{13} \mathrm{C}$ NMR spectra were recorded on a Varian Unity INOVA (300 $\mathrm{MHz}$ ) spectrometer. ${ }^{1} \mathrm{H}$ NMR chemical shift values (300 MHz) are reported as $\delta$ using the residual solvent signal as an internal standard $\left(\mathrm{CDCl}_{3}, \delta 7.257\right) .{ }^{13} \mathrm{C}$ NMR chemical shift values $(75 \mathrm{MHz}$ ) are reported as $\delta$ using the residual solvent signal as an internal standard $\left(\mathrm{CDCl}_{3}, \delta\right.$ 77.0). Matrix-assisted laser desorption ionization (MALDI) TOF mass spectra were recorded using a Perkin Elmer/PerSpective Biosystems Voyager-DE-RP MALDI-TOF mass spectrometer. Analytical TLC was performed using Merck prepared plates (silica gel 60 F-254 on aluminum). Merck silica gel (40-63 $\mu \mathrm{m}$ ) was used for flash chromatography. Column chromatography was carried out on Merck silica gel 60 (230-400 mesh). Compound $\mathbf{4}$ was synthesized according to a literature procedure. ${ }^{19 a}$ The synthetic routes for the mono- and diadamantane functionalized anilines are according to Scheme 5.5 starting from the commercially available (Sigma) phenols (Scheme 5.4). 

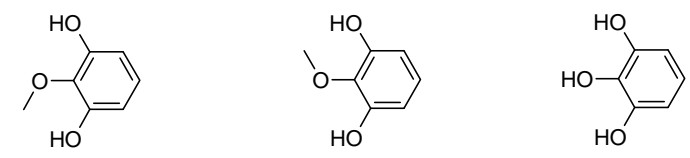

\section{Scheme 5.4}

1-[2-(2-\{2-[2-(Adamantan-1-yloxy)ethoxy]ethoxy\}ethoxy)-ethoxy]-2,6dimethoxybenzene (6). A suspension of 2,6-dimethoxyphenol 5 (0.226 g, 1.47 $\mathrm{mmol}), 4(0.510 \mathrm{~g}, 1.47 \mathrm{mmol})$ and $\mathrm{Cs}_{2} \mathrm{CO}_{3}(2.40 \mathrm{~g}, 7.34 \mathrm{mmol})$ was refluxed in $30 \mathrm{ml}$ acetone for $48 \mathrm{~h}$. After removal of the volatiles under vacuum the crude product was extracted from the residue with $40 \mathrm{ml}$ of $\mathrm{Et}_{2} \mathrm{O}$. The $\mathrm{Et}_{2} \mathrm{O}$ layer was washed with $\mathrm{H}_{2} \mathrm{O}(3 \times 30 \mathrm{ml})$ and dried with $\mathrm{MgSO}_{4}$. Evaporation of the volatiles gave 6 as a slightly yellowish oil $(0.590 \mathrm{~g}, 87 \%) .{ }^{1} \mathrm{H} \mathrm{NMR}\left(\mathrm{CDCl}_{3}\right): \delta_{\mathrm{H}} 6.95(1 \mathrm{H}, \mathrm{t}, \mathrm{J}$ 8.4, PhH), $6.54\left(2 \mathrm{H}, \mathrm{d}, J\right.$ 8.4, PhH), $4.12\left(2 \mathrm{H}, \mathrm{t}, J\right.$ J 5.1, $\left.\mathrm{PhOCH}_{2} \mathrm{CH}_{2}\right), 3.81-3.55$ (20 $\mathrm{H}, \mathrm{m}, \mathrm{PhOCH}_{2} \mathrm{CH}_{2} \mathrm{O}\left(\mathrm{CH}_{2} \mathrm{CH}_{2} \mathrm{O}\right)_{3} \mathrm{Ad}$ and $\left.\mathrm{OCH}_{3}\right), 2.11\left(3 \mathrm{H}, \mathrm{s}, \mathrm{CH}_{2} \mathrm{CHCH}_{2}[\mathrm{Ad}]\right), 1.72$ (6 H, d, J 2.7, $\left.\mathrm{CHCH}_{2} \mathrm{C}[\mathrm{Ad}]\right)$, 1.64-1.54 (6 H, m, $\left.\mathrm{CHCH}_{2} \mathrm{CH}[\mathrm{Ad}]\right) ;{ }^{13} \mathrm{C} \mathrm{NMR}\left(\mathrm{CDCl}_{3}\right)$ : $\delta_{\mathrm{c}} 153.8,137.4,123.8,105.5,72.3,71.5,71.4,71.3,70.9,70.8,70.5,59.5,56.3$, 41.7, 36.7, 30.7; $\mathrm{m} / \mathrm{z}$ (ESI-MS) found: $487.2[\mathrm{M}+\mathrm{Na}]^{+}$, calculated: 464.7 . 
1<smiles>OCCOCCOCCOCCOCCOCCOCCOCCOCCOCCOCCOCCOCCOCCOCCOCCOCCOCCO</smiles><smiles>COc1cccc(OC)c1O</smiles><smiles>COc1cccc(OC)c1OCCOCCOCCOCCOC12CC3CC(CC(C3)C1)C2</smiles>

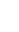

6 $\mathrm{SiO}_{2}, \mathrm{HNO}_{3} \mid \begin{aligned} & \mathrm{CH}_{2} \mathrm{Cl}_{2} \\ & 5 \mathrm{~min}, \mathrm{rt}\end{aligned}$

$P\left(\mathrm{NO}-\mathrm{O}_{\mathrm{O}}\right.$

7
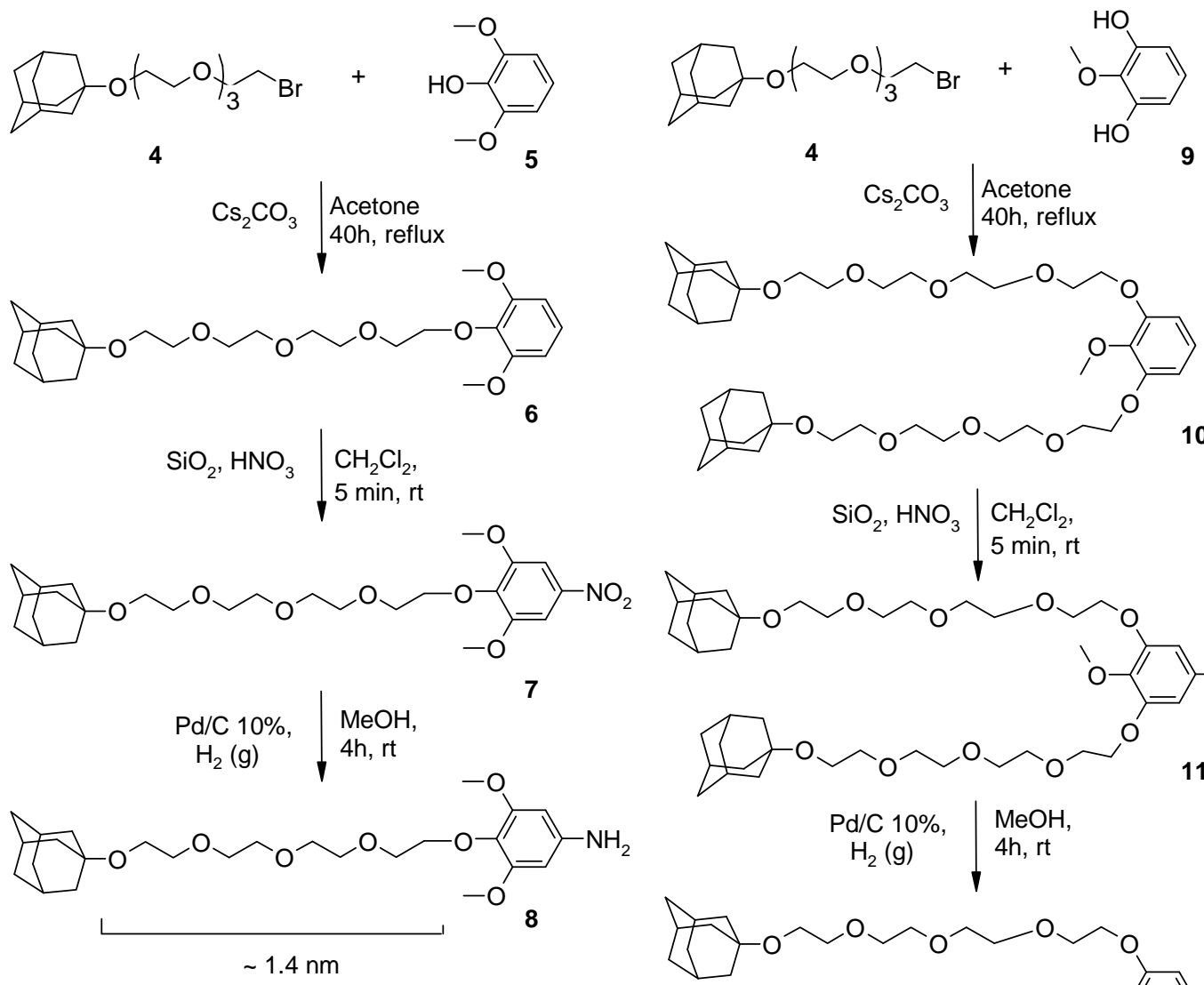

4

9 $\mathrm{Cs}_{2} \mathrm{CO}_{3} \downarrow \begin{aligned} & \text { Acetone } \\ & 40 \mathrm{~h}, \text { reflux }\end{aligned}$<smiles>COc1c(C)cccc1OCCOC[C@H](C)OCCOCCOC12CC3CC(CC(C3)C1)C2</smiles><smiles>CCOCCOCCOCCOCCOC12CC3CC(CC(C3)C1)C2</smiles>
$\mathrm{SiO}_{2}, \mathrm{HNO}_{3} \mid \begin{aligned} & \mathrm{CH}_{2} \mathrm{Cl}_{2}, \\ & 5 \mathrm{~min}, \mathrm{rt}\end{aligned}$<smiles>COc1cc([N+](=O)[O-])cc(OCCOCCOCCOCCOC23CC4CC(CC(C4)C2)C3)c1OCCOCCOCCOCCOC12CC3CC(CC(C3)C1)C2</smiles>

\begin{tabular}{l|l}
$\mathrm{Pd} / \mathrm{C} 10 \%$, & $\mathrm{MeOH}$,
\end{tabular} $\mathrm{H}_{2}(\mathrm{~g}) \downarrow 4 \mathrm{~h}, \mathrm{rt}$<smiles>COc1c(OCCOCCOCCOCCOC23CC4CC(CC(C4)C2)C3)cc(N)cc1OCCOCCOCCOCCOC12CC3CC(CC(C3)C1)C2</smiles>

Scheme 5.5 (note that compounds 8 and 12 refer to Mono-Ad and DiAd, resp, in Scheme 5.1). 
4-[2-(2-\{2-[2-(Adamantan-1-yloxy)ethoxy]ethoxy\}ethoxy)-ethoxy]-3,5-

dimethoxy-1-nitrobenzene (7). A solution of $6(0.590 \mathrm{~g}, 1.27 \mathrm{mmol})$ in $4 \mathrm{ml}$ $\mathrm{CH}_{2} \mathrm{Cl}_{2}$ was added at once to a well stirred suspension of of $\mathrm{SiO}_{2}(0.590 \mathrm{~g})$ and $\mathrm{HNO}_{3}(0.263 \mathrm{ml}, 6.35 \mathrm{mmol})$ in $\mathrm{CH}_{2} \mathrm{Cl}_{2}(3 \mathrm{ml})$. The resulting suspension was stirred for 5 min up on which it was quenched with $3 \mathrm{ml}$ saturated $\mathrm{K}_{2} \mathrm{CO}_{3}$ (aq). The mixture was diluted with $\mathrm{CH}_{2} \mathrm{Cl}_{2}(30 \mathrm{ml})$ and the organic layer was washed with $\mathrm{H}_{2} \mathrm{O}(1 \times 20 \mathrm{ml})$, brine $(2 \times 20 \mathrm{ml})$ and subsequently dried with $\mathrm{MgSO}_{4}$. Evaporation of the volatiles gave pure 7 as a yellow oil $(0.626 \mathrm{~g}, 97 \%)$. ${ }^{1} \mathrm{H}$ NMR $\left(\mathrm{CDCl}_{3}\right): \delta_{\mathrm{H}} 7.50(2 \mathrm{H}, \mathrm{s}, \mathrm{PhH}), 4.24\left(2 \mathrm{H}, \mathrm{t}, J 4.8, \mathrm{PhOCH}_{2} \mathrm{CH}_{2}\right), 3.92\left(6 \mathrm{H}, \mathrm{s}, \mathrm{OCH}_{3}\right)$, $3.81\left(2 \mathrm{H}, \mathrm{t}, J\right.$ 4.8, $\left.\mathrm{PhOCH}_{2} \mathrm{CH}_{2}\right), 3.69-3.56\left(12 \mathrm{H}, \mathrm{m}, \mathrm{PhOCH}_{2} \mathrm{CH}_{2} \mathrm{O}\left(\mathrm{CH}_{2} \mathrm{CH}_{2} \mathrm{O}\right){ }_{3} \mathrm{Ad}\right)$, $2.11\left(3 \mathrm{H}, \mathrm{s}, \mathrm{CH}_{2} \mathrm{CHCH}_{2}[\mathrm{Ad}]\right), 1.73\left(6 \mathrm{H}, \mathrm{d}, J \mathrm{~J}\right.$ 2.7, $\left.\mathrm{CHCH}_{2} \mathrm{C}[\mathrm{Ad}]\right), 1.65-1.55(6 \mathrm{H}, \mathrm{m}$, $\left.\mathrm{CHCH}_{2} \mathrm{CH}[\mathrm{Ad}]\right) ;{ }^{13} \mathrm{C} \mathrm{NMR}\left(\mathrm{CDCl}_{3}\right): \delta_{\mathrm{C}} 153.1,143.6,143.1,101.4,72.7,72.3,71.4-$ 70.5, 59.4, 56.6, 41.6, 36.6, 30.7; $\mathrm{m} / \mathrm{z}$ (ESI-MS) found: $510.3[\mathrm{M}+\mathrm{H}]^{+}$, calculated 509.6 .

\section{4-[2-(2-\{2-[2-(Adamantan-1-yloxy)ethoxy]ethoxy\}ethoxy)-ethoxy]-3,5-}

dimethoxyaniline (8). A suspension of $7(0.626 \mathrm{~g}, 1.23 \mathrm{mmol})$ and a catalytical amount of $\mathrm{Pd} / \mathrm{C}(10 \%)$ was stirred under 1 bar $\mathrm{H}_{2}(\mathrm{~g})$ atmosphere in $10 \mathrm{ml}$ $\mathrm{CH}_{3} \mathrm{OH}$ for $4 \mathrm{~h}$. The suspension was filtered over celite and the volatiles were evaporated under reduced pressure giving the product 8 as a colorless oil $(0.540 \mathrm{~g}, 92 \%)$. The product was pure enough to be used in the subsequent reaction. A small fraction was subjected to column chromatography (eluent $\left.\mathrm{CH}_{2} \mathrm{Cl}_{2} / \mathrm{MeOH}, 14 / 1\right)$ yielding pure $8(0.054 \mathrm{~g}, 59 \%)$ as a colorless oil; ${ }^{1} \mathrm{H} \mathrm{NMR}$ $\left(\mathrm{CDCl}_{3}\right): \delta_{\mathrm{H}} 5.91(2 \mathrm{H}, \mathrm{s}, \mathrm{PhH}), 4.03\left(2 \mathrm{H}, \mathrm{t}, \mathrm{J}\right.$ 5.4, $\left.\mathrm{PhOCH}_{2} \mathrm{CH}_{2}\right), 3.78-3.56(12 \mathrm{H}, \mathrm{m}$, $\mathrm{PhOCH}_{2} \mathrm{CH}_{2} \mathrm{O}\left(\mathrm{CH}_{2} \mathrm{CH}_{2} \mathrm{O}\right)_{3} \mathrm{Ad}$ and $\left.\mathrm{OCH}_{3}\right), 2.12\left(3 \mathrm{H}, \mathrm{s}, \mathrm{CH}_{2} \mathrm{CHCH}_{2}[\mathrm{Ad}]\right), 1.73(6 \mathrm{H}, \mathrm{d}$,

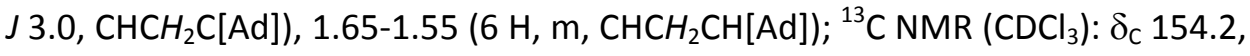
143.1, 130.0, 93.0, 72.6, 72.4, 71.5, 70.5, 59.4, 56.3, 56.2, 56.1, 41.8, 36.6, 30.7; $\mathrm{m} / \mathrm{z}$ (ESI-MS) found: $480.2[\mathrm{M}+\mathrm{H}]^{+}$, calculated: 479.6 .

\section{1,3-Bis[2-(2-\{2-[2-(adamantan-1-yloxy)ethoxy]ethoxy\}ethoxy)-ethoxy]-2-}

methoxybenzene (10). Similar procedure was followed as described for compound 6. ${ }^{1} \mathrm{H}$ NMR $\left(\mathrm{CDCl}_{3}\right): \delta_{\mathrm{H}} 6.92(1 \mathrm{H}, \mathrm{t}, J$ 8.1, $\mathrm{PhH}), 6.59(2 \mathrm{H}, \mathrm{d}, J$ 5.1, $\mathrm{PhH}), 4.16\left(4 \mathrm{H}, \mathrm{t}, \mathrm{J}\right.$ 4.8, $\left.\mathrm{PhOCH}_{2} \mathrm{CH}_{2}\right), 3.86\left(4 \mathrm{H}, \mathrm{t}, \mathrm{J}\right.$ 5.1, $\left.\mathrm{PhOCH}_{2} \mathrm{CH}_{2}\right), 3.38(3 \mathrm{H}, \mathrm{s}$, $\left.\mathrm{OCH}_{3}\right), 3.75-3.65\left(16 \mathrm{H}, \mathrm{m}, \mathrm{PhOCH}_{2} \mathrm{CH}_{2} \mathrm{O}\left(\mathrm{CH}_{2} \mathrm{CH}_{2} \mathrm{O}\right)_{2}\right), 3.59-3.57(8 \mathrm{H}, \mathrm{m}$, 
$\left.\mathrm{CH}_{2} \mathrm{CH}_{2} \mathrm{OAd}\right), 2.13\left(6 \mathrm{H}, \mathrm{s}, \mathrm{CH}_{2} \mathrm{CHCH}_{2}[\mathrm{Ad}]\right), 1.73\left(12 \mathrm{H}, \mathrm{d}, \mathrm{J} 3.0, \mathrm{CHCH}_{2} \mathrm{C}[\mathrm{Ad}]\right)$, 1.65-1.55 (12 H, m, $\left.\mathrm{CHCH}_{2} \mathrm{CH}[\mathrm{Ad}]\right)$.

\section{3,5-Bis[2-(2-\{2-[2-(adamantan-1-yloxy)ethoxy]ethoxy\}ethoxy)-ethoxy]-4-} methoxy-1-nitrobenzene (11). A solution of 10 (0.100 g, $0.131 \mathrm{mmol})$ in $1 \mathrm{ml}$ $\mathrm{CH}_{2} \mathrm{Cl}_{2}$ was added at once to a well stirred suspension of of $\mathrm{SiO}_{2}(0.100 \mathrm{~g})$ and $\mathrm{HNO}_{3}(0.027 \mathrm{ml}, 0.657 \mathrm{mmol})$ in $\mathrm{CH}_{2} \mathrm{Cl}_{2}(1 \mathrm{ml})$. The resulting suspension was stirred for 5 min up on which it was quenched with $3 \mathrm{ml}$ saturated $\mathrm{K}_{2} \mathrm{CO}_{3}(\mathrm{aq})$. The mixture was diluted with $\mathrm{CH}_{2} \mathrm{Cl}_{2}(20 \mathrm{ml})$ and the organic layer was washed with $\mathrm{H}_{2} \mathrm{O}(3 \times 20 \mathrm{ml})$ and subsequently dried with $\mathrm{MgSO}_{4}$. Evaporation of the volatiles gave 11 as a yellow oil $(0.099 \mathrm{~g}, 93 \%) ;{ }^{1} \mathrm{H} N M R\left(\mathrm{CDCl}_{3}\right): \delta_{\mathrm{H}} 7.54(2 \mathrm{H}, \mathrm{s}$, $\mathrm{PhH}), 4.23\left(4 \mathrm{H}, \mathrm{t}, J\right.$ 4.8, $\left.\mathrm{PhOCH}_{2} \mathrm{CH}_{2}\right), 3.94\left(3 \mathrm{H}, \mathrm{s}, \mathrm{OCH}_{3}\right), 3.91-3.88(4 \mathrm{H}, \mathrm{t}, J$ 5.7, $\left.\mathrm{PhOCH}_{2} \mathrm{CH}_{2}\right)$, 3.74-3.56 (24 H, m, PhOCH$\left.{ }_{2} \mathrm{CH}_{2} \mathrm{O}\left(\mathrm{CH}_{2} \mathrm{CH}_{2} \mathrm{O}\right)_{3} \mathrm{OAd}\right), 2.13(6 \mathrm{H}, \mathrm{s}$, $\left.\mathrm{CH}_{2} \mathrm{CHCH}_{2}[\mathrm{Ad}]\right), 1.73\left(12 \mathrm{H}, \mathrm{d}, J \mathrm{~J} .7, \mathrm{CHCH}_{2} \mathrm{C}[\mathrm{Ad}]\right), 1.66-1.55$ (12 $\mathrm{H}, \mathrm{m}$, $\left.\mathrm{CHCH}_{2} \mathrm{CH}[\mathrm{Ad}]\right) ;{ }^{13} \mathrm{C} \mathrm{NMR}\left(\mathrm{CDCl}_{3}\right): \delta_{\mathrm{C}} 152.3,145.1,143.2,143.1,103.5,72.3$, 71.4-70.8, 69.6, 69.4, 59.4, 41.6, 36.6, 30.7; m/z (ESI-MS) found: $806.4[\mathrm{M}]^{+}$, calculated: 806.0 .

\section{3,5-Bis[2-(2-\{2-[2-(adamantan-1-yloxy)ethoxy]ethoxy\}ethoxy)-ethoxy]-4-}

methoxyaniline (12). A suspension of $12(0.099 \mathrm{~g}, 0.123 \mathrm{mmol})$ and a catalytical amount of $\mathrm{Pd} / \mathrm{C}(10 \%)$ was stirred under 1 bar $\mathrm{H}_{2}(\mathrm{~g})$ atmosphere in $5 \mathrm{ml} \mathrm{CH} \mathrm{CH}_{3} \mathrm{OH}$ for $4 \mathrm{~h}$. The suspension was filtered over celite and the volatiles were evaporated under reduced pressure giving the product $\mathbf{1 2}$ as a slightly colored oil $(0.088 \mathrm{~g}, 93 \%) .{ }^{1} \mathrm{H}$ NMR $\left(\mathrm{CDCl}_{3}\right): \delta_{\mathrm{H}} 5.91(2 \mathrm{H}, \mathrm{s}, \mathrm{PhH}), 4.03(4 \mathrm{H}, \mathrm{t}, \mathrm{J} 4.8$, $\mathrm{PhOCH}_{2} \mathrm{CH}_{2}$ ), 3.81-3.56 (31 $\left.\mathrm{H}, \mathrm{m}, \mathrm{PhOCH}_{2} \mathrm{CH}_{2} \mathrm{O}\left(\mathrm{CH}_{2} \mathrm{CH}_{2} \mathrm{O}\right)_{3} \mathrm{OAd}+\mathrm{OCH}_{3}\right), 2.13$ (6 $\left.\mathrm{H}, \mathrm{s}, \mathrm{CH}_{2} \mathrm{CHCH}_{2}[\mathrm{Ad}]\right), 1.73\left(12 \mathrm{H}, \mathrm{d}, J \mathrm{~J}\right.$ 2.7, $\left.\mathrm{CHCH}_{2} \mathrm{C}[\mathrm{Ad}]\right), 1.66-1.55(12 \mathrm{H}, \mathrm{m}$, $\left.\mathrm{CHCH}_{2} \mathrm{CH}[\mathrm{Ad}]\right) ; \mathrm{m} / z$ (ESI-MS) found: $776.3[\mathrm{M}]^{+}$, calculated: 776.1.

\section{1,2,3-Tris[2-(2-\{2-[2-(adamantan-1-yloxy)ethoxy]ethoxy\}ethoxy)-}

ethoxy]benzene (14). A suspension of pyrogallol 13 (3.00 g, $8.64 \mathrm{mmol}), 4$ $(0.363 \mathrm{~g}, 2.88 \mathrm{mmol})$ and $\mathrm{K}_{2} \mathrm{CO}_{3}(1.20 \mathrm{~g}, 8.64 \mathrm{mmol})$ was refluxed in $30 \mathrm{ml}$ acetone for $24 \mathrm{~h}$. Were after the volatiles were removed under vacuum and the crude product was extracted from the residue with $40 \mathrm{ml}$ of $\mathrm{Et}_{2} \mathrm{O}$. $\mathrm{The} \mathrm{Et}_{2} \mathrm{O}$ layer was washed with $\mathrm{H}_{2} \mathrm{O}(3 \times 30 \mathrm{ml})$ and dried with $\mathrm{MgSO}_{4}$. Evaporation of 
the volatiles gave 6 as a slightly colored oil $(2.5 \mathrm{~g}, 82 \%)$ which was pure enough to be used in the subsequent reactions. ${ }^{1} \mathrm{H}$ NMR $(\mathrm{ppm}): \delta=6.91(1 \mathrm{H}, \mathrm{t}, J$ 8.4, $\mathrm{PhH}), 6.58(2 \mathrm{H}, \mathrm{d}, J$ 8.4, $\mathrm{PhH}), 4.23\left(2 \mathrm{H}, \mathrm{t}, \mathrm{J}\right.$ 4.2, $\left.\mathrm{PhOCH}_{2} \mathrm{CH}_{2}-\right)$, 4.19-4.10 (4 H, m, PhOCH $\left.\mathrm{CH}_{2}-\right)$, 3.88-3.56 (42 H, m, PhOCH $\left.\mathrm{CH}_{2} \mathrm{O}\left(\mathrm{CH}_{2} \mathrm{CH}_{2} \mathrm{O}\right)_{3} \mathrm{Ad}\right), 2.13(9 \mathrm{H}, \mathrm{s}$, $\left.\mathrm{CH}_{2} \mathrm{CHCH}_{2}[\mathrm{Ad}]\right), 1.74\left(18 \mathrm{H}, \mathrm{s}, \mathrm{CHCH}_{2} \mathrm{C}[\mathrm{Ad}]\right), 1.65-1.55\left(18 \mathrm{H}, \mathrm{t}, \mathrm{CHCH}_{2} \mathrm{CH}[\mathrm{Ad}]\right)$. ${ }^{13} \mathrm{C} \mathrm{NMR}(\mathrm{ppm}): \delta=152.8,138.5,123.5,107.9,72.3,71.2-68.7,59.2,41.4,36.4$, 30.4. $\mathrm{m} / z$ ESP-MS found: 1079.5 [M+Na] $^{+}$, calculated: $1079.7 . \mathrm{m} / z$ MALDI-TOFMS found: $1079.2[\mathrm{M}+\mathrm{Na}]^{+}$, calculated: 1079.7.

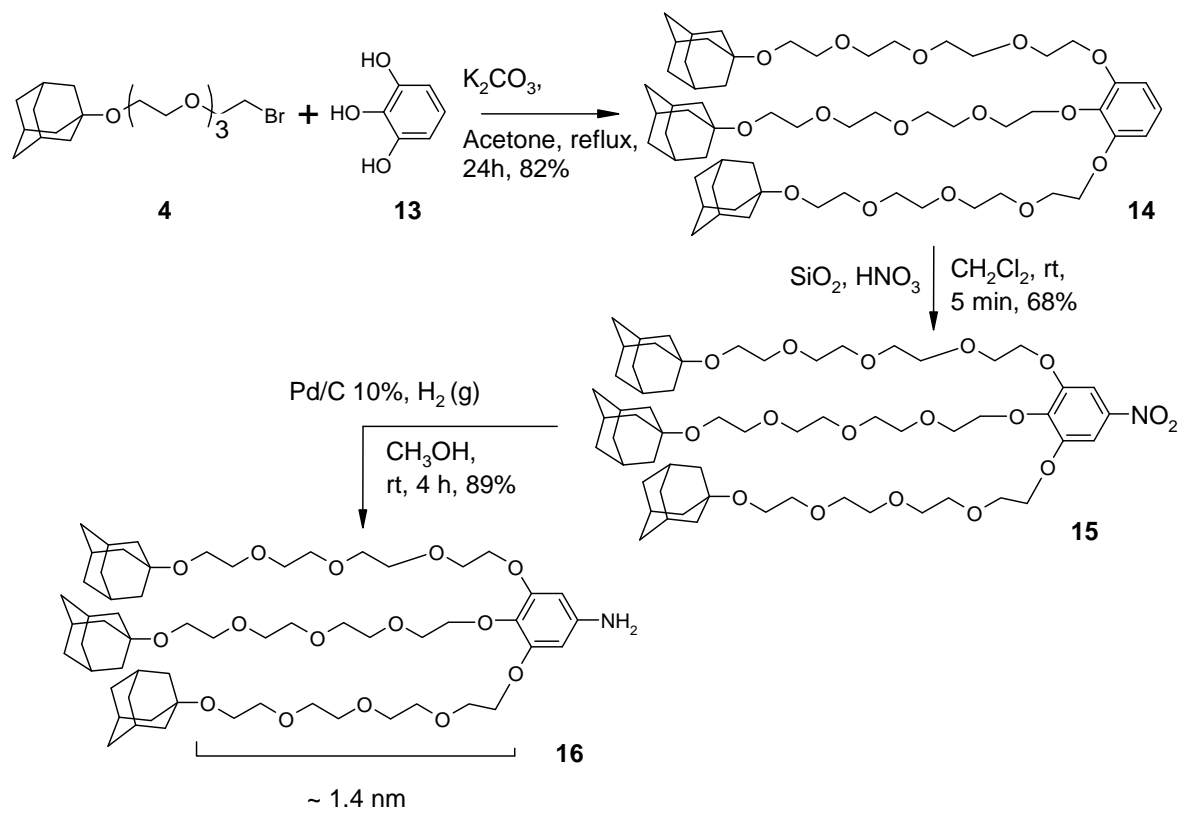

Scheme 5.6 (note that compound 16 refers to Tri-Ad in Scheme 5.1).

\section{3,4,5-Tris[2-(2-\{2-[2-(adamantan-1-yloxy)ethoxy]ethoxy\}ethoxy)-}

ethoxy]phenylnitrate (15). A solution of 14 (0.400 g, $0.38 \mathrm{mmol})$ in $3 \mathrm{ml} \mathrm{CH} \mathrm{Cl}_{2}$ was added at once to a well stirred suspension of $0.400 \mathrm{~g}$ of $\mathrm{SiO}_{2}$ and $\mathrm{HNO}_{3}$ ( $0.08 \mathrm{ml}, 1.89 \mathrm{mmol})$ in $1 \mathrm{ml} \mathrm{CH} \mathrm{Cl}_{2}$. The resulting suspension was stirred for 5 min up on which it was quenched with $3 \mathrm{ml}$ saturated $\mathrm{K}_{2} \mathrm{CO}_{3}$. The solids were filtered off and the filtrate was diluted with $10 \mathrm{ml} \mathrm{CH} \mathrm{Cl}_{2}$. The organic layer was washed with $\mathrm{H}_{2} \mathrm{O}(3 \times 10 \mathrm{ml})$ and dried with $\mathrm{MgSO}_{4}$. Evaporation of the volatiles gave 15 as an oil which was subjected to column chromatography (eluent 
$\left.\mathrm{CH}_{2} \mathrm{Cl}_{2} / \mathrm{MeOH}, 9 / 1\right)$ yielding $15(0.284 \mathrm{~g}, 68 \%)$ as a colorless oil. ${ }^{1} \mathrm{H}$ NMR (ppm): $\delta=7.54(2 \mathrm{H}, \mathrm{s}, \mathrm{PhH}), 4.27\left(2 \mathrm{H}, \mathrm{t}, J 5.1, \mathrm{PhOCH}_{2} \mathrm{CH}_{2}-\right), 4.22(4 \mathrm{H}, \mathrm{t}, J 4.8$, $\left.\mathrm{PhOCH}_{2} \mathrm{CH}_{2}\right), 3.88\left(4 \mathrm{H}, \mathrm{t}, J\right.$ 4.2, $\left.\mathrm{PhOCH}_{2} \mathrm{CH}_{2}\right), 3.79\left(2 \mathrm{H}, \mathrm{t}, J\right.$ 2.4, $\left.\mathrm{PhOCH}_{2} \mathrm{CH}_{2}\right)$, 3.72-3.58 (36 H, m, - $\left.\mathrm{OCH}_{2} \mathrm{CH}_{2} \mathrm{O}-\right), 2,13\left(9 \mathrm{H}, \mathrm{s}, \mathrm{CH}_{2} \mathrm{CHCH}_{2}[\mathrm{Ad}]\right), 1.73(18 \mathrm{H}, \mathrm{s}$, $\left.\mathrm{CHCH}_{2} \mathrm{C}[\mathrm{Ad}]\right), 1.66-1.55\left(18 \mathrm{H}, \mathrm{m}, \mathrm{CHCH}_{2} \mathrm{CH}[\mathrm{Ad}]\right) .{ }^{13} \mathrm{C} \mathrm{NMR}$ (ppm): $\delta=152.4$, 144.3, 143.2, 103.4, 72.9-69.3, 59.6-59.2, 41.6, 36.6, 30.6. $\mathrm{m} / \mathrm{z}$ ESP-MS found: $1102.5[\mathrm{M}]^{+}$, calculated: $1102.4 \mathrm{~m} / z$ MALDI-TOF MS found: $1124.6[\mathrm{M}+\mathrm{Na}]^{+}$, calculated. 1125.4 .

\section{3,4,5-Tris[2-(2-\{2-[2-(adamantan-1-yloxy)ethoxy]ethoxy\}ethoxy)-}

ethoxy]phenylamine (16). A suspension of $15(0.220 \mathrm{~g}, 0.20 \mathrm{mmol})$ and a catalytical amount of $\mathrm{Pd} / \mathrm{C}(10 \%)$ was stirred under 1 bar $\mathrm{H}_{2}(\mathrm{~g})$ atmosphere in 3 $\mathrm{ml}$ methanol for $4 \mathrm{~h}$. The suspension was filtered over celite and the volatiles were evaporated giving the product as a colorless oil $(0.19 \mathrm{~g}, 89 \%)$. The product was pure enough to be used in the subsequent reaction. ${ }^{1} \mathrm{H}$ NMR (ppm): $\delta=$ 6.32 (2 H, s, PhH), 4.12-4.02 (6 H, m, PhOCH $\left.\mathrm{CH}_{2}-\right)$, 3.84-3.73 (6 H, PhOCH $\left.\mathrm{CH}_{2}\right)$, 3.69-3.56 (36 H, m, - $\left.\mathrm{OCH}_{2} \mathrm{CH}_{2} \mathrm{O}-\right), 2,12\left(9 \mathrm{H}, \mathrm{s}, \mathrm{CH}_{2} \mathrm{CHCH}_{2}[\mathrm{Ad}]\right), 1.72(18 \mathrm{H}, \mathrm{s}$, $\left.\mathrm{CHCH}_{2} \mathrm{C}[\mathrm{Ad}]\right), 1.64-1.54\left(18 \mathrm{H}, \mathrm{m}, \mathrm{CHCH}_{2} \mathrm{CH}[\mathrm{Ad}]\right) .{ }^{13} \mathrm{C} \mathrm{NMR}$ (ppm): $\delta=153.2$, 136.2, 108.0, 99.6, 72.8-69.1, 59.4, 41.6, 36.6, 30.6. $\mathrm{m} / \mathrm{z}$ MALDI-TOF MS found: $1072.7[\mathrm{M}+\mathrm{H}]^{+}$, calculated: 1072.7 .

The contour length of the PEG chains for these molecules is around $1.4 \mathrm{~nm}$ which ensures the possibility of trivalent bonding to the CD SAM surface. (The molecular weight of one PEG monomer is 44 and the calculated length of one monomer is $3.64 \AA$ (in a linear form with COC and CCO angles of 113 and 111 degrees respectively). 

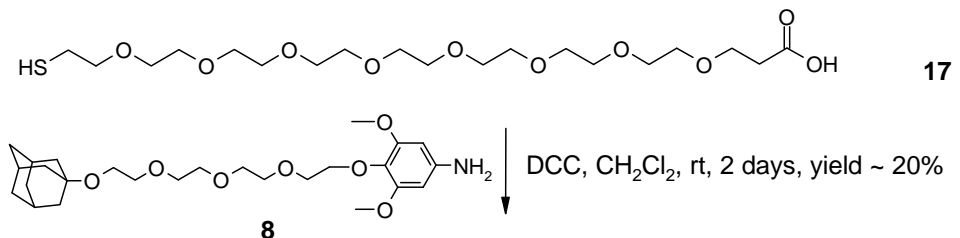

8

19

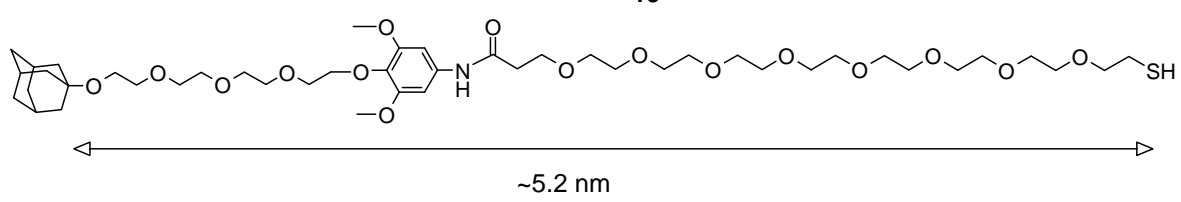

Scheme 5.7 (note that compound 19 refers to Ad-EG13-SH as given in Scheme 5.1).

4-[2-(2-\{2-[2-(Adamantan-1-yloxy)ethoxy]ethoxy\}ethoxy)-ethoxy]-3,5dimethoxyphenyl O-(carboxyethyl)-O'-2-mercaptoethyl)-heptaethylene glycol amide (19). A solution of $8(0.108 \mathrm{~g}, 0.225 \mathrm{mmol}), 17(0.103 \mathrm{~g}, 0.225 \mathrm{mmol})$ and dicyclohexylcarbodiimide (DCC) $(0.47 \mathrm{~g}, 0.225 \mathrm{mmol})$ was stirred at $\mathrm{rt}$ in $5 \mathrm{ml}$ $\mathrm{CH}_{2} \mathrm{Cl}_{2}$ for $48 \mathrm{~h}$. Hereafter the white precipitate was filtered off over celite. Evaporation of the volatiles gave the crude product as an oil which was subjected to column chromatography (eluent $\mathrm{CH}_{2} \mathrm{Cl}_{2} / \mathrm{MeOH}, 9 / 1$ ) yielding partly dimerized $19(0.042 \mathrm{~g}, 20 \%)$ as a colorless oil; ${ }^{1} \mathrm{H}$ NMR $\left(\mathrm{CDCl}_{3}\right): \delta_{\mathrm{H}} 8.52(1 \mathrm{H}, \mathrm{s}$, $\mathrm{NH}), 6.89(1 \mathrm{H}, \mathrm{s}, \mathrm{PhH}), 4.09\left(2 \mathrm{H}, \mathrm{t}, J 5.7, \mathrm{PhOCH}_{2} \mathrm{CH}_{2}-\right), 3.82\left(6 \mathrm{H}, \mathrm{s}, \mathrm{OCH}_{3}-\right)$, 3.85-3.56 (46 H, m, OCH $\left.\mathrm{CH}_{2} \mathrm{O}+\mathrm{PhOCH}_{2} \mathrm{CH}_{2}+\mathrm{OCH}_{2} \mathrm{CH}_{2} \mathrm{SH}+\mathrm{NHC}(\mathrm{O}) \mathrm{CH}_{2} \mathrm{CH}_{2} \mathrm{O}\right)$, $2.88\left(1.7 \mathrm{H}, \mathrm{t}, J\right.$ J 6.6, $\left.-\mathrm{CH}_{2} \mathrm{SSCH}_{2}-\right)$, 2.72-2.61 (3.2 H, m, $\left.-\mathrm{CH}_{2} \mathrm{SH}+\mathrm{C}(\mathrm{O}) \mathrm{CH}_{2} \mathrm{CH}_{2} \mathrm{O}\right)$, 2.17-2.12 (3H, m, $\left.\mathrm{CH}_{2} \mathrm{CHCH}_{2}[\mathrm{Ad}]\right), 1.78-1.71\left(6 \mathrm{H}, \mathrm{m}, \mathrm{CHCH}_{2} \mathrm{C}[\mathrm{Ad}]\right), 1.71-1.56$ (6 $\left.\mathrm{H}, \mathrm{m}, \mathrm{CHCH}_{2} \mathrm{CH}[\mathrm{Ad}]\right) ; \delta_{\mathrm{c}} 153.8,137.4,123.8,105.5,72.3,71.6-71.3,70.9-70.5$, 59.6-59.3, 56.5-56.0, 41.7, 36.6, 30.7; $\mathrm{m} / \mathrm{z}$ (ESI-MS) found: $920.4[\mathrm{M}+\mathrm{H}]^{+}$, calculated $919.5[\mathrm{M}]^{+}$.

(2-(2-(2-(2-(Adamantyl-1-oxy)ethoxy)ethoxy)ethoxy)ethanethiol (Ad-EG4-SH). Halide 4 (3 mmol) was dissolved in $5 \mathrm{ml}$ ethanol in a $25 \mathrm{ml}$ flask. Sodium thiosulfate ( $3 \mathrm{mmol}$ ) was dissolved in $5 \mathrm{ml}$ of water, and the resulting solution was added to the ethanol one. The mixture was heated at reflux for $18 \mathrm{~h}$. Hydrolysis was carried out by adding $3 \mathrm{ml}$ of $10 \%$ aqueous sulphuric acid and refluxing for $7 \mathrm{~h}$. The title thiol product was extracted with ether, washed with 
water and dried with potassium sulphate, filtered, and freed from solvent. ${ }^{1} \mathrm{H}$ NMR $\left(\mathrm{CDCl}_{3}\right): \delta_{\mathrm{H}}=3.85-3.57\left(\mathrm{~m}, 14 \mathrm{H} ; \mathrm{CH}_{2} \mathrm{CH}_{2} \mathrm{O}+\mathrm{CH}_{2} \mathrm{CH}_{2} \mathrm{SH}\right), 2.75$ (3 H; $\mathrm{CH}_{2} \mathrm{CH}_{2} \mathrm{SH}$ ), 2.15 (m, $\left.3 \mathrm{H} ; \mathrm{CH}_{2} \mathrm{CHCH}_{2}[\mathrm{Ad}]\right), 1.76-1.75$ (m, $\left.6 \mathrm{H} ; \mathrm{CHCH}_{2} \mathrm{C}[\mathrm{Ad}]\right), 1.64-$ $1.62\left(\mathrm{~m}, 6 \mathrm{H} ; \mathrm{CHCH}_{2} \mathrm{CH}[\mathrm{Ad}]\right) . \mathrm{m} / z$ (ESI-MS) found: $345.2[\mathrm{M}+\mathrm{H}]^{+}$, calculated $344.5[\mathrm{M}]^{+}$

\section{Cyclodextrin monolayer}

All glassware was cleaned to remove organic contamination by using piranha solution (3:1 mixture of sulfuric acid and hydrogen peroxide) and thoroughly rinsed with $M Q$ water and ethanol.

Gold coated (200 nm thickness) glass squares were purchased from Arrandee (Arrandee, Germany). After rinsing with chloroform they were annealed under a high purity hydrogen flame for about $5 \mathrm{~min}$ and allowed to cool down. CD adsorbate (1-2 mg) was dissolved in a 3:2 solution of chloroform and ethanol (total volume $50 \mathrm{ml}$ ). The gold substrates were immersed in fresh piranha solution for 10-20 s to remove any organic contamination, rinsed with $\mathrm{MQ}$ water and ethanol and immediately put into the CD solution. The samples were kept in this solution overnight at 60 oC, rinsed sequentially with dichloromethane, ethanol and $\mathrm{MQ}$ water to remove any excess of adsorbed material and used immediately in the force spectroscopy experiments.

\section{Functionalization of AFM tips}

Gold coated V-shaped AFM cantilevers with pyramidal tips (Veeco, Digital Instruments, USA) were cleaned in piranha solution for $10 \mathrm{~s}$, carefully rinsed with MQ water and ethanol. In the case of Ad-EG4-SH functionalization the cantilevers were immersed in an ethanol solution of 0.5\% Ad-EG4-SH and 99.5\% 2-mercaptoethanol (1 $\mathrm{mM}$ total thiol concentration) overnight. For the AdEG13-SH functionalization the cantilevers were immersed in an ethanol solution 25\% Ad-EG13-SH and 75\% 2-mercaptoethanol (1 mM total thiol concentration) overnight. Finally the cantilevers were rinsed with ethanol and blown dry in a stream of nitrogen. For the PEG 3000 functionalization the cantilevers were cleaned as described before and immersed in an ethanol solution of cystamine hydrochloride $(1 \mathrm{mM})$ for $3 \mathrm{~h}$. After rinsing with ethanol and dichloromethane (DCM), the amine terminated cantilevers were incubated for $2 \mathrm{~h}$ in a 100:1 DCM/ N,N-diisopropylethylamine (DIPEA) solution with PEG 3000 linker (NHSPEG-NHS, $5 \mathrm{mg} / \mathrm{ml}, \sim 1 \mathrm{mM}$ ) and rinsed with dichloromethane. Finally they were 
immersed for $2 \mathrm{~h}$ in a solution of amino terminated mono- di- or triadamantane guest (1 mM) in 100:1 DCM/DIPEA, rinsed with DCM and blown dry in a stream of nitrogen.

\section{AFM force spectroscopy measurements}

All force measurements were performed with a commercial Multimode Picoforce SPM (Veeco, Digital Instruments, USA) using a liquid cell (Veeco) and stock PBS buffer (B. Braun Melsungen AG, Germany). The spring constants of the cantilevers were calibrated using the built-in thermal tune software. Forcedistance curves were acquired by approaching and retracting the tip at speeds ranging $10-10^{3} \mathrm{~nm} / \mathrm{s}$. The tip was laterally displaced over the substrate between each approach-retraction cycle, covering $1 \mu^{2}$, and the maximum force applied to the surface was kept under $500 \mathrm{pN}$. Two different cantilevers were used for each guest. We could obtain up to 5000 curves per cantilever, limited by the appearance of high adhesion between the tip and surface and a large decrease in the chance of observing polymer stretching. This we attribute to deterioration of the monolayers due to the repeated contact or adsorption of contamination on the tip.

\section{Data analysis}

We selected relevant force curves using our own plug-in script developed for Hooke. $^{33}$ Each curve was examined for sudden changes in force (rupture events), then the data prior to the last rupture (the unbinding event happening farthest away from the surface) were fitted using a WLC model with fixed persistence length $3.5 \AA$. We estimate the thermal noise from the standard deviation $\left(\sigma_{N C}\right)$ of points in the non-contact area (green triangles in Figure 5.7). The quality of the fit is assessed by comparing this thermal noise with the average distance from data to fit $\left(d_{D-F}\right)$ in the polymer stretch region (blue dots in Figure 5.7);

$$
d_{D-F}=\frac{1}{n} \sum_{k=1}^{n} d_{k}=\frac{1}{n} \sum_{k=1}^{n}\left|f_{k}-f i t\left(f_{k}\right)\right|
$$

with $f_{k}$ the force measured for each data point in the polymer stretch region, $f i t\left(f_{k}\right)$ the force value obtained from the WLC fit at that same abscissa and $n$ the total number of fitted points. 


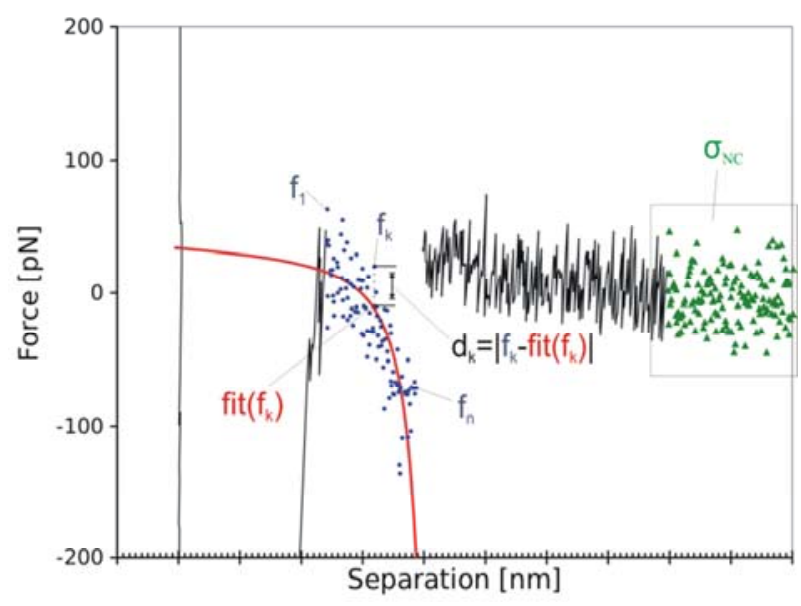

Figure 5.7 Force-curve filtering procedure.

If the ratio $d_{D-F} / \sigma_{N C}$ is close to one, the difference between the fit and data can be explained by thermal noise and the curve is kept for further analysis. Once a curve was accepted for analysis, the slope of the fit at the rupture point was multiplied by the retraction speed to obtain the instantaneous loading rate. The difference between the minimum force value prior to the rupture and the average force value in the following plateau was saved together with this loading rate and the fitted contour length as (rupture force, loading rate, contour length) sets of values for further treatment. Pairs of rupture force and instantaneous loading rate were obtained from the valid events and an estimation of the rupture force and loading rate probability densities was obtained with the help of the statistical package $R^{34}$ by using kernel density estimation with Epanechnikov kernels and a fixed bandwidth of $10 \mathrm{pN}$ in the case of force or an automatically selected bandwidth (Sheather and Jones algorithm $)^{35}$ in the case of loading rate and contour length. The densities obtained from analysis of tips connected to mono-, di- and trivalent guests at different retracting speeds can be seen respectively in Figure 5.8, Figure 5.9 and Figure 5.10. 

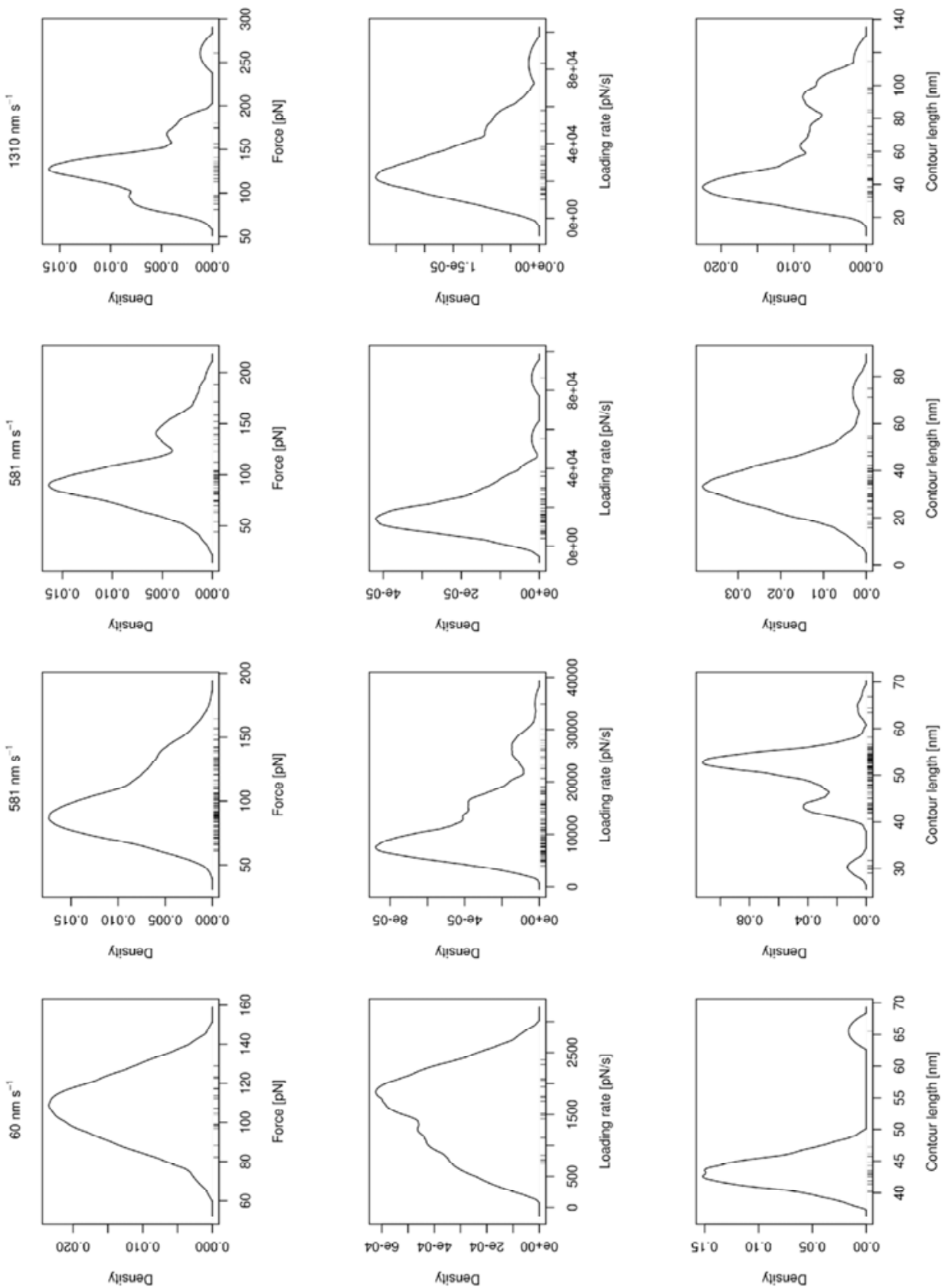

Figure 5.8 Monovalent guest, PEG $\mathbf{3 0 0 0}$ linker. Density of probability of rupture force (top row), loading rate (middle row) and contour length of the linker (bottom row) for different probe retraction speeds (columns). 

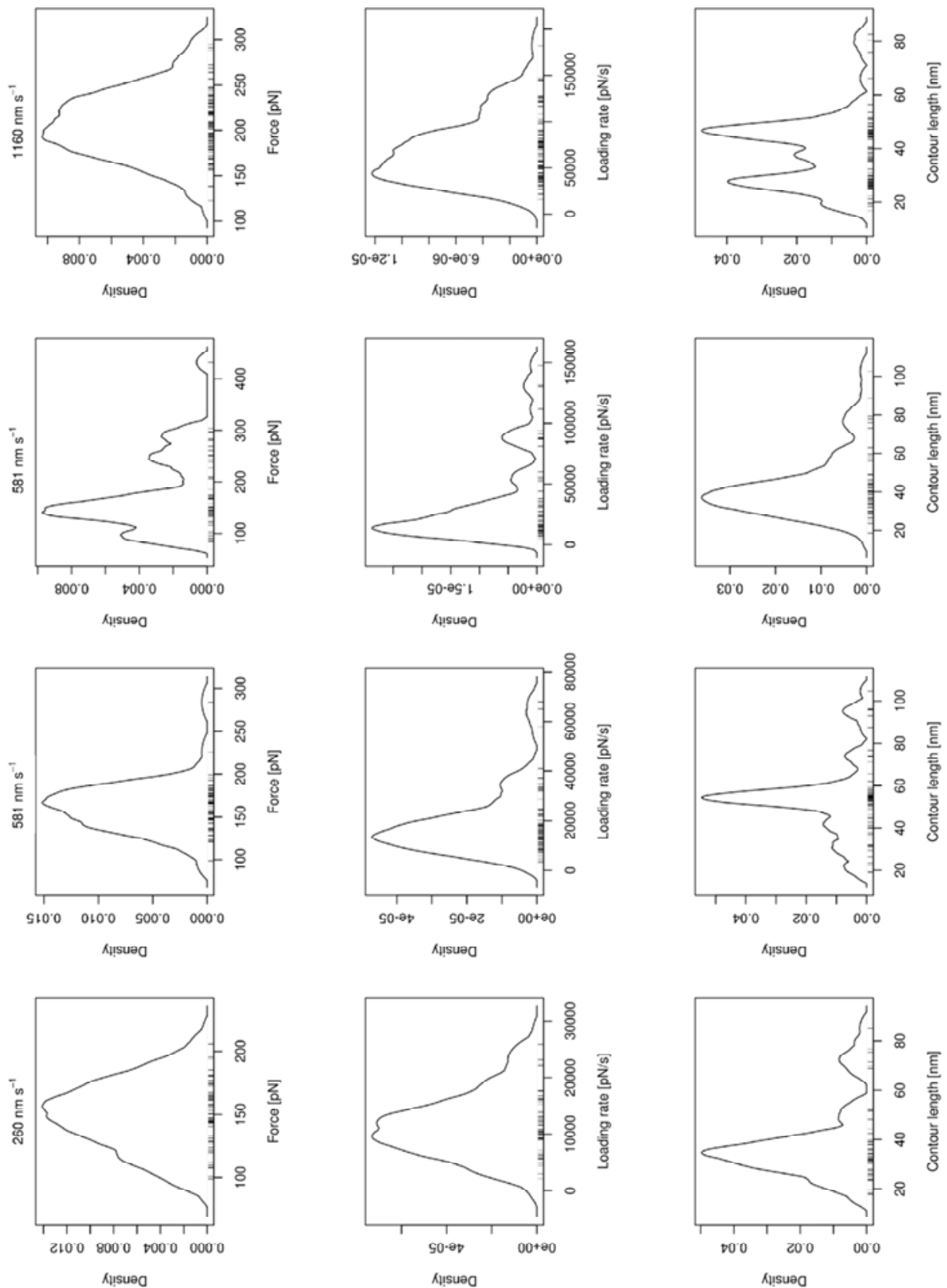

Figure 5.9 Divalent guest, PEG 3000 linker. Density of probability of rupture force (top row), loading rate (middle row) and contour length of the linker (bottom row) for different probe retraction speeds (columns). 

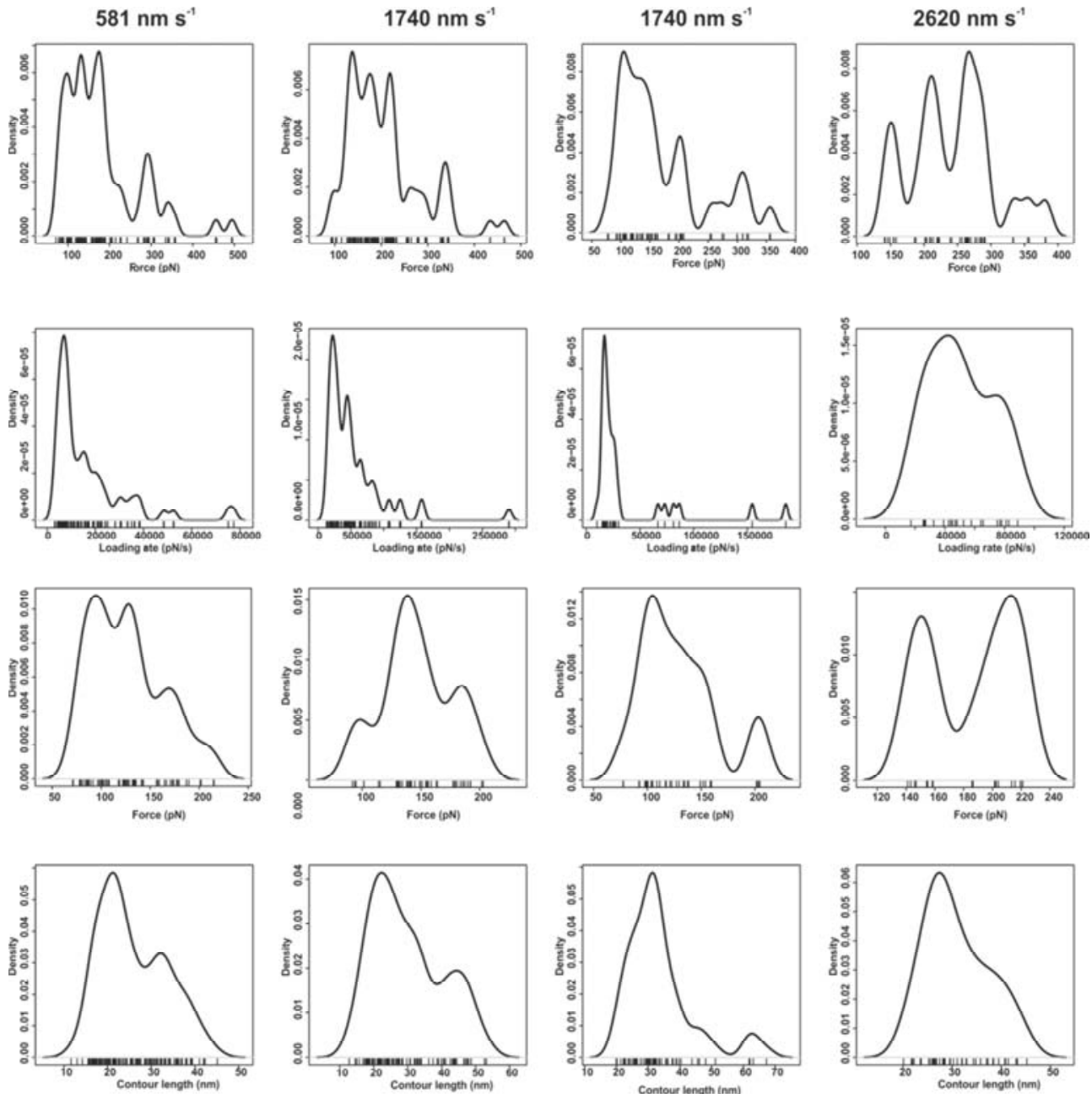

Figure 5.10 Trivalent guest, PEG $\mathbf{3 0 0 0}$ linker. Density of probability of rupture force (top row), loading rate (2nd row), rupture force corresponding the main loading rate peak (3rd row, see Figure 5.4) and contour length of the linker (bottom row) for different probe retraction speeds (columns). 


\subsection{Appendix A: Thermodynamic equilibrium of monovalent complexes}

A model describing the behaviour of fast rebinding complexes under dynamic loading, developed by Diezemann and Janshoff, ${ }^{27}$ was implemented. The input parameters for this model describe the potential energy of the host-guest interaction (Figure 5.11), and their values were based on previous studies of the cyclodextrin-adamantane complex (Table 5.2). The guest is supposed to perform fast transitions from a bound state to an unbound state (the two potential wells in the figure).

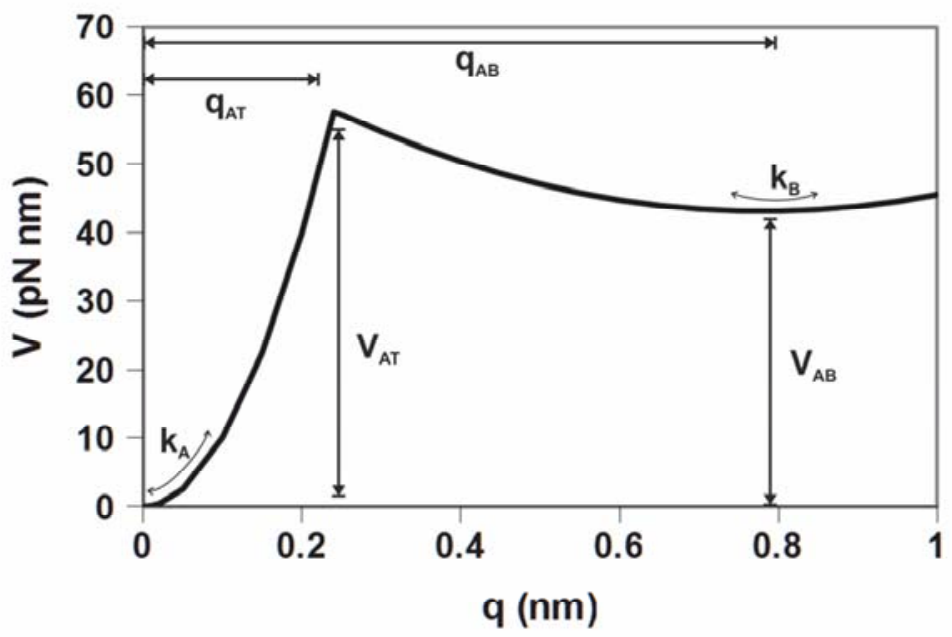

Figure 5.11 Model for the potential of the monovalent host-guest interaction. The horizontal axis represents the reaction coordinate. See values and explanation of parameters in Table 5.2.

The mean rupture force at several loading rates was calculated using this model. A comparison between the results of this model and the rupture forces of monovalent adamantly guests measured by FS is shown in Figure 5.12. 
Table 5.2 Values for the parameters presented in Figure 5.11.

\begin{tabular}{|c|c|l|}
\hline $\begin{array}{c}\text { Parameter } \\
\text { (units) }\end{array}$ & Value & \multicolumn{1}{c|}{ Based on } \\
\hline $\mathbf{k}_{\mathrm{A}}(\mathbf{p N} / \mathbf{n m})$ & 2000 & $\mathrm{k}_{\text {complex } 1250-3000 \mathrm{pN} / \mathrm{nm}^{17}}$ \\
\hline $\mathbf{k}_{\mathrm{B}}(\mathbf{p N} / \mathbf{n m})$ & 100 & Cantilever spring constant \\
\hline $\mathbf{q}_{\mathrm{AB}}(\mathbf{n m})$ & 0.78 & Depth of cyclodextrin cavity \\
\hline $\mathbf{q}_{\mathrm{AT}}(\mathbf{n m})$ & 0.24 & $\begin{array}{l}\text { Fixed by other parameters } \\
\mathrm{x}_{\beta} \text { of di- and trivalent } 0.2-0.3 \mathrm{~nm}\end{array}$ \\
\hline $\mathbf{V}_{\mathbf{A B}}(\mathbf{p N ~} \mathbf{n m})$ & 43.16 & $\Delta G$ of $\beta C D-A d(26 \mathrm{~kJ} /$ mol) \\
\hline $\mathbf{V}_{\mathrm{AT}}(\mathbf{p N} \mathbf{n m})$ & 60.48 & Fixed by other parameters \\
\hline
\end{tabular}

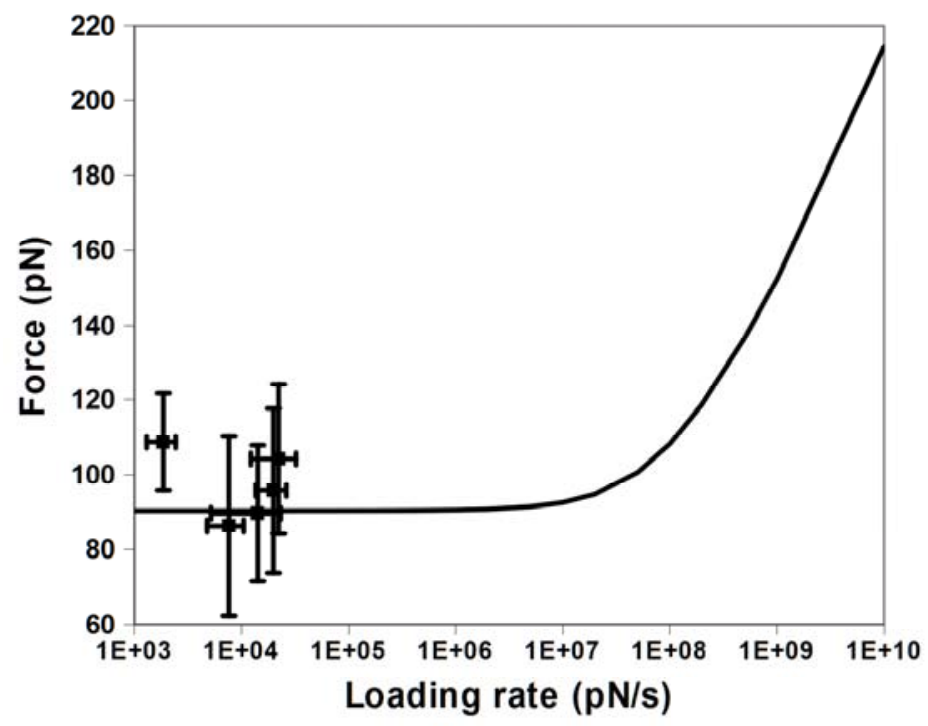

Figure 5.12 Comparison between the experimental values for the rupture force of monovalent guest (symbols) and the predicted mean rupture force using Diezemann model (line). 


\subsection{Appendix B: Effect of the linkers on the stability of the fully- bound trivalent guest}

As it was presented in the main text, the model-estimated off-rate ( $k_{\text {off-Tlestimated) }}$ $\left.=2 \cdot 10^{-4} \mathrm{~s}^{-1}\right)$ is lower than the off-rate obtained from the DFS experiments $k_{\text {off }-T}$ $\left(4 \cdot 10^{-3} \mathrm{~s}^{-1}\right)$. To explain why the model is less accurate in the case of trivalent binding we examine in more detail the geometry of our system. AFM studies of the $\beta C D$ monolayer suggest a hexagonal packing with a lattice constant of 21 $\AA .{ }^{18}$ In the case of a divalent bond, this distance must be bridged by the $8 \mathrm{EG}$ units present in the structure (4 EG per "leg", see Figure 5.13).

The structure of polyethylene glycol in aqueous media has been previously studied, indicating that water molecules can form bridges with the oxygen atoms in the chain when the bonds are in trans-trans-gauche (ttg) configuration. ${ }^{22 a}$ An EG group in ttg conformation has a length of $2.8 \AA$. If the chain is stretched the water bridge is broken and the EG adopt an all-trans (ttt) configuration, with a unitary length of $3.58 \AA$, with an energy penalty $\Delta G_{\text {ttg-stt }}$ of approx. $3 k_{B} T(7.5 \mathrm{~kJ} / \mathrm{mol})$. This is compatible with a situation where our divalent guest is doubly bound and at the same time all the 8 EG groups in the chain connecting the two adamantyl moieties ( $21 \AA$ apart) can be still in the ttg configuration (for a total length of $23 \AA$ ). However, in the case of the fully bound trivalent guest $4 \mathrm{EG}$ units must cover the distance from the centroid of the three occupied cavities to the center of each of those cavities (12.1 $\AA$ ), which is not possible with all the four groups in the ttg configuration (total length of $11.2 \AA$ ). Thus, at least one of the water bridges must be broken (for a total length of $12 \AA$ ) to allow for trivalent complexation.

Our theoretical model suggests an equilibrium constant for the trivalent complex:

$K_{T(\text { model })}=\frac{1}{3} C_{e f f}^{2} K_{M}^{3}=1.310^{12} M^{-1}$

With $C_{\text {eff }}=0.2 \mathrm{M}$ and $K_{M}=4.610^{4} \mathrm{M}^{-1}$, the factor $1 / 3$ accounting for the factor $C_{\text {eff }}^{\prime}$ (see Scheme 5.3).

On the other hand, we can calculate an equilibrium constant based on the offrate obtained from $\mathrm{FS}$ experiments:

$K_{T(F S)}=\frac{k_{\text {onT }}}{k_{\text {off } f(F S)}}=7.510^{10} \mathrm{M}^{-1}$ 
The free energies corresponding to these equilibrium constants are $\Delta G_{T(\text { model })}=$ $69.6 \mathrm{~kJ} / \mathrm{mol}$ and $\Delta G_{T(F S)}=62.6 \mathrm{~kJ} / \mathrm{mol}$. The difference between these energies ( 7 $\mathrm{kJ} / \mathrm{mol}$ ) is very close to the predicted energy cost of elongating one of the EG groups in the structure $(7.5 \mathrm{~kJ} / \mathrm{mol}) .{ }^{22 a}$ This effect is not included in the theoretical model and explains the reduced stability observed in the trivalent FS experiments.

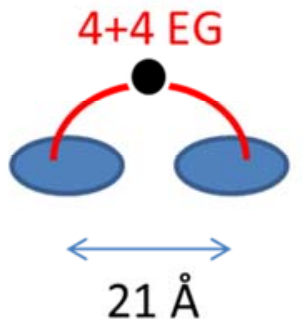

2.6 Å per EG

(4 EG in ttg)

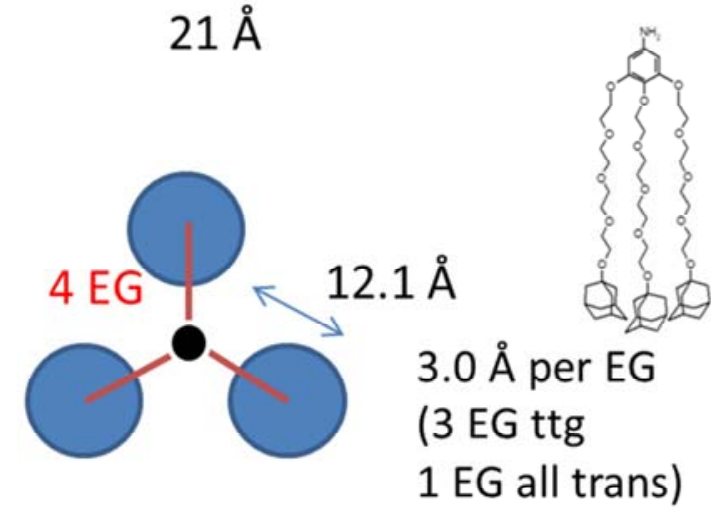

Figure 5.13 Geometrical estimations for the fully-bound divalent ant trivalent complexes.

\subsection{Acknowledgements}

We thank Dr. Henk H. Dam, Deniz Yilmaz and Daniel Florea for the synthesis of the compounds used in this study. 


\subsection{References and notes}

1. (a) Ehrlich, P. H., J. Theor. Biol. 1979, 81, 123-7; (b) Thobhani, S.; Ember, B.; Siriwardena, A.; Boons, G. J., J. Am. Chem. Soc. 2003, 125, 7154-5; (c) Scobie, H. M.; Young, J. A., Curr. Opin. Microbiol. 2005, 8, 106-12.

2. (a) Kiessling, L. L.; Gestwicki, J. E.; Strong, L. E., Angew. Chem. Int. Ed. 2006, 45, 2348-68; (b) Martos, V.; Castreno, P.; Valero, J.; de Mendoza, J., Curr. Opin. Chem. Biol. 2008, 12, 698-706; (c) Sriram, S. M.; Banerjee, R.; Kane, R. S.; Kwon, Y. T., Chem. Biol. 2009, 16, 121-31.

3. (a) Badjic, J. D.; Nelson, A.; Cantrill, S. J.; Turnbull, W. B.; Stoddart, J. F., Acc. Chem. Res. 2005, 38, 723-32; (b) Mulder, A.; Huskens, J.; Reinhoudt, D. N., Org. Biomol. Chem. 2004, 2, 3409-24.

4. (a) Bizzarri, A. R.; Cannistraro, S., Chem. Soc. Rev. 2010, 39, 734-49; (b) Neuman, K. C.; Nagy, A., Nat. Methods 2008, 5, 491-505.

5. Evans, E.; Ritchie, K., Biophys. J. 1997, 72, 1541-55.

6. (a) Merkel, R.; Nassoy, P.; Leung, A.; Ritchie, K.; Evans, E., Nature 1999, 397, 50-3; (b) Hummer, G.; Szabo, A., Acc. Chem. Res. 2005, 38, 504-13; (c) Harris, N. C.; Song, Y.; Kiang, C. H., Phys. Rev. Lett. 2007, 99, 068101; (d) Odorico, M.; Teulon, J. M.; Bessou, T.; Vidaud, C.; Bellanger, L.; Chen, S. W.; Quemeneur, E.; Parot, P.; Pellequer, J. L., Biophys. J. 2007, 93, 645-54.

7. (a) Marszalek, P. E.; Lu, H.; Li, H.; Carrion-Vazquez, M.; Oberhauser, A. F.; Schulten, K.; Fernandez, J. M., Nature 1999, 402, 100-3; (b) Rief, M.; Gautel, M.; Oesterhelt, F.; Fernandez, J. M.; Gaub, H. E., Science 1997, 276, 1109-12.

8. (a) Yu, J.; Malkova, S.; Lyubchenko, Y. L., J. Mol. Biol. 2008, 384, 9921001; (b) Sandal, M.; Valle, F.; Tessari, I.; Mammi, S.; Bergantino, E.; Musiani, F.; Brucale, M.; Bubacco, L.; Samori, B., PLoS Biol 2008, 6, e6.

9. Duman, M.; Pfleger, M.; Zhu, R.; Rankl, C.; Chtcheglova, L. A.; Neundlinger, I.; Bozna, B. L.; Mayer, B.; Salio, M.; Shepherd, D.; Polzella, P.; Moertelmaier, M.; Kada, G.; Ebner, A.; Dieudonne, M.; Schutz, G. J.; Cerundolo, V.; Kienberger, F.; Hinterdorfer, P., Nanotechnology 2010, 21, 115504.

10. Garcia-Manyes, S.; Liang, J.; Szoszkiewicz, R.; Kuo, T.-L.; Fernández, J. M., Nat. Chem. 2009, 1, 236-42.

11. (a) Ratto, T. V.; Rudd, R. E.; Langry, K. C.; Balhorn, R. L.; McElfresh, M. W., Langmuir 2006, 22, 1749-57; (b) Sulchek, T.; Friddle, R. W.; Noy, A., Biophys. J. 2006, 90, 4686-91; (c) Zhang, Y.; Yu, Y.; Jiang, Z.; Xu, H.; Wang, Z.; Zhang, X.; Oda, M.; Ishizuka, T.; Jiang, D.; Chi, L.; Fuchs, H., Langmuir 2009, 25, 6627-32.

12. Ercolani, G.; Schiaffino, L., Angew. Chem. Int. Ed. 2011, 50, 1762-8.

13. Guo, S.; Ray, C.; Kirkpatrick, A.; Lad, N.; Akhremitchev, B. B., Biophys. J. 2008, 95, 3964-76. 
14. Ludden, M. J. W.; Reinhoudt, D. N.; Huskens, J., Chem. Soc. Rev. 2006, 35, 1122-34.

15. Szejtli, J., Chem. Rev. 1998, 98, 1743-54.

16. (a) Mulder, A.; Auletta, T.; Sartori, A.; Del Ciotto, S.; Casnati, A.; Ungaro, R.; Huskens, J.; Reinhoudt, D. N., J. Am. Chem. Soc. 2004, 126, 6627-36; (b) Huskens, J.; Mulder, A.; Auletta, T.; Nijhuis, C. A.; Ludden, M. J.; Reinhoudt, D. N., J. Am. Chem. Soc. 2004, 126, 6784-97.

17. Auletta, T.; de Jong, M. R.; Mulder, A.; van Veggel, F. C.; Huskens, J.; Reinhoudt, D. N.; Zou, S.; Zapotoczny, S.; Schönherr, H.; Vancso, G. J.; Kuipers, L., J. Am. Chem. Soc. 2004, 126, 1577-84.

18. Beulen, M. W.; Bugler, J.; de Jong, M. R.; Lammerink, B.; Huskens, J.; Schönherr, H.; Vancso, G. J.; Boukamp, B. A.; Wieder, H.; Offenhauser, A.; Knoll, W.; van Veggel, F. C.; Reinhoudt, D. N., Chem-Eur. J. 2000, 6, 1176-83.

19. (a) Mulder, A.; Onclin, S.; Peter, M.; Hoogenboom, J. P.; Beijleveld, H.; ter Maat, J.; Garcia-Parajo, M. F.; Ravoo, B. J.; Huskens, J.; van Hulst, N. F.; Reinhoudt, D. N., Small 2005, 1, 242-53; (b) Thompson, D., Langmuir 2007, 23, 8441-51.

20. Hinterdorfer, P.; Kienberger, F.; Raab, A.; Gruber, H. J.; Baumgartner, W.; Kada, G.; Riener, C.; Wielert-Badt, S.; Borken, C.; Schindler, H., Single Mol. 2000, 1, 99-103.

21. Test fits of similar quality were obtained using freely jointed chain (FJC) model. WLC was preferred due to its availability in the employed data analysis tool (Hooke, see ref. 28).

22. (a) Oesterhelt, F.; Rief, M.; Gaub, H. E., New J. Phys. 1999, 1, 6.1-11; (b) Thormann, E.; Hansen, P. L.; Simonsen, A. C.; Mouritsen, O. G., Colloids Surf., B 2006, 53, 149-56.

23. Bell, G. I., Science 1978, 200, 618-27.

24. Schönherr, H.; Beulen, M. W. J.; Bugler, J.; Huskens, J.; van Veggel, F. C. J. M.; Reinhoudt, D. N.; Vancso, G. J., J. Am. Chem. Soc. 2000, 122, 4963-7.

25. Zou, S.; Schönherr, H.; Vancso, G. J., J. Am. Chem. Soc. 2005, 127, 11230-1.

26. Novo, M.; Granadero, D.; Bordello, J.; Al-Soufi, W., J. Incl. Phenom. Macrocycl. Chem. 2010, 11, 173-88.

27. Diezemann, G.; Janshoff, A., J. Chem. Phys. 2008, 129, 084904.

28. (a) Seifert, U., Phys. Rev. Lett. 2000, 84, 2750-3; (b) Tees, D. F. J.; Woodward, J. T.; Hammer, D. A., J. Chem. Phys. 2001, 114, 7483-96.

29. Rao, J.; Lahiri, J.; Isaacs, L.; Weis, R. M.; Whitesides, G. M., Science 1998, 280, 708-11.

30. Krishnamurthy, V. M.; Estroff, L. A.; Whitesides, G. M., Multivalency in Ligand Design. Wiley-VCH Verlag GmbH \& Co. KGaA: 2006; p 11-53. 
31. The approximate value of koff for a monovalent complex is deduced from the equilibrium constant in solution Keq-M (4.6 $104 \mathrm{M}-1$ ) and a diffusion limited on-rate kon $=108 \mathrm{M}-1 \mathrm{~s}-1$ (see ref. 16a).

32. Ercolani, G., J. Am. Chem. Soc. 2003, 125, 16097-103.

33. Sandal, M.; Benedetti, F.; Brucale, M.; Gomez-Casado, A.; Samori, B., Bioinformatics 2009, 25, 1428-30.

34. RDC-Team, http://www.R-project.org 2008.

35. Sheather, S. J.; Jones, M. C., J. Roy. Stat. Soc. B Met. 1991, 683-90. 



\section{Chapter 6}

\section{Monte Carlo simulations of the spreading of divalent molecules on a receptor surface}

The spreading rate of divalent ligand molecules over receptor-functionalized surfaces has recently been found to have a complex dependence on the competing free receptor concentration, suggesting different mechanisms of spreading are prevailing in different free receptor concentration ranges. A reduced-scale model of the system was developed to study these processes using Monte Carlo simulations. Single- and many-molecule simulations were combined to reproduce our experimentally observed phenomena. Three mechanisms of motion have been considered: (1) partial dissociation (walking), (2) local diffusion of rapidly rebinding species (hopping) and (3) complete dissociation / re-association (flying). Whereas the contribution of walking to the spreading on multivalent surfaces was negligible, the interplay of hopping and flying describes the experimentally observed spreading behavior. The simulations showed a transition from a near-surface mechanism, independent of the simulated solution volume size (hopping) to a bulk mechanism, affected by the height of the solution volume (flying). This change of the preferred spreading mechanism happened at concentrations of free host in solution near $1 \mathrm{mM}$, coinciding with the experimentally observed decrease in spreading rate. 


\subsection{Introduction}

Multivalent interactions play a key role in many biological phenomena, such as immune response, signaling and virus infection. ${ }^{1}$ Achieving a better understanding of multivalent interfacial interactions can extend our knowledge of processes such as cell-cell recognition and endocytosis, as well as enable the development of new materials ${ }^{2}$ and molecular machines. ${ }^{3}$ Although the thermodynamics of multivalent assemblies at interfaces is well studied, their dynamic behavior often remains elusive. In particular, gaining insight in the process of formation of multivalent ligand-receptor complexes at the cell membrane is a challenging task, which requires the careful modeling of aspects such as steric constraints and rate laws. ${ }^{4}$ A synthetic analogue for these systems, substituting the cell-membrane receptor, would consist of $\beta$ cyclodextrin immobilized on gold or silicon dioxide substrates. ${ }^{5}$ In this model system, the role of the ligand is played by dyes, ${ }^{6}$ biological ligands, ${ }^{7}$ dendrimers $^{8}$ or nanoparticles ${ }^{9}$ decorated with one or more guest moieties for $\beta$-cyclodextrin. Very recently, the spreading of multivalent molecules (fluorescently labeled mono-, bis- and tris- adamantyl guests) over a $\beta$ cyclodextrin functionalized surface was reported. ${ }^{10}$ The guests were microcontact printed onto a cyclodextrin-coated glass slide following a line pattern using a polymeric stamp. Then, a drop of aqueous native cyclodextrin solution was deposited on top of this slide. Using fluorescence microscopy we observed the broadening of the printed lines under different concentrations of competing free $\beta$-cyclodextrin in the solution and quantified the spreading rate of the labeled guests over the multivalent surface (Figure 6.1). The rate of spreading was enhanced by the presence of free cyclodextrin in the solution covering the studied surface. However, for concentrations of free host around 1-2 mM the spreading was slowed down in the case of di- and trivalent molecules. These findings suggest that the diffusing guests can follow multiple mechanisms to alter the guest's position. We focused on the divalent case as it seemed the most tractable case due to its relative simplicity. 


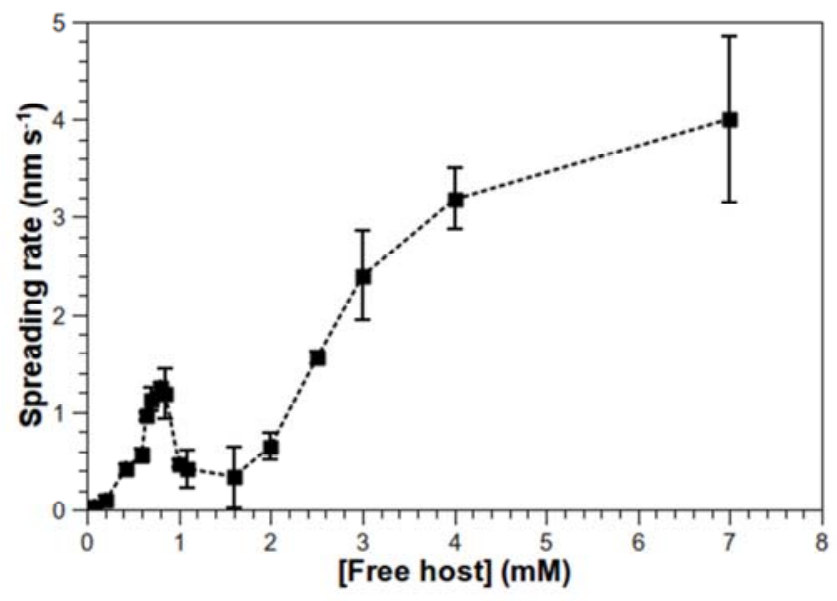

Figure 6.1 Experimental data for the spreading rates of microcontact printed patterns of divalent adamantyl guests over multivalent $\beta$ cyclodextrin-covered surfaces. ${ }^{10}$ Dotted line is a guide to the eye.

Three mechanisms or modes of diffusion are proposed for this multivalent system, (i) complete dissociation and re-association, (nicknamed "flying"), (ii) partial dissociation ("walking") and (iii) local solution diffusion of a partially uncapped, rapidly rebinding species ("hopping"), as depicted in Figure 6.2. In the first mode, "walking" is performed by the divalent guest $(G)$ fully bound to surface $G\left(H_{s}\right)_{2}$ when one of the guest moieties dissociates, forming $G_{s}$, and rebinds to a cavity different than the starting host. The other two modes, "hopping" and "flying", require a complete dissociation of the guest away from the surface. The main difference is that in the case of "hopping" the guest remains locally close to the surface, whereas in the case of "flying" the guest diffuses through the bulk of the solution before rebinding to the surface.

Here the movement of divalent molecules over a receptor surface is investigated by means of Monte Carlo simulations and a reduced-scale model of the system using both many- and single-molecule approaches. The significance of the different spreading mechanisms will be evaluated, leading to a molecular interpretation of the experimental observations. 


\subsection{Methods}

\subsubsection{Statistical model}

To study the spreading mechanisms of the divalent guest over a host-covered surface, a detailed record of the development of the concentration profile of each individual species is needed. To this end, we employed Gillespie's algorithm, ${ }^{12}$ a variety of dynamic Monte Carlo, which has been used to simulate chemical and biochemical systems of reactions. ${ }^{13}$ The original Gillespie method applies to well-mixed systems, and hence is unable to describe spatial inhomogeneity. More recently an extended method including pseudo-reactions to simulate diffusion processes has been developed. ${ }^{14}$ We adopted this extended Gillespie algorithm to follow the positions and populations of the guests in their respective state of complexation. The algorithm was implemented in $\mathrm{C}$ language and the simulations were performed with an Intel Pentium 4 desktop computer. The fact that each iteration of the algorithm is a single reaction imposes a severe limitation on the number of molecules that can be simulated in an acceptable real-time period, and thus on the size of the simulated system. Therefore we decided to model the multivalent surface as a three-host-wide line with lateral periodic boundaries to avoid border artifacts, as depicted in Figure 6.3. Volumes of the solution on top of each triad of hosts, with a height of one host diameter, are considered as sub-volumes.

The characteristic reaction time for the fastest interaction step involving solution species is larger than the average time a molecule spends in one of these sub-volumes, hence the contents of each sub-volume can be considered well-mixed. Diffusion of the species between each sub-volume can be included in the algorithm as a reaction, with its rate constant deduced from the diffusion coefficient and the characteristic length of the sub-volume. The diffusion constant is assumed to be the same for every species in solution, considering that their masses are of the same order of magnitude. We take into account that singly bound divalent guests $\left(\mathrm{GH}_{\mathrm{S}}\right)$ can only access their immediate neighboring cavities, considering the length of the chain connecting the two guest moieties. 


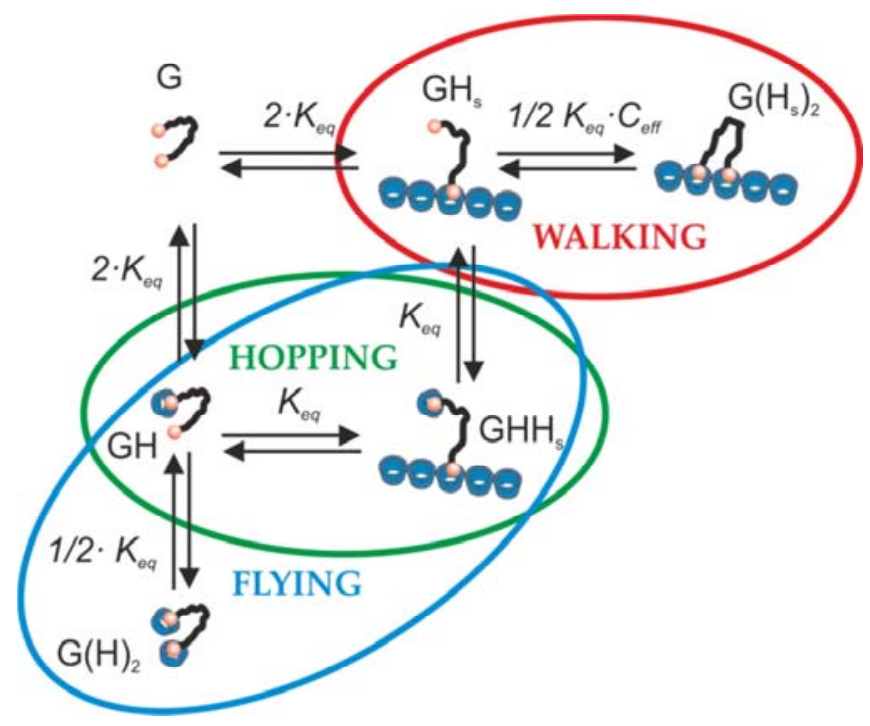

Figure 6.2 Scheme of the possible reactions ${ }^{11}$ on the modeled system. The three potential mechanisms of spreading are identified. Species are named after their components $G$ (guest), $H$ (free host) and $H_{s}$ (surfacebound host).

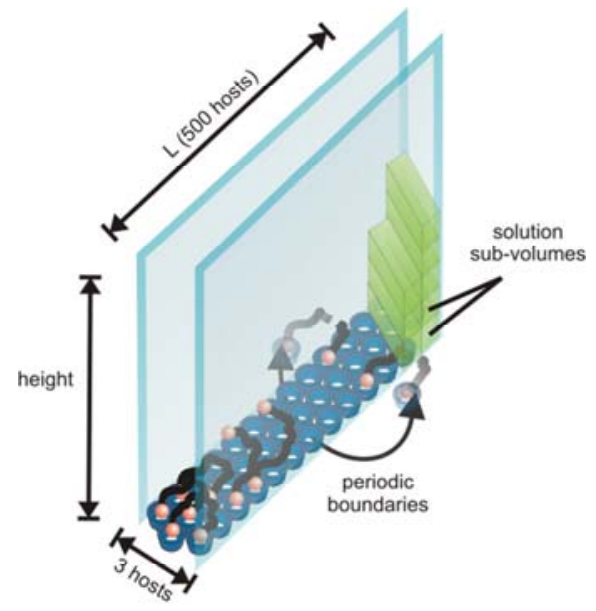

Figure 6.3 Representation of the simulated system. The host-coated surface (molecular printboard) is modeled as a hexagonally packed (approximately $2 \mathrm{~nm}$ lattice constant) three-host wide line with circular boundary conditions. The solution volume is divided in sub-volumes of $3 \times 1 \times 1$ host cavities $\left(6 \times 2 \times 2 \mathrm{~nm}^{3}\right)$. 
After each surface reaction the host surface is examined and an effective concentration for each $\mathrm{GH}_{\mathrm{S}}$ is calculated based on the availability of its surrounding six cavities and the volume of the semi sphere defined by the maximum distance between the two guest moieties. ${ }^{15}$ Furthermore, guests which reside in the sub-volumes immediately on top of the surface are treated by assigning a probability of rebinding dependent on the local availability of free surface-bound hosts. The number of free hosts in solution is typically higher than any other species and therefore this species can be treated as homogeneously distributed in the solution (reducing the simulation times by orders of magnitude).

\subsubsection{Simulation parameters}

The values of the rate and diffusion constants used in this simulation (Table 6.1) have been reported in previous studies ${ }^{15-16}$ or where deduced from them. The sizes of the simulated host surface and solution volume were chosen to yield reliable statistics within acceptable simulation times. The number of free host molecules in solution was adjusted to match the concentrations used in the experiments, ranging from 0 to $1.5 \mathrm{mM}$. For the full system simulations, 300 cavities on one side of the simulated stripe were randomly initialized by occupying them with fully bound divalent guests (about 140 molecules), keeping the total host occupation around 95\%, in agreement with experimental findings. ${ }^{15,17}$ The dependence of the spreading behavior on the height of the solution volume was examined in the simulations. In the case of the single molecule simulations one divalent guest was placed on the center of the threehost-wide stripe and the reaction rates were adjusted to prevent divalent attachment, in order to artificially enhance the occurrence of the hop and fly modes of movement. For these simulations the height of the volume was kept the same as in the full system simulations.

\subsubsection{Output analysis}

The basic output of the simulation is a series of text files containing the occupation of the surface and solution at given times, as well as the population and reaction counts for each species. The latter can be examined and plotted directly with spreadsheet applications. Visualization of the snapshots is not so 
straightforward, since they include several thousands of files with numerical data. A Python ${ }^{18}$ script was used to average the surface state of several simulations, as well as to stack these states according to the time they were taken. This new numerical file can be used for further analysis or opened with other applications (here Image ${ }^{19}$ ) to visualize the time evolution in a graphical manner. The time evolution of the occupation of the surface-bound host was fitted to a one-dimensional diffusion model. We defined the point that leaves half of the bound population to either side as "midpoint" and used the output to obtain pairs of (time, midpoint). The time evolution of this particular point can be deduced from the analytical diffusion equations, and thus obtaining an apparent diffusion constant from a fit of these pairs (See Appendix A).

\subsection{Results and discussion}

\subsubsection{Full system simulations}

In an attempt to shed light upon the origin of the decay in spreading rate in the 1-2 $\mathrm{mM}$ range of competing free host, every possible reaction was modeled as given in Figure 6.2. Since the equilibrium populations were not able to account for the observed spreading rate decay in the 1-2 $\mathrm{mM}$ range of concentration of free host, ${ }^{10}$ Gillespie's algorithm was used to capture the kinetic behavior of the system. To assign values to the reaction rates and diffusion constants in the model previously reported data (Table 6.1) were used. We consider that the $\mathrm{G}(\mathrm{H})_{2}$ species is not involved in the "hopping" process for the reason that the complete capping with free host allows it to diffuse for relatively long times and far from the surface before any of the two guest moieties dissociates (See Appendix B). Although the species named $G$ is not included in any of the modes in Figure 6.2, since its presence and lifetime in solution is extremely short, the statistical model incorporates it for all the calculations and its contribution to spreading is accounted for. 


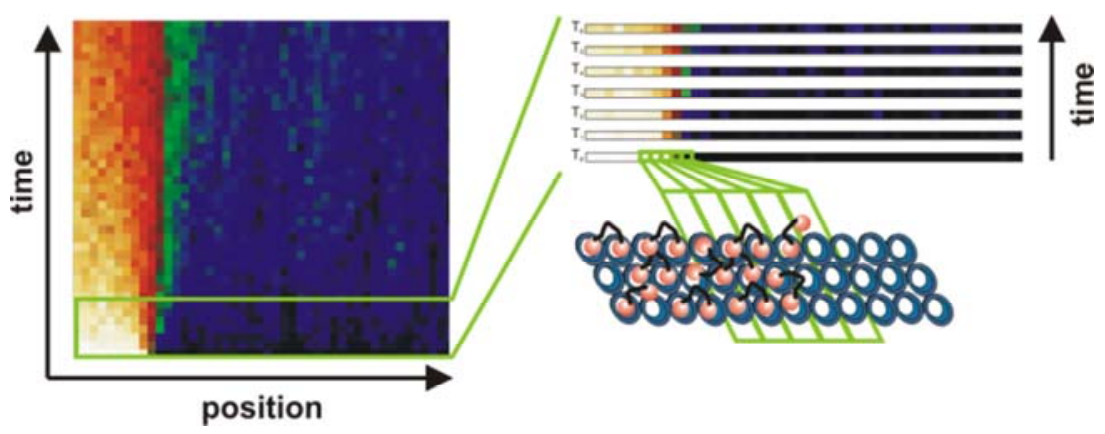

Figure 6.4 Construction of a time evolution map: each three-host-wide section is assigned to an intensity value according to its occupation, and the obtained lines are stacked vertically.

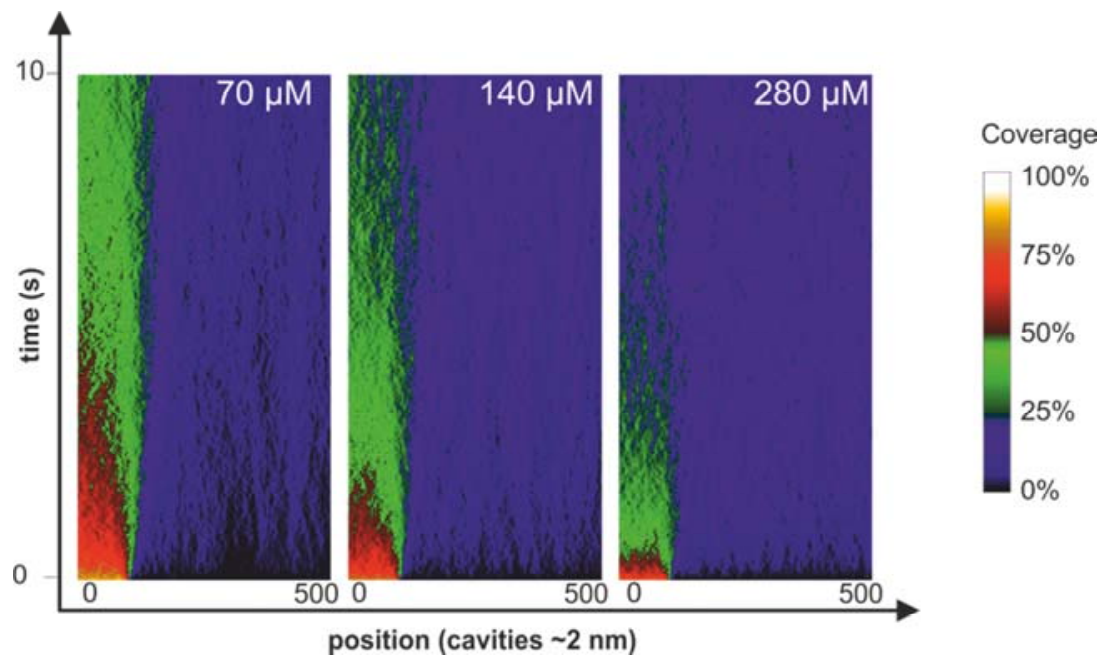

Figure 6.5 Averaged time evolution maps (from ten runs) of the system for different concentrations of free host in solution. The vertical direction denotes the simulation time $(0-10 \mathrm{~s})$ and the horizontal direction positions the multivalent host surface. Each line of the images represents a snapshot taken every $0.01 \mathrm{~s}$. 


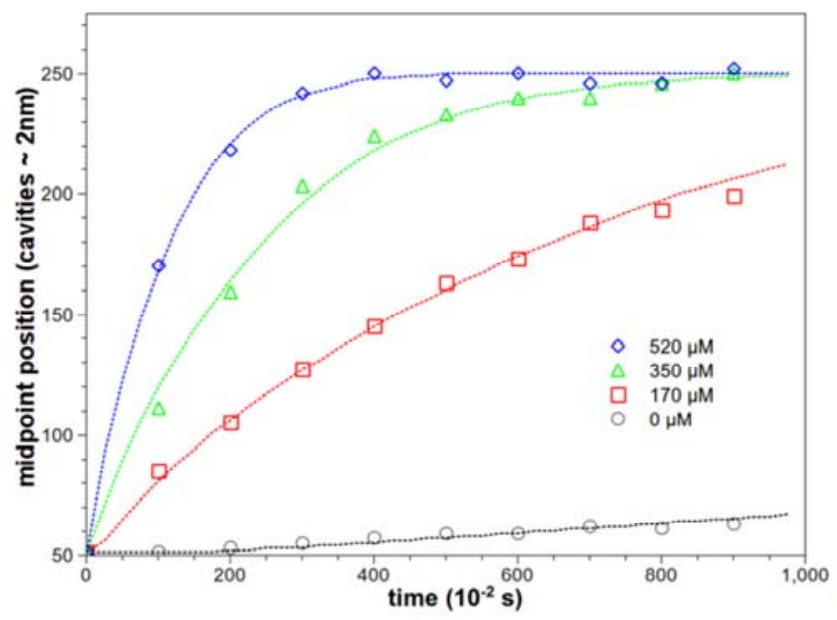

Figure 6.6 Time evolution of the center point of the surface-bound guest population derived from the simulations (symbols). The lines are fits to the data with a one-dimensional diffusion for different concentrations of free host in solution.

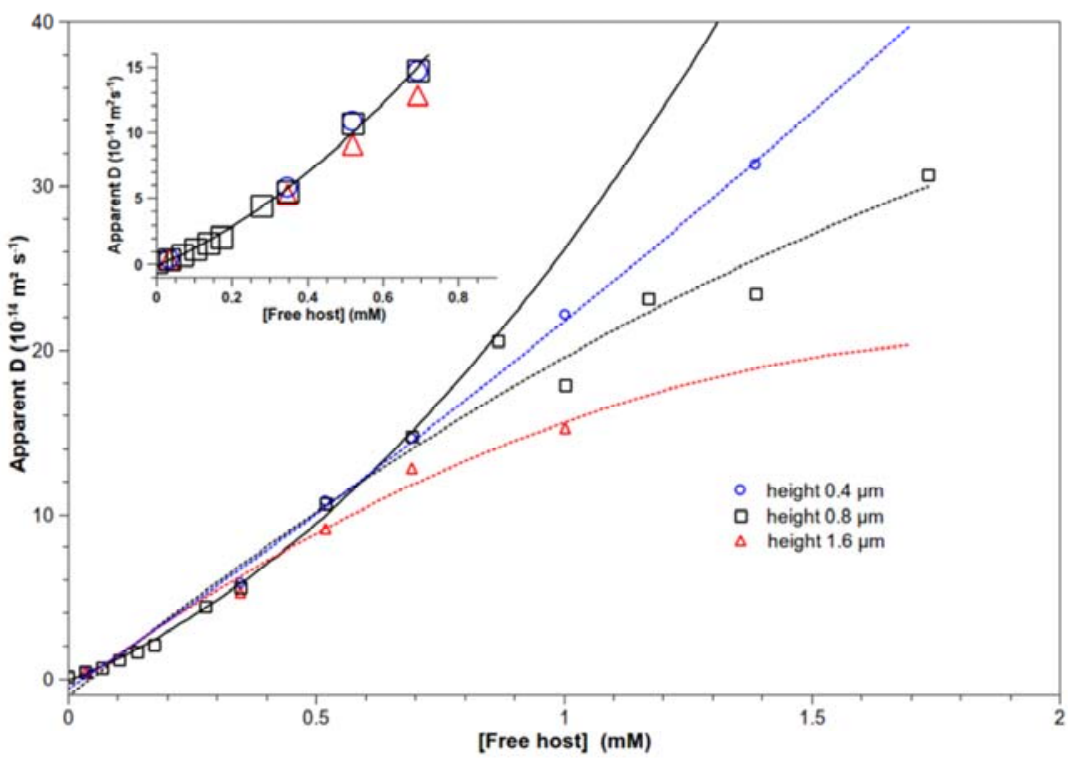

Figure 6.7 Apparent diffusion constant of the bound guest versus free host concentration for different solution volumes as determined by fitting midpoint time evolution graphs as shown in Figure 6 . The lines are guides to the eye, (solid: second order fit of the volume independent regime, dotted: second order fit of data from smaller to larger height). Inset: magnification of the low host concentration range. 
Since Gillespie's method selects and executes reactions one by one for all the species present in the system, only a low number of molecules and reactions can be followed. It also assumes that the species are well mixed in solution, which is not the case in our experimental situation. Therefore the system was scaled down to restrict simulation times (up to ten days for the highest concentration of competing host in solution, $1.7 \mathrm{mM}$ ), and the algorithm was extended to deal with local concentrations and reactions involving surface hosts. Simulations involving around 140 guest molecules, all divalently bound (corresponding to ca. 95\% occupation of the leftmost 3×100 cavities of the host surface), were performed while changing the total amount of free host in the top solution volume. The output of these simulations was visualized by assigning a color value to the state of each three-host section of the surface, as shown in Figure 6.4. After stacking these colored lines, a time evolution picture was constructed. Ten simulation runs were averaged for each condition to smoothen the time evolutions.

Table 6.1 constants and parameters used for the simulations.

\begin{tabular}{|c|c|c|}
\hline \multicolumn{3}{|c|}{ System geometry } \\
\hline $\mathrm{L}$ & Length of stripe & $1 \mu \mathrm{m}$ (500 cavities) \\
\hline W & Width of stripe & $6 \mathrm{~nm}$ (3 cavities) \\
\hline $\mathrm{H}$ & Solution volume height ${ }^{\mathrm{a}}$ & $0.4 / 0.8 / 1.6 / 3.2 \mu \mathrm{m}$ \\
\hline & Solution sub-volume dimensions ${ }^{b}$ & $2 \times 2 \times 6 \mathrm{~nm}^{3}$ ( $1 \times 1 \times 3$ cavities $)$ \\
\hline \multicolumn{3}{|c|}{ Reaction constants } \\
\hline $\mathrm{K}_{\mathrm{eq}}$ & Intrinsic host-guest equilibrium constant ${ }^{\mathrm{c}}$ & $5 \cdot 10^{4} \mathrm{M}^{-1}$ \\
\hline $\mathrm{k}_{\mathrm{a}}$ & Association rate constant $^{d}$ & $10^{8} \mathrm{M}^{-1} \mathrm{~s}^{-1}$ \\
\hline $\mathrm{k}_{\mathrm{d}}$ & Dissociation rate constant $^{d}$ & $2 \cdot 10^{3} s^{-1}$ \\
\hline \multicolumn{3}{|c|}{ Other parameters } \\
\hline $\mathrm{C}_{\text {eff }}$ & Effective concentration $^{\mathrm{e}}$ & $0.2 \mathrm{M}$ \\
\hline $\mathrm{D}$ & Diffusion constant in solution $^{f}$ & $10^{-10} \mathrm{~m}^{2} \mathrm{~s}^{-1}$ \\
\hline$[\mathrm{H}]$ & Free host concentration $^{g}$ & $0-1.7 \mathrm{mM}$ \\
\hline
\end{tabular}

a) The value $0.8 \mu \mathrm{m}$ was chosen for most part of the study, the value $3.2 \mu \mathrm{m}$ was only used in single-molecule simulations. b) Ensures many more diffusion reactions than (un)binding reactions, necessary for the well-mixed approximation. ${ }^{14} \mathrm{c}$ ) Determined by calorimetry. ${ }^{15} \mathrm{~d}$ ) See Chapter 5. ${ }^{15,20}$ e) As reported. ${ }^{15} \mathrm{f}$ ) Same order of D value $3 \cdot 10^{-10} \mathrm{~m}^{2} \mathrm{~s}^{-1}$ for $\beta$-cyclodextrin. ${ }^{16} \mathrm{~g}$ ) The range was extended up to $2.7 \mathrm{mM}$ for single-molecule simulations. 
The averaged time evolution maps (Figure 6.5) confirm the enhanced spreading rate of the divalent guest upon increasing concentration of free host in the solution volume on top of the surface. To quantify the spreading, the "midpoint" of the bound guest, i.e. the point of the host surface that contained the same amounts of bound guest on either side at a given time, was calculated.

The evolution of this "midpoint" in time was fitted to a one-dimensional diffusion model to yield an apparent diffusion constant for each concentration of host (See Appendix A). As can be seen in Figure 6.6, the simulated spreading is well described as a one-dimensional diffusion process. An apparent diffusion constant was determined in this way for each of the free host concentrations in a solution volume with a height of $0.8 \mu \mathrm{m}$.

In Figure 6.7 the apparent diffusion constant is plotted against the free host concentration (squares). For concentrations below $1 \mathrm{mM}$ the effective diffusion coefficient increases with concentration according to a second order polynomial fit (solid line), but starts to deviate from this trend as the concentration increases further. Since the experimentally observed spreading rate and the simulated apparent diffusion constant are related, we expected that the empirically determined decay in spreading rate around 1-2 $\mathrm{mM}$ of free host (Figure 6.1) should be represented by a similar trend in the simulated apparent diffusion constant. Although in this simulation no decay was observed, the change occurs in the same range of concentrations where the decay in the real experiments started, hinting at a possible change of spreading mode. The simulations were repeated for several of the free host concentrations showing that for concentrations over $1 \mathrm{mM}$ the spread of the data was increasing, which can be explained by the random differences in the number and elapsed time of "flying" processes occurring in these conditions.

The influence of the solution volume was investigated at different free host concentrations by changing the height of the compartment from one half $(0.4$ $\mu \mathrm{m})$ of the initially selected value $(0.8 \mu \mathrm{m})$ to two times this value $(1.6 \mu \mathrm{m})$. As shown in Figure 6.7 (dots and triangles), the apparent diffusion constant was independent of the volume size for concentrations of free host in solution below $0.8 \mathrm{mM}$, whereas in the case of concentrations above this value a dependence was observed. The deviation from the second order trend is more pronounced for larger volumes, showing that the moderate trend change for smaller heights is an artifact produced by the limited volume size. More 
importantly, a transition occurs from a volume independent (local) mode of spreading below $0.8 \mathrm{mM}$ to a volume dependent (in-bulk) mode above $0.8 \mathrm{mM}$, which correspond to the characteristics of the proposed modes "hopping" (local) and "flying" (in-bulk). This transition is happening in the concentration range where the decay of spreading rate was observed experimentally. We expect that simulating even larger volumes with concentrations of free host ranging 1-2 mM would show a more pronounced decay similar to the experimental findings, however due to long run times for these conditions (estimated on the order of months per condition set) and increasing noise this is unpractical.

\subsubsection{Single molecule simulations}

Considering the observation that spreading is very slow when free host is absent, under which conditions "walking" is more effective, ${ }^{10}$ this mode can be neglected with respect to "hopping" and "flying" modes in the presence of free host in solution. Moreover, addition of free host binds partially uncomplexed guests $\mathrm{GH}_{\mathrm{s}}$ to give $\mathrm{GHH}_{\mathrm{s}}$, inhibiting this walking process for increasing free host concentration. Therefore, only "hop" and "fly" modes should be considered to play an important role in the spreading phenomena for this system. To test the relative importance of the "hop" and "fly" mechanisms, reaction parameters were modified in order to simulate a single divalent guest that can only bind monovalently to the host surface (forming $\mathrm{GH}_{\mathrm{s}}$ or $\mathrm{GHH}_{\mathrm{s}}$ was allowed, but the formation of $\mathrm{G}\left(\mathrm{H}_{\mathrm{s}}\right)_{2}$ was forbidden), but is still able to form a complex with two hosts if at least one of them is a free host in solution (i.e. divalent complexes $\mathrm{GHH}_{\mathrm{s}}$ and $\mathrm{G}(\mathrm{H})_{2}$ were both allowed). In this way, this hypothetical guest was forced to perform only "hop" or "fly" movements and resulted in an increased number of these movements, but without altering their relative ratio. Since only one guest molecule is being tracked, both the size of the solution volume and the host concentration range can be simulated for values that were unpractical in the full system situation. Time evolution maps were constructed by taking snapshots only when the single guest was bound to the surface (Figure 6.8). For low free host concentrations the molecular movement was restricted by far locally, while at higher concentrations of free host in the top volume a gradual increase of the amount of non-local displacements was observed in a more fragmented trajectory. By averaging ten runs of simulations 
reliable statistics for the amounts and lengths of the guest movements in these conditions were achieved.

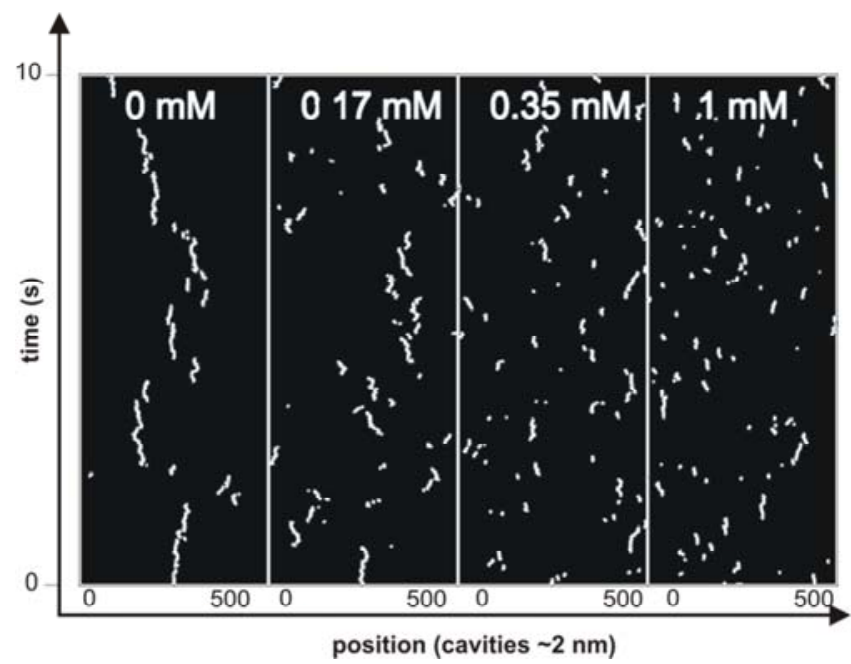

Figure 6.8 Time evolution for a single divalent guest that can only bind monovalently to the surface. The individual white pixels have been enlarged for easier view.
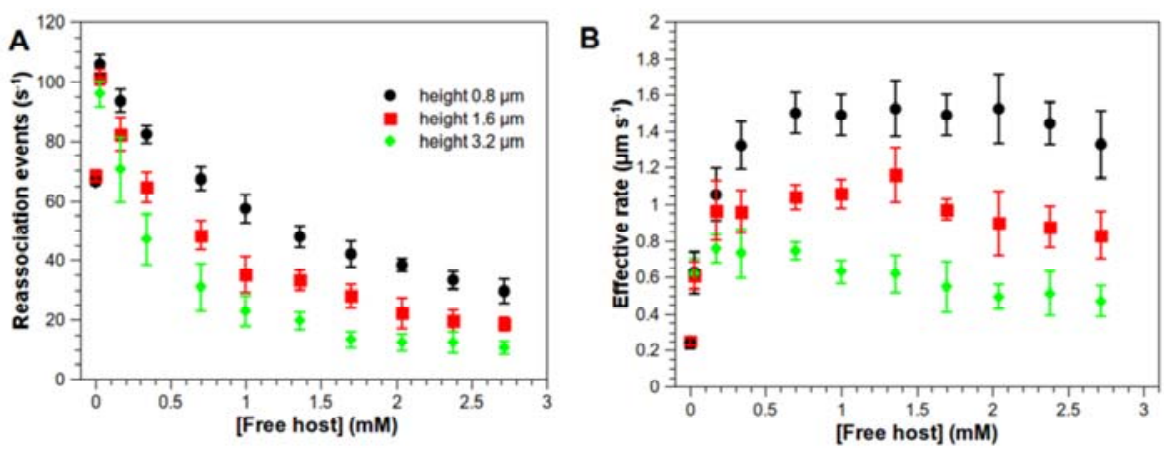

Figure 6.9 Results from single molecule simulations. A) Number of reassociation events to the surface. B) Effective rate. Solution volumes correspond to the full system conditions, with a height of $0.8 \mu \mathrm{m}$ (dots), $1.6 \mu \mathrm{m}$ (squares) and $3.2 \mu \mathrm{m}$ (diamonds). 
Moreover, the effect of increasing the size of the solution volume was investigated. Figure 6.9 represents the collected statistics on the amount and characteristics of these movements. The number of reassociation events to the surface (considering only unbind-diffuse-rebind processes, i.e processes where no diffusion takes place between dissociation from a host and association of the guest to that same surface host were excluded) decreased with increasing host concentration in solution as well as with the volume size (Figure 6.9A). In addition, an effective rate was calculated by measuring the distance between two consecutive binding positions and the time necessary for the guest to travel such a distance. The graph in Figure 6.9B indicates that at a free host concentration of $0 \mathrm{mM}$ the effective rate is very low. Under these conditions the guest is subject to short-range hops, which is plausible since there is no competing host to cap any of the two guest moieties and the lifetime of such an uncapped divalent guest in solution is short because of the presence of many surface receptors sites in close vicinity. Addition of competing host leads to fragmentation of the trajectory of the guest on the surface, indicating that movements are covering larger distances. This can be attributed to two situations, the earlier mentioned "hop" and "fly" modes. The increase of binding events when free host is present suggests that the guest can now be partially capped by a free host, thereby increasing its lifetime in solution and thus allowing it to stay unbound long enough to reach neighboring cavities, performing a so-called "hop" movement instead of rebinding to the starting host. Further increase of the host concentration in solution enhances the probability that the guest will be doubly capped by a competing host, augmenting the probability of traveling into the bulk of solution, performing a so-called "fly" movement, and thus promotes "flying" instead of "hopping". Although "fly" lengths can be much longer than "hop" lengths, the time that the molecule spends trapped in the bulk of solution paces down the effective speed of this "flying" mode (See Appendix B).

Subsequently, the simulations were repeated for larger heights. The number of times that the molecule binds back to the surface decreased with the solution height, and the effective rate showed a maximum followed by a decay upon increasing the free host concentration, while the speed was lower for larger volumes (Figure 6.9). Again, these observations are in agreement with our interpretation of a shift from spreading by "hopping" to spreading by "flying", which makes the effective diffusion over the surface slower. When having a small solution height this decay of effective spreading rate can be prevented. If 
in this case one of the bound native hosts dissociates (forming $\mathrm{GH}$ ), guest molecules of the $\mathrm{G}(\mathrm{H})_{2}$ species will remain close enough to the surface to readily bind back to the surface and form $\mathrm{GHH}_{\mathrm{s}}$.

\subsubsection{Molecular picture of the spreading mechanisms}

The results from both many- and single-molecule simulations suggest that the "hopping" mechanism is responsible for the complex spreading behavior, being activated by the presence of free host concentration but inhibited when this concentration reaches a certain value that makes flying processes more likely, as depicted in Figure 6.10.

We expect that many-molecules simulations would present a clearer transition using larger solution volumes and numbers of guests, which are limited by simulation run times. On the other hand, the single-molecule simulations allowed us to overcome these limitations, since their run times were much shorter and there was only one molecule to keep track of, at the cost of losing information on the changes of the species population with free host concentration. Therefore the information collected from the two approaches was combined focusing on the "hopping" mode, as can be seen in Figure 6.11. We reasoned that the spreading by "hopping" must be directly proportional to three factors. The first factor is the population of the $\mathrm{GHH}_{\mathrm{s}}$ (surface-bound guests capped with one host from solution) species (Figure 6.11A), which was obtained from the many-molecules simulation (in agreement with the predictions from the thermodynamic equilibrium equations for this system). ${ }^{10}$ Additional factors are: the chance of performing a "hop" instead of a "fly" (Figure 6.11B), and the average distance covered on one of these "hops" (Figure 6.11C), both of which can be extracted from the single-molecule simulations. The "hop" distance $d_{\text {hop }}$ is also in agreement with the estimated free diffusion length of a singly-capped guest (dotted line in Figure 6.11C) before encountering a second host from solution, in which case the movement would be a "fly" (See Appendix C). When these three factors were multiplied the thus defined rate shows a local maximum for a concentration of free host around $1.5 \mathrm{mM}$ (Figure 6.11D). Although the experimentally observed maximum was at lower concentration $(0.8 \mathrm{mM})$ and the decrease in spreading rate was more pronounced, the resemblance seems reasonable considering the many approximations and limitations of our approach. 


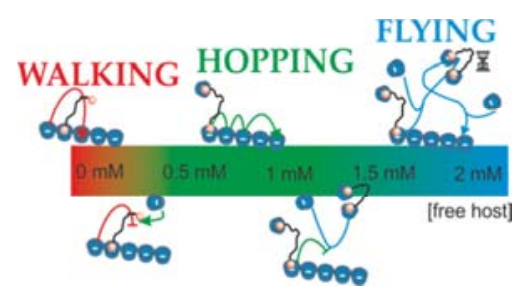

Figure 6.10 Proposed mechanism for surface diffusion and the dependence on concentration of free host in solution. The slow mode of walking is inhibited by the presence of free host, which creates increasing populations of monovalently capped guest $\mathrm{GHH}_{s}$, which can move by hopping. Further addition of free host to the solution increases the chance of doubly capping the hopping guest $\mathrm{GH} \rightarrow \mathrm{G}(\mathrm{H})_{2}$, inhibiting this mode and favoring movement by flying.
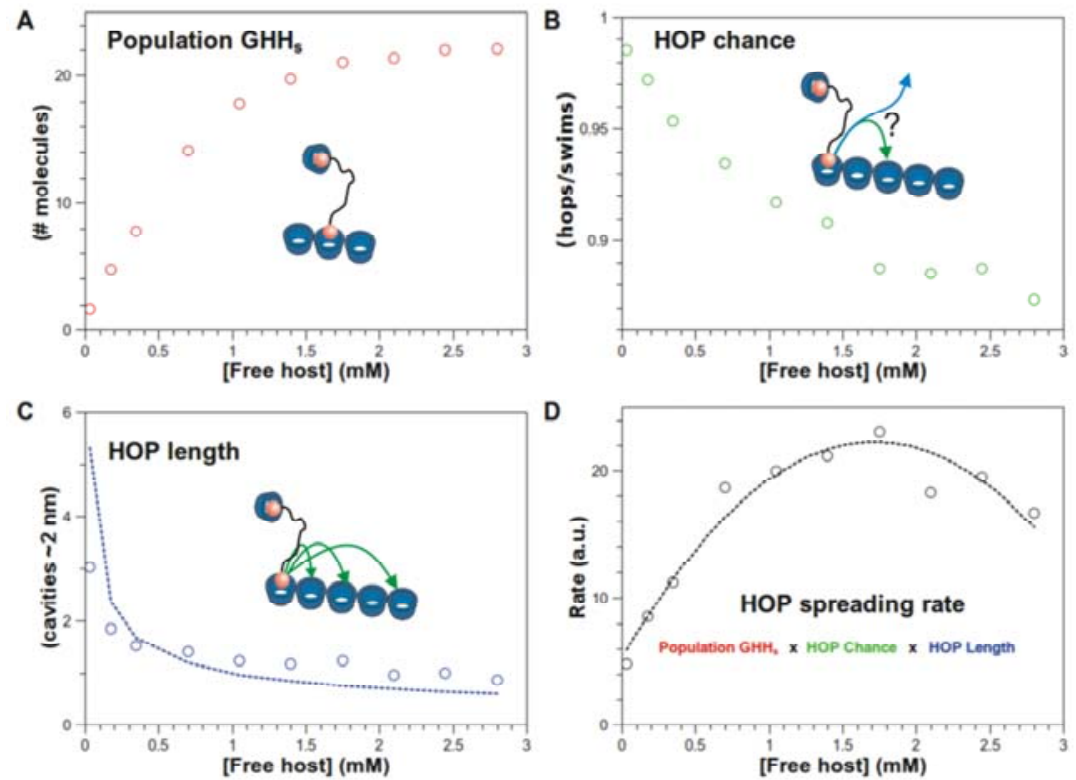

Figure 6.11 Estimation of the spreading rate originating from the hopping mode. A) Population of monovalently capped guest $\mathrm{GHH}_{\mathrm{S}}$ derived from full system simulations; B) chance of performing a movement by hopping derived from single molecule simulations; C) length of movement by hopping derived from single-molecule simulations and predicted diffusion length (line, see Appendix C); D) combination of the three factors and second order fit as a guide to the eye (line). 


\subsection{Conclusions}

A microscopic explanation for the complex spreading behavior of divalent guests on host-covered surfaces, previously experimentally observed, is presented in this study. Three possible modes of spreading, "walking", "hopping" and "flying" have been identified, which may be followed by a divalent guest to alter its position over a host surface. One of them, "walking", appears to have a negligible contribution to the total spreading, as can be confirmed by the very slow changes in both experiments and simulations where no free host was present in the system. In these conditions "hopping" and "flying" are also scarce, since the only possible free guest species (G) has a very low population and lifetime in solution. Addition of free host decreases the population of the species involved in "walking", reducing even further the significance of this mode. Therefore, the major part of guest transport must occur through "hopping" and "flying" modes. Using many- and single-molecule Monte Carlo simulations this spreading phenomenon was dissected into different regimes of free host concentration where the transport is more or less affected by the height of the closed volume on top of the studied surface. Associating volume-independent transport to the "hopping" mode and volumedependent transport to the "flying" mode offers an explanation for the experimentally observed decrease in spreading rate. A transition from a merely "hopping" mode, in the regime of low free host concentration (under $1 \mathrm{mM}$ ), to the less effective "flying" mode (higher than $1 \mathrm{mM}$ ) accounts for the experimental results.

\subsection{Appendix A: Mathematical derivations for diffusion constant fitting}

The time evolution maps derived from the full system simulations (Figure 6.5) were analyzed to quantify the spreading observed in them. The diffusion on the host-covered surface was approximated as a case of Fick's one-dimensional diffusion.

First, a general expression for the concentration at any time was deduced. Let $C(x, t)$ be the distribution of guest bound to the surface. The simulation conditions are matched by setting the initial state as an homogeneous distribution over one fifth of the surface and nothing over the rest (Figure 
6.12). In the experiment the guest was printed in evenly spaced lines, so we can assume $C(x, t)$ is periodic and symmetric, thus we only need to study it in one of the periods.

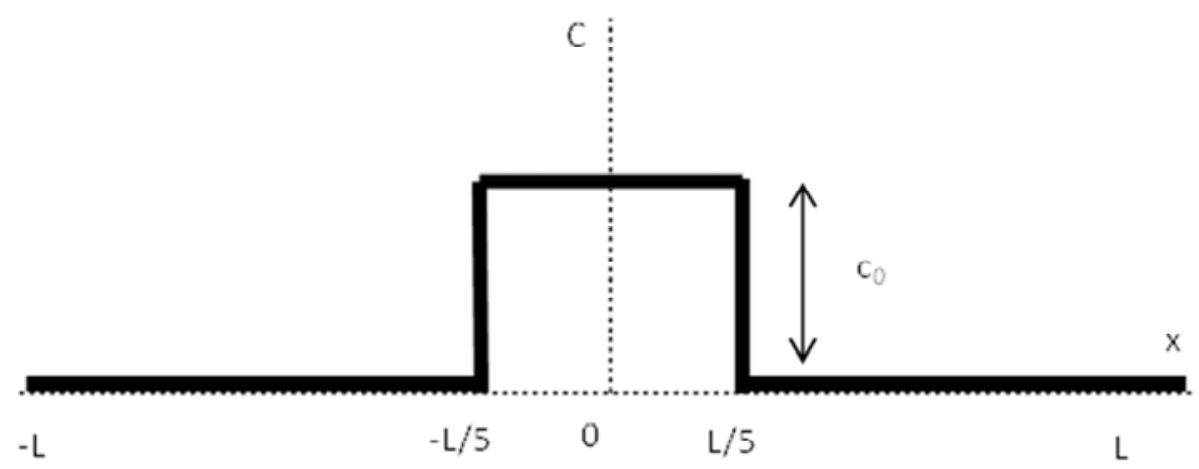

Figure 6.12 Scheme of the initial state $C(x, t=0)$.

The evolution of the bound guest is modeled as a 1D diffusive process, which is described with the full value problem:

$P D E: \frac{\partial C(x, t)}{\partial t}=D \frac{\partial^{2} C(x, t)}{\partial x^{2}}, \quad-L<x<L, \quad t>0$

with the boundary conditions:

$B C_{1}: \frac{\partial C(L, t)}{\partial x}=0$

$B C_{2}: \frac{\partial C(-L, t)}{\partial x}=0$

and the initial conditions:

IC: $C(x, 0)=\left\{\begin{array}{lr}C_{0} & \text { for }-\mathrm{L} / 5<\mathrm{x}<\mathrm{L} / 5 \\ 0 & \text { otherwise }\end{array}\right.$

Separation

$C(x, t)=X(x) T(t)$

gives the Sturm-Liouville (SL) problem for $X(x)$

$O D E: X^{\prime \prime}(x)=K X(x)$ 
$B C_{1}: X^{\prime}(L)=0$

$B C_{2}: X^{\prime}(-L)=0$

with the associated ODE for $\mathrm{T}(\mathrm{t})$

$T^{\prime}(t)=D K T(t)$

The SL-problem has eigenvalues and eigenfunctions

$K \_n=-\lambda \_n^{2}$

$X_{n}(x)=\cos \left(\lambda_{n} x\right), \quad \lambda_{n}=\frac{n \pi}{2 \mathrm{~L}}, \quad n=0,2,4 \ldots$

$X_{n}(x)=\sin \left(\lambda_{n} x\right), \quad \lambda_{n}=\frac{n \pi}{2 \mathrm{~L}}, \quad n=1,3,5 \ldots$

with the associated $T(t)$

$T_{n}(t)=\exp \left(-\lambda_{n}^{2} \mathrm{Dt}\right)$

The Fourier coefficients are found as

$A_{0}=\frac{1}{2 \mathrm{~L}} \int_{-L / 5}^{L / 5} c_{0} d x=\frac{c_{0}}{5}$

$A_{n}=\frac{1}{L} \int_{-L / 5}^{L / 5} c_{0} \sin \left(\frac{n \pi x}{2 L}\right) d x=0$ for $n=1,3,5 \ldots$

$A_{n}=\frac{1}{L} \int_{-L / 5}^{L / 5} c_{0} \cos \left(\frac{n \pi x}{2 L}\right) d x=0 \quad$ for $n=2,4,6 \ldots$

The final solution for the concentration at any point and time will be

$C(x, t)=\frac{c_{0}}{5}+\sum_{n=1}^{\infty} \frac{2 c_{0}}{n \pi} \sin \left(\frac{n \pi}{5}\right) e^{-\left(\frac{n \pi}{L}\right)^{2} D t} \cos \left(\frac{n \pi x}{L}\right)$

where we have renumbered the series as the odd terms drop out because their Fourier coefficients are zero. 
It would be possible to fit this series (truncated to any desired precision) to the intensity profiles of sections of the evolution maps (Figure 6.5) taken at selected times. However, a more convenient procedure is fitting the position of the center of mass (defined as "midpoint" $p$, Figure 6.13) of the bound guest respect to time.

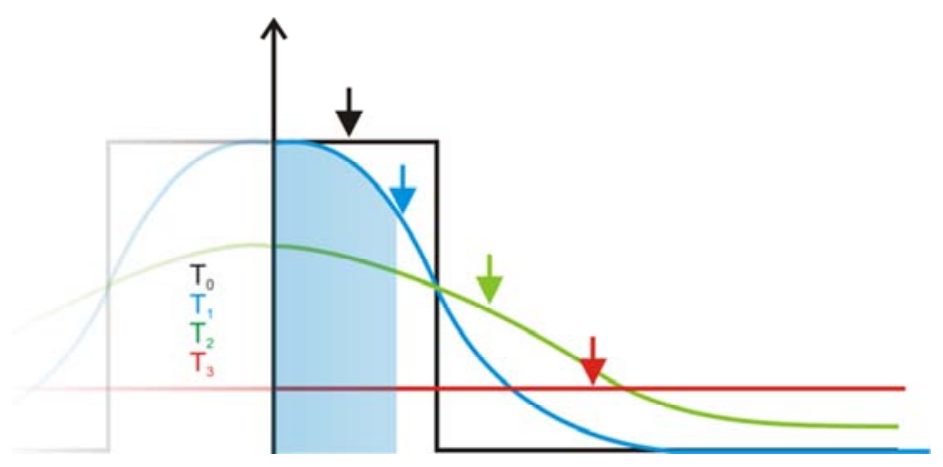

Figure 6.13 Scheme depicting the position of midpoint (arrows) at different times. Midpoint is such that the areas under $C\left(0<x<p_{i}, T_{i}\right)=$ $C\left(p_{i}<x<L, T_{i}\right)$.

Following this definition midpoint must fulfill the following equality:

$$
\int_{0}^{p} C(x, t) d x \stackrel{\text { def }}{=} \frac{1}{2} \int_{0}^{L} C(x, t) d x=\frac{c_{0} L}{10}
$$

Substituting the previously obtained series expansion of $C(x, t)$ we can get

$$
\frac{p}{5}+\sum_{n=1}^{\infty} \frac{2 L}{(n \pi)^{2}} \sin \left(\frac{n \pi}{5}\right) \sin \left(\frac{n \pi p}{L}\right) e^{-\left(\frac{n \pi}{L}\right)^{2} D t}=\frac{L}{10}
$$

This final expression for $p$ can be solved numerically for any $t$ and $D$. As we can extract pairs of $p, t$ from the simulations, we can use these to fit the diffusion coefficient $D$ and quantify the spreading process (Figure 6.6 and Figure 6.7). 


\subsection{Appendix B: Estimation of spreading through the bulk of solution (flying)}

Some simple calculations were performed to estimate characteristics of the flying mode, such as the expected lifetime, traveled distance and speed of a $\mathrm{G}(\mathrm{H})_{2}$ complex (refer to Figure 6.2 for the naming of the different species). In all of these calculations, the values for the reaction rate constants $k_{a}$ and $k_{d}$ and diffusion constant $D$ are taken from Table 6.1.

A $G(H)_{2}$ complex in solution has a chance to rebind to the host-covered surface after dissociating from one of the capping host when it is in the near vicinity of the surface. We can estimate the average time $\tau$ a recently dissociated $\left(\mathrm{G}(\mathrm{H})_{2}\right.$ $\rightarrow \mathrm{GH}+\mathrm{H}$ (free) ) spends in solution without being recapped ( $\mathrm{H}$ (free) $+\mathrm{GH} \rightarrow$ $\mathrm{G}(\mathrm{H})_{2}$ ), and the distance $d_{G H}$ it can travel during that time.

$$
\begin{gathered}
\tau \approx \frac{1}{k_{a}[H]} \\
d_{G H} \approx \sqrt{4 \mathrm{D} \tau}
\end{gathered}
$$

If the solution volume has a height $h$, the probability of the $\mathrm{G}(\mathrm{H})_{2}$ complex dissociating close enough to rebind to the host surface will be approximately:

$P_{\text {rebind }}([H], h)=\frac{d_{G H}}{h}=\frac{\sqrt{4 \mathrm{D} /\left(k_{a}[H]\right)}}{h}$

Using this estimated probability the residence time $T_{1 / 2}$ (average time the complex will spend in solution) can be calculated. We find how many times $n$ the complex should dissociate until the probability of rebinding $P_{\text {rebind }}$ is higher than the probability of remaining free $P_{\text {free }}$ in solution.

$P_{\text {free }}([H], h, n)=\left(1-P_{\text {rebind }}\right)^{n}$

where $n$ is the smallest natural number that makes $P_{\text {free }}<P_{\text {rebind }}$.

$P_{\text {free }}([H], h, n)<\frac{1}{2}$

and the associated residence time, scaled by the rate of dissociation:

$T_{1 / 2}=\frac{n}{2 k_{d}}$ 


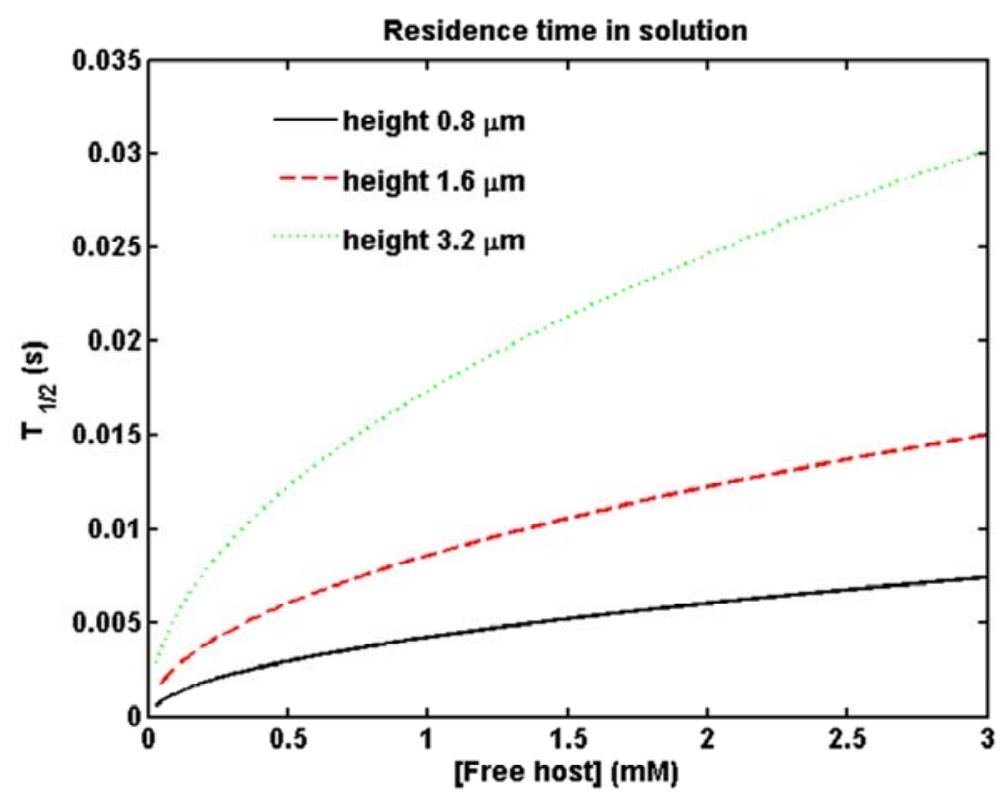

Figure 6.14 Residence time in solution versus free host concentration for a flying guest.

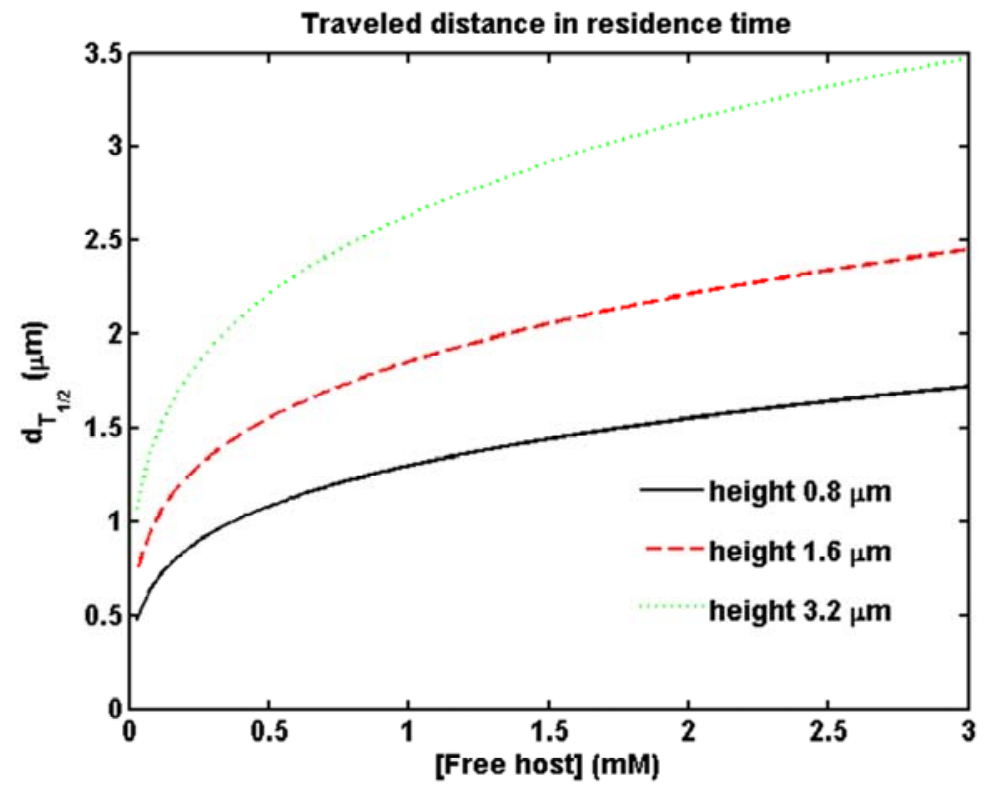

Figure 6.15 Estimated travel distance during the residence time versus free host concentration for a flying guest. 


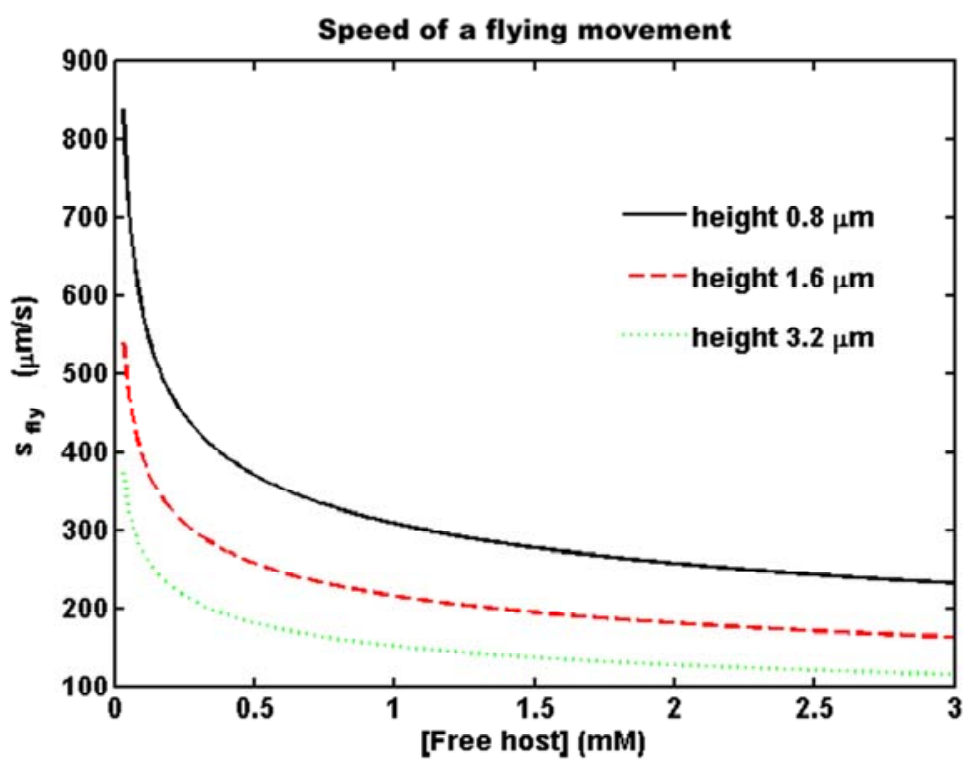

Figure 6.16 Estimated speed for a "fly" movement.

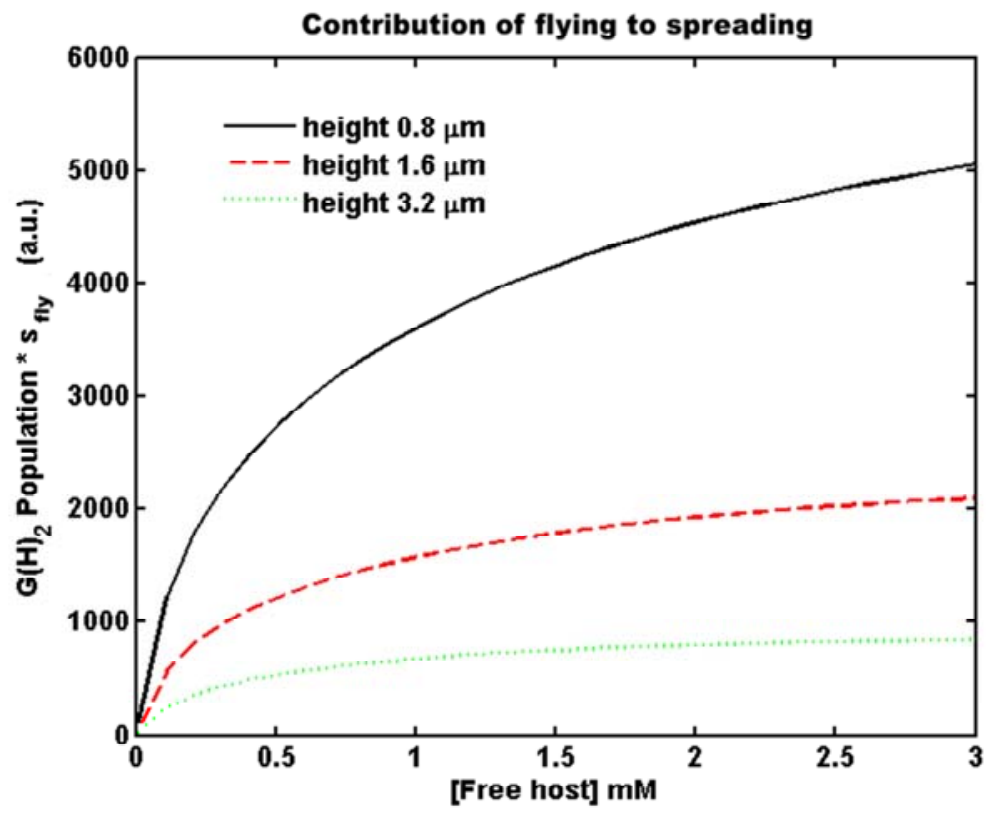

Figure 6.17 Estimated contribution of flying mode to the spreading process. 
A plot of $T_{1 / 2}$ vs the free host concentration (Figure 6.14) shows that the residence time increases with the host concentration as well as with the volume height.

The distance $d_{T 1 / 2}$ traveled by the $\mathrm{G}(\mathrm{H})_{2}$ complex in this residence time will be:

$d_{T_{1 / 2}}=\sqrt{4 \mathrm{DT}_{1 / 2}}$

This travel distance (Figure 6.15) shows the same trend, increasing with both free host concentration and solution height. From the two previous quantities, the average speed per "fly" movement can be calculated.

$S_{f l y}=\frac{d_{T_{1 / 2}}}{T_{1 / 2}}$

Although the traveled distance increased with the free host concentration and solution height, the time each flying complex spends "trapped" in the bulk of the solution compensates this. Increasing the solution height makes this more apparent, as shown in Figure 6.14.

Finally, we can estimate the total contribution of "flying" to the spreading process multiplying this speed by the equilibrium population of $G(H)_{2}$, The results are plotted in Figure 6.17, were the dependence of this contribution with the solution height is clear. The shape of the curves resembles the experimental data for free host concentration in the range of 2-8 $\mathrm{mM}$ (Figure 6.1). There is no indication of a local maximum around $1 \mathrm{mM}$, which confirms that flying mechanism alone is not enough to explain the experimental findings.

\subsection{Appendix C: Estimation of "hop" length versus free host concentration}

The time lapse a newly dissociated from the surface $\mathrm{GH}$ complex spends free before binding back to the host-covered surface is defined as $\tau_{\text {hop }}$. The main factor limiting this time is the possibility of complexing another free host from solution, forming $\mathrm{G}(\mathrm{H})_{2}$. This latter complex is being unable to bind back to the surface for an extended period of time, and thus it follows a flying mechanism instead of a hop. This leads to an inequality relating $\tau_{\text {hop }}$ and the free host concentration $[\mathrm{H}]$ : 


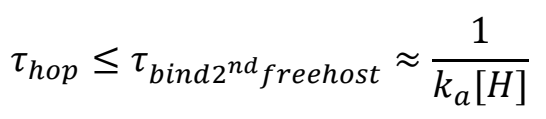

The "hop" length can be estimated with the distance diffused during this time:

$$
d_{\text {hop }} \leq \sqrt{\frac{4 \mathrm{D}}{k_{a}[H]}}
$$

A plot of this equation for the equality condition can be seen in Figure 6.11C, showing a good agreement with the simulations.

\subsection{Acknowledgements}

Dr. András Perl performed the experiments that motivated this chapter and is thanked for his suggestions. Dr. Joost van Opheusden is thanked for fruitful discussions about the model.

\subsection{References and notes}

1. (a) Ehrlich, P. H., J. Theor. Biol. 1979, 81, 123-7; (b) Scobie, H. M.; Young, J. A., Curr. Opin. Microbiol. 2005, 8, 106-12.

2. Mulder, A.; Huskens, J.; Reinhoudt, D. N., Org. Biomol. Chem. 2004, 2, 3409-24.

3. (a) Lund, K.; Manzo, A. J.; Dabby, N.; Michelotti, N.; Johnson-Buck, A.; Nangreave, J.; Taylor, S.; Pei, R.; Stojanovic, M. N.; Walter, N. G.; Winfree, E.; Yan, H., Nature 2010, 465, 206-10; (b) Gu, H.; Chao, J.; Xiao, S.-J.; Seeman, N. C., Nature 2010, 465, 202-5; (c) Liao, X.; Petty, R. T.; Mrksich, M., Angew. Chem. Int. Ed. 2010, 123, 732-4.

4. (a) Yang, J.; Monine, M. I.; Faeder, J. R.; Hlavacek, W. S., Phys. Rev. E Stat. Nonlin. Soft Matter Phys. 2008, 78, 031910; (b) Monine, M. I.; Posner, R. G.; Savage, P. B.; Faeder, J. R.; Hlavacek, W. S., Biophys. J. 2010, 98, 48-56.

5. (a) Ludden, M. J. W.; Reinhoudt, D. N.; Huskens, J., Chem. Soc. Rev. 2006, 35, 1122-34; (b) Huskens, J., Curr. Opin. Chem. Biol. 2006, 10, 537-43.

6. Mulder, A.; Onclin, S.; Peter, M.; Hoogenboom, J. P.; Beijleveld, H.; ter Maat, J.; Garcia-Parajo, M. F.; Ravoo, B. J.; Huskens, J.; van Hulst, N. F.; Reinhoudt, D. N., Small 2005, 1, 242-53. 
7. (a) Ludden, M. J. W.; Mulder, A.; Tampe, R.; Reinhoudt, D. N.; Huskens, J., Angew. Chem. Int. Ed. 2007, 46, 4104-7; (b) Ludden, M. J. W.; Li, X.; Greve, J.; van Amerongen, A.; Escalante, M.; Subramaniam, V.; Reinhoudt, D. N.; Huskens, J., J. Am. Chem. Soc. 2008, 130, 6964-73.

8. Nijhuis, C. A.; Huskens, J.; Reinhoudt, D. N., J. Am. Chem. Soc. 2004, 126, 12266-7.

9. Ling, X. Y.; Phang, I. Y.; Reinhoudt, D. N.; Vancso, G. J.; Huskens, J., Int. J. Mol. Sci. 2008, 9, 486-97.

10. Perl, A.; Gomez-Casado, A.; Dam, H. H.; Thompson, D.; Jonkheijm, P.; Reinhoudt, D. N.; Huskens, J., Nat. Chem. 2011, 3, 317-22.

11. The word reaction will be used here to describe the association and dissociation of supramolecular complexes, not any modification in the covalent chemical bonds of the molecules.

12. Gillespie, D. T., J. Phys. Chem. 1977, 81, 2340-61.

13. (a) Laurenzi, I. J.; Diamond, S. L., Biophys J 1999, 77, 1733-46; (b) Hemberg, M.; Yaliraki, S. N.; Barahona, M., Biophys J 2006, 90, 3029-42.

14. Bernstein, D., Phys. Rev. E Stat. Nonlin. Soft Matter Phys. 2005, 71.

15. Mulder, A.; Auletta, T.; Sartori, A.; Del Ciotto, S.; Casnati, A.; Ungaro, R.; Huskens, J.; Reinhoudt, D. N., J. Am. Chem. Soc. 2004, 126, 6627-36.

16. Fernandes, S.; Cabeça, L.; Marsaioli, A.; de Paula, E., J. Incl. Phenom. Macrocyclic Chem. 2007, 57, 395-401.

17. Huskens, J.; Mulder, A.; Auletta, T.; Nijhuis, C. A.; Ludden, M. J.; Reinhoudt, D. N., J. Am. Chem. Soc. 2004, 126, 6784-97.

18. van Rossum, G. Python Reference Manual; Centrum voor Wiskunde en Informatica (CWI): Amsterdam, 1995.

19. Abramoff, M. D.; Magelhaes, P. J.; Ram, S. J., Biophot. Int. 2004, 11, 3642.

20. Novo, M.; Granadero, D.; Bordello, J.; Al-Soufi, W., J. Incl. Phenom. Macrocycl. Chem. 2010, 11, 173-88. 


\section{Summary}

The aim of the work described in this thesis is to study the role of multivalency in the dynamic behavior of multivalent supramolecular systems. Several supramolecular systems have been employed, ranging from $\pi-\pi$ charge-transfer complexes to cucurbit[n]uril $(C B[n])$ and $\beta$-cyclodextrin $(\beta C D)$ host-guest complexes. All these systems have been used before as building blocks to fabricate nanostructures. Understanding their kinetics potentially allows the design of more stable or fast-responding devices.

In Chapter 2, an overview of the concept of multivalency has been given, including a detailed discussion on the contributions of cooperativity to the stability of a multivalent assembly. The concepts of allosteric, chelate and interannular cooperativity were introduced. Special attention was given to the kinetics of multivalent complexes and the use of dynamic force spectroscopy (DFS) to study them.

Chapter 3 described DFS experiments performed in order to measure the kinetics of two different charge-transfer (CT) complexes. Experiments were conducted using a double-tether approach in order to probe the complexes in a solution-like state. In both CT complexes the acceptor molecule was methylviologen (mv), while the donor molecules were naphthol (nph) and pyrene (pyr). The dissociation rate constant of the pyr-mv complex $\left(2 \mathrm{~s}^{-1}\right)$ was lower than the one of the nph-mv complex $\left(14 \mathrm{~s}^{-1}\right)$, which is in agreement with literature reporting a higher equilibrium constant for the pyr-mv complex. Moreover, the difference in the width of the potential wells $(0.4 \mathrm{~nm}$ and 0.16 $\mathrm{nm}$ for the pyr-mv and nph-mv complexes, respectively) correlates with the bigger size of pyr compared to nph. The design of new drugs based on chargetransfer interactions can be assisted by the knowledge of their dissociation kinetics.

In Chapter 4 the binding properties of $\mathrm{CB}[7]$ monolayers on gold were explored by DFS using an adamantyl (Ad) guest. The coverage of these monolayers was found to be imperfect, which is in agreement with literature, and the impact of unspecific binding of the Ad guest to the exposed gold areas was assessed by comparing its dissociation rate constant $\left(0.3 \mathrm{~s}^{-1}\right)$ with the one of the specific $\mathrm{CB}[7]-\mathrm{Ad}$ interaction $\left(0.03 \mathrm{~s}^{-1}\right)$. The ratio between specific and unspecific 
binding (approx. 40:60) is in agreement with the previously reported coverage using electrochemistry. The relatively higher stability of the specific interaction confirms the usefulness of this type of monolayers despite their incomplete coverage.

In Chapter 5 the effects of multivalency on the dissociation kinetics of a hostguest model system were assessed. The binding of mono-, di- and trivalent Ad guests to $\beta C D$ monolayers was studied at the single-molecule level using DFS. In constrast to the case of the $\mathrm{CB}[7]$ monolayers presented in Chapter $4, \beta C D$ monolayers on gold are well-packed, which minimizes the chance of observing unspecific adhesion of the Ad moieties to the supporting gold substrate. Furthermore, the design of the molecular structure of the multivalent guests enabled the use of a theoretical model to predict the dissociation kinetics of such complexes. The monovalent interaction was probed under thermodynamic equilibrium, while higher valency interactions were probed out-of-equilibrium. The ruptures of partially bound di- and trivalent guests were also identified, and these compared well to the ruptures of assemblies of equivalent valency. The theoretical and experimental values of the dissociation rate constants for di- and trivalent $A d-\beta C D$ complexes were in agreement, confirming the absence of allosteric cooperativity in the assembly. Thus, the increases in stability with the number of bonds were interpreted as chelate and interannular cooperativity effects and explained from the distances between the guest moieties in the multivalent guests. The results described in this chapter validate the kinetic model for multivalent interactions, which is key to the design of structures with predetermined stability.

In Chapter 6 a reduced-scale model of a $\beta C D$ monolayer partially covered with divalent Ad guests was described. The evolution in time of a non-homogeneous coverage of fluorescently labeled guests over these $\beta C D$ monolayers, and the dependence of the spreading rate with the concentration of competing free host $(B C D)$ dissolved in a liquid volume covering these monolayers have been studied experimentally before, finding a marked decrease of the spreading rate of divalent guests when the free host concentration was approx. $1 \mathrm{mM}$. The mechanisms responsible for this behavior were addressed by performing Monte Carlo simulations in the reduced-scale model. The results of these simulations supported a molecular picture of the system where a transition from a local spreading process to an in-bulk spreading process occurred when the concentration of free host in solution was increased from about 0.7 to 1.5 $\mathrm{mM}$, which is in agreement with experimental findings. The impact of 
multivalency on the motion of molecules over a surface can play a fundamental role in the understanding of processes occurring at cell membranes and in the development of new molecular devices.

The results discussed in this thesis present a dynamic perspective of several supramolecular interactions. Knowledge of the kinetics of non-covalent systems is of paramount importance to aid the understanding of many biological processes and the design of new devices. In most cases, multiple interactions are established in parallel, following a multivalent strategy. Thus, multivalent kinetic models are required to understand and predict the time evolution of these systems. In this thesis a kinetic model for multivalent interactions has been discussed. This model has been experimentally validated by measuring the dissociation kinetics of multivalent complexes, as well as employed in simulations that help to explain the spreading of these complexes over a receptor surface. Systems employing weaker binding complexes with higher valencies than the ones studied here could show even more interesting dynamic properties. A better understanding of the key cooperativity factors affecting the thermodynamic and kinetic properties of multivalent complexes should assist in the design of new ligands and molecular constructs, which could be applied in developing novel biomedical and nanoscale devices. 



\section{Samenvatting}

Het in dit proefschrift beschreven onderzoek is gericht op de invloed van multivalentie op het dynamische gedrag van multivalente supramoleculaire systemen. Verschillende supramoleculaire systemen zijn onderzocht, variërend van $\pi-\pi$ ladingsoverdrachtscomplexen tot cucurbit[n]uril- (CB[n]) en $\beta$ cyclodextrine- $(B C D)$ gastheer-gast-complexen. Al deze systemen zijn voorheen gebruikt als bouwstenen voor de fabricage van nanostructuren. Begrip van hun kinetiek kan leiden tot het ontwerp van stabielere of snel reagerende systemen.

In hoofdstuk 2 is een overzicht van het concept van multivalentie weergegeven, inclusief een gedetailleerde uiteenzetting over de bijdrage van coöperativiteit aan de stabiliteit van multivalente assemblages. De concepten van allostere, chelaat- en interannulaire coöperativiteit worden geïntroduceerd. Extra aandacht is gegeven aan de kinetiek van multivalente complexen en het gebruik van dynamische krachtspectroscopie (DFS) ter bestudering van deze.

In hoofdstuk 3 worden DFS-experimenten beschreven ter bepaling van de kinetiek van twee verschillende ladingsoverdrachts- (CT) complexen. Experimenten werden met een dubbele linker uitgevoerd, om de complexen in een oplossingsachtige toestand te bestuderen. In beide CT-complexen was methylviologeen (mv) de acceptor, met naphthol (nph) of pyreen (pyr) als donor. De dissociatiesnelheidsconstante van het pyr-mv-complex $\left(2 \mathrm{~s}^{-1}\right)$ was lager dan die van het nph-mv complex $\left(14 \mathrm{~s}^{-1}\right)$, hetgeen in overeenstemming is met de in de literatuur bekende hogere associatieconstante van het pyr-mvcomplex. Daarnaast is het verschil in de breedte van de potentiaalput $(0.4 \mathrm{~nm}$ en $0.16 \mathrm{~nm}$ voor respectievelijk het pyr-mv en het nph-mv complex) in overeenstemming met de grotere afmeting van pyr in verhouding tot nph. Het ontwerp van nieuwe geneesmiddelen op basis van CT-interacties kan ondersteund worden door de kennis van de dissociatiekinetiek.

In hoofdstuk 4 zijn de bindingseigenschappen van monolagen van $\mathrm{CB}$ [7] op goud onderzocht door middel van DFS met adamantaan (Ad) als gast. In overeenstemming met de literatuur is een onvolmaakte bedekking van de monolagen gevonden. De invloed van niet-specifieke binding van de Ad-gast met de onbedekte delen van het goudoppervlak werd bepaald door vergelijking 
van de dissociatiesnelheidsconstante $\left(0.3 \mathrm{~s}^{-1}\right)$ met die van de specifieke $\mathrm{CB}$ [7]Ad-interactie $\left(0.03 \mathrm{~s}^{-1}\right)$. De verhouding tussen specifieke en niet-specifieke binding (circa 40:60) is in overeenstemming met de eerder gerapporteerde bedekkingsgraad, vastgesteld met behulp van elektrochemie. De relatief hogere stabiliteit van de specifieke interactie bevestigt de bruikbaarheid van dit type monolagen ondanks hun onvolledige bedekking.

In hoofdstuk 5 zijn de effecten van multivalentie op de dissociatiekinetiek van een gastheer-gast-modelsysteem bepaald. De binding van mono-, di- en trivalente Ad-gasten op $\beta C D$-monolagen werd door middel van DFS op het niveau van enkelvoudige moleculen onderzocht. In tegenstelling tot de $\mathrm{CB}$ [7]monolagen gepresenteerd in hoofdstuk 4 , vormt $\beta C D$ goed geordende monolagen op goud, die de kans op niet-specifieke adhesie van de Ad-gasten met het onderliggende goudsubstraat minimaliseren. Verder staat het ontwerp van de moleculaire structuur van de multivalente gasten het gebruik van een theoretisch model ter voorspelling van de dissociatiekinetiek van zulke complexen toe. De monovalente interactie werd onder thermodynamisch evenwicht bepaald, terwijl de hoger-valente interacties uit evenwicht bepaald werden. De dissociaties van deels gebonden di- en trivalente gasten werden ook geïdentificeerd, en nauwkeurig vergeleken met dissociaties van assemblages met gelijke valentie. De theoretische en experimentele waardes van de dissociatiesnelheidsconstanten van de di- en trivalente Ad-BCDcomplexen komen overeen, hetgeen de afwezigheid van allostere coöperativiteit in de assemblage bevestigt. Concluderend is de hogere stabiliteit bij een toenemend aantal bindingen geïnterpreteerd als het optreden van chelaat- en interannulaire coöperativiteitseffecten afhankelijk van de afstand tussen de gasteenheden in de multivalente gasten. De resultaten beschreven in dit hoofdstuk bevestigen de toepasbaarheid van het kinetische model voor multivalente interacties, dat de sleutel is voor het ontwerpen van structuren met vooraf bepaalde stabiliteit.

Hoofdstuk 6 beschrijft een gereduceerd schaalmodel van een $\beta C D$-monolaag deels bedekt met divalente Ad-gasten. De verandering in de tijd van een niethomogene bedekking van fluorescent gemarkeerde gasten over deze $\beta C D$ monolagen en de afhankelijkheid van de spreidingssnelheid als functie van de concentratie van concurrerende vrije $\beta C D$ opgelost in een vloeistofvolume op deze monolagen werden reeds eerder experimenteel onderzocht, met als resultaat een duidelijke afname van de spreidingssnelheid van divalente gasten bij een vrije gastheerconcentratie van circa $1 \mathrm{mM}$. De mechanismen 
verantwoordelijk voor dit gedrag werden onderzocht door uitvoering van Monte-Carlo-simulaties in het gereduceerde schaalmodel. De resultaten van deze simulaties ondersteunen het moleculaire plaatje van een systeem waarin zich een overgang van een lokaal spreidingsproces naar een bulkspreidingsproces voordoet als de concentratie van de vrije gastheer in oplossing wordt verhoogd van ongeveer 0.7 tot $1.5 \mathrm{mM}$, wat overeenkomt met de experimentele gegevens. De invloed van multivalentie op de beweging van moleculen over een oppervlak kan een fundamentele rol spelen in het begrip van processen die plaatsvinden in celmembranen en in de ontwikkeling van nieuwe moleculaire toepassingen.

De resultaten in dit proefschrift presenteren een dynamisch perspectief op enkele supramoleculaire interacties. Kennis van de kinetiek van niet-covalente systemen is van groot belang als hulpmiddel in het begrijpen van vele biologische processen en het ontwerpen van nieuwe toepassingen. In de meeste gevallen worden veelvuldige interacties parallel gevormd, volgens een multivalente strategie. Multivalente kinetiekmodellen zijn dus nodig voor het begrijpen en voorspellen van de verandering van deze systemen in de tijd. In dit proefschrift is een kinetisch model voor multivalente interacties beschreven. Dit model is experimenteel bevestigd door het meten van de dissociatiekinetiek van multivalente complexen en gebruikt in simulaties die de spreiding van deze complexen over een receptoroppervlak helpen verklaren. Systemen van zwakkere bindingscomplexen met een hogere valentie dan die hier onderzocht zijn, zouden zelfs meer interessante dynamische eigenschappen kunnen vertonen. Een beter begrip van de belangrijkste coöperativiteitsfactoren die de thermodynamische en kinetische eigenschappen van multivalente complexen beïnvloeden, zou kunnen helpen bij het ontwerpen van nieuwe liganden en moleculaire constructies, die gebruikt zouden kunnen worden bij de ontwikkeling van nieuwe biomedische toepassingen en applicaties op de nanoschaal. 



\section{List of publications}

- Probing multivalent interactions in a synthetic host-guest complex by dynamic force spectroscopy.

A. Gomez-Casado, H. H. Dam, M. D. Yilmaz, D. Florea, P. Jonkheijm and J. Huskens

Journal of the American Chemical Society 2011 (in press)

- Interlaboratory round robin on cantilever calibration for AFM force spectroscopy.

J. te Riet, A. J. Katan, C. Rankl, S. W. Stahl, A. M. van Buul, I. Y. Phang, A. Gomez-Casado, J. W. Gerritsen, A. Cambi, A. E. Rowan, G. J. Vancso, P. Jonkheijm, J. Huskens, T. H. Oosterkamp, H. Gaub, P. Hinterdorfer, C. G. Figdor and S. Speller Ultramicroscopy 2011 (in press)

- Gradient-driven motion of multivalent ligand molecules along a surface functionalized with multiple receptors.

A. Perl, A. Gomez-Casado, D. Thompson, H. H. Dam, P. Jonkheijm, D. N. Reinhoudt and J. Huskens

Nature Chemistry 2011, 3, 317-22

- Enhanced propagation length for surface plasmon polaritons on a buried metal grating .

J. Jose, F. B. Segerink, J. P. Korterik, A. Gomez-Casado, J. L. Herek and H. L. Offerhaus Journal of Applied Physics 2011, 109, 064906

- Direct patterning of covalent organic monolayers on silicon using nanoimprint lithography.

W. Voorthuijzen, M. D. Yilmaz, A. Gomez-Casado, P. Jonkheijm, W. van der Wiel and J. Huskens

Langmuir 2010, 26, 14210-5

- Topologically matching supramolecular $\mathrm{n} / \mathrm{p}$-heterojunction architectures.

R. Bhosale, A. Perez-Velasco, V. Ravikumar, R. S. K. Kishore, O. Kel, A. Gomez-Casado, P. Jonkheijm, J. Huskens, P. Maroni, M. Borkovec, T. Sawada, E. Vauthey, N. Sakai and S. Matile Angewandte Chemie International Ed. in English 2009, 48, 6461-4 
- Ordered and oriented SHJ surface architectures: completion of the primary color collection.

R. S. K. Kishore, O. Kel, N. Banerji, D. Emery, G. Bollot, J. Mareda, A. Gomez-Casado, P. Jonkheijm, J. Huskens, P. Maroni, M. Borkovec, E. Vauthey, N. Sakai and S. Matile

Journal of the American Chemical Society 2009, 131, 11106-16

- Porous multilayer-coated PDMS stamps for protein printing.

H. Xu, A. Gomez-Casado, Z. Liu, D. Reinhoudt, R. Lammertink and J. Huskens

Langmuir 2009, 25, 13972-7

- Hooke: an open software platform for force spectroscopy.

M. Sandal, F. Benedetti, M. Brucale, A. Gomez-Casado and B. Samori

Bioinformatics 2009, 25, 1428-30

http://code.google.com/p/hooke/

\section{Publications in preparation:}

- Monte Carlo simulations of the spreading of divalent molecules on a receptor surface .

A. Gomez-Casado, A. Perl, J. H. J. van Opheusden, P. Jonkheijm and J. Huskens

- Recognition properties of cucurbit[7]uril self-assembled monolayers studied with force spectroscopy.

A. Gomez-Casado, P. Jonkheijm and J. Huskens 


\section{Acknowledgements}

After more than four years of work, this thesis is finished. I want to thank here to many people who helped me, both scientifically and socially, to accomplish this. Such large amount of people makes this section hard to write, both for the fear of forgetting someone and for the fact that the acknowledgements are traditionally the first (and sometimes only) part of the thesis that will be read, making the impact factor of this section rise like foam.

I will start thanking my supervisors: Jurriaan, thanks for giving me the opportunity to work in your group. Considering my chemical illiteracy at the time of my arrival, your confidence that I would be able to survive in a chemistry lab was indeed greater than my own. On the early months (when it is easier to wander away from any meaningful approach) you kept me on track and were extremely patient with my struggles. A bit later than that Pascal arrived to the group and became my daily supervisor. Pascal, those who never had a meeting with you are missing the unique experience of entering your office with a set of (one thought) not so convincing results and leave it deciding about where to publish them after receiving one of your massive doses of enthusiasm. Thanks to both of you for your helpful supervision and suggestions during our meetings, a few times convincing me (not an easy task!) to follow this or that approach, many others giving me heaps of freedom that I could enjoy to do things that were not in the plan. Of course, thanks for reading, rereading, re-[n]-reading and correcting manuscripts and the proofs of this thesis, and for the nice advice on how to improve them. I learnt a lot from you during these years.

Research is hard enough when everything works as it should, and for their efforts trying to achieve such utopical state I thank our technicians, Marcel who always greeted me with his friendly "hello sir" while supplying me with dozens of gold substrates and AFM tips and Richard, who had to endure my petitions to install strange software that no one ever used before (and probably nor will be used after) in the group. I want also to thank Clemens for his assistance whenever the Picoforce decided to have a bad day (by the end of my PhD, more often than not). Thanks also to the secretaries, Izabel, Nicole, (and before Gerardine and Danielle), for their help in navigating through the thousand different forms that I had to fill during my PhD. 
During these four years I met many people from outside the group. All of them where invaluable scientific input for me. I enjoyed discussing and collaborating with Naomi Sakai, from the University of Geneva, Jincy Jose from the OS group, Joost te Riet from the Radboud University and Joost van Opheusden from Wageningen University. Thanks to all. The single molecule conference in Linz gave me the opportunity to meet Massimo Sandal, with whom I shared both the interest for open software and the hardships of force spectroscopy. His data analysis tool was the cornerstone that I needed to build up a consistent and (more or less) efficient filtering procedure for the hundredths of thousands of force curves that I collected during my PhD. Thanks to him, to Marco and to Fabrizio for creating Hooke. I will use this opportunity to campaign a bit in favor of open software. Although I had to settle for Microsoft Office when writing manuscripts and this thesis, I managed most part of the daily work using GNU tools. I want to thank the contributors of linux in general, the GNU Scientific Library, Python, GIMP, ImageJ, qtiplot, gnumeric, Scilab, Gwyddion and R, who enabled me to do so much without the need to pay several thousand euros in licenses.

Now the time to focus on the closest colleagues, which are many. MNF is a quite big group, and effectively even bigger if you take in account that (the late) SMCT, (the new) BNT and (if you are involved with AFM, special thanks here to Anika, In Yee, Edit, Joost and Peter Schön) MTP groups share the labs, many meetings and most of the equipment. András, your research gave me the perfect excuse to spend some of my time trying to shave milliseconds from each cycle of the simulations of Chapter 6 , one of the most challenging programs I ever did. The study of multivalent guests would have been totally impossible without the chemical expertise of Deniz, Daniel and Henk. Henkino, apart from my private molecule dealer you were a constant source of fun and I had the pleasure to sit next to you for a few years (unfortunately your fume hood was also a constant source of nasty chemicals and radioactive isotopes, which were no so pleasant).

Trying to create a dust-free island in an organic chemistry lab was a battle that I lost from the start. Several people contributed to undermine my futile attempts to revert entropy inside my fume hood. Elizabetta, Silvia, Mustafa and Pim. Thanks to all for the nice time in the lab, actually you were all very clean (with the exception of a certain metal bath that sprinkled all over the place). 
Huaping, I greatly enjoyed talks about pretty much everything while we waited for the images of your porous stamps. Thanks for the delicious dinners and beers in Beijing. Thanks also to Prof. Xi Zhang, from the TsingHua University, for his hospitality and for the opportunity to visit his group in China. Thanks to all the members of Xi's group, and in particular to Yiheng, Yiliu, Kai and XinXin, who showed me many beautiful places in Beijing and the Great Wall. I also want to thank Prof. Shuxun Cui and his students, from the Soutwest Jiaotong University, for their hospitality while I visited Chengdu. Thanks to a current member of MNF, Angel, who rescued me from boredom a couple times while in Beijing for the modest price of information about life in the Netherlands.

I am happy to have shared not only a lab but also a roof (including its many leakages) with very interesting people that I thank for their friendship. Martine, effectively the mum of Javastraat, merci beaucoup for showing me very nice new recipes, for the evenings you often organized and for your interest in learning Spanish, which provided hilarious moments. Victoria, you worked so long hours that I considered subletting your room. Buena suerte con el peque, keep the chips out of his reach (and out of the washing machine too). Arancha, you also contested for the title of the person living the least hours/day in the house, sadly, our country did not know how to appreciate a brilliant scientist as you are, and now I hear you are "Breaking Bad" and joining organized crime (a.k.a. politics). Carmen, your mid-afternoon siestas followed by preparing a mega tea-pot and studying at dinner time never ceased to impress me. Francesca S., your stay was brief but pleasant, although the living room chairs don't agree with me here. Eddie, your suit is still in the little room (but the little room is not there anymore, ups). And Ralu, you are the nucleating particle of groups of friends wherever you go, which should be a good measure of what a nice person you are. Thank you for so many game evenings, for your help painting walls and your moral support when the paper collapsed immediately after, and for being my paraninf. It's a pity that such nice social place as Javastraat was finally lost, but I am sure it was not the fault of the two last people from the group that occupied it, Carlo, probably the calmest Italian man I ever met, and little Shu Han. Shu Han (HsuÚ!), your candor was as funny as it could be dangerous, thank you for countless funny, serious, happy, sad, scientific and sportive moments.

The Spanish speaking community in the group was a fluctuating set that sometimes menaced to take control of the lab and others was reduced to barely a couple of people. Thanks to the old-timers that I met at my arrival, 
Fernando and Lourdes, Olga and Alessio, Mirko and Itxaro (thanks for hosting me in Madrid), Miguel Angel and Marina, (so many couples, I see a pattern here), Laura and Yanina. Las panas, Maryana and Jealemy, were there almost all the time and I had to get used to things, sorry, vainas, being chévere, and happening ahorita. Jealemy, ya queda poco, corre! Although he arrived here wearing a serious guy aura, Nachete contributed to many of the most hilarious moments of my PhD, gracias. I shared some short but nice time with two Erasmus students, Miguelito and Alfonso. Finally, moltes gràcies to the (not so fresh anymore) freshman, Jordi (seguro que estabas encuriosido a ver si te ponía en este apartado o no) for many nice moments at Javastraat, at Populierstraat and in the workweek to UK.

Talking about that workweek I thank my roommates of that travel. Albert, which is the one and only chemist I know capable of delivering a presentation completely filled of synthesis and yet keep me interested. Kim, you and I have shared rooms in half of Europe (Portugal, Spain, Denmark, UK, unfortunately not Turkey). Although we could not be more different in some aspects I always enjoyed your company and nearly all the times (that is, when I was able to decipher it) your conversation and tongue-in-cheek remarks. Sven, how could I not like your comments, almost as tactful as mine. And Pieter, not only the master of fire in every barbeque, but a great friend that you can always count on, dankjewel for so many nice dinners, party nights, urgent calls, scary negotiations with unwelcoming locals and for all the help organizing the day of my defense.

Thanks to all the people that were not mentioned yet: Francesca C.(oNstantini) and Riccardo the brilliant, Riccardo popeye, Alessandro, Emanuela, Emanuele, Roberto (not Riccardo!), Moira and il grande capo, Aldrik, closing the Italian battalion, and to Mudassir, Tom, Bianca, Jasper, Rajesh, Nicolai, Raquel, Tieme, Anna, Christian, Dennis, Oktay, Bartek, Soco, XingYi, Duan, Vijay, Martijn, Erhan, Anne, Sachin, Joanna, Dodo, Rick and Rik, DaeJune, Ula, Asha, Lanti, Veera, Janet, Yiping, ChienChing, Peter, Sarah, Yujie and Bala, for all the discussions, the help when I had to find some compound, and the fun at sportdays, borrels, sinterklasses...

I also must thank the friends I left back in Spain, which I see not as much as I would like, but every holiday helped me to recharge my batteries and temporarily disconnect from research, los físicos, especialmente Homer, Indi, 
Chema, Mónica, Helena and Berna, los "itis", the kamikaze midday-soccerplayers, and of course my oldest friends, Ramón, Isa, Rubén and Ángel.

Finally, thanks to my parents, that gifted me the education that made possible for me to be here, (Papá, mamá, gracias por darme una formación, fomentar mi curiosidad desde pequeño y hacer de mí un devorador de libros, sin vuestro esfuerzo y paciencia yo no habría podido ni soñar con empezar un doctorado). Good luck to my sister who is herself in the process of becoming the other type of doctor. And thanks to Melanie, you shared with me all the good and bad moments of the second half of my PhD, just having you close made the good ones much better and the bad ones not that important. 



\section{About the author}

Alberto Gomez-Casado was born on the $27^{\text {th }}$ of October in 1981, in Salamanca, Spain. He studied Physics and Computer Engineering at the University of Salamanca. After graduating in Physics in $\mathbf{2 0 0 5}$ he obtained his Master degree on Biophysics at the Universidad Autónoma de Madrid, where he studied the adhesion of bacteria on surfaces presenting different chemical functionalities under the supervision of Prof. Marisela Vélez and Dr. Miguel Manso. In December 2006 he started his PhD research working at the group of Prof. Jurriaan Huskens, at the University of Twente. The results of this research are presented in this thesis. 